\section{The Design and Construction of the Muon Arm in PHENIX}

\author{
Progress Report for the period
}

December 15, 1994 - December 1, 1995

Grant DE-FG05-88ER-40445

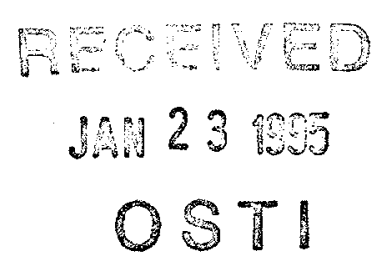

Submitted by

The Intermediate Energy Nuclear Physics Group

Department of Physics and Astronomy

Louisiana State University

and Agricultural and Mechanical College

Baton Rouge, LA 70803-4001

P. N. Kirk

Principal Investigator

December 15, 1995

Prepared for the Division of High Energy and Nuclear Physics

United States Department of Energy
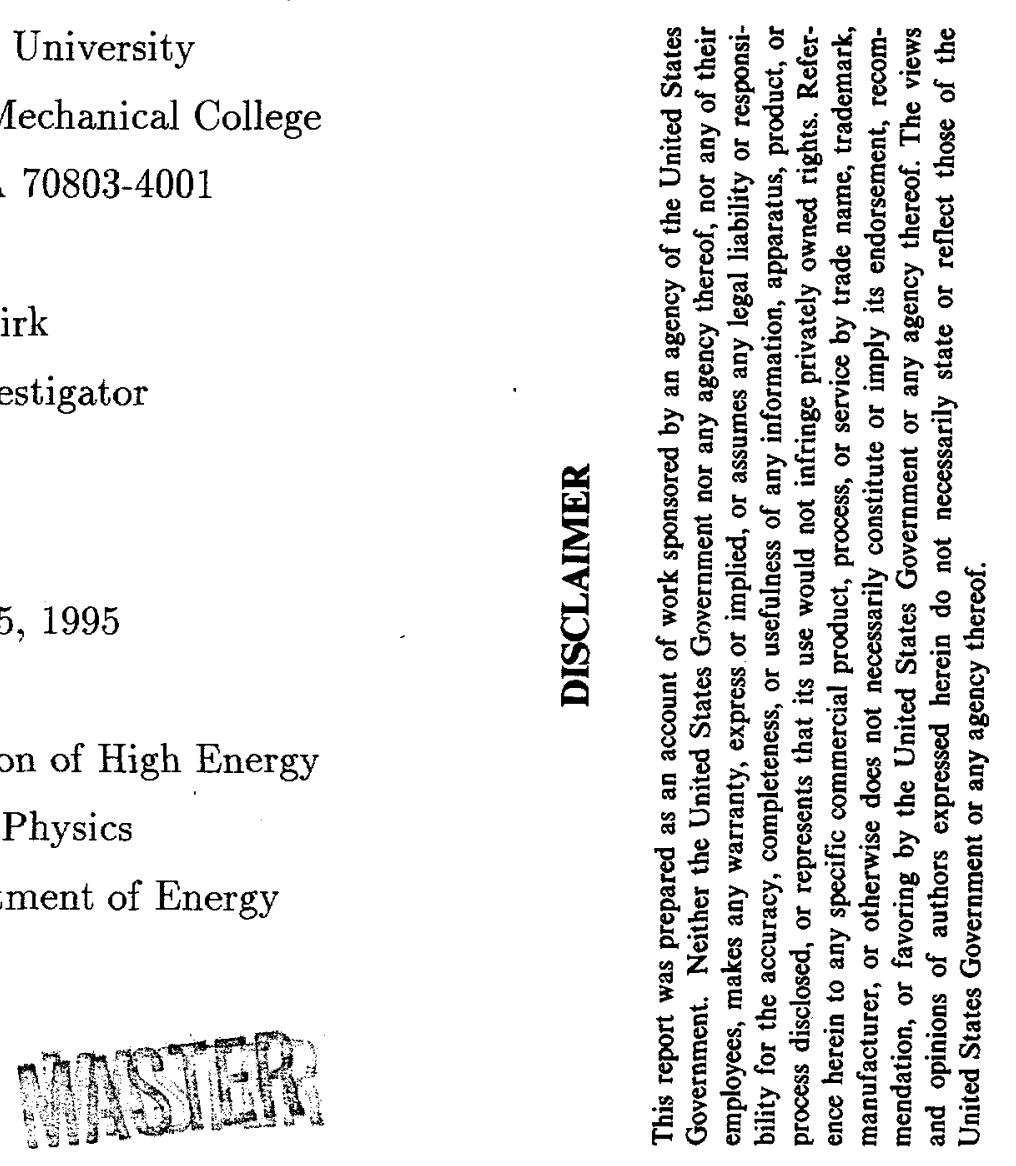

DISTRIBUTION OF THIS DOCUMENT IS UNLIMTED 


\section{DISCLA IMER}

Portions of this document may be illegible in electronic image products. Images are produced from the best available original document. 


\section{Introduction}

The purpose of this report is to describe activities performed by the Intermediate Energy Nuclear Physics Group (IENPG) at the Louisiana State University (LSU) under the terms of grant DE-FG05-88ER40445. The report will cover the period of time between approximately December 15, 1994, and the present.

During the preceding year we have been actively involved with three collaborations - the PHENIX Collaboration, the DLS Collaboration, and the E866 Collaboration at Fermilab that are the foci of our activities. In addition we are members of a fourth collaboration, the AMY Collaboration at KEK, and in concert with other members of that collaboration we are working diligently to terminate all its ongoing activities.

\section{Publications}

During the calendar year 1995 four papers that were supported in part by grant DE-FG0588ERO40445 appeared in print and a fifth was officially accepted for publication. They are:

1. A high- $\mathrm{Q}^{2}$ measurement of the photon structure function $\mathrm{F}_{2}^{\gamma}, \mathrm{S}$. K. Sahu et al., Phys. Lett. B 346, (1995) 208

2. A determination of $\alpha_{s}$ in $e^{+} e^{-}$annihilation at $\sqrt{s}=57.3 \mathrm{GeV}, \mathrm{Y}$. K. Li et al., Phys. Lett. B 355, (1995) 394

3. A measurement of Bose-Einstein correlations in $e^{+} e^{-}$annihilation at TRISTAN, S. K. Choi et al., Phys. Lett. B 355, (1995) 406

4. Study of two particle azimuthal correlations in $e^{+} e^{-}$annihilation at $\sqrt{s}=58 \mathrm{GeV}$, S. Kanda et al., Phys. Rev. D,52, (1995) 4872

5. Measurement of charm production in two photon processes using lepton events at TRISTAN, T. Aso et al., Phys. Lett. B, (in press)

\section{Personnel}

Three of the members of the IENPG, Zhi-fu Wang, Ying-chao Wang, and P. N. Kirk, should require no introduction to the reader of this report, but in May, 1995, our numbers increased through the addition of two undergraduates, Mr. James Gregurich and Mr. Micah McDaniel. These two young men have been extensively involved with the calculation of the resolution function for the DLS and have contributed in significant ways to the contents of this report, and it is with pleasure that we introduce them to the DOE at this time.

Mr. Gregurich In 1989 he graduated from 
Opelousas Senior High School where he was the recipient of many honors. He was designated a "Louisiana Scholar" by the Louisiana State Board of Elementary and Secondary Education. He was also a member of the National Honor Society, and he was selected to receive the "Academic Fitness Award" by George H. W. Bush, a prominent politician at the time. Mr. Gregurich entered the Louisiana State University in Eunice, Louisiana, (LSUE) in 1988 under the auspices of the early admissions program. He received an associates' degree from LSUE in 1991 and entered the Louisiana State University in Baton Rouge immediately thereafter as a candidate for the B.S. degree in physics. Mr. Gregurich plans to begin graduate studies in physics in January, 1997, and hopes to enroll at LSU.

Mr. McDaniel is perhaps the most intellectually gifted student we have seen in years. He and graduated from St. Amant High School in 1991. His academic potential being rather obvious, he was awarded both an Alumni Association Scholarship and a Peagues engineering scholarship upon entry to LSU in August, 1991. During his sophomore year he was elected the outstanding sophomore in physics. More recently he was awarded the locally prestigious Pasqualli Porcelli scholarship in mathematics. He will graduate in May, 1996, having earned degrees simultaneously in physics, electrical engineering, and computer engineering. He hopes to enroll in graduate school at Cornell University in the fall of 1996.

\title{
Activities of the IENPG
}

\author{
The PHENIX Collaboration
}

During the past year we began simulating the interactions between Au projectiles of full energy with residual gas in the beampipe at RHIC. We delivered a seminar on the subject in July of this year at BNL and spoke once again on the simulation of beam-gas interactions at a recent meeting of the muon subgroup in Kyoto, Japan. In this report we would like to summarize briefly the motivation for this simulation and its current status.

The author has learned much from numerous conversations with Sebastian White and Kimo Welch at BNL, and it is a pleasure to acknowledge their tutelage concerning the vacuum and the configuration of the beamline. In addition Sue Norton at BNL spent many hours locating the blueprints that were indispensable to the simulation of the beamline.

Based upon conversations with White and Welch, we assumed the total pressure inside the warm sections of the beampipe to be $5 \times 10^{-10}$ Torr and the composition of the residual gas to be $90 \%$ hydrogen, $5 \%$ carbon monoxide, and $5 \% \mathrm{CH}_{4}$. Similarly, we assumed the total pressure inside the gas in the cold sections of the beampipe to be $1 \times 10^{-11}$ Torr and the composition of the gas to be $95 \%$ hydrogen and $5 \%$ helium.

Throughout the simulation we have assumed that the heavy nuclei carbon and oxygen could be regarded as nitrogen. The primary justification for this assumption was pure expediency; we possessed a HIJET file for nitrogen but none for carbon or oxygen. In addition the 
chemical properties of the target are irrelevant to the simulation. In fact the identity of the target is significant only insofar as the assumed cross section for interaction is a function of its atomic number. Within the context of our simple geometrical model the total cross section for nitrogen is, to within $\frac{1}{3} \%$, equal to the average of the cross sections for carbon and oxygen. For this reason we are confident that this approximation involves no essential compromise, and we will throughout the remainder of this discussion refer to atoms of carbon and oxygen as "pseudo-nitrogen".

A simple calculation yields the number density for atoms of the various chemical species in the warm and cold bore. One finds that the number density of hydrogen in the warm bore is $3.22 \times 10^{7} \mathrm{~cm}^{-3}$ and that the number density of pseudo-nitrogen in the warm bore is $2.41 \times 10^{6}$ $\mathrm{cm}^{-3}$. The number densities for hydrogen and helium in the cold bore are $4.37 \times 10^{7} \mathrm{~cm}^{-3}$ and $1.15 \times 10^{6} \mathrm{~cm}^{-3}$, respectively.

The total cross sections for interaction between an atom of $\mathrm{Au}$ and a target of atomic number "A" was assumed to be:

$$
\sigma=\pi \times\left(1.2 \times 10^{-13}\right)^{2} \times\left(197^{1 / 3}+A^{1 / 3}\right)^{2} \quad c m^{2} .
$$

The conceptual foundation for the preceding formula is the so-called "black sphere" model in which each nucleus is assumed to be a sphere of radius $1.2 \times A^{1 / 3}$ fermis. The interaction radius is assumed to be the sum of the two nuclear radii, and the total cross section for interaction is the area of a circle with a radius equal to the interaction radius. The cross sections that one calculates on the basis of this model are 2.10 barns, 2.48 barns, and 3.06 barns for hydrogen, helium, and nitrogen, respectively.

If one assumes that each bunch of $\mathrm{Au}$ ions in the accelerator comprises $1 \times 10^{9}$ nuclei and that the bunches are separated in time by 112 nanoseconds, then a simple calculation shows that the interaction rate per meter of warm bore is $67 \mathrm{hz}$ and that the interaction rate per meter of cold bore is $84 \mathrm{hz}$. The total distance between the interaction point and the first significant bend in the beamline is approximately 100 meters, so we shall adopt 100 meters as a meaningful distance for estimating the interaction rate between $\mathrm{Au}$ projectiles and residual gas in the beamline. For simplicity we shall assume that the total length comprises 50 meters of both warm bore and cold bore. Because the interactions may occur on either the north side of the apparatus or the south side, we must multiply by a factor of two. A simple calculation based upon these assumptions shows that the resulting interaction rate is $15 \mathrm{khz}$.

The interactions of interest to the muon subgroup of PHENIX are central collisions between $\mathrm{Au}$ and $\mathrm{Au}$, and in calculating the rate for these interactions we shall follow largely the discussion in chapter 11 of the conceptual design report. We assume a luminosity of $2 \times 10^{27}$ $\mathrm{cm}^{-2} \mathrm{~s}^{-1}$ and that the total cross section for interaction between $\mathrm{Au}$ and $\mathrm{Au}$ is given by the formula presented a few lines above with the atomic number of the target set equal to 197 . 
Finally, we assume that the cross section for central collisions is $10 \%$ of total cross section. Under these assumptions, one calculates a rate of $1.2 \mathrm{khz}$ for central collisions between $\mathrm{Au}$ and $\mathrm{Au}$.

All the assumptions listed in the preceding paragraph are subject to legitimate challenge, and our purpose here is not to proselytize on behalf of these assumptions or any other assumptions that others might propose but rather to establish the motivation for a serious study of interactions between the beam and residual gas in the beampipe. The conclusion to be drawn from the preceding numbers is abundantly clear in our opinion, and it is invariant with respect to reasonable variations in the assumptions: The cross section for interactions between beam and residual gas is approximately one order of magnitude larger than the cross section for central collisions between $\mathrm{Au}$ and $\mathrm{Au}$.

The event rate itself may be alarming, but in the absence of simulation one cannot answer even fundamental questions, such as what fraction of the interactions produce a level-1 trigger in the muon arm? The purpose of our activities during the preceding year has been to establish a quantitative mechanism for answering this and other fundamental questions.

The simulation involves three separate components: a model for the interactions between beam and residual gas; a model for the geometry of the beamline and the tunnel; and an event generator that controls the selection and distribution of events. These three components are added to PISA, in which resides the description of PHENIX, and tracking is carried out by GEANT and its associated subroutines.

In early April, 1995, the muon subgroup met at LSU and selected HIJET as the most useful of the available models for interactions between beam and residual gas. Subsequent to that decision Ron Longacre at BNL, for whose assistance we are most grateful, provided HIJET files for hydrogen, helium, and nitrogen. Each file contains 100 interactions between a $\mathrm{Au}$ projectile of full energy and an atom of the the specified chemical species, and all results to date have been generated on the basis of these HIJET files.

The geometry of the beamline was reconstructed on the basis of blueprints and construction drawings, some of which were laboriously extracted from the archives at BNL. The acquisition of prints was, by far, the most arduous and time-consuming activity of the past year, and it is an activity that would have been impossible - absolutely impossible - without the generous assistance provided by Sue Norton at BNL, to whom we award, in abscentia, the Croix de Guerre.

As of the current time the following magnets have been incorporated into the simulation: D0, DX, Q1, CQ2, CQ3C, CQ4T, CQ5T, D5I, D5O, CQ6T, D6, CQ7, CQ8, D8, CQ9S, and D9. In addition, the concrete shielding that encloses the tunnel between the interaction point and magnet D5 has been included in the simulation. Because the shape of the tunnel does not coincide with one of the standard shapes defined in GEANT, we have approximated the shielding by means of rectangular slabs with dimensions that were chosen in accordance with construction drawing S-5, sheet 28 . 
The event generator is a fortran subroutine. Its specific functions are to open the appropriate HIJET file or files; to calculate the coordinates of each interaction point; to read data from the appropriate HIJET file; to store the data in the proper common block for subsequent tracking by GEANT; and to load a large number of arrays from which the characteristics of the initial ensemble of particles may be calculated. The execution of the event generator is controlled entirely by variables that are set by the user.

The aspects of the simulation for which we have been specifically and solely responsible are the writing and debugging of the geometry package, which is a fortran subroutine entitled rhic_magnet_install.f, and the event generator, which is also a fortran subroutine called beam_gas_evt.f. Because of the substantial length of these routines we have decided to omit them from the body of this report but to include them as appendices "A" and "B". We doubt that the reader of this report will derive any particular spiritual enlightment from a perusal of these routines, and they are not included here for this purpose. Indeed, these routines are out-of-date as of the present moment, and they will be obsolete by the time they reach the hands of the reader. We ask the reader not distribute copies of these routines as superior versions are available upon request directly from the author. Nonetheless the routines are well documented and easy to read, and they should provide the reader with some understanding of the direction of our efforts and the magnitude of the undertaking.

On the following pages are shown two drawings, the first being a view from the top and the second being a view from the side, in which are traced the projections of the magnets onto horizontal and vertical planes. The horizontal and vertical scale factors in these drawings are 0.00045 and 0.012 , respectively, and we call to the reader's attention the resulting gross distortion of angle. These two drawings were produced from within GEANT, and they therefore represent the actual implementation of the magnets and the concrete shielding by our routine rhic_magnet_install.f.

During our simulations we encountered an unanticipated problem with HIJET. It turns out that HIJET treats the nucleons within both projectile and target as if they were free and independent particles. Specifically HIJET includes no provision for coalescence of nucleons into nuclear fragments. For this reason collisions that are modeled by means of HIJET include far too many free nucleons in the final state, and, in addition, the differential distributions with respect to the momentum of the free nucleons stand in startling contrast to experimental measurement.

As graphic illustration of these remarks we show on the following pages the number distributions for protons and neutrons that are produced in collisions between Au projectiles of full energy and hydrogen. Also shown is the differential distribution with respect to momentum for protons. The width of this differential distribution, which results from compounding Fermi momentum with the Lorentz boost from the barycentric system to the laboratory is far too large. 


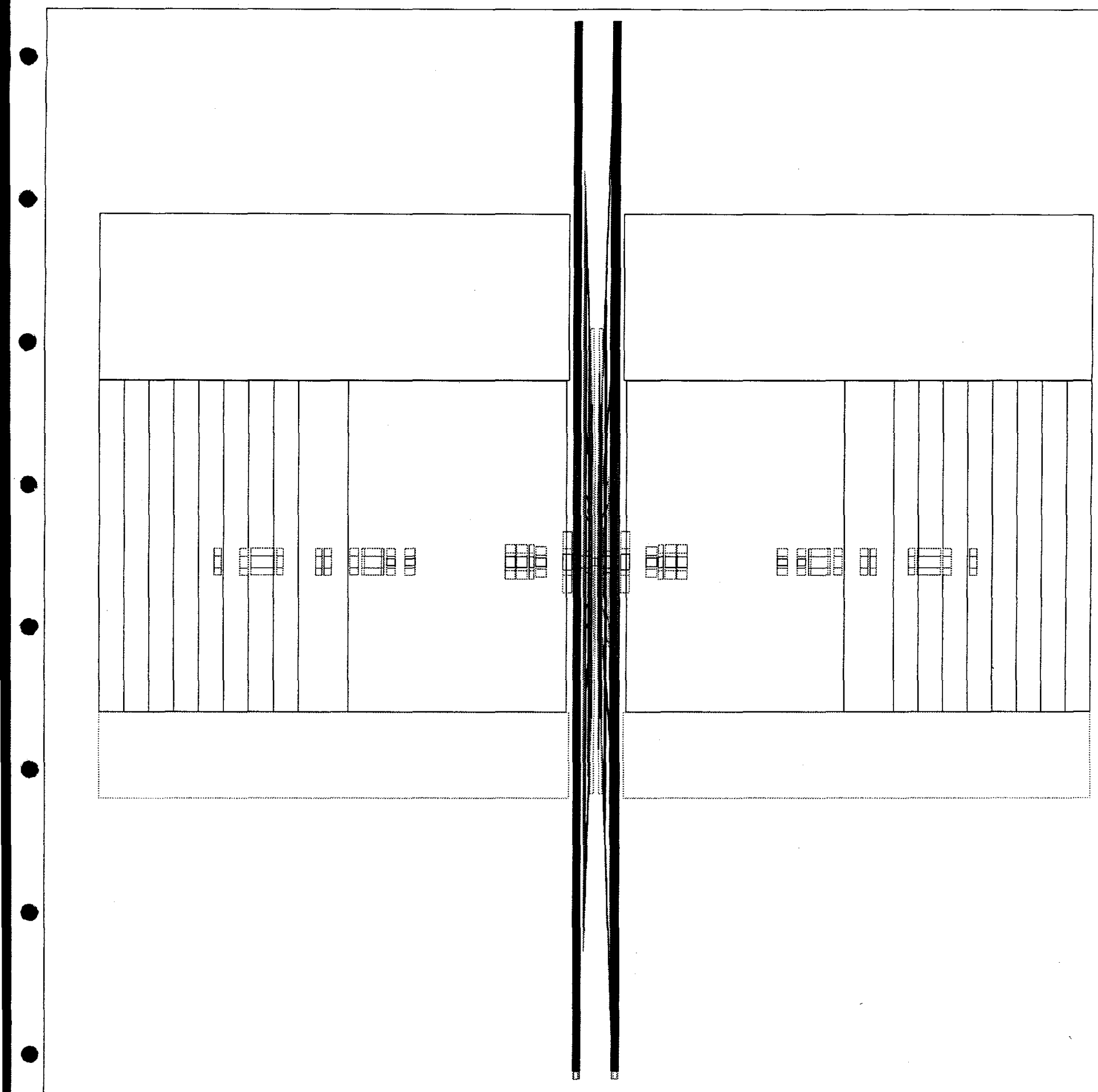

Fig 2 
Number Distributions for Nucleons
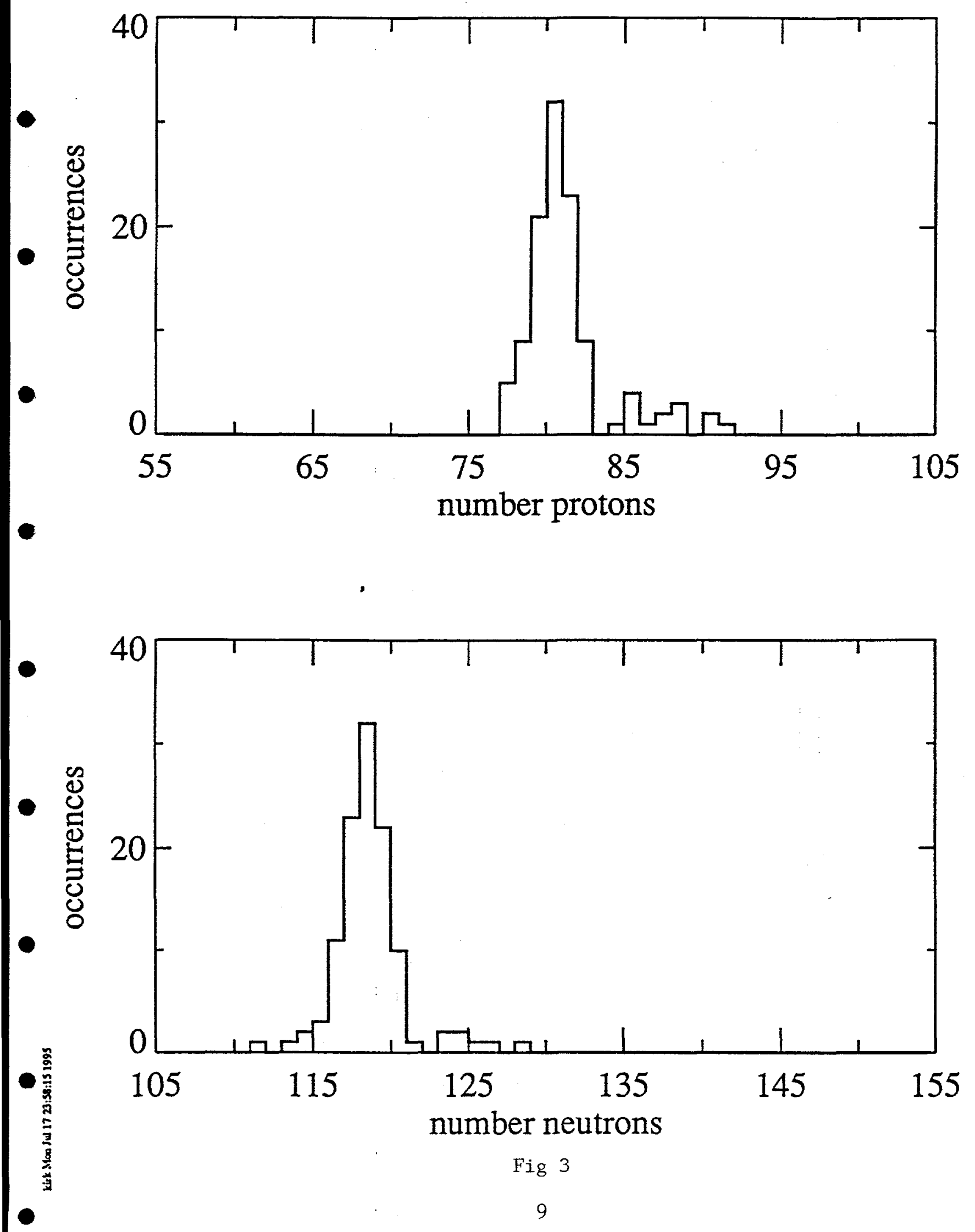
Differential Distributions for protons
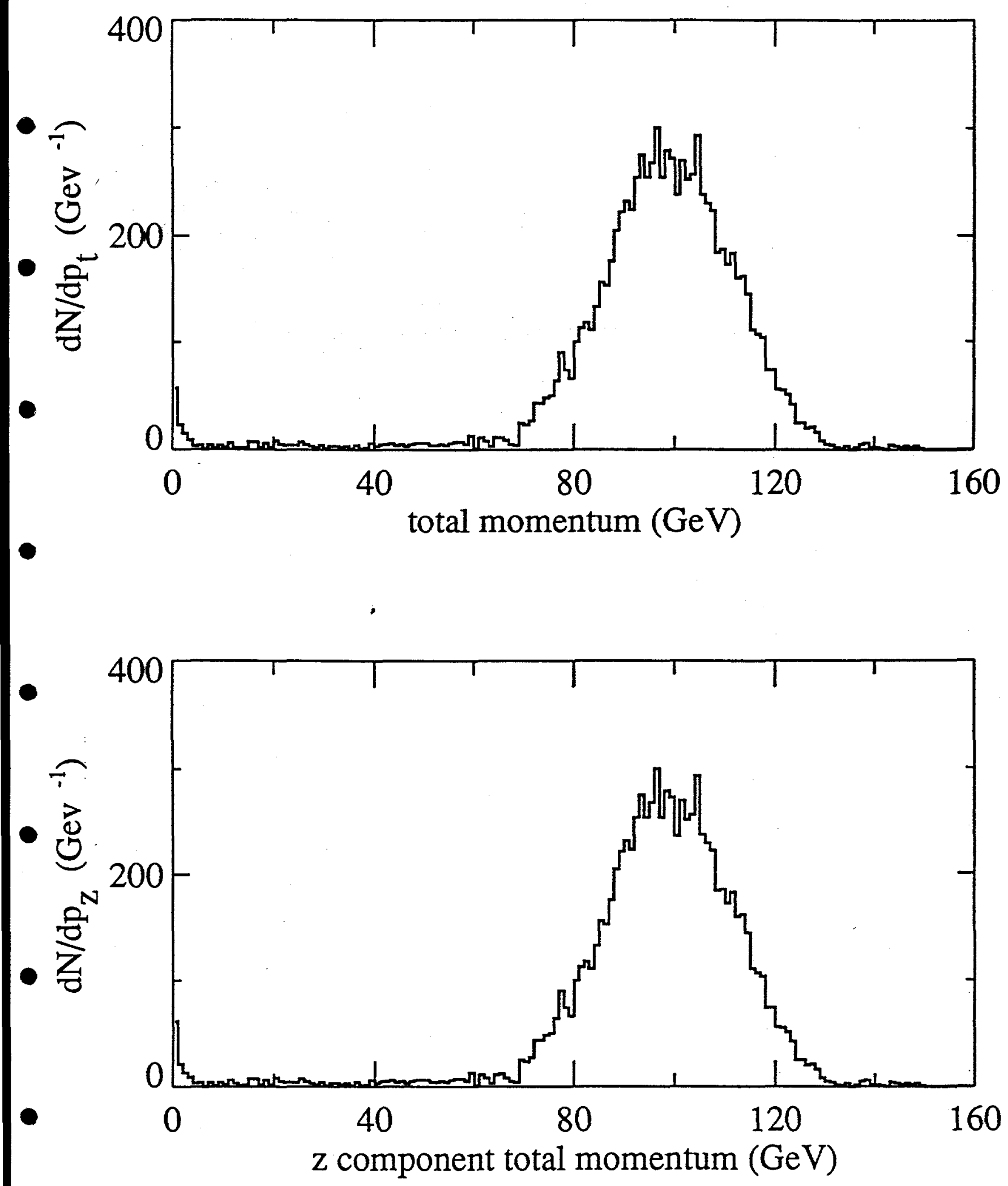

Fig 4 
Figures 3 and 4 were first shown during the talk we presented in July of this year at the workshop on beam-gas interactions at BNL. Copies of the other transparencies are included as appendix " $\mathrm{C}$ " of this report.

The first results of the simulation were enough to drive the collaboration collectively to drink in that the muon identifier was inundated with charged particles, a significant fraction of which created level- 1 triggers. The source of the charged particle flux, secondary interactions of the newly liberated nucleons, was obvious to the collaborators, and after the meeting of the muon subgroup in August, Ken Read and colleagues at UT/ORNL initiated a program to remedy this problem with HIJET. They are parametrizing the experimental results of McGaughey, et al., to produce a model that correctly incorporates the coalescence of nucleons into nuclei. We still use, and will continue to use, HIJET to generate particles such as pions and kaons that did not exist prior to the collision, but upon completion of the effort at UT/ORNL we will merge the produced particles from HIJET with the improved model of Read, et al. for the spallation of the projectile and target.

The collaborators, at least most of them, have accepted the inevitability of shielding the muon arm from particles that were produced in the primary interaction between beam and residual gas or in the secondary-interactions of those particles in material that lies along their flight paths. The determination of the optimal configuration of the shielding, by which we mean the specification of its dimensions and most advantageous deployment, is a primary objective of the simulation, and recently we presented some interesting results that are directly pertinent to our reaching this objective. The results are expressed, however, as trigger rates in various regions of the beampipe, so perhaps we should preface the presentation of results with a discussion of these various regions.

As the reader may possibly recall, the section of the beampipe that extends from the interaction point to a valve that is located approximately $80 \mathrm{~cm}$ in front of magnet D0 is warm, and we shall refer to this section of the beampipe as "region 1" for syntactical simplicity. Following region 1 is a section of cold bore that extends to a valve that is located approximately $1 \mathrm{~m}$ behind magnet CQ3C, and we shall refer to this section of the beampipe as "region 2". Following region 2 is another section of warm bore, which we shall call "region 3 ", that extends to a valve which is located approximately $1 \mathrm{~m}$ in front of Q4. Region 4 is the section of cold bore that follows region 3 .

On the following page we exhibit a re-creation of a transparency that we exhibited during the recent meeting of the muon sub-group at Kyoto. Using PISORP, Andrew Rose, a graduate student at Vanderbilt, kindly processed the phnx.dat files that were produced by PISA and provided the numbers in the second and third columns of the transparency. The results that are shown on this reproduction are significant, and we would like to discuss their meaning in some detail.

The HIJET files for hydrogen and nitrogen were used in compiling the data that appear in the tables in Fig 5. An initial ensemble was constructed from each of the two files by eliminating 


\section{Basic Numerology Courtesy ANDREW ROSE \\ Thanks, Andrew!}

$\begin{array}{ccc} & \text { Hydrogen File } & \\ \text { Region } & \text { Hits in Plane 6 } & \text { Roads* } \\ 1 & 6 & 0 \\ 2 & 1 & 0 \\ 3 & 149 & 15 \\ 4^{* *} & 67 & 7\end{array}$

Nitrogen File

Region

Hits in Plane 6

Roads*

1

0

1

2

16

3

3

310

54

$\mathbf{4}^{* *}$

64

20

* calculated using Ken Read's road finder

** the old region 4

Fig 5 
the so-called "spectator" nucleons as discussed above, so that the initial ensembles included only pions, kaons, and other particles that did not exist prior to the interaction. Although both the identity of each particle in the initial ensemble and its associated four vector were specified within the HIJET file itself, the coordinates of the interaction were set from within our routine beam_gas_evt.f. In compiling the numbers in the two tables in Fig 5 we distributed the coordinates of the interactions randomly and uniformly over one and only one region of the beampipe, with the regions being specified in column 1 of each table. We emphasize that only the coordinates of the interactions were changed; neither the identities of the particles nor the components of their four vectors were changed at any time.

Consequently, a fixed ensemble of charged particles was moved from region to region along the beampipe. PISA and GEANT were then used to calculate the number of detectors that were struck in the muon identifier by the members of this ensemble and by all the particles that they created in secondary interactions. The second column in each table of Fig 5 shows the number of hits that were recorded in plane 6 of the muon identifier. One notes at once that the largest number of hits was recorded when the interactions were distributed along region 3. The third column in each table in Fig 5, labeled "Roads" contains essentially the same information. The technical definition of a road is somewhat tangential to this discussion, and at the risk of oversimplification we encourage the reader to regard the number of roads as being the number of particles that may well be misinterpreted by the on-line software as originating in a collision between $\mathrm{Au}$ and $\mathrm{Au}$ at the interaction point. Once again one sees that the greatest threat of misidentification arises from interactions in region 3.

In some ways these results are encouraging. If continued analysis should substantiate these preliminary results then we could focus our corrective action upon regions 3 and 4 alone, thereby simplifying both the labor and the cost of suppressing the effects of interactions between beam and residual gas. We have already undertaken initial calculations along these lines, but this discussion belongs more properly in the renewal proposal than in the progress report, and perhaps it would be best to defer that discussion.

I would like to conclude this section of my annual progress report by discharging a private obligation. It would be difficult for me to acknowledge the full dimensions of the assistance rendered over the past year by Maguire's group at Vanderbilt. Their advice has been so sound; their assistance so extensive and so freely and cheerfully given as to establish an entirely new standard for excellence. Not once during my year's association with the members of that group have they sought advantage by virtue of their superior knowledge of matters computational. Not once have they exhibited irritability at answering my questions although they must have answered precisely the same questions for several dozen others prior to my involvement with PISA. Not once have they asked for any service or favor in return. There is no precedent for such public spiritedness, and there is no existing standard against which their brand of quiet, calm professionalism can be measured.

Indeed, perhaps it is a measure of the craziness of physics and life in general that we are startled when persons perform their duties with distinction, yet speak quietly and comport 
themselves modestly and with restraint. Perhaps we have become so conditioned by the bombast and by the relentless self promotion of the lost souls who populate our profession in such profusion that we no longer recognize the genuine article when we encounter it. Such melancholy speculations are correct, I believe, in the statistical, although not the absolute, sense, and it is with pleasure and with this mildly encouraging thought in mind that I express at this time and in this manner my thanks to Charles Maguire and to all the members of his group at Vanderbilt.

\section{The Calculation of the Resolution Function for the DLS}

The calculation of the Resolution Function for the DLS was discussed in detail in last year's renewal proposal. We shall not repeat that discussion here and urge the reader to review the text of last year's renewal proposal before continuing.

We have devoted a great deal of time and effort to this undertaking during the past year, and during much of it we worked closely with Roy Bossingham of LBL, whom those of us at LSU once christened "hero of the hill". Dr. Bossingham now works exclusively with the STAR Collaboration, but it is a pleasure to acknowledge his extensive work on GEANT, DECODE, and LISA.

In this report we shall use a notation that differs slightly from that established in last year's renewal proposal. In order to establish the new notation and to remind the reader of the mechanics of the procedure, we show in Figs $6-8$ copies of transparencies that we prepared for our presentation to the DLS Collaboration in late July of this year.

In Fig 6 one sees displayed the new notation. The generic symbol for momentum is "p", which is not surprising, and the symbols " $\mathrm{u}$ " and " $\mathrm{v}$ " are the generic symbols for the direction cosines of the vector momentum with respect to the $\mathrm{x}$ and $\mathrm{y}$ axes of the spectrometer reference frame (SRF). The $x$ and $z$ axes of the SRF define the horizontal plane, with the $z$ axis being the central axis of the spectrometer. The y axis points vertically upward and the direction of the $\mathrm{x}$ axis is chosen to be consistent with a right handed coordinate system. In the SRF the numerical values of " $\mathrm{u}$ " and " $\mathrm{v}$ " for a trajectory that points straight down the central axis of the spectrometer would be zero.

There are many different momenta and direction cosines, of course, and we distinguish them one from another by means of special symbols. Quantities with tildes over them represent parameters in some theorist's model, and quantities with bars over them represent the mean values of the gaussian fits to differential distributions in the laboratory system.

The reader may object that theorists are not likely to formulate their models in terms of momenta and direction cosines but rather in terms of mass, transverse momentum, rapidity, the two decay angles of the virtual photon in its rest frame, and the azimuthal angle of the production plane in the laboratory system. The objection is valid as most theorists do formulate their models in terms of these so-called "physical variables", but the objection is 


\section{Notation}

In general...

p: total momentum

U: direction cosine with respect to $\mathrm{x}$-axis (spectrometer frame)

U: direction cosine with respect to y-axis (spectrometer frame)

Specifically...

$\overline{\mathbf{D}}$ mean values of the gaussians that are the respective best : fits, for each variable, to the differential distributions

$\sigma_{p}$. standard deviation of best fit guassians for each of these $\sigma_{\mathrm{u}} \sigma_{\mathrm{v}}{ }^{\text {variables }}$

$\tilde{\mathbf{p}}$. true value for each of these variables. These correspond $\tilde{\mathbf{u}} \tilde{\mathbf{v}}$ " to the Geant parameters pleft, dcx_left, and dcy_left.

$\mathbf{N}_{\mathrm{i}}: \begin{aligned} & \text { number of incident particles. This variable } \\ & \text { corresponts to the GEANT parameter TRIG. }\end{aligned}$

$\mathbf{N}_{0}$ : number of trials successfully reconstructed by LISA.

$$
\eta: \text { acceptance } \equiv \frac{\mathbf{N}_{\mathrm{o}}}{\mathbf{N}_{\mathbf{i}}}
$$




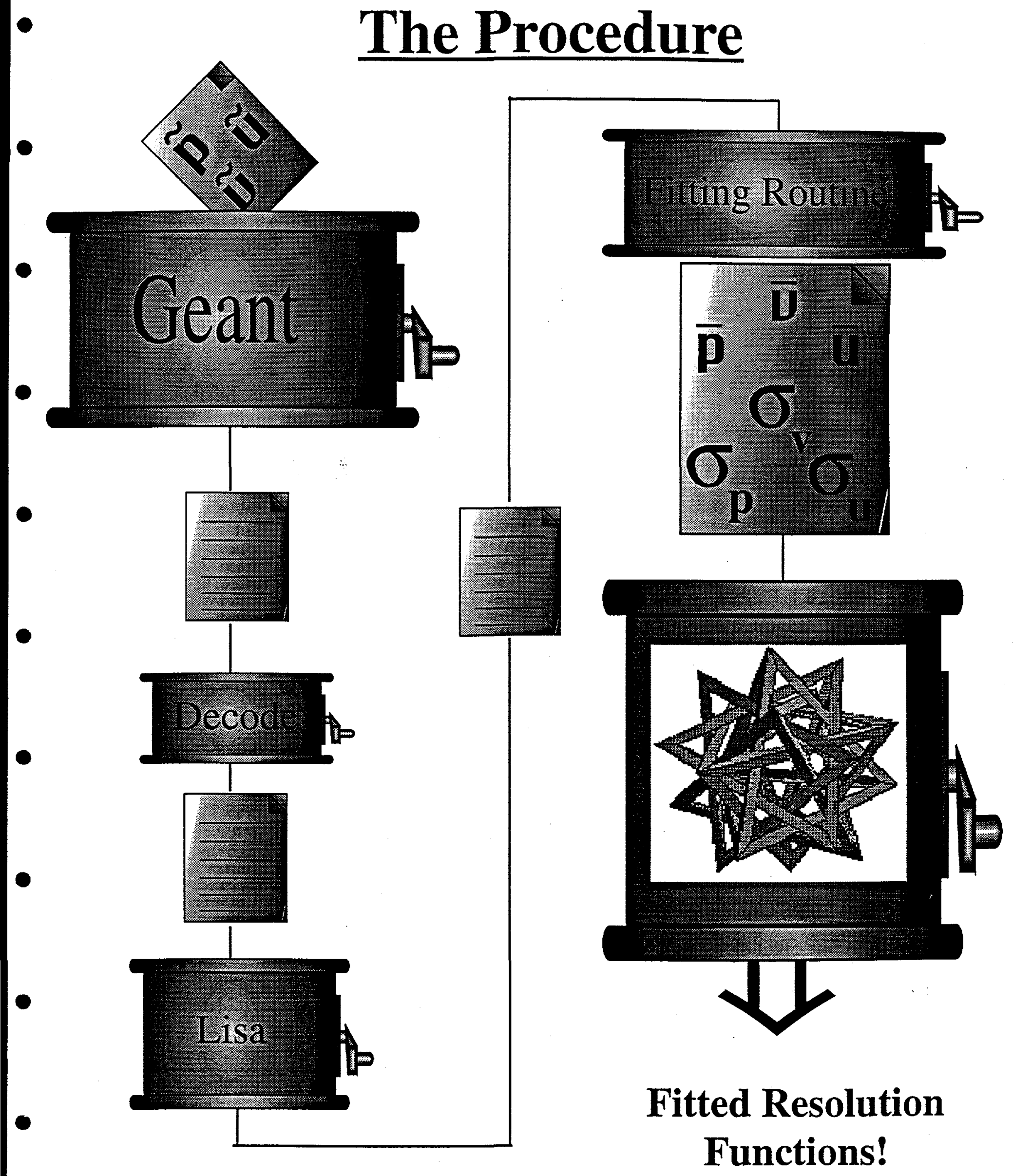

Fig 7 


\section{Notation}

$\mathbf{p}_{\mathrm{t}}$ : total momentum

$\mathbf{p}_{\mathrm{t}}^{2}=\mathbf{p}_{\mathrm{x}}^{2}+\mathbf{p}_{\mathrm{y}}^{2}+\mathbf{p}_{\mathrm{z}}^{2}: \underset{(\text { spectrometer system })}{\text { cartesian coordinates }}$

$\xi_{\mathrm{x}}: \frac{\mathbf{p}_{\mathrm{x}}}{\mathbf{p}_{\mathrm{t}}}$

$\xi_{\mathrm{y}}: \frac{\mathbf{p}_{\mathrm{y}}}{\mathbf{p}_{\mathrm{t}}}$

Fig 8 
not at all compelling. The transformation from the physical variables to the momenta of the electron and positron and their direction cosines is conceptually transparent and trivially implemented. No useful purpose is served by maintaining a distinction that is of no physical significance, and identification of $\widetilde{p}, \widetilde{u}$, and $\widetilde{v}$ as the parameters of some theoretical model involves no loss of generality.

We use conventional notation in denoting the standard deviations of gaussians, with the subscript attached to each $\sigma$ identifying the differential distribution in question. At the bottom of Fig 6 is defined the term "acceptance" as we use it in this report. The acceptance is the dimensionless number that is obtained by dividing the number of trajectories that were successfully reconstructed by LISA by the number of trajectories in the initial ensemble.

Figure 7 is Mr. Gregurich's representation of the procedure. One specifies values for $\widetilde{p}, \widetilde{u}$, and $\widetilde{v}$ in the input to GEANT, as indicated at the top of the page. The output of GEANT is used as input to DECODE, and the output of DECODE is used as input to LISA. All calculations involving GEANT, DECODE, and LISA are carried out on the alphas at LBL. The output from LISA is transmitted to LSU where differential distributions are calculated and fitted with a gaussian on our UNIX workstation. The six numbers $\bar{p}, \bar{u}, \bar{v}$, and $\sigma_{p}, \sigma_{u}$, and $\sigma_{v}$ are the output of our fitting routines. For syntactical simplicity we shall refer to this set of six variables collectively as the "normal" variables, as opposed to the acceptance, which we hereby identify as a "pathological" variable.

The reader may perhaps recognize the logo for Mathematica at the bottom of the right hand column in Fig 7. We use the multidimensional fitting routines that already exist in Mathematica to provide a global fit to the six normal variables as functions of $\tilde{p}, \tilde{u}$, and $\tilde{v}$.

Finally we call the reader's attention to Fig 8 in which two new symbols are defined. In plotting graphs we often use the Greek letter $\xi$ to denote some direction cosine in the SRF. The subscript attached to the $\xi$ denotes the axis with respect to which the direction cosine is defined.

In appendix " $\mathrm{D}$ " we list the values of $\widetilde{p}, \widetilde{u}$, and $\widetilde{v}$ for which we have calculated values for the six normal variables, as defined above, and the one pathological variable, the acceptance. The reader will probably be a bit startled at the size of this appendix. There are approximately 1400 entries, eloquent testimony indeed to the frantic pace we have maintained throughout the preceding year.

Each page in Appendix " $\mathrm{D}$ " is divided into six columns. The first column contains the value of $\tilde{p}$ in $\mathrm{GeV}$. The second and third columns contain values for $\tilde{v}$ and $\tilde{u}$, respectively. The fourth column contains the acceptance, as defined above, and the fifth column contains the statistical uncertainty in the acceptance. The sixth column contains the number of trajectories in the initial ensemble.

The pages in Appendix " $D$ " contain no values for the normal variables, largely because the normal variables behave normally and are therefore uninteresting. However, in the interests 
of preserving continuity of exposition we show in Figs $9-11$ three examples of our gaussian fits to the output of LISA. The reader has seen many plots of this type in previous progress reports, and the plots included here represent nothing that is conceptually new. Nonetheless they should remind the reader of the general characteristics of the DLS and of the quality of our fits. If the reader should wish to view more plots of this type we will be delighted to provide them in any desired quantity.

There is a minor problem with the wording of the titles on these graphs to which we should perhaps call the reader's attention. The symbol " $p_{t}$ " in the title stands for total momentum, not transverse momentum, and the symbol should actually have been written as " $\vec{p}$ " instead of " $p_{t}$ ". Similarly the symbols " $\xi_{x}$ " and " $\xi_{y}$ " in the titles should have been written " $\widetilde{u}$ " and " $\widetilde{v}$ ", respectively. The symbols " $\xi_{x}$ " and " $\xi_{y}$ " on the abscissae and ordinates of the plots themselves are correct as written. Finally the symbol " $p_{t}$ " on the ordinate and abscissa of the plot on the right stands for total momentum, not transverse momentum.

As the reader may perhaps already know from personal experience multidimensional fitting is an undertaking of complexity sufficient to render trivial by comparison the non-linear fitting illustrated in Figs 9 - 11. We have been fortunate in that much of the labor was carried out by the authors of Mathematica. Our tasks were to construct and to maintain the data set in which are stored values for the acceptance and the six normal variables and to specify the basis functions from which $\underline{\text { Mathematica }}$ constructs the fitted functions.

Initially we used simple polynomials in $\widetilde{p}, \tilde{u}$, and $\widetilde{v}$ as the basis functions, but the results were somewhat disappointing. The most advantageous basis functions for our purpose are well known to be the Chebyshev polynomials, and we have been using Chebyshev polynomials as basis functions since July.

The output from Mathematica is a set of six linear combinations of Chebyshev polynomials, with each member of the set representing one of the functions $\bar{p}(\widetilde{p}, \widetilde{u}, \widetilde{v}), \bar{u}(\widetilde{p}, \widetilde{u}, \widetilde{v}), \bar{v}(\widetilde{p}, \widetilde{u}, \widetilde{v})$, $\sigma_{p}(\widetilde{p}, \widetilde{u}, \widetilde{v}), \sigma_{u}(\widetilde{p}, \widetilde{u}, \widetilde{v}), \sigma_{v}(\widetilde{p}, \widetilde{u}, \widetilde{v})$. In Figs $12-17$ we show a sample of six graphs in which the fitted function is plotted against data. The tildes have been omitted both in the titles and also in the abscissae of the plots, and we call the reader's attention to this omission.

We should perhaps emphasize that in general the agreement between fitted function and data is not as impressive as might be inferred from these graphs. Indeed, if these graphs were typical of all the graphs at our disposal, we would be submitting a concluding report at this time, not a progress report. Figures $12-17$ are best regarded as a proof of principle, not as typical results. In addition the reader will note that we present no comparison between fitted function and acceptance, the pathological variable. The reason for this painful omission is that we no longer believe the acceptance can be fit in the manner outlined in the preceding paragraphs. Other techniques will be required, but this discussion is more properly a part of the renewal proposal, and on this note we conclude our discussion of activities that pertained to the DLS. 

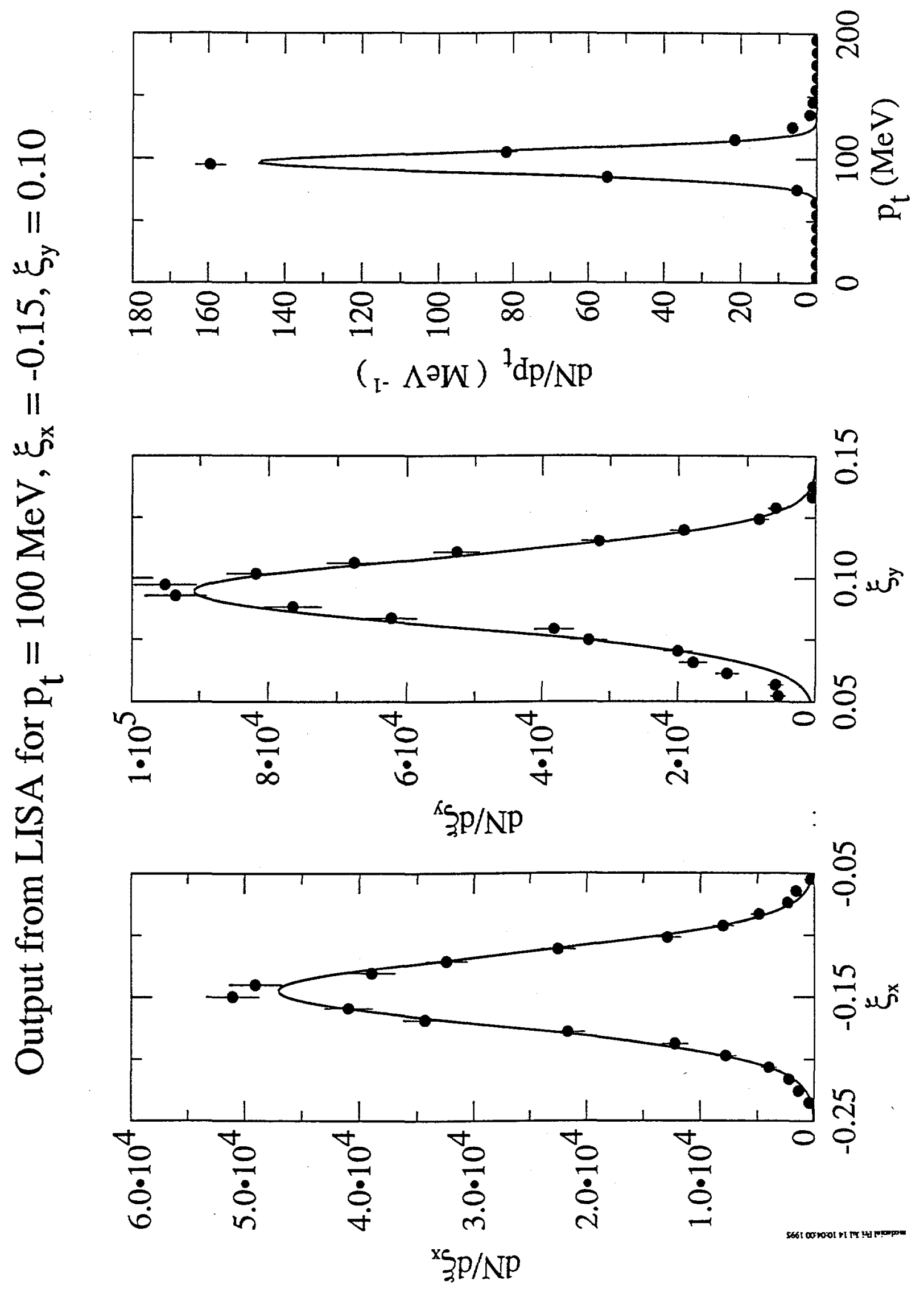

Fig 9 

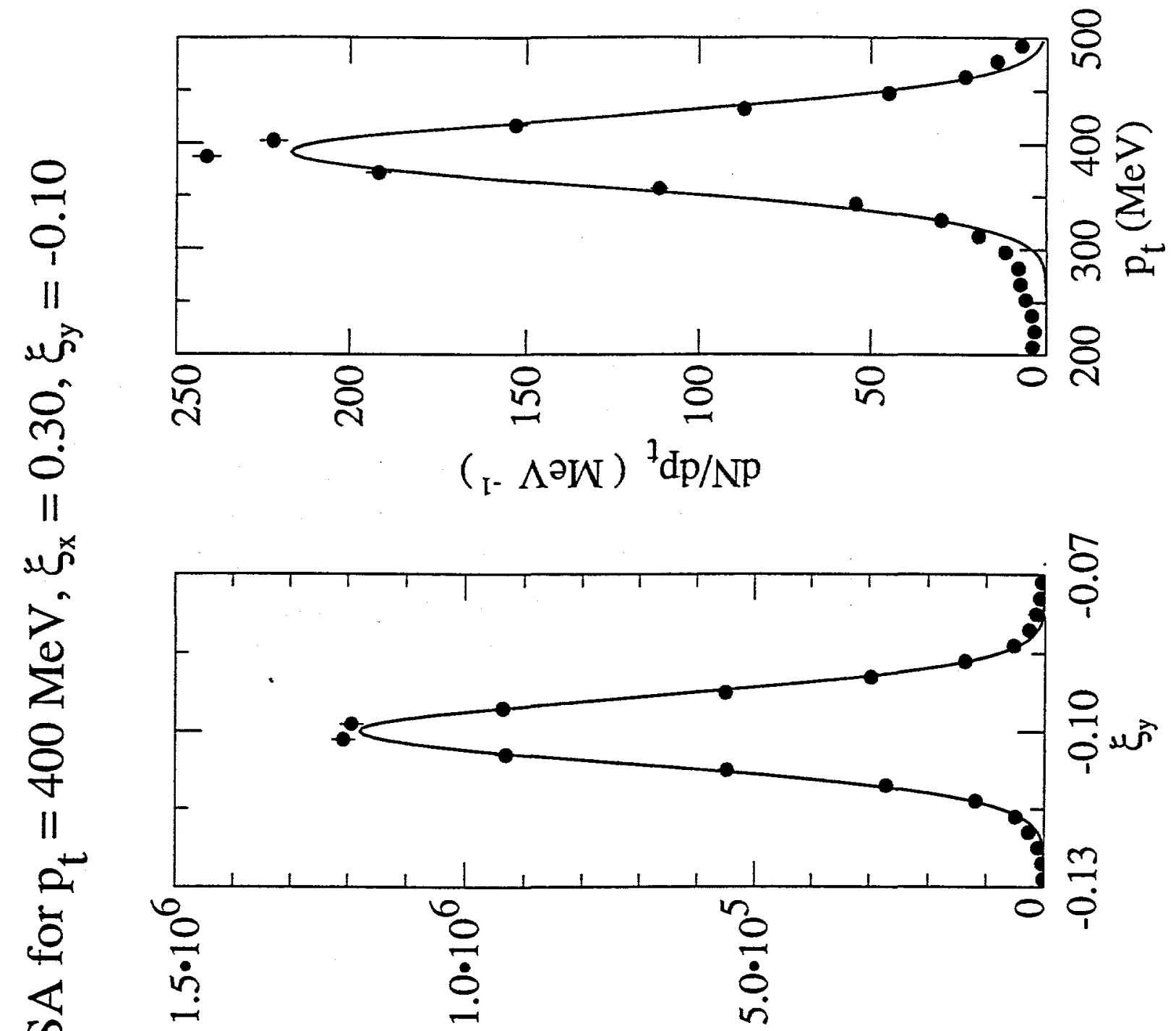

年

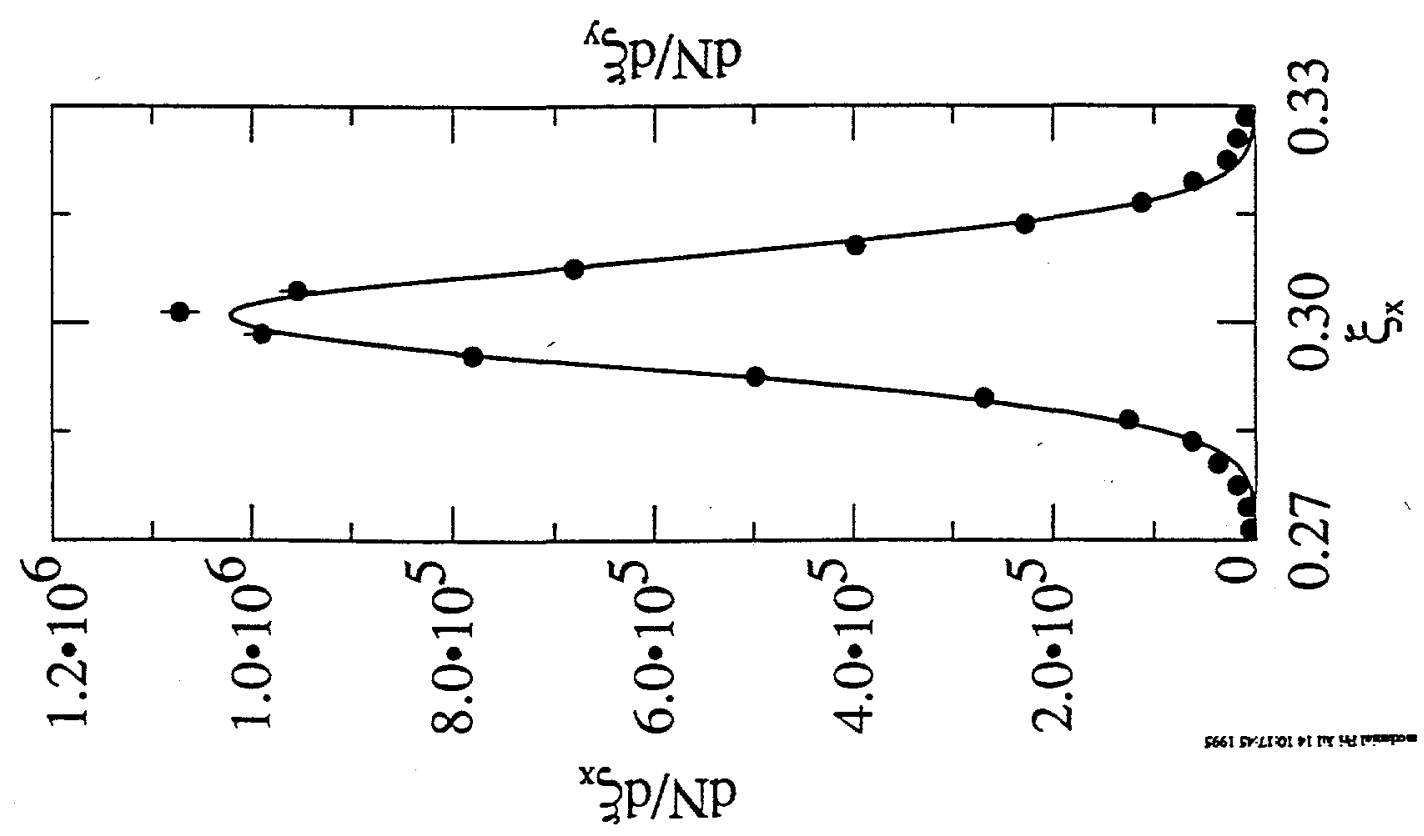

Fig 10 


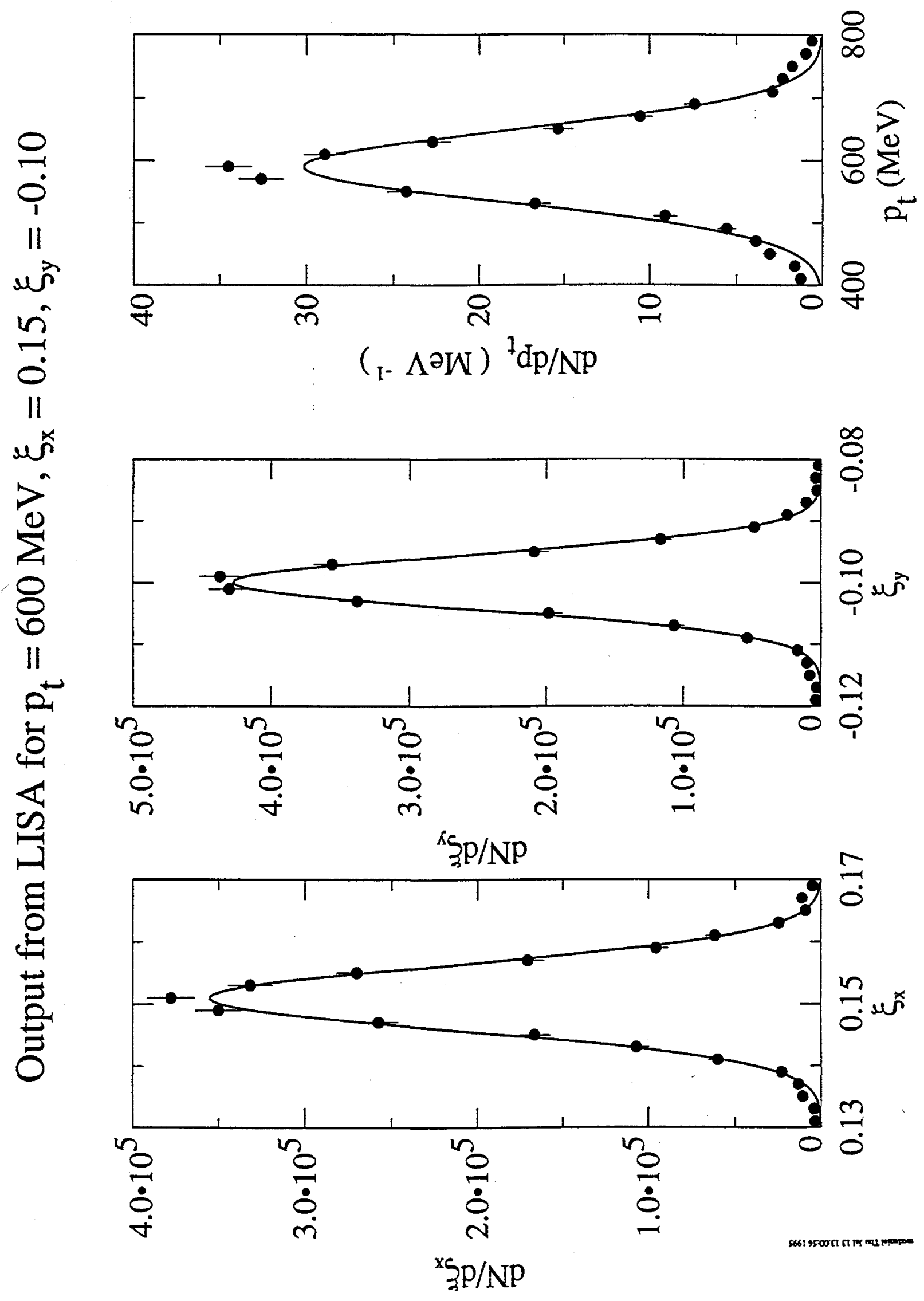

Fig 11 


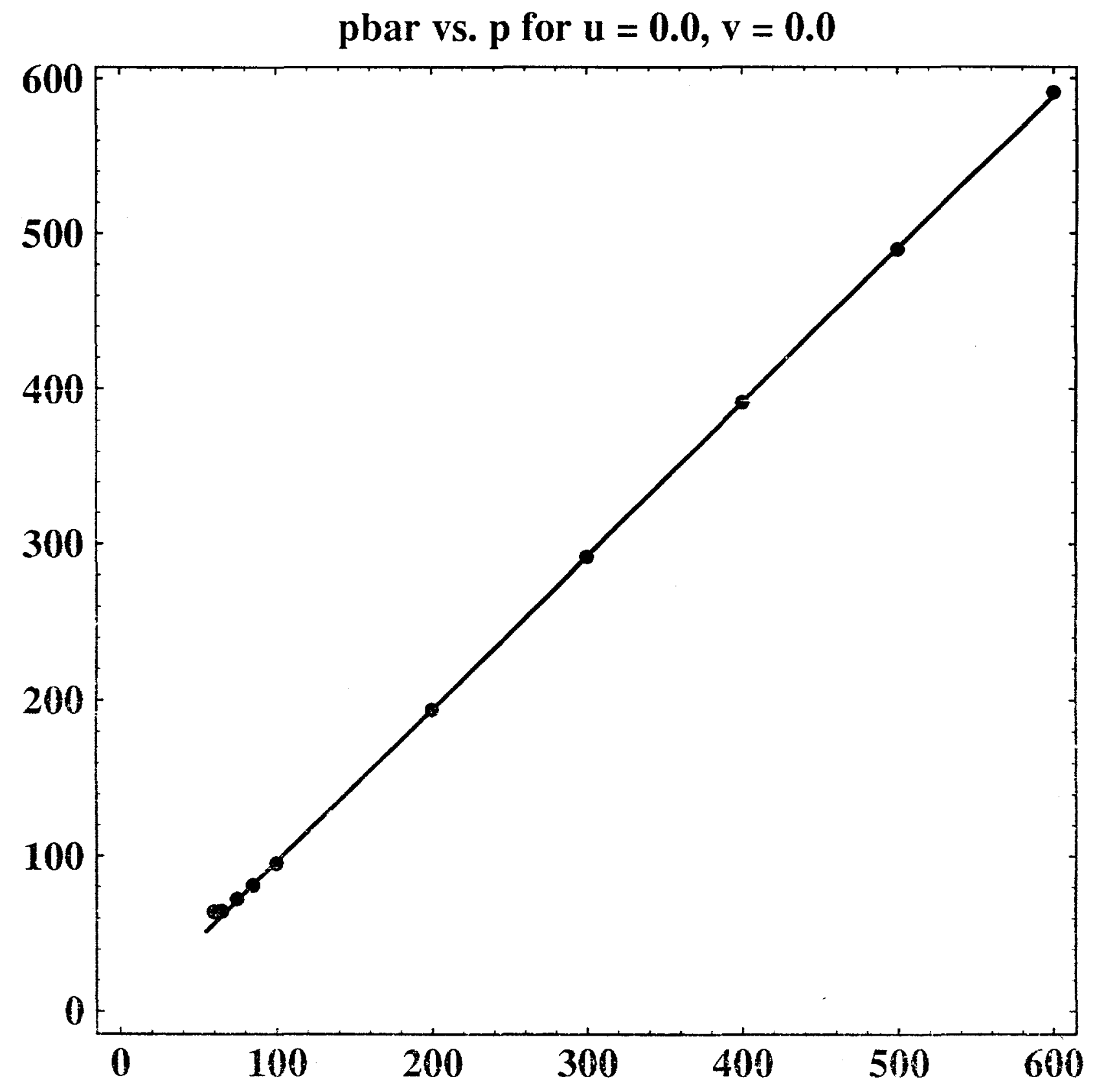

Fig 12 


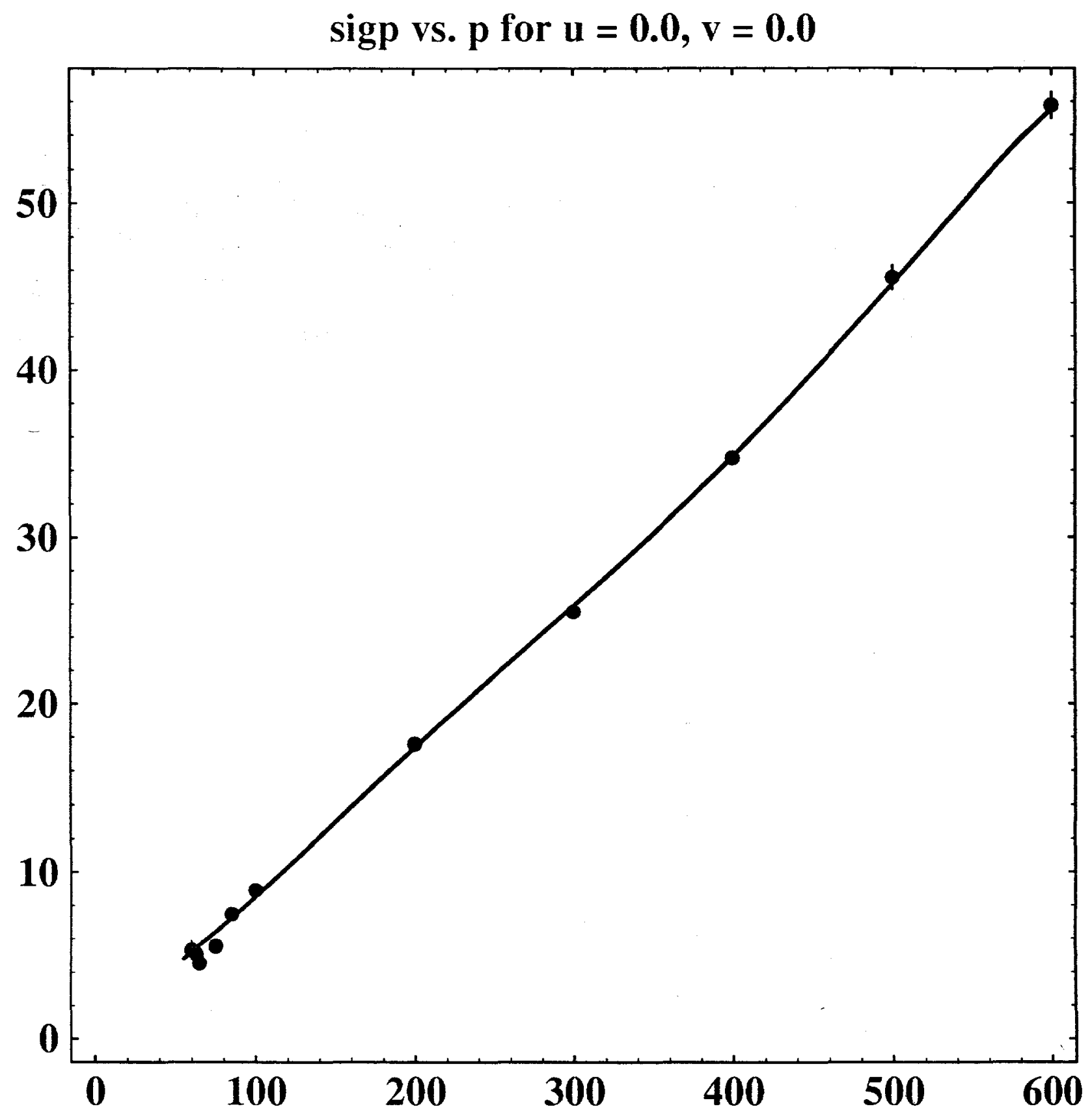

Fig 13 


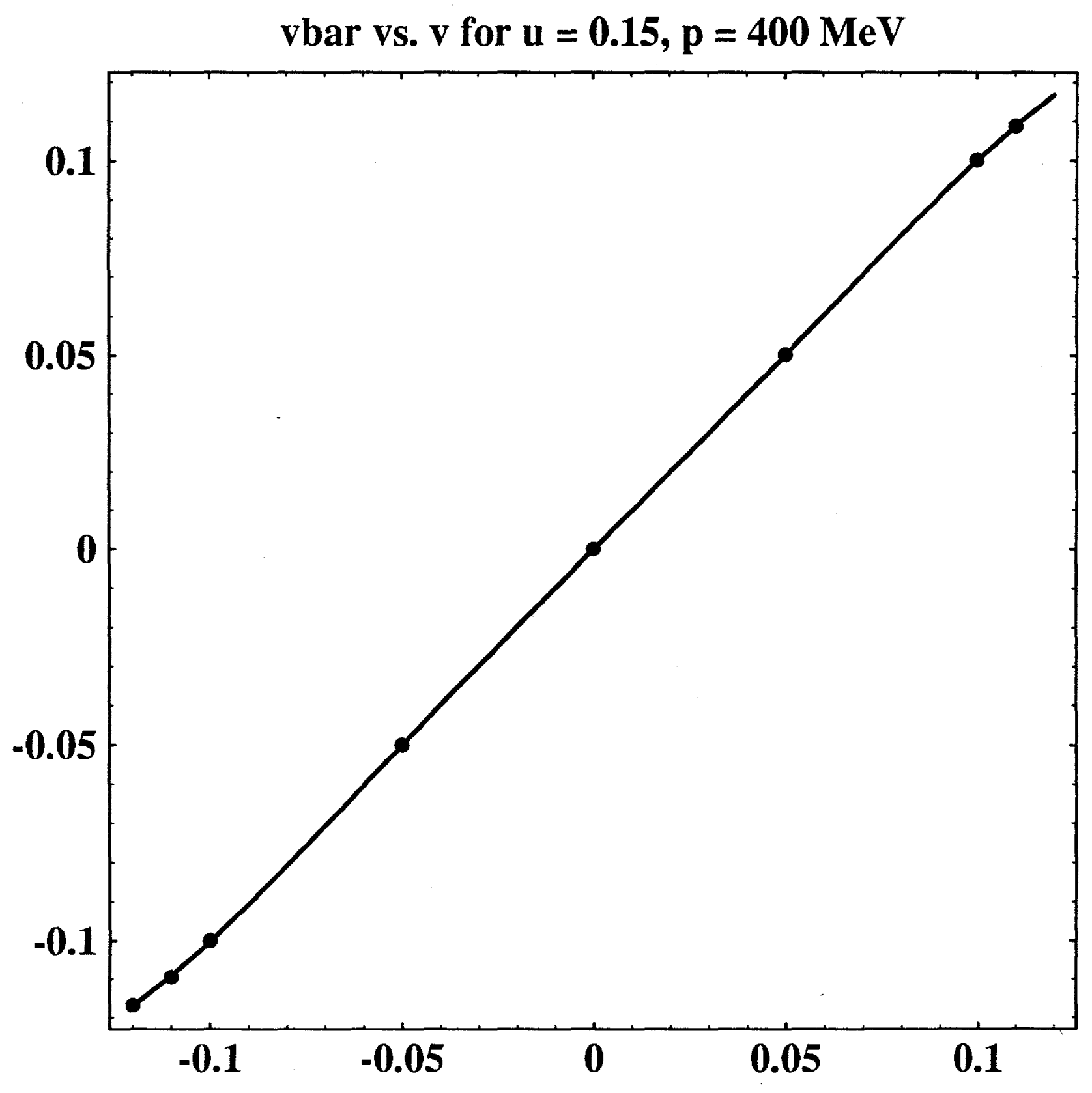

Fig 14 


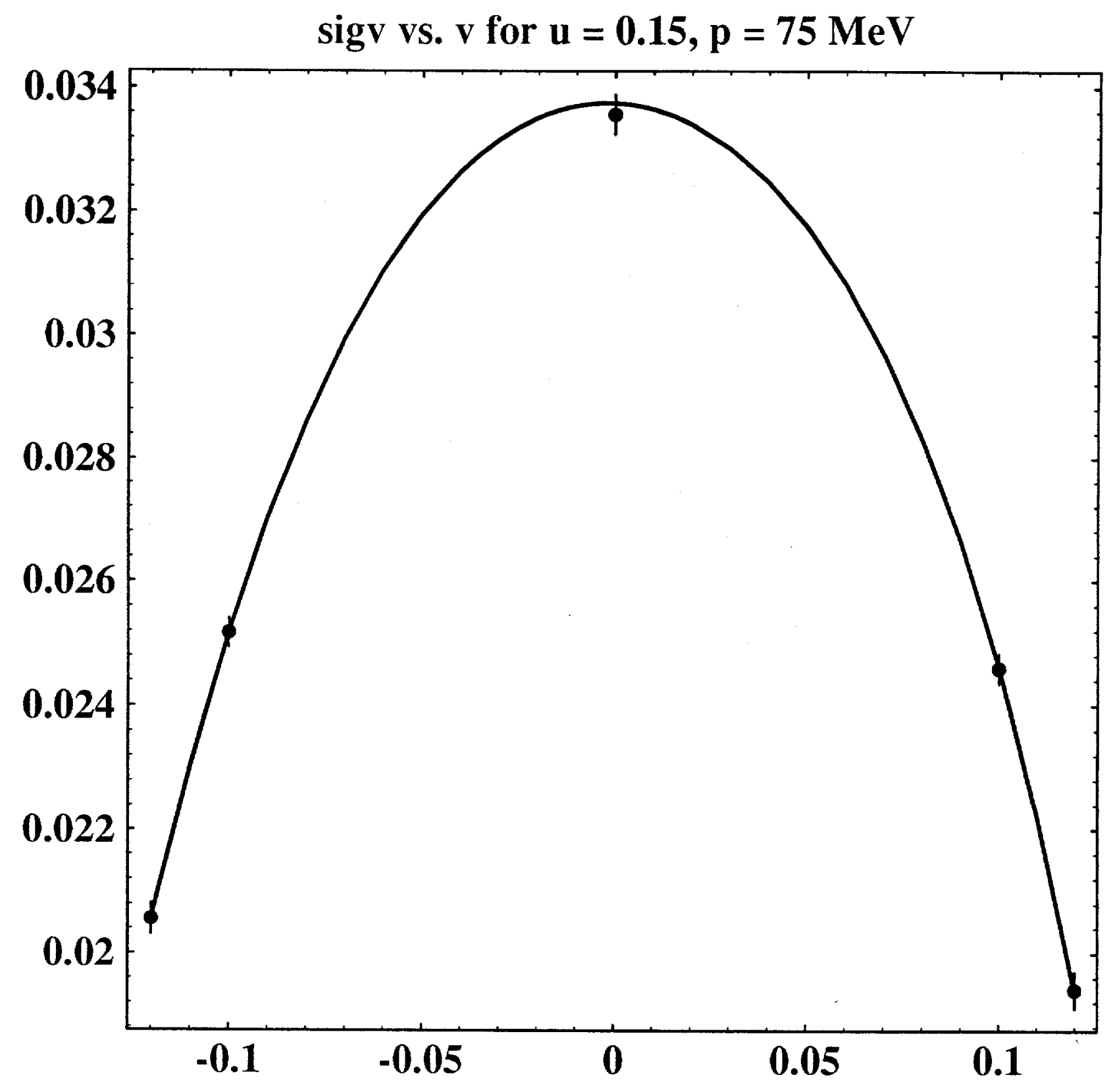

Fig 15 


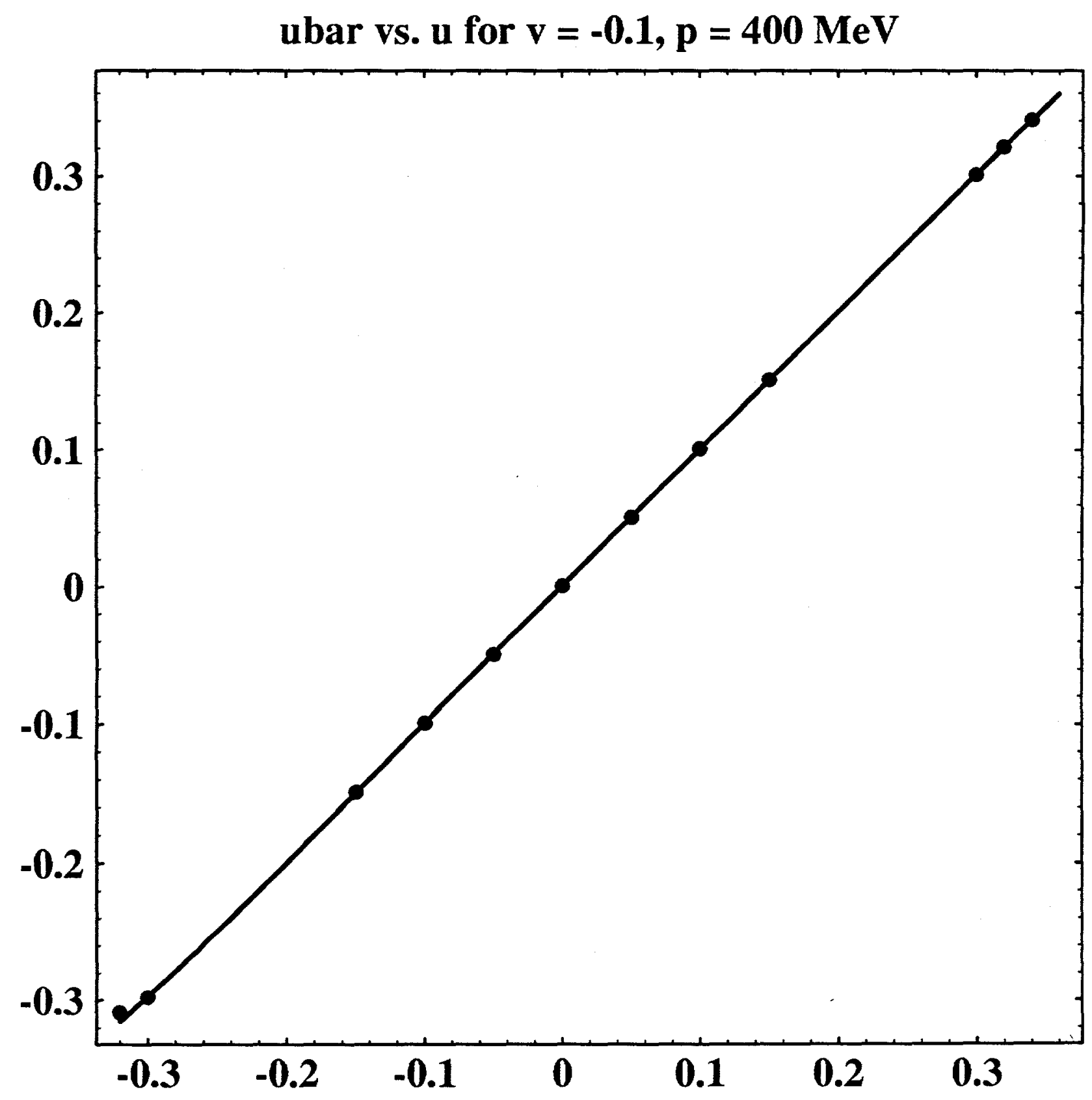

Fig 16 


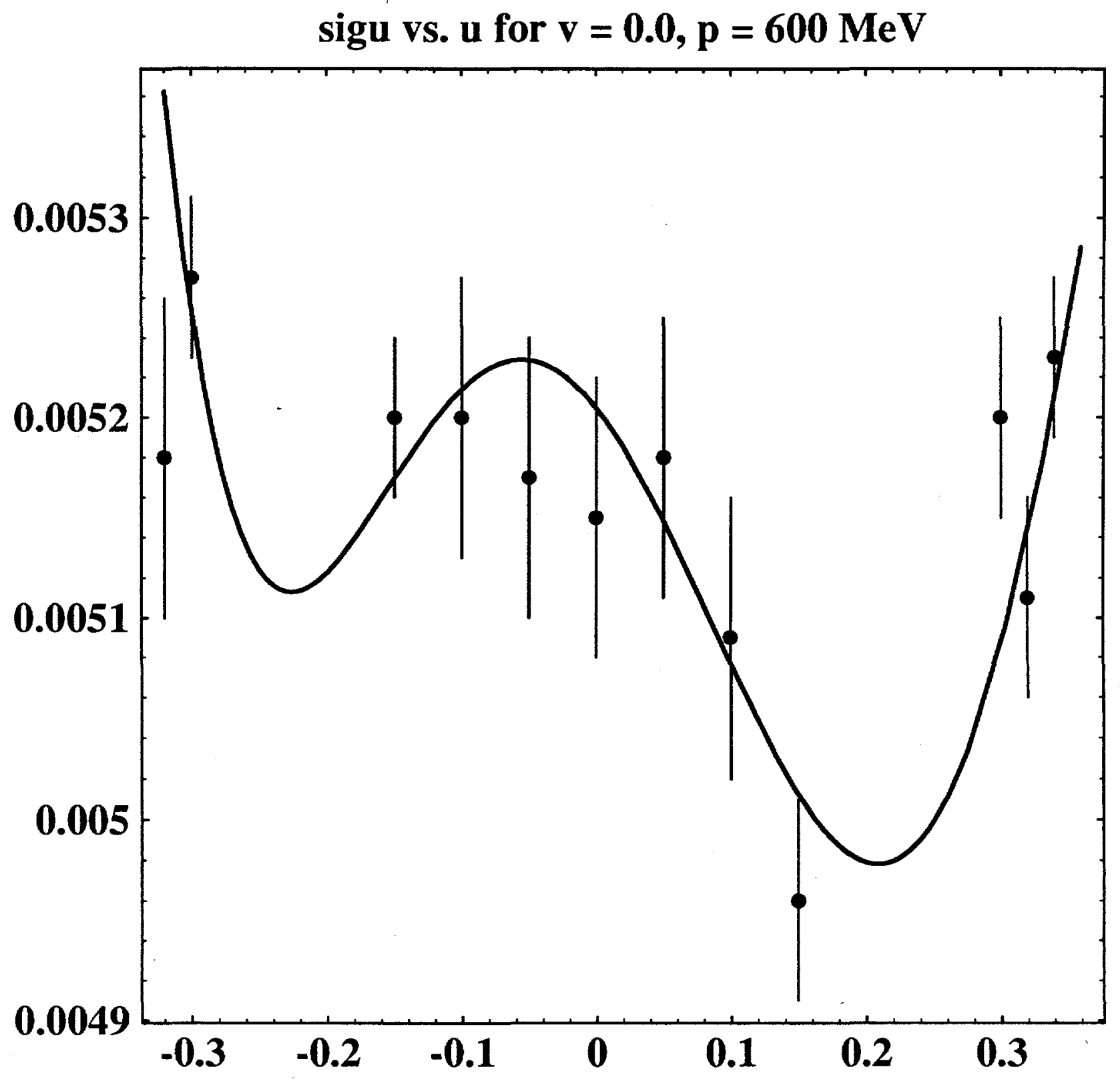

Fig 17 


\section{The E866 Collaboration}

During the preceding year Zhi-fu and Ying-chao Wang have contributed significantly to experiment E866 at Fermilab. They designed and built three wire chambers, which we denote by $Y-Y^{\prime}, U-U^{\prime}$, and $V-V^{\prime}$. With an active area of 46 inches by 40 inches, these chambers are much larger than those we designed and built for the DLS Collaboration, and for this reason their design and construction was correspondingly more challenging. Each chamber contains two anode planes and three cathode planes. The cathode planes are gold plated mylar. Each of the two anode planes in the $Y-Y^{\prime}$ chamber contains 160 sense wires and 160 field wires. The $U-U^{\prime}$ chamber and the $\mathrm{V}^{-\mathrm{V}^{\prime}}$ chamber each contain 200 sense wires and 200 field wires per plane.

Using a collimated source of $\mathrm{Sr}^{90}$ the Wangs checked the response of each wire in each plane for uniformity, replacing those wires that responsed in a manner that was abnormal or atypical. The results of their meticulous and painstaking work are impressive indeed. All three chambers have been extensively tested, and as of the present time the analysis of data logged with the $Y-Y^{\prime}$ chamber has been completed. The $Y-Y^{\prime}$ chamber responds with an efficiency of $98 \%$. Its high voltage plateau is no less than 250 volts long, and, best of all, its uncommonly low dark current permits the threshold of the associated electronics to be set at low levels. There is no evidence for cross talk, radio frequency pickup, or oscillation in the associated electronics. The analysis of the data logged with the U-U' chamber and the $V-V^{\prime}$ chamber has not been completed yet, but no one associated with the effort expects the performance of those two chamber to differ significantly from the performance of the $Y-Y^{\prime}$ chamber.

In the pages that follow this text is shown a series of ten photographs depicting the Wangs in various stages of their activities. In photographs 1 and 2, Zhi-fu and Ying-chao are shown in an early stage of their activities. They are laminating the circuit boards for one of the anode planes prior to winding. In photograph 3, Ying-chao is seen measuring the tension in the wires. In photograph 4 , Zhi-fu is shown soldering capacitors onto one of the anode planes of the $Y-Y^{\prime}$ chamber. In photograph 5, Ying-chao is shown installing one of the connectors into which the amplifiers are plugged. In photograph 6 , Zhi-fu is shown checking the continuity of the solder joints. Note his reflection in the gold plated mylar foil. In photograph 7, Zhi-fu is shown assembling the $\mathrm{Y}^{-\mathrm{Y}^{\prime}}$ chamber, and in photograph 8 , he is shown checking each wire for a proper output. In photograph 9 , the $\mathrm{V}-\mathrm{V}^{\prime}$ chamber, having passed all applicable tests, is shown standing on the floor awaiting shipment to Fermilab. Finally, in photograph 10, Ying-chao is shown standing beside the $\mathrm{Y}_{-} \mathrm{Y}^{\prime}$ chamber which is being subjected to additional tests.

At the time of writing the Wangs are on-site at Fermilab installing the chambers in the beamline. Zhi-fu will present a progress report to the collaboration during the next regularly scheduled meeting on December $15-16$. 


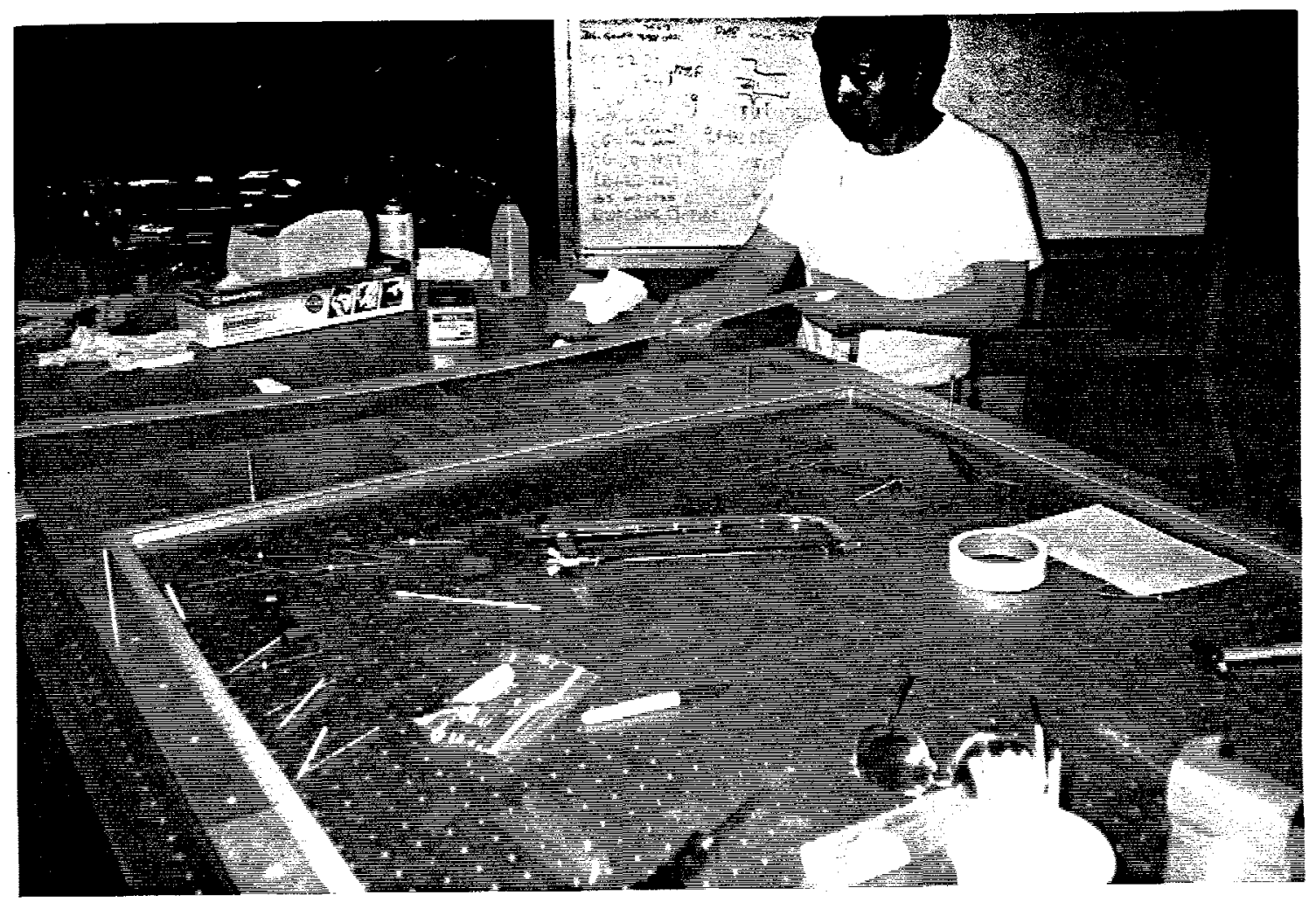

Photograph 1

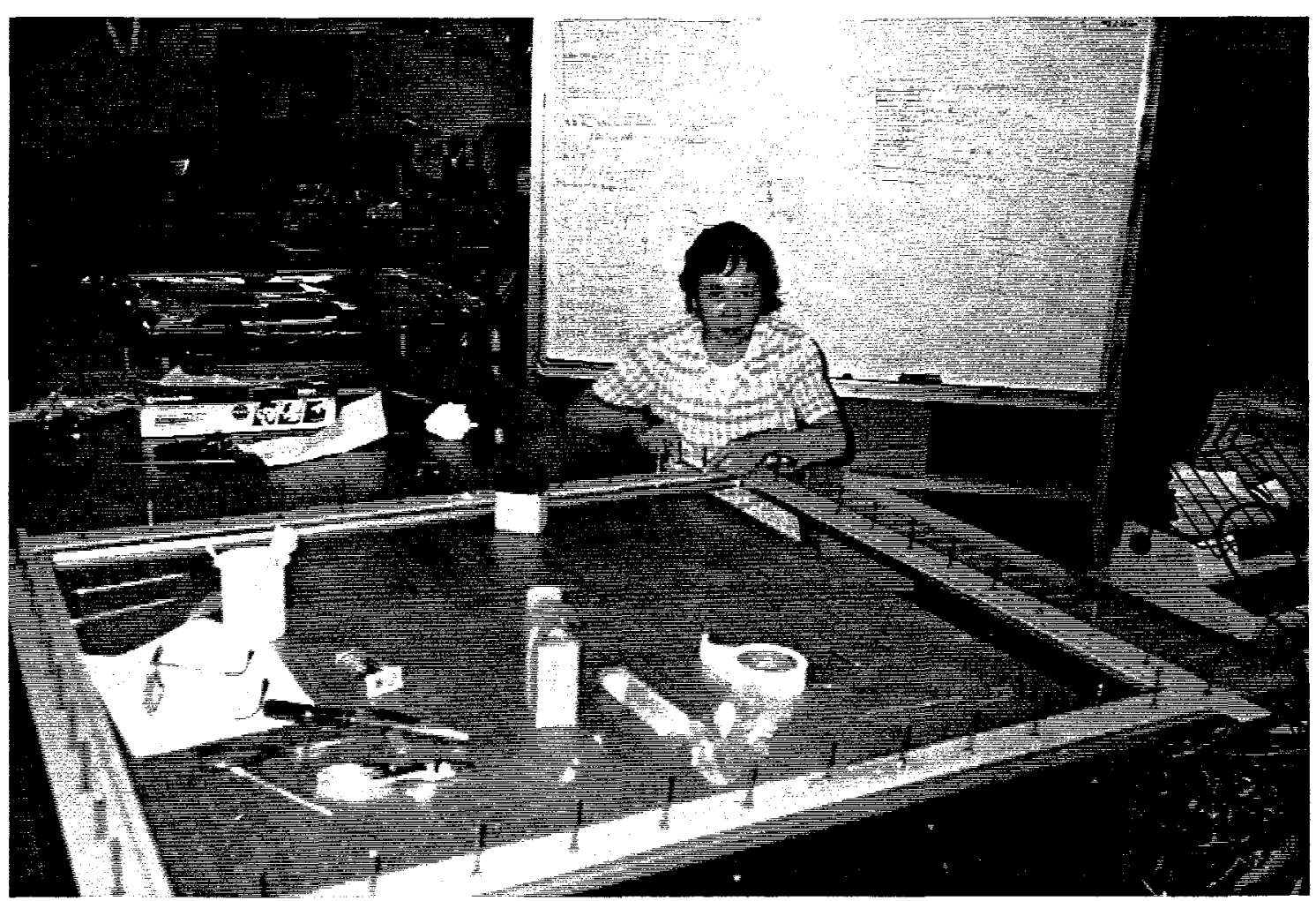

Photograph 2 


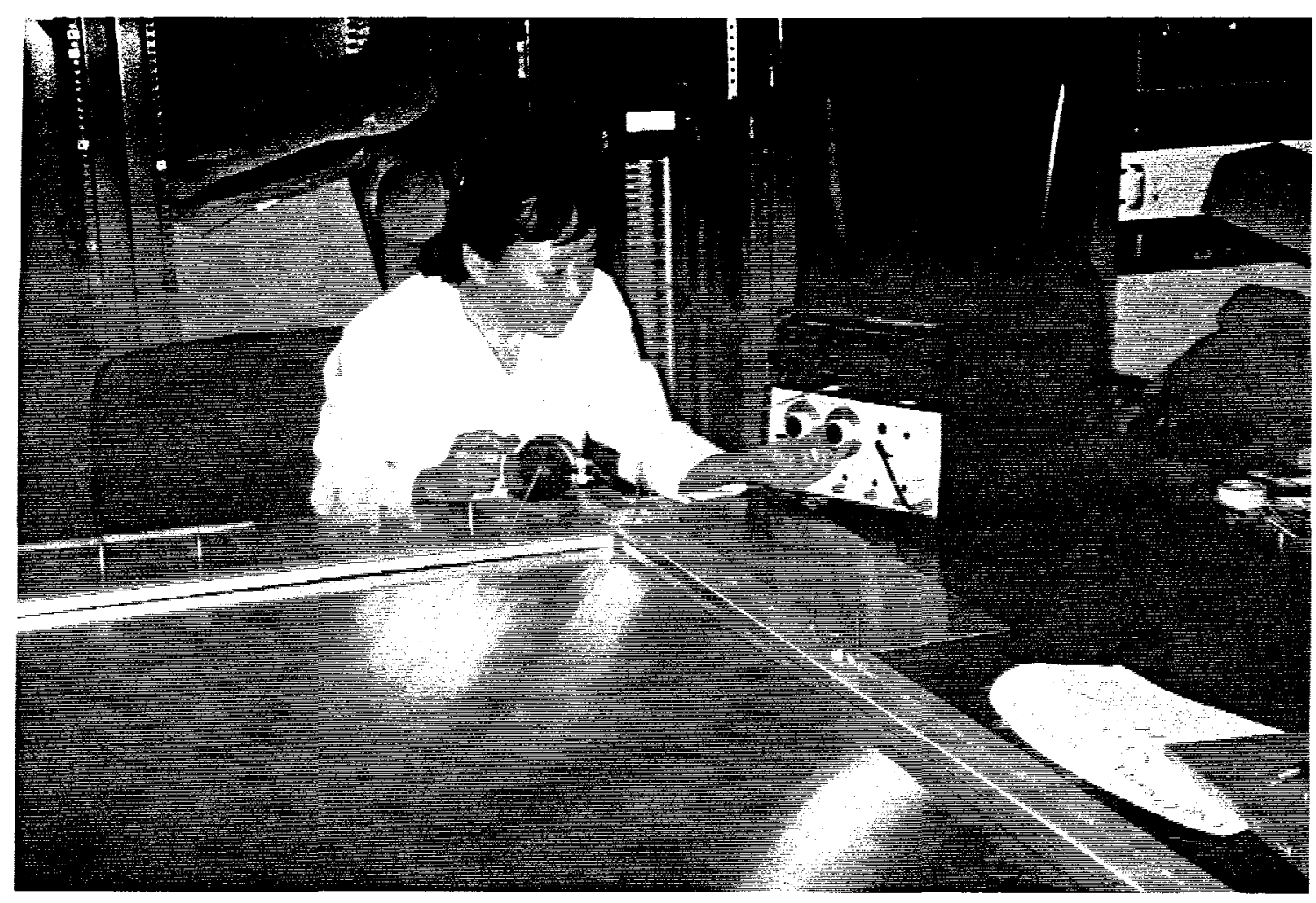

Photograph 3

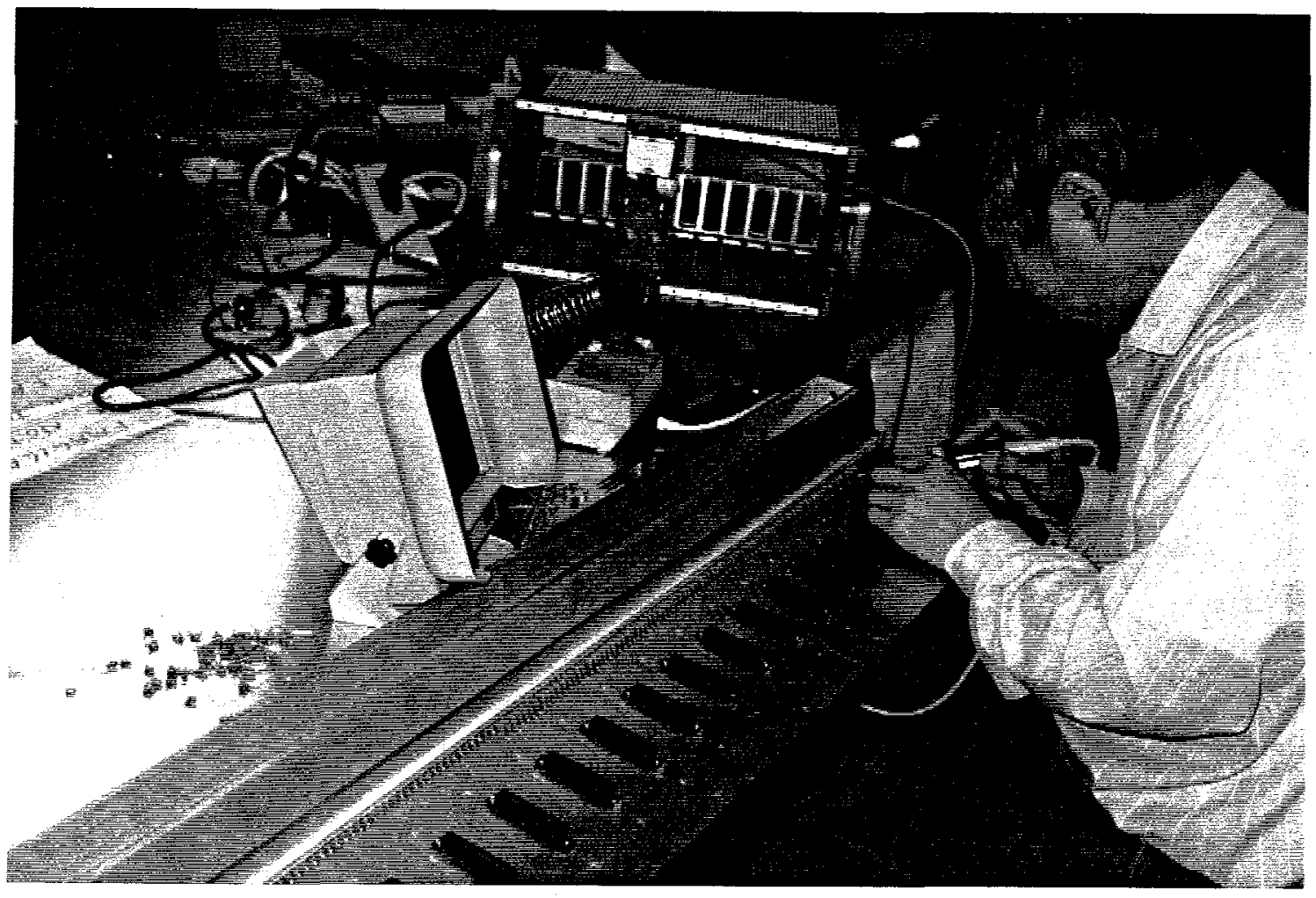

Photograph 4 


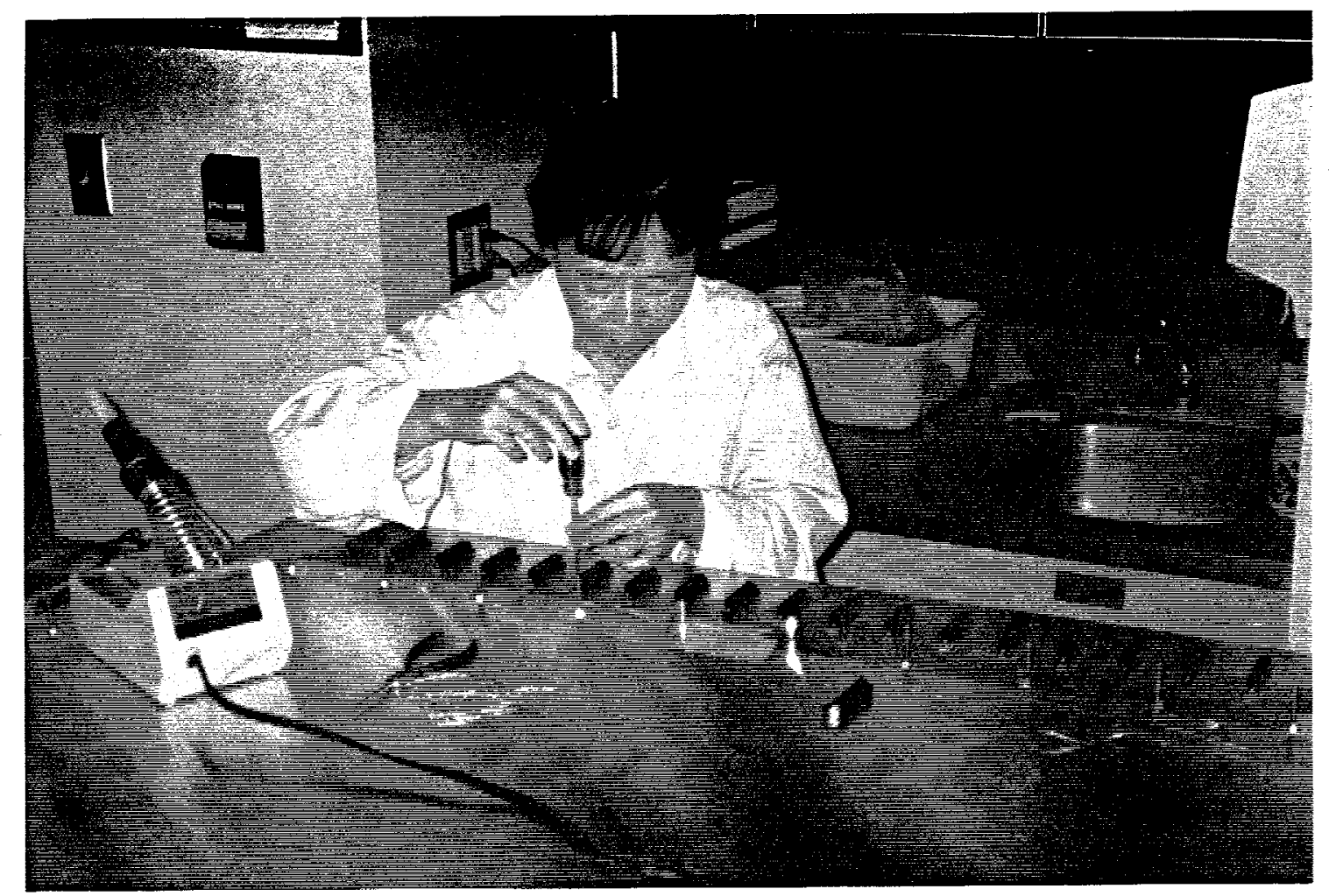

Photograph 5

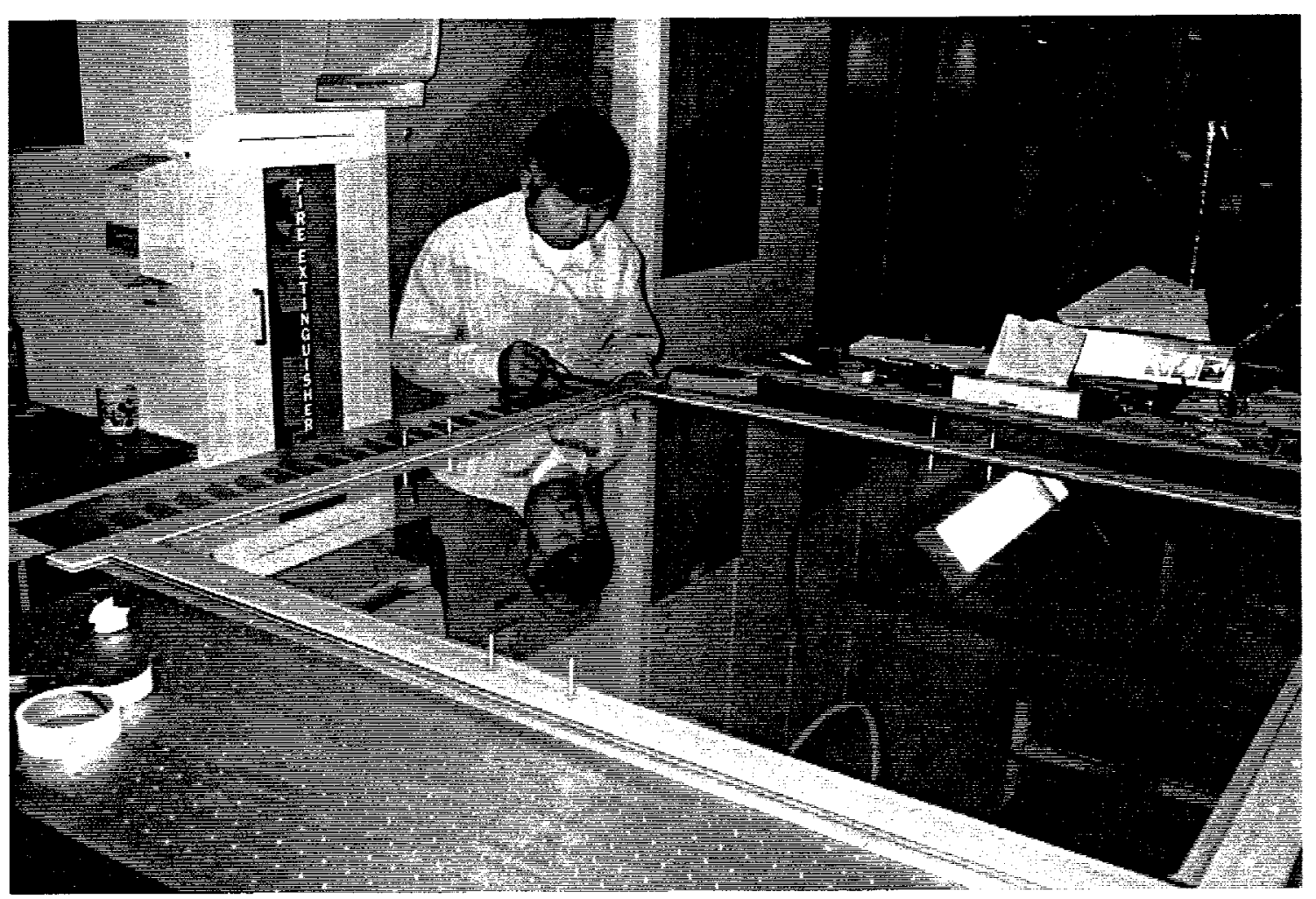

Photograph 6 


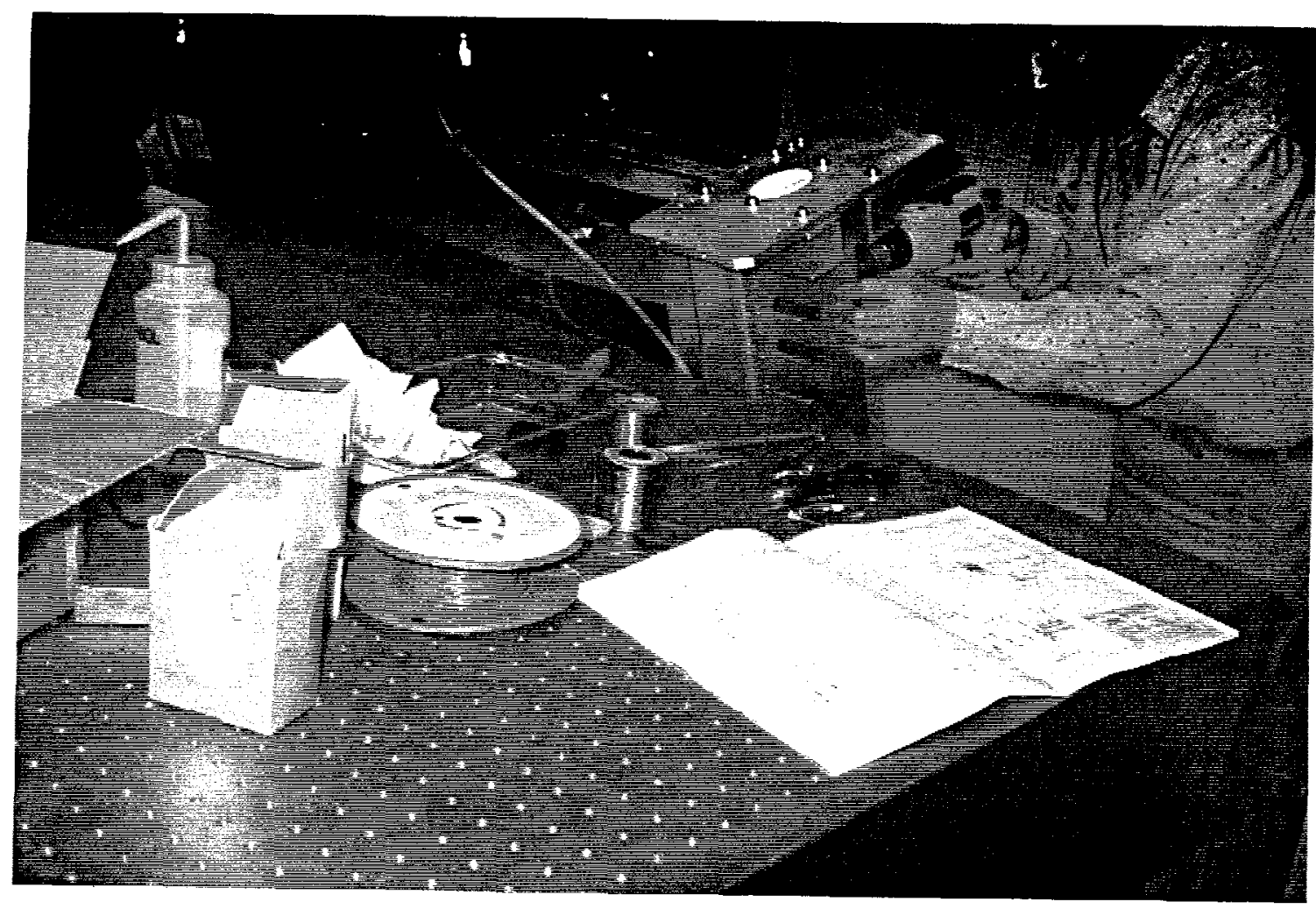

Photograph 7

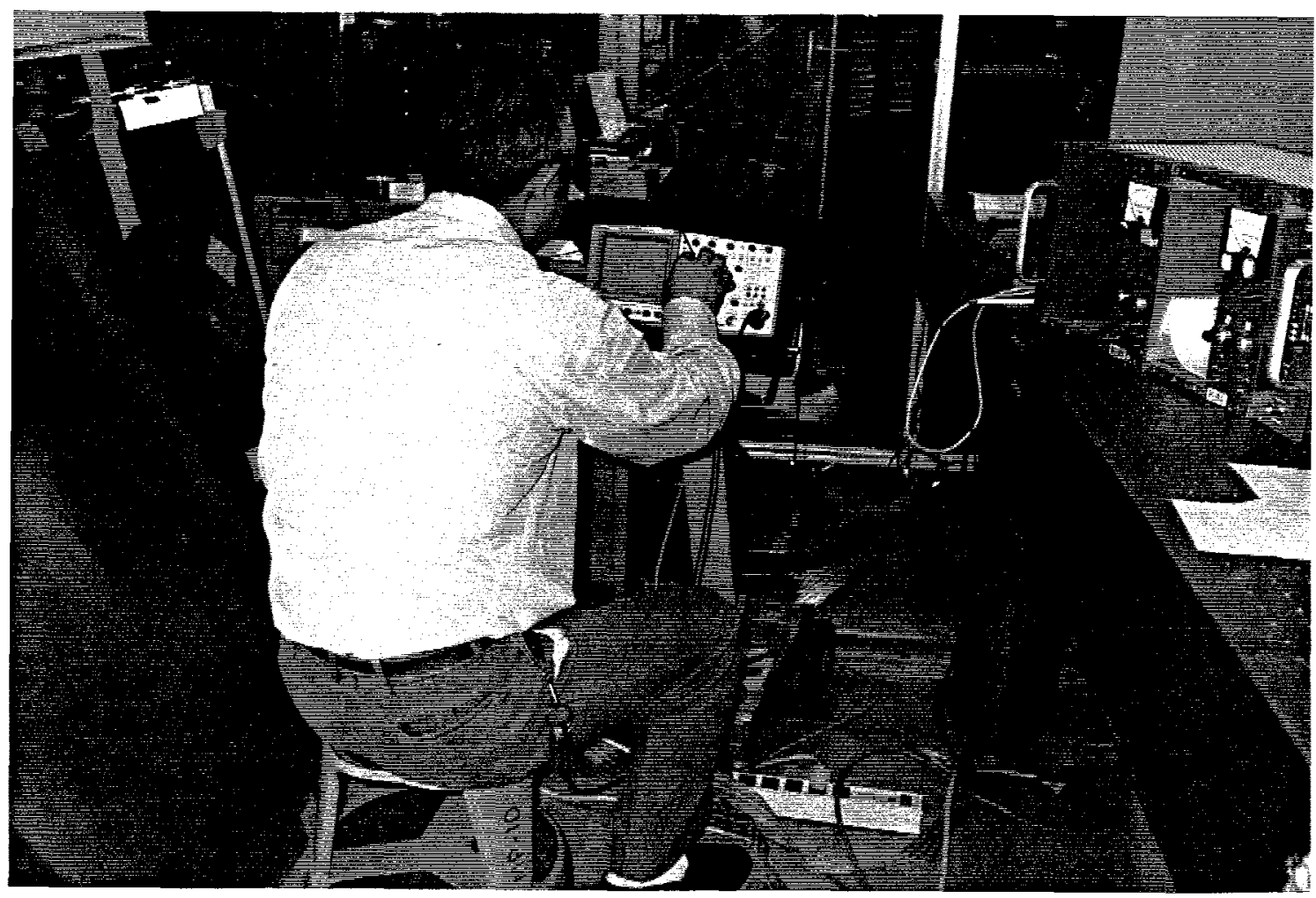

Photograph 8 


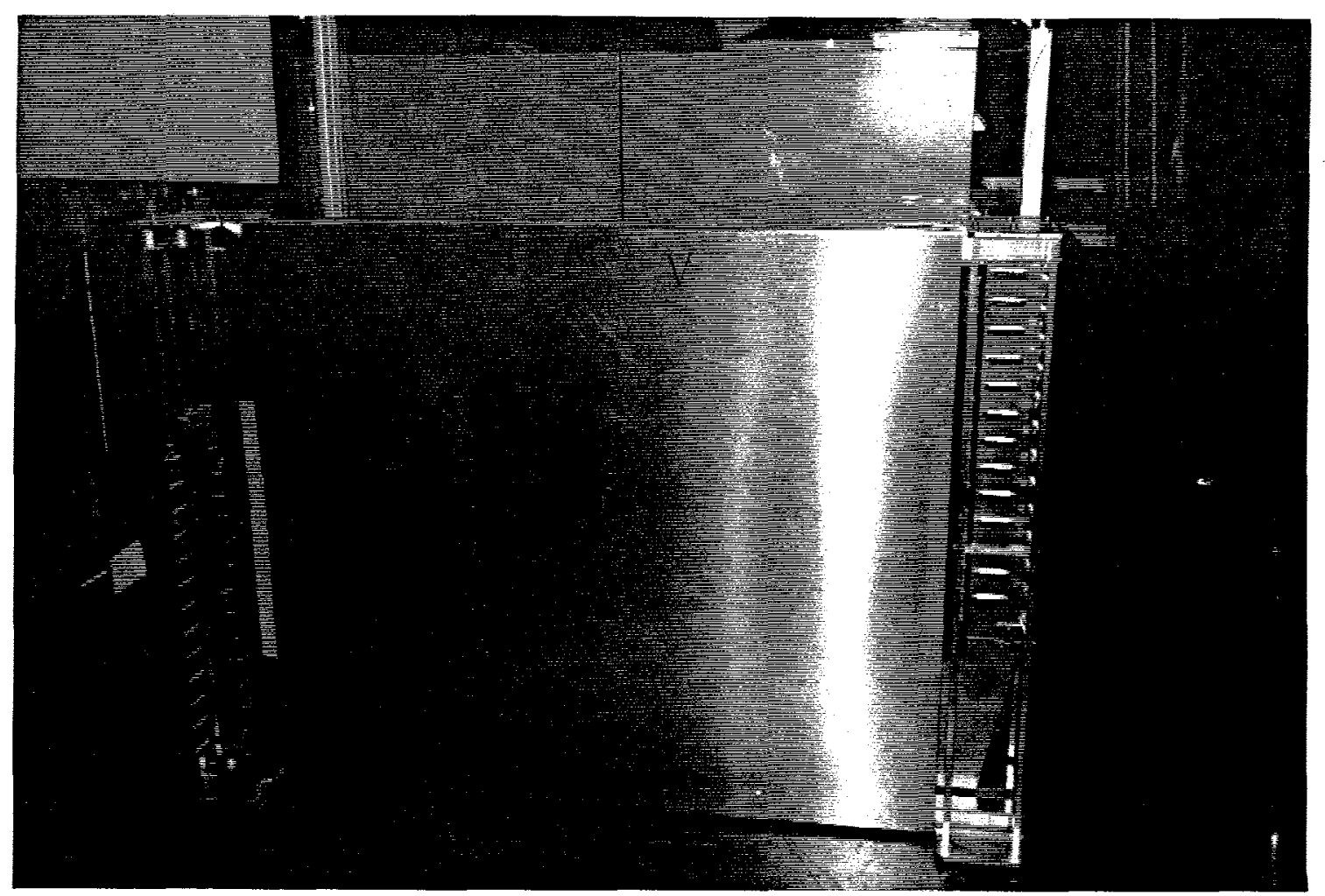

Photograph 9 


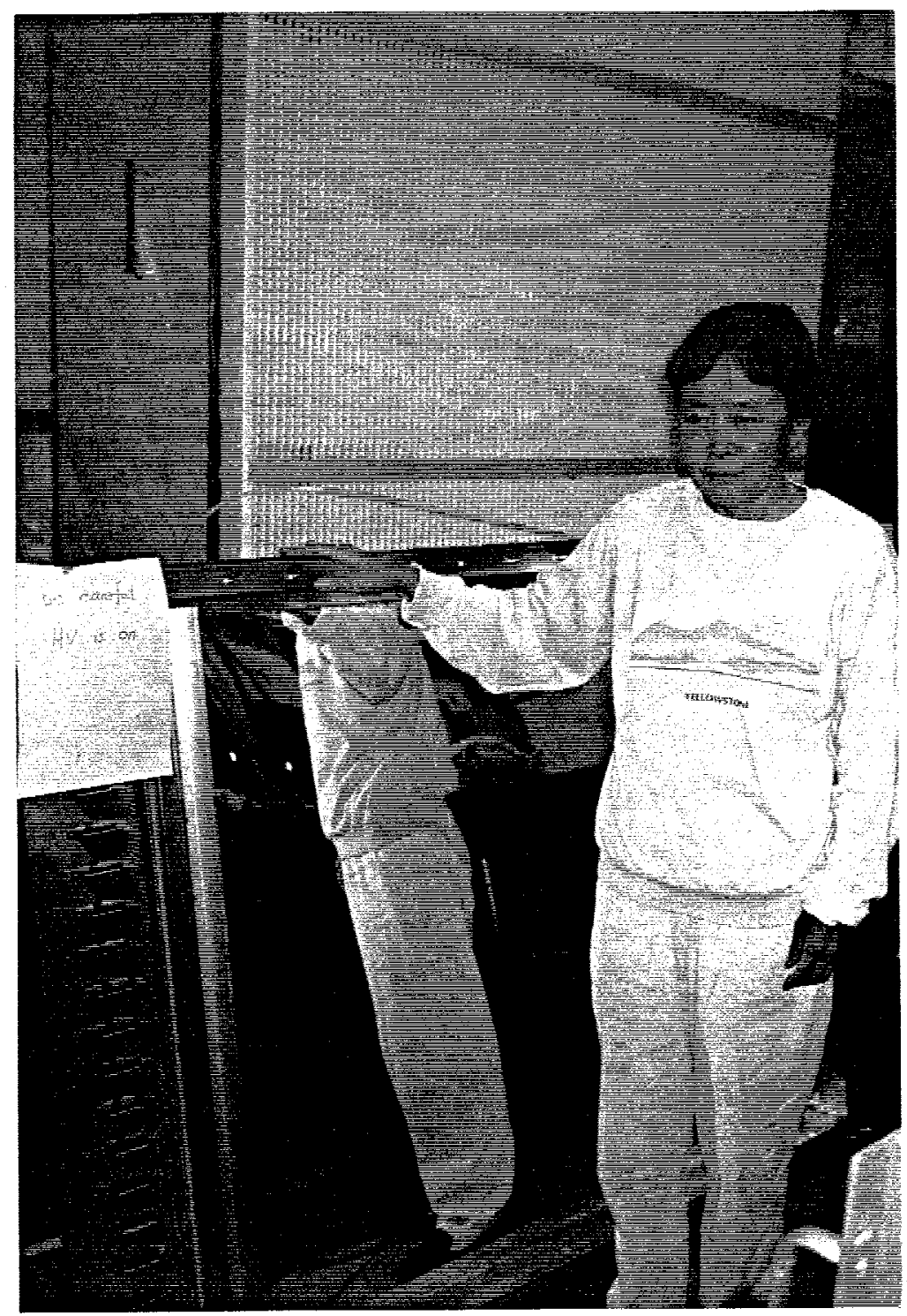

Photograph 10 
Appendix A

the Fortran subroutine rhic_magnet_install.f 
C

C

C

C

C

C

C

C

C

C

C

C

C

C

C

C

C

C

C

C

C

C

C

C

C

C

subroutine rhic_magnet_instal1

implicit none

This routine was written for the purpose of installing magnets DX, D0, Q1 - Q5, and D5 into PISA/PISORP. The source of the information concerning the dimensions of the magnets was the RHIC Design Manual as released in October, 1994. In addition some dimensions were provided by Jack Sondericker.

The locations of the magnets are at best preliminary. The locations of the geometrical centers of the magnets were derived from so-called "strawman" diagrams that are undergoing development even now.

\section{Conventions}

All dimensions included below are expressed in centimeters. All lengths are half-lengths in order to conform to the conventions of GEANT. All angles are in degrees.

The path to common block gugeompar shown in the line below must be changed before use in other environments.

include 'gugeom.inc'

Additions made by C.F. Maguire during initial CVS implementation

Declare variables that are not associated with any particular magnet here.

real*4 angle1, angle2

integer*4 irot1, irot2, irot3, irot4, irot5, irot6, irot7

integer*4 irot8, irot9, irot10, irot11, irot12, irot13, irot14

integer*4 nmed, ivolu

Declare variables used in description of floor here.

real*4 x_coord_floor, y_coord_floor, z_coord_floor

real*4 floor par (3)

parameter ( $\mathrm{x}$ _coord_floor $=2.40 \mathrm{e}+02)$

parameter ( Y_coord_floor $=-1.9558 e+02)$

parameter ( z_coord_floor $=1.0550 e+04$ )

Declare variables to be used in description of walls here.

real*4 x_coord_wall, y_coord_wall, z_coord_wall

real*4 wall_par (3), x_coord_wall_2, z_coord_wal1_2

real*4 x_coord_wall_3, z_coord_wall_3

real*4 x_coord_wall_4, z_coord_wall_4

real*4 x_coord_wall_5, z_coord_wall_5

real*4 x_coord_wall_6, z_coord_wall_6

real*4 x_coord_wall_7, z_coord_wall_7

real*4 x_coord_wall_8, z_coord_wall_8

real*4 x_coord_wall_9, z_coord_wall_9

real*4 x_coord_wall_10, żcoord_walī_10

parameter $\left(y \_c o o r d \_w a l l=1.524 e+01\right)$ 
Declare variables used in description of ceiling here.

real*4 x_coord_ceiling, y_coord_ceiling, z_coord_ceiling

real*4 ceiling_par (3)

$\mathrm{C}$

C

$\mathrm{C}$

C

C

C

$\mathrm{C}$

C

C

$\mathrm{C}$

C

C

C

C

C

C

C

C

C

C

C

C

C

C

C

C

C

C

C

C

C

C

C

C

C

Note that we must not cover the experimental area with a ceiling!

parameter ( $x$ _coord_ceiling $=2.40 e+02$ )

parameter (y_coord_ceiling $=2.667 e+02$ )

parameter ( $z$ _coord_ceiling $=1.0550 e+04$ )

Declare variables used in description of DX here. Data

pertaining to DX were taken from pages 58-60 of the design

manual. Recall that DX is common to both arcs.

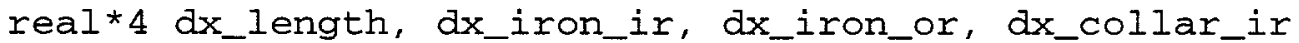

real*4 dx_collar_or, dx_x_coord, dx_y_coord, dx_z_coord

real*4 dx_tube_ir, dx_tube_or, dx_par (3)

real*4 dx_coil_ir, dx_coil_or

parameter $(\mathrm{dx}$ _iron_ir $=1.3335 \mathrm{e}+01, \mathrm{dx}$ _iron_or $=3.11 \mathrm{e}+01)$

parameter (dx_collar_ir $=1.0235 e+01, \overline{d x}$ _collar_or $=1.3335 e+01$ )

parameter (dx_coil_i $\bar{r}=9.0 e 0, d x \_c o i l \_o r=1.0 \overline{2} 35 e+01$ )

parameter (dx_tube_ir $=7.981 e 0, d_{x}$ _tube_or $\left.=8.70 e 0\right)$

parameter (dx_x_coord $\left.=0.0 e 0, d x \_y \_c o o r d ~=0.0 e 0\right)$

parameter $\left(d x \_z \_c o o r d=1.165 e+03, d x \_l\right.$ ength $\left.=1.85 e+02\right)$

Declare variables that are used in the description of Do here.

Data pertaining to DO were taken from pages 55 - 57 of the design manual. Additional information pertaining to the dimensions of beam tube was provided by Jack sondericker at BNL. There seems to be no agreement as to the magnetic length of DO. The RHIC design manual quotes 3.6 meters, but Jack Sondericker quotes 4.4 meters.

The Do magnets are actually slightly curved, but we will approximate them as being straight in this program.

real*4 do_length, do_iron_ir, do_iron_or, do_collar_ir

real*4 do_collar_or, do_x_coord, do_y_coord, do_z_coord

real*4 do_tube_ir, do_tube_or, do_par (3)

real*4 do_coil_ir, do_coil_or

real*4 theta_do_deg, $\bar{d}$ ist_ōdx_do

parameter (do_iron_ir $=6.970 \mathrm{e}$, do_iron_or $=1.55 \mathrm{e}+01$ )

parameter (do_collar_ir $=5.970 e 0, \overline{d o}$ collar_or $=6.970 e 0$ )

parameter (do_coil_ir $=5.0 e 0$, do_coil_or $=\overline{5} .970 e 0)$

parameter (do_tube_ir $=4.458 \mathrm{e}$, do_tube_or $=4.760 \mathrm{e}$ )

parameter (thêta_do_deg $=1.08064 \mathrm{e}$, dist_dx_do $=1.06516 \mathrm{e}+03$ )

parameter (do_lengt $\bar{h}=2.20 \mathrm{e}+02$, do_y_coord $=0.0 \mathrm{e} 0$ )

Declare variables that are used in the description of Q1, Q2, and Q3 here. These quads are identical except for their length. Variables that are common to all three quadrupoles are denoted by the prefix "qla", which stands for "large aperture quadrupole". The lengths of the quads are denoted by q1_length, q2_length, and q3ilength. The relevant pages in the RHIC design manual are $3 i \bar{i}, 3 i i i$, and $48-54$.

It is important to note that magnet $Q 2$ is really a corrector plus a quadrupole and that $\mathrm{Q} 3$ is really a corrector plus a quadrupole plus another quadrupole. The parameters q1_length, q2_length, and q3_length include the lengths of the associated corrector or correctors.

The inner diameter of the iron in these magnets varies between 
17.4 and $18.4 \mathrm{~cm}$. I have approximated the inner diameter by a constant value of $17.9 \mathrm{~cm}$. The spacer, which separates the coil from the yoke, also varies in thickness, and I have approximated the true thickness by a single value of $1.25 \mathrm{~cm}$.

real*4 q1_length, q2_length, q3_length

real*4 qla_iron_ir, qla_iron_or, qla_collar_ir

real*4 qla_collar_or, qla_theta_deg

real*4 q1_x_coord, q1_y_coord, q1_z_coord

real*4 q2_x_coord, q2_y_coord, q2_z_coord

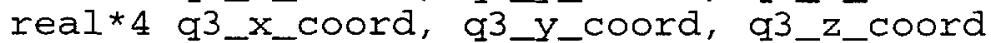

real*4 qla_tube_ir, qla_tube_or, qla_par (3)

real*4 qla_coil_ir, qla_coil_or

real*4 d0_q1_dist, q1_q2_dist, q2_q3_dist

parameter (qla_iron_ir $=8.950 e 0$, qla_iron_or $=1.7525 e+01$ )

parameter (qla_collar_ir $=7.700 \mathrm{e}$, qla_collar_or $=8.950 \mathrm{e}$ )

parameter (qla_coil_ir $=6.50 \mathrm{e}$, qla_coil_or $=7.700 \mathrm{e}$ )

parameter (qla_tube_ir $=5.636 e 0$, qla_tube_or $=6.033 \mathrm{e}$ )

parameter $(\mathrm{q} 1$ length $=9.5 e+01, q 2$ length $=2.20 \mathrm{e}+02)$

parameter (q3_length $=2.00 e+02, \mathrm{qla}$-theta_deg $=0.210536$ )

parameter $($ d0_q1_dist $=3.779 e+02, q \overline{1}$-q2_dist $=4.092 e+02)$

parameter (q2_q3_dist $=4.607 e+02, q 1$ y_coord $=0.0 e 0)$

parameter $\left(q^{2} \_y \_c o o r d=0.0 e 0, q 3 \_y_{-}\right.$coord $\left.=0.0 e 0\right)$

C

C

Declare variables that are used to describe Q4 and Q5 here. Q4 and Q5 are quadrupoles of standard aperture. These magnets are described on pages 27 - 36 of the RHIC design manual. Note that the dimensions of the yoke are the same as those of the arc dipoles. Numerical values for these parameters are shown on page 20 of the design manual.

Note also that both $\mathrm{Q} 4$ and $\mathrm{Q} 5$ are combinations of quadrupole, corrector, and trimmer. The lengths shown below include the lengths of all components.

real*4 q4_length, q5_length

real*4 qsa_iron_ir, qsa_iron_or, qsa_collar_ir

real*4 qsa_collar_or, qsa_theta_deg

real*4 q4_x_coord, q4_y_coord, q4_z_coord

real*4 q5_x_coord, q5_y_coord, q5_z_coord

real*4 qsa_tube_ir, qsa_tube_or, qsa_par (3)

real*4 qsa_coil_ir, qsa_coil_or

real*4 q3_q4_dist, q4_q5_dist

real*4 qsa_gap_ir, gsa_gap_or

parameter (qsa_iron_ir $=5.97 \mathrm{e}$, qsa_iron_or $=1.3335 \mathrm{e}+01$ )

parameter (qsa_coliar_ir $=5.00 e 0$, qsa_collar_or $=5.97 e 0$ )

parameter (qsa_coil_ir $=3.995 e 0$, qsa_coil_or $=5.00 \mathrm{e}$ )

parameter (qsa_gap_ir $=3.650$, qsa_gap_or $=3.995 e 0$ )

parameter (qsa_tube_ir $=3.455 e 0$, qsa_tube_or $=3.650 e 0$ )

parameter ( $q 4$ length $=2.050 \mathrm{e}+02$, q5_length $=1.70 \mathrm{e}+02$ )

parameter (qsa_theta_deg $=0.210536 \mathrm{e}$ )

parameter (q3_q4_dist $=4.04317 e+03, q 4$ q55_dist $=7.480 e+02$ )

parameter ( $\mathrm{q} 4$ _y_coord $=0.0 \mathrm{e} 0, \mathrm{q} 5$ _y_coord $=0.0 \mathrm{e}$ )

Declare variables that will be used to describe D5 below.

Note that there are differences between the D5 in the inner arc and D5 in the outer arc. For example the magnetic length of D5I is $6.92 \mathrm{~m}$ and the magnetic length of $\mathrm{D} 50$ is $8.71 \mathrm{~m}$.

Numerical values for the parameters for the parameters declared below were extracted from pages $14-24$ of the RHIC design manual.

real*4 d5_inner_length, d5_outer_length, d5_theta_deg real*4 d5_iron_ir, d5_iron_or, d5_collar_ir, d5_collar_or 
real*4 d5_out_x_coord, d5_out_y_coord, d5_out_z_coord

real*4 d5_in_x_coord, d5_in_y_coord, d5_in_z_coord

real*4 d5_tube_ir, d5_tube_or, d5_par ( $\overline{3}$ )

real*4 d5_coil_ir, d5_coil_or

real*4 q5_d5_inner_dist, q5_d5_outer_dist, d5_gap_ir, d5_gap_or

real*4 save_this_sine, save_this_cos

parameter (d5_iron_ir $=5.97 e 0, d 5$ _iron_or $=1.3335 \mathrm{e}+01$ )

parameter (d5_collar_ir $=5.00 e 0$, d5_collar_or $=5.97 e 0$ )

parameter (d5_coil_ir $=3.995 e 0$, d5_coil_or $=5.00 \mathrm{e} 0)$

parameter (d5_gap_ir $=3.650$, d5_gap_or $=3.995 \mathrm{e}$ )

parameter (d5_tube_ir $=3.455 e 0$, d5_tube_or $=3.650 \mathrm{e}$ )

parameter (d5_theta_deg $=0.210536 \mathrm{e}$ )

parameter (d5_inner_length $=3.46 \mathrm{e}+02$ )

parameter (d5_outer_length $=4.355 e+02$ )

parameter (q5_d5_inner_dist $=7.3980 e+02$ )

parameter (q5_d5_outer_dist $=7.4122 \mathrm{e}+02$ )

parameter ( $d \overline{5} \_$out_y_coord $=0.0 e 0, d 5 \_$in_y_coord $=0.0 e 0$ )

$\mathrm{C}$

$\mathrm{C}$

C

C

$\mathrm{C}$

C

$\mathrm{C}$

$\mathrm{C}$

C

C

C

C

C

$\mathrm{C}$

C

$\mathrm{C}$

C

Declare array for description of the lead absorber.

real*4 lead_abs_par (3)

Declare arrays for description of $Q 6$ below.

real*4 q6_in_x_coord, q6_in_y_coord, q6_in_z_coord real*4 g6_out_x_coord, q6_out_y_coord, q6_out_z_coord real*4 q6_length, d5_q6_in_dist, d5_q6_out_dist real*4 d5_d6_theta_deg

data d5_q6_in_dist / 7.3980e+02/

data d5_q6_out_dist / 7.4122e+02/

parameter $(\mathrm{q} 6$ _length $=1.70 \mathrm{e}+02, \mathrm{d5}$-d6_theta_deg $=1.84431)$

parameter $\left(q 6 \_\right.$in_y_coord $=0.0 e 0, q 6 \_$out_y_coord $=0.0 e 0$ )

Declare arrays for description of D6 below.

real*4 d6_in_x_coord, d6_in_y_coord, d6_in_z_coord

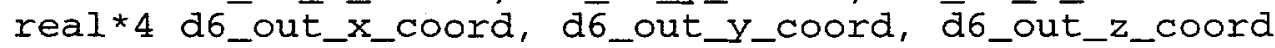
real*4 d6_length, d5_d6_in_dist, d5_d6_out_dist, d6_par(3)

data d5_d6_in_dist / $1.80496 e+03 /$ data d5_d6_out_dist / $1.80693 e+03 /$ parameter ( d6_length $=1.475 e+02)$ parameter (d6_in_y_coord $=0.0 e 0, d 6 \_$out_y_coord $=0.0 e 0$ )

Declare arrays for description of Q7 below.

real*4 q7_in_x_coord, q7_in_y_coord, q7_in_z_coord real*4 q7_out_x_coord, q7_out_y_coord, $\bar{q} 7$ out_z_coord real*4 q7_length, a6_q7_in_dist, d6_q7_out_dist real*4 d6_d8_theta_deg

data d6_q7_in_dist $/ 3.66166 e+02 /$

data d6_q7_out_dist / 3.66979e+02/

parameter ( q7 length $=1.25 e+02$ )

parameter (q7_in_y_coord $=0.0 e 0$, q7_out_y_coord $=0.0 e 0$ )

parameter (d6_d8_theta_deg $=2.54106 \mathrm{e} 0$ )

Declare arrays for $Q 8$ below.

real*4 q8_in_x_coord, q8_in_y_coord, q8_in_z_coord

real*4 q8_out_x_coord, q8_out_y_coord, q8_out_z_coord real*4 q8_length, d6_q8_in_dist, d6_q8_out_dist

data d6_q8_in_dist / 1.91847e+03/ 
data d6_q8_out_dist / $1.91928 e+03 /$

parameter ( q8_length $=1.30 e+02$ )

$\mathrm{C}$

parameter ( $q 8$ _in_y_coord $=0.0 e 0, q 8$ _out_y_coord $=0.0 e 0)$

Declare arrays for D8 below.

real*4 d8_in_x_coord, d8_in_y_coord, d8_in_z_coord

real*4 d8_out_x_coord, d8,out_y_coord, $\bar{d} 8$ _out_z_coord

C

real*4 d8_length, d6_d8_in_dist, d6_d8_out_dist, d8_par(3)

data d6_d8_in_dist $/ 2.61824 e+03 /$

data d6_d8_out_dist /2.62054e+03/

parameter ( d8_length $=4.725 e+02)$

parameter (d8_in_y_coord $=0.0 e 0$, d8_out_y_coord $=0.0 e 0$ )

$\mathrm{C}$

C

C

Declare arrays for $Q 9$ below.

real*4 q9_in_x_coord, q9 in_y_coord, q9'in_z_coord

real*4 q9_out_x_coord, q9 _out_y_coord, q9_out_z_coord

real*4 q9_length, d8_q9_in_dist, d8_q9_out_dist

C

real*4 d8_d9_theta_deg

data d8_q9_in_dist / 7.3966e+02/

data d8_q9_out_dist / 7.4141e+02/

parameter ( q9 length $=1.70 e+02$ )

parameter ( 99 -in_y_coord $=0.0 e 0$, g9_out_y_coord $=0.0 e 0$ )

parameter (d8_d9_theta_deg $=4.77124$ )

Declare arrays for $D 9$ below.

real*4 d9_in_x_coord, d9_in_y_coord, d9_in_z_coord

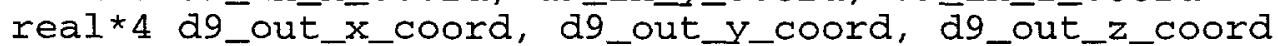

real*4 d9_length, d8_d9_in_dist, d8_d9_out_dist, d9_par(3)

data d8_d9_in_dist / 1.80482e+03/

data d8_d9_out_dist / 1.80712e+03/

parameter $(\mathrm{d} 9$ length $=1.475 \mathrm{e}+02)$

parameter (d9_in_y_coord $=0.0 e 0$, d9_out_y_coord $=0.0 e 0$ )

Addition for the use of the PISA geometry namelist control file phnx.par

Parameters for RHIC_MAGNET_INSTALL will be in the rhic_mag segment

integer nmed_g10 /24/ ! tracking medium number for G10

integer nmed_dx $/ 41 /$ ! tracking medium for DX magnet

integer nmed_cu /15/ ! tracking medium for Copper

namelist /rhic_mag/ nmed_g10, nmed_dx, nmed_cu

Begin execution

Read the geometery file segment rhic_mag

open (unit=15, file='phnx . par', status=' OLD', err=997)

read $(15, \mathrm{nml}=$ rhic_mag, err $=999)$

close (unit=15)

Install floor.

nmed $=442$

floor_par $(1)=6.50 e+02$

floor_par $(2)=4.318 e+01$

floor par $(3)=9.450 e+03$

call gsvolu ('FLOR', 'BOX ', nmed, floor_par, 3, ivolu)

call gsatt ('FLOR', 'COLO', 7)

call gspos ('FLOR', 1, 'HALL', x_coord_floor, Y_coord_floor, 
C

C

C

C

$\mathrm{C}$

C

C

C

$\mathrm{C}$

C

C

C

call gspos ('FLOR', 2, 'HALL', x_coord_floor, Y_coord_floor, $-z$ zcoord_floor, 0 , 'ONLY')

Install wall, section 1 .

$x \_c o o r d \_w a l 1=3.0480 e+02$

z_coord_wall $=5.60 e+03$

wall_par $(1)=5.334 \mathrm{e}+01$

wall par $(2)=1.67 e+02$

wal1_par $(3)=4.40 \mathrm{e}+03$

call gsvolu ('WALl', 'BOX', nmed, wall_par, 3, ivolu)

call gsatt ('WAL1', 'COLO', 1)

call gspos ('WAL1', 1, 'HALL', x_coord_wal1, Y_coord_wall,

\$ z_coord_wall, 0 , 'ONLY')

call gspos ('WALl', 2, 'HALI', - x_coord_wall, y_coord_wall, $\$$ $z$ coord wal1, 0 , 'ONLY')

call gspos ('WAL1', 3, 'HALL', - x_coord_wall, y_coord_wall,

$\$ \quad-z \_c o o r d \_w a l l, 0$, 'ONLY')

call gspos ('WAL1', 4, 'HALL', x_coord_wal1, Y_coord_wall, $\$$ $-z$ _coord_wal1, 0 , 'ONLY')

Install wall, section 2 .

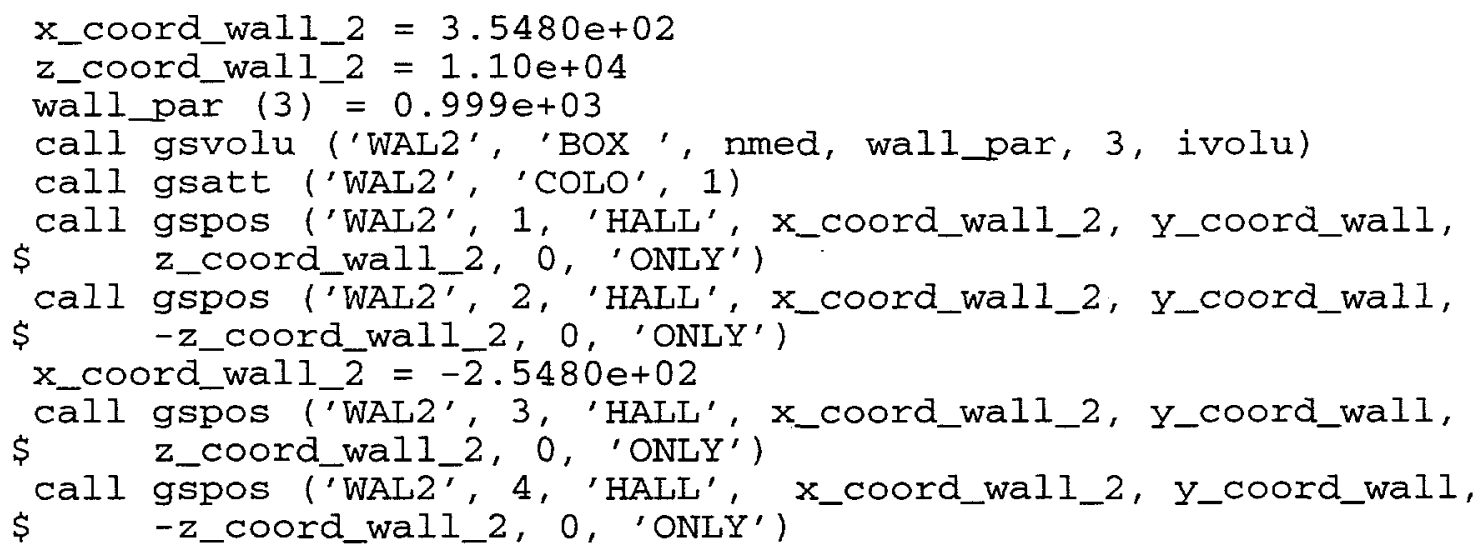

Install wall, section 3 .

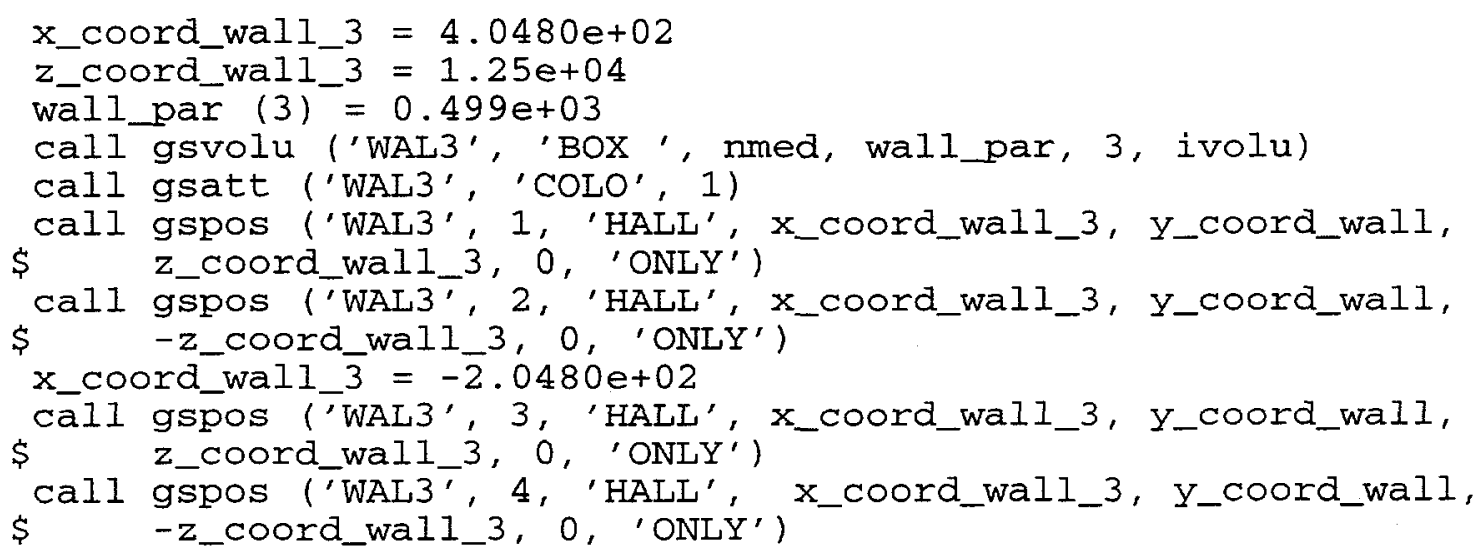


cal1 gspos ('WAL4', 3, 'HALI', x_coord_wall_4, y_coord_wall,

call gspos ('WAL4', 4,' 'HALL', x_coord_wal1_4, y_coord_wal1, $\$$ -z_coord_wal1_4, 0 , 'ONLY')

Install wall, section 5 .

$x \_$coord_wal1_5 $=5.0480 e+02$

z_coord_wal1_5 $=1.45 e+04$

call gsvolu ('WAL5','Box', nmed, wall_par, 3, ivolu)

call gsatt ('WAL5', 'COLO', 1)

call gspos ('WAL5', 1, 'HALL', x_coord_wal1_5, y_coord_wall,

call gspos ('WAL5', 2, 'HALL', x_coord_wal1_5, y_coord_wall,

$\$ \quad-z_{-}$coord_wall_5, 0 , 'ONLY')

$x \_$coord_wall_ $\overline{5}=-\overline{1} .0480 e+02$

call gspos ('WAL5', 3, 'HALL', x_coord_wall_5, y_coord_wall,

\$ z_coord_wall_5, 0 , 'ONLY')

cal1 gspos ('WAL5', 4, 'HALL', x_coord_wall_5, Y_coord_wall, $\$$ $-z \_$coord_wall_5, 0 , 'ONLY')

Install wall, section 6 .

x_coord_wal1_6 $=5.5480 e+02$

$z_{-}$coord_wall_6 $=1.55 \mathrm{e}+04$

call gsvolu ('WAL6', 'BOX ', nmed, wall_par, 3, ivolu)

call gsatt ('WAL6',' 'COLO', 1)

call gspos ('WAL6', 1, 'HALL', x_coord_wall_6, y_coord_wall, $\$ \quad$ z_coord_wall_6, 0, 'ONLY')

call gspos ('WAL6', 2, 'HALL', x_coord_wall_6, y_coord_wall, $\$ \quad-z$ _coord_wal1_6, 0, 'ONLY')

x_coord_wal1_ $\overline{6}=-\overline{5} .480 e+01$

call gspos ('WAL6', 3, 'HALL', x_coord_wall_6, y_coord_wall, $\$$ z_coord_wall_6, 0 , 'ONLY')

call gspos ('WAL6', 4,'HALL', x_coord_wall_6, y_coord_wall,

$\$ \quad-z \_c o o r d \_w a l 1 \_6,0$, 'ONLY')

Install wall, section 7 .

x_coord_wall_7 $=6.0480 e+02$

z_coord_wall_7 $=1.65 e+04$

call gsvolu ('WAL7', 'BOX ', nmed, wall_par, 3, ivolu)

call gsatt ('WAL7', 'COLO', 1)

call gspos ('WAL7', 1, 'HALL', x_coord_wall_7, y_coord_wall, $\$$

call gspos ('WAL7', 2,' 'HALL', x_coord_wall_7, y_coord_wall, $\$ \quad-z+c o o r d \_w a l l \_7,0$, 'ONLY' $^{\prime}$

$\mathrm{x}$ _coord_wall_ $\overline{7}=-\overline{0} .480 \mathrm{e}+01$

call gspos ('WAL7', 3, 'HALL', x_coord_wall_7, y_coord_wall,

$\$ \quad$ z_coord_wall_7, 0 , 'ONLY')

call gspos ('WAL7', 4, 'HALL', x_coord_wall_7, y_coord_wall,

$\$ \quad-z \_c o o r d \_w a l 1 \_7,0$, 'ONLY' $^{\prime}$

Install wall, section 8 .

x_coord_wall_8 $=6.5480 e+02$

z_coord_wall_8 $=1.75 \mathrm{e}+04$

call gsvolu ('WAL8', 'BOX ', nmed, wall_par, 3, ivolu)

call gsatt ('WAL8', 'COLO', 1)

call gspos ('WAL8', 1, 'HALL', x_coord_wall_8, y_coord_wall,

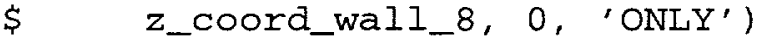

call gspos ('WAL8', 2, 'HALL', x_coord_wall_8, y_coord_wall,

$\$ \quad-z+c o o r d$ wall_8, 0 , 'ONLY')

x_coord_wall_ $8=4.520 e+01$

call gspos ('WAL8', 3, 'HALL', x_coord_wall_8, y_coord_wall, $\$$ z_coord_wall_8, 0, 'ONLY') 
C

call gspos ('WAL8', 4, 'HALL', x_coord_wall_8, y_coord_wall, -z_coord_wall_8, 0 , 'ONLY')

Install wall, section 9 .

x_coord_wa11_9 $=7.0480 e+02$

z_coord_wall_g $=1.85 \mathrm{e}+04$

call gsvolu ('WAL9', 'BOX', nmed, wall_par, 3, ivolu)

call gsatt ('WAL9', 'COLO', 1)

call gspos ('WAL9', 1, 'HALL', x_coord_wall_9, y_coord_wall, $\$$

cal1 gspos ('WAL9', 2, 'HALL', x_coord_wal1_9, y_coord_wal1, $\$$ x_coord_wal1_ $\overline{9}=9.520 e+01$

call gspos ('WAL9', 3, 'HALL', x_coord_wall_9, y_coord_wal1, $\$$

$\$ \quad-z \_$coord_wal1_9, 0 , 'ONLY')

Install wal1, section 10 .

x_coord_wal1_10 $=7.5480 e+02$

z_coord_wa11_10 = $1.95 e+04$

call gsvolu ('WALA', 'BOX ', nmed, wallpar, 3, ivolu)

call gsatt ('WALA', 'COLO', 1)

call gspos ('WALA', 1, 'HALL', x_coord_wal1_10, Y_coord_wall,

$\$$

call gspos ('WALA', 2, 'HALL', x_coord_wal1_10, y_coord_wal1,

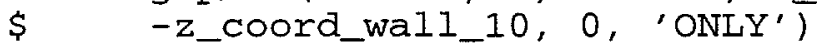

$x \_$coord_wal1_10 $=\overline{1} .4520 e+02$

call gspos ('WALA', 3, 'HALL', x_coord_wall_10, y_coord_wall, $\$ \quad$ z_coord_wall_10, 0, 'ONLY')

call gspos ('WALA', 4, 'HALL', x_coord_wal1_10, Y_coord_wall, $\$$ $-z$ _coord_wal1_10, 0 , 'ONLY')

Begin installation of ceiling.

ceiling par $(1)=6.50 e+02$

ceiling_par $(2)=8.382 \mathrm{e}+01$

ceiling_par $(3)=9.45 e+03$

call gsvolu ('CEIL', 'BOX', nmed, ceiling_par, 3, ivolu)

call gspos ('CEIL', 1, 'HALL', x_coord_ceiling, y_coord_ceiling, \$ $\quad$ __coord_ceiling, 0 , 'ONLY')

call gspos ('CEIL', 2, 'HALL', x_coord_ceiling, y_coord_ceiling, $\$$ - z_coord_ceiling, 0 , 'ONLY')

Begin installation of magnets. Start with DX.

$d x \_$par $(1)=d x \_i r o n \_i r$

$d x \_$par $(2)=d x$ _iron_or

$d x \_$par $(3)=d x \_$length

nmed = nmed_dx

call gsvolu ('DXFE', 'TUBE', nmed, dx_par, 3, ivolu)

call gsatt ('DXFE', 'COLO', 6)

call gspos ('DXFE', 1, 'HALL', dx_x_coord, dx_y_coord, $\$$ dx_z_coord, 0, 'ONLY')

Unlike all other magnets, the collar of DX is made of stainless steel. Eventually we must define stainless steel, but for the moment we will, regard the collar as being material 900 .

$d x \operatorname{par}(1)=d x$ collar ir

$d x \_p a r(2)=d x \_c o l l a r \_o r$

nmed $=900$

call gsvolu ('DXCL', 'TUBE', nmed, dx_par, 3, ivolu) 
call gsatt ('DXCL', 'COLO', 6)

Don't clutter up the graphics output by displaying anything but the iron.

call gsatt ('DXCL', 'SEEN', 0 )

call gspos ('DXCL', 1, 'DXFE', $0.0 e 0,0.0 e 0,0.0 e 0,0$, 'ONLY')

The coil consists of superconducting Niobium Titanium wire embedded in a Copper matrix. Because the $\mathrm{Cu}$ constitutes approximately $64 \%$ of the coil, we will temporarily regard the coil as being cu.

$d x \_p a r(1)=d x \_c o i l \_i r$

dx_par $(2)=d x \_c o i l \_o r$

nmed = nmed_cu

call gsvolu ('DXCO', 'TUBE', nmed, dx_par, 3, ivolu)

call gsatt ('DXCO', 'COLO', 6)

call gsatt ('DXCO', 'SEEN', 0 )

call gspos ('DXCO', 1, 'DXCL', $0.0 e 0,0.0 e 0,0.0 e 0,0$, 'ONLY') $^{\circ}$

The geometry of the beam tube is complicated. In fact there isn't even a single tube in DX. The engineers thought it a bad idea for a tube at 4 Kelvin to be exposed to room temperature, so they buried the tube in a second tube and stuffed the space between the two tubes with superinsulation. According to Jack Sondericker, the dimensions of the two tubes are as follows:

$O D$ of outer tube $=17.40 \mathrm{~cm} \quad O D$ of inner tube $=14.29 \mathrm{~cm}$

wall thickness $=0.559 \mathrm{~cm} \quad$ wall thickness $=0.160 \mathrm{~cm}$

We will use just one tube and set the thickness of the tube to the sum of the thicknesses shown above.

$d x \_p a r(1)=d x \_t u b e \_i r$

$d x \_$par $(2)=d x \_t u b e_{\text {or }}$

nmed $=900$

call gsvolu ('DXTU', 'TUBE', nmed, dx_par, 3, ivolu)

call gsatt ('DXTU', 'COLO', 6)

call gsatt ('DXTU', 'SEEN', 0 )

call gspos ('DXTU', 1, 'DXCO', $0.0 e 0,0.0 e 0,0.0 e 0,0$, 'ONLY')

Install the vacuum inside the tube.

$d x \_$par $(1)=0.0 e 0$

$d x \_$par $(2)=d x \_t u b e \_i r$

nmed $=16$

call gsvolu ('DXVA', 'TUBE', nmed, dx_par, 3, ivolu)

call gsatt ('DXTU',' 'SEEN', 0 )

call gspos ('DXVA', 1, 'DXTU', 0.0e0, $0.0 e 0,0.0 e 0,0$, 'ONLY')

Because DX is common to both arcs on the same side of the interaction point, it is only necessary that we create a second copy on the other side of the interaction point.

call gspos ('DXFE',2, 'HALL', dx_x_coord, dx_y_coord,
-dx_z_coord, 0 ,' 'ONLY')

First, install the iron of DO. Note that the coordinates of the geometrical center are calculated with respect to the coordinates of the geometrical center of DX.

Note that the functions sind and cosd are non-standard functions that may not be defined on other machines.

d0_x_coord $=$ - dist_dx_do * sind (theta_do_deg)

do_z_coord $=d x \_z \_c o o r d+d i s t \_d x \_d 0 *$ cosd $($ theta_do_deg) 
do_par $(1)=$ do_iron_ir

do_par $(2)=$ do_iron_or

do_par $(3)=$ do_length

nmed = nmed_dx

call gsvolu ('DOFE', 'TUBE', nmed, do_par, 3, ivolu)

call gsatt ('DOFE',' 'COLO', 6)

The coordinate system of $D O$ is rotated with respect to the master coordinate sytem, so define the appropriate rotation matrix. Note that the following rotation describes the magnet in the outside arc.

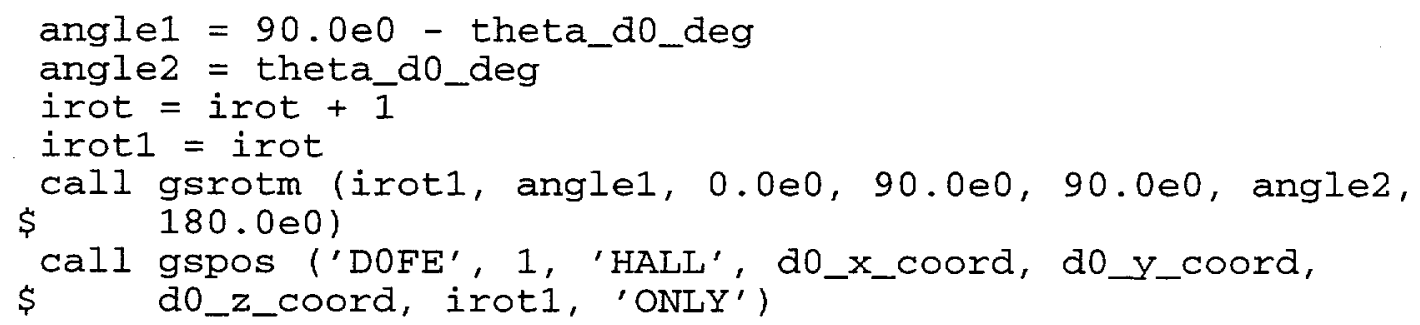

The collar of DO is made from RX630 (Phenolic) molding. We regard phenoic as being G-10 at the moment.

d0_par $(1)=$ d0_collar_ir

do_par $(2)=$ do_collar_or

nmed = nmed_g1 $\overline{0}$

call gsvolu ('DOCL', 'TUBE', nmed, do_par, 3, ivolu)

call gsatt ('DOCL', 'COLO', 6)

call gsatt ('DOCL', 'SEEN', 0 )

call gspos ('DOCL', 1, 'DOFE', 0.0e0, 0.0e0, 0.0e0, 0, 'ONLY')

Now install the coil to Do.

do_par $(1)=$ do_coil_ir

do_par $(2)=$ do_coil_or

nmed = nmed cu

call gsvolu ('DOCO', 'TUBE', nmed, do_par, 3, ivolu)

call gsatt ('DOCO', 'COLO', 6)

call gsatt ('DOCO', 'SEEN', 0)

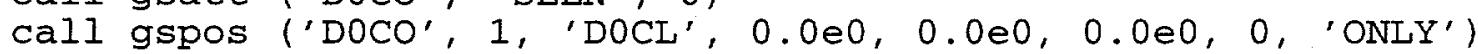

C

C

C

C

$\mathrm{C}$

$\mathrm{C}$

C

$\mathrm{C}$

C

C

$\mathrm{C}$

Now install the beam tube. The dimensions of the tube were provided by Jack Sondericker, and there seems to be some inconsistency between these numbers and the RHIC design manual.

do_par $(1)=$ do_tube_ir

do_par $(2)=$ do_tube_or

nmed $=900$

call gsvolu ('DOTU', 'TUBE', nmed, do_par, 3, ivolu)

call gsatt ('DOTU', 'COLO', 6)

call gsatt ('DOTU', 'SEEN', 0)

call gspos ('DOTU', 1, 'DOCO', $0.0 \mathrm{e} 0,0.0 \mathrm{e} 0,0.0 \mathrm{e} 0,0$, 'ONLY')

Install the vacuum inside the tube.

do_par $(1)=0.0 e 0$

do_par $(2)=$ do_tube_ir

nmed $=16$

call gsvolu ('DOVA', 'TUBE', nmed, do_par, 3, ivolu)

call gsatt ('DOTU', 'SEEN', 0)

call gspos ('DOVA', 1, 'DOTU', 0.0e0, $0.0 e 0,0.0 e 0,0$, 'ONLY')

There are two DO magnets on each side of the interaction point, making a total of four magnets in all. Note the necessity for 
additional rotation matrices.

First, install the second magnet on the same side of the interaction point.

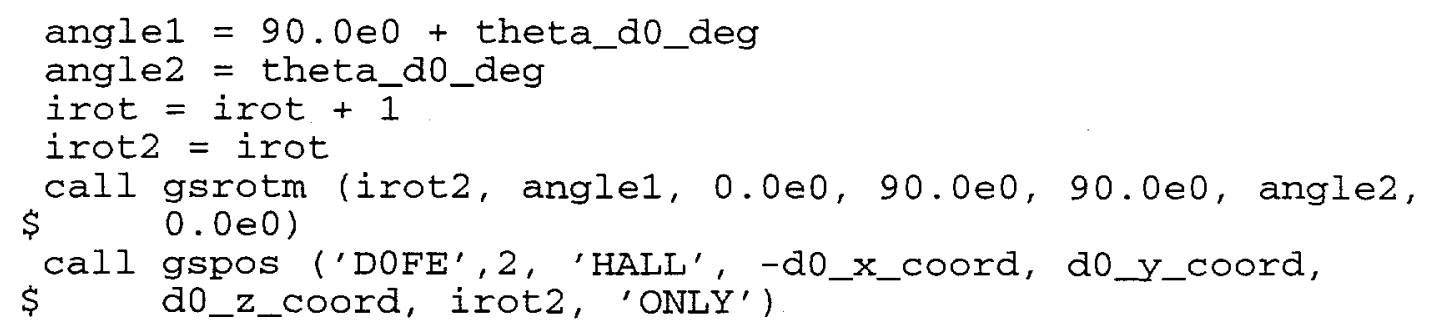

Now install the two magnets on the other side of the interaction point.

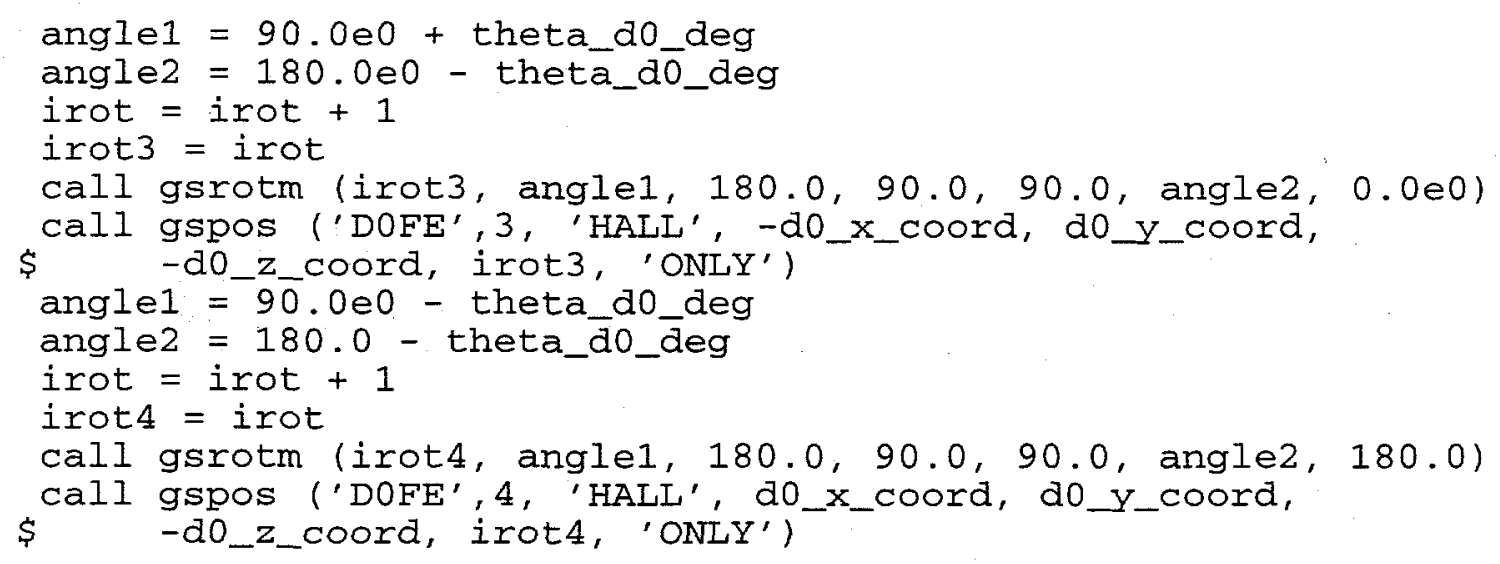

$\mathrm{C}$

Install Q1. First, calculate the coordinates of the geometrical center of the magnet.

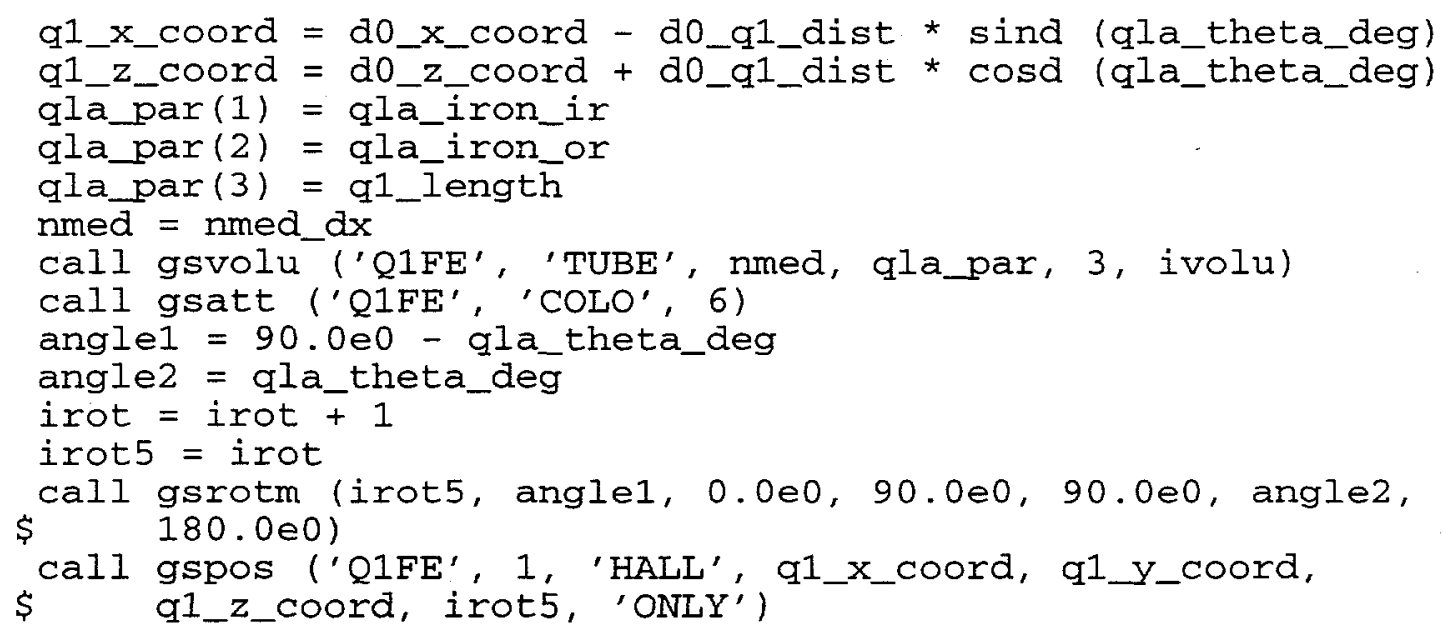

The collar of the quads is made from Rx630 molding. We regard RX630 as being $G-10$ at the moment.

qla_par $(1)=$ qla_collar_ir

qla_par $(2)=$ qla_collar_or

nme $\bar{d}=$ nmed_g10

call gsvolu ('Q1CL', 'TUBE', nmed, qla_par, 3, ivolu)

call gsatt ('Q1CL', 'COLO', 6)

call gsatt ('Q1CL', 'SEEN', 0 )

call gspos ('Q1CL', 1, 'Q1FE', $0.0 e 0,0.0 e 0,0.0 e 0,0$, 'ONLY')

Now install the coil to $Q 1$.

qla_par $(1)=$ qla_coil_ir 
qla_par $(2)=q l a \_c o i l \_o r$

nmed = nmed_cu

call gsvolu ('Q1CO', 'TUBE', nmed, qla_par, 3, ivolu)

call gsatt ('Q1CO', 'COLO', 6)

call gsatt ('Q1CO', 'SEEN', 0 )

call gspos ('Q1CO', 1, 'Q1CL', 0.0e0, $0.0 \mathrm{e} 0,0.0 \mathrm{e} 0,0$, 'ONLY')

Now install the beam tube. The dimensions of the tube were provided by Jack Sondericker, and there seems to be a slight inconsistency between these numbers and the RHIC design manual.

qla_par $(1)=$ qla_tube_ir

qla_par $(2)=q l a \_t u b e \_o r$

nmed $=900$

call gsvolu ('Q1TU', 'TUBE', nmed, qla_par, 3, ivolu)

call gsatt ('Q1TU', 'COLO', 6)

call gsatt ('Q1TU', 'SEEN', 0)

call gspos ('Q1TU', 1, 'Q1CO', $0.0 e 0,0.0 e 0,0.0 e 0,0$, 'ONLY')

C

C

$\mathrm{C}$

C

$\mathrm{C}$

C

C

$\mathrm{C}$

C

C

C

Install the vacuum inside the tube.

qla_par $(1)=0.0 e 0$

qla_par $(2)=$ qla_tube_ir

nmed $=16$

call gsvolu ('Q1VA', 'TUBE', nmed, qla_par, 3, ivolu)

call gsatt ('Q1TU', 'SEEN', 0 )

call gspos ('Q1VA', 1, 'Q1TU', $0.0 e 0,0.0 e 0,0.0 e 0,0$, 'ONLY')

There are two Q1 magnets on each side of the interaction point making a total of four magnets in all. Note the necessity for rotation matrices.

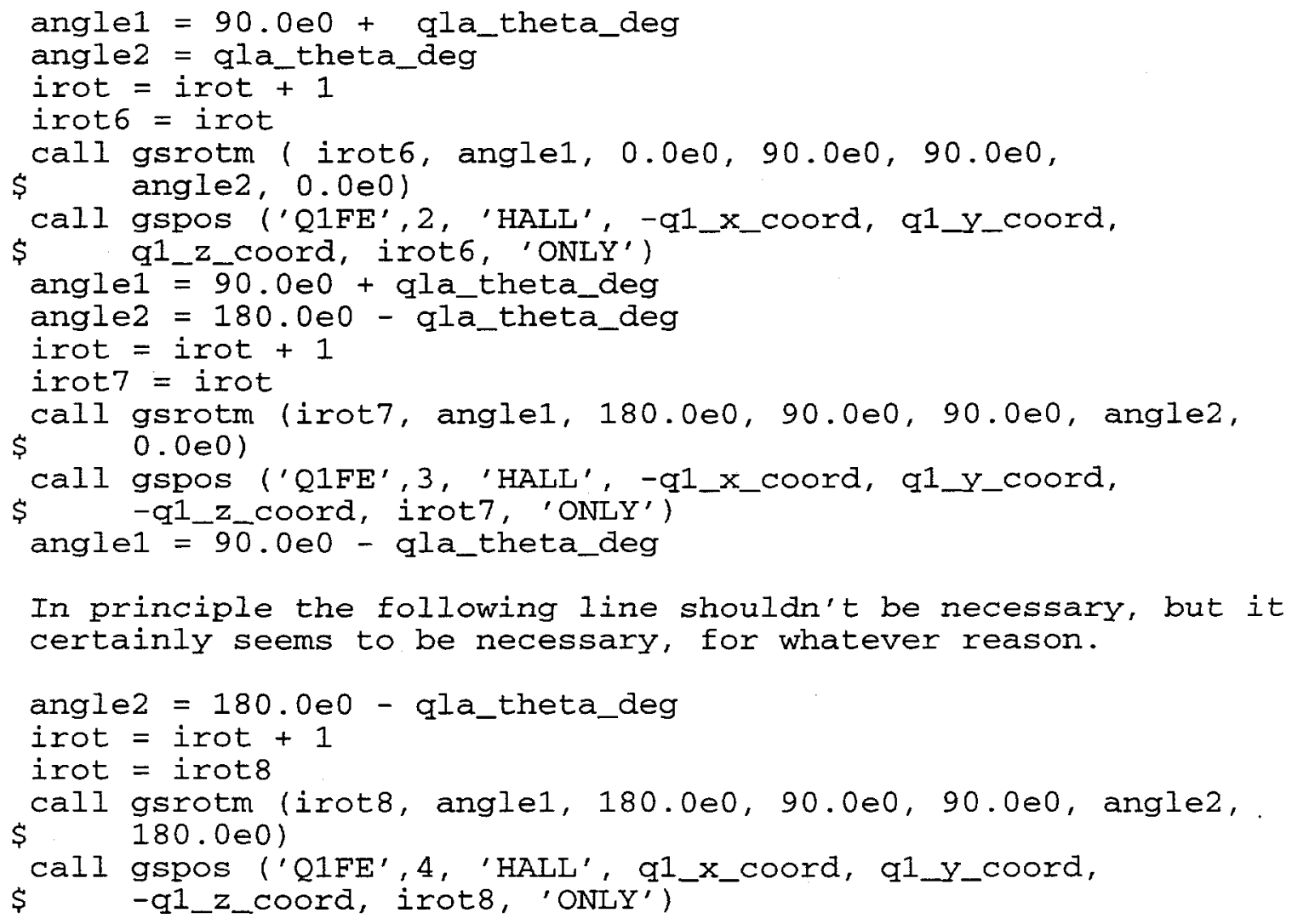

Install Q2. First, calculate the coordinates of the geometrical center of the magnet.

$q 2 \_x \_c o o r d=q 1$ x_coord $-q 1$ q2_dist * sind (qla_theta_deg)

$q^{2} z_{2} \_c o o r d=q 1{ }^{2}$ _coord $+q 1$ q 2 _dist $*$ cosd (qla_theta_deg) 
qla_par $(1)=q l a \_i r o n \_i r$

qla_par $(2)=$ qla_iron_or

qla_par $(3)=$ q2_length

nmed = nmed_dx

call gsvolu ('Q2FE', 'TUBE', nmed, qla_par, 3, ivolu)

call gsatt ('O2FE', 'COLO', 6)

$\mathrm{C}$

call gspos ('Q2FE', 1, 'HALL', q2_x_coord, q2_Y_coord,

C

Install the collar to Q2.

qla_par $(1)=$ qla_collar_ir

qla_par $(2)=$ qla_collar_or

nmed = nmed_g10

call gsvolu ('Q2CL', 'TUBE', nmed, qla_par, 3, ivolu)

call gsatt ('Q2CL', 'COLO', 6)

call gsatt ('Q2CL', 'SEEN', 0 )

call gspos ('Q2CL', 1, 'Q2FE', $0.0 e 0,0.0 e 0,0.0 e 0,0$, 'ONLY')

$\mathrm{C}$

C

c

$\mathrm{C}$

$\mathrm{C}$

$\mathrm{C}$

C

$\mathrm{C}$

Now install the coil to 22 .

qla_par(1) = qla_coil_ir

qla_par $(2)=$ qla_coil_or

nmed = nmed_cu

call gsvolu ('Q2CO', 'TUBE', nmed, qla_par, 3, ivolu)

call gsatt ('Q2CO', 'COLO', 6)

call gsatt ('Q2CO', 'SEEN', 0 )

call gspos ('Q2CO', 1, 'Q2CL', $0.0 e 0,0.0 e 0,0.0 e 0,0$, 'ONLY')

Now install the beam tube.

qla_par(1) = qla_tube_ir

qla_par $(2)$ = qla_tube_or

nmed $=900$

call gsvolu ('Q2TU', 'TUBE', nmed, qla_par, 3, ivolu)

call gsatt ('Q2TU', 'COLO', 6)

call gsatt ('Q2TU', 'SEEN', 0)

call gspos ('Q2TU', 1, 'Q2CO', $0.0 e 0,0.0 e 0,0.0 e 0,0$, 'ONLY')

Install the vacuum inside the tube.

qla_par $(1)=0.0 e 0$

qla_par $(2)$ = qla_tube_ir

nmed $=16$

call gsvolu ('Q2VA', 'TUBE', nmed, qla_par, 3, ivolu)

call gsatt ('Q2TU', 'SEEN', 0$)$

call gspos ('Q2VA', 1, 'Q2TU', $0.0 e 0,0.0 e 0,0.0 e 0,0$, 'ONLY')

Install the other Q2 magnets.

call gspos ('Q2FE',2, 'HALL', -q2_x_coord, q2_y_coord,

$\$$ q2_z_coord, irot6, 'ONLY')

call gspos ('Q2FE', 3, 'HALI', -q2_x_coord, q2_y_coord, $\$$ -q2_z_coord, irot7, 'ONLY')

call gspos ('Q2FE',4, 'HALL', q2_x_coord, q2_y_coord, $\$$ -q2_z_coord, irot8, 'ONLY')

C

$\mathrm{C}$

Install Q3. First, calculate the coordinates of the geometrical center of the magnet.

$q^{3} \_x \_c o o r d=q 2 \_x \_c o o r d-q 2 \_q 3$ dist $*$ sind (qla_theta_deg) q3_z_coord $=q 2{ }^{3} z_{-}$coord $+q^{2}$-q3 3 dist $*$ cosd (qla_theta_deg)

qla_par $(1)=q l a \_i r o n \_i r$

qla_par $(2)=$ qla_iron_or

qla_par $(3)=$ q3_lengt $\bar{h}$

nmed $=$ nmed_dx 
call gsvolu ('Q3FE', 'TUBE', nmed, qla_par, 3, ivolu)

call gsatt ('Q3FE',' 'COLO', 6)

call gspos ('Q3FE', 1, 'HALL', q3_x_coord, q3_y_coord,

$\mathrm{C}$

C

$\mathrm{C}$

$\mathrm{C}$

C

C

$\mathrm{C}$

C

$\mathrm{C}$

Install the collar inside $Q 3$.

qla_par $(1)=$ qla_collar_ir

qla_par $(2)=$ qla_collar_or

nmed = nmed_g10

call gsvolu ('Q3CL', 'TUBE', nmed, qla_par, 3, ivolu)

call gsatt ('Q3CL', 'COLO', 6)

call gsatt ('Q3CL', 'SEEN', 0 )

cal1 gspos ('Q3CL', 1, 'Q3FE', $0.0 e 0,0.0 e 0,0.0 e 0,0, ' O N L Y '$ )

Now instal1 the coil inside $Q 3$.

qla_par $(1)=q l a \_c o i l \_i r$

qla_par $(2)=$ qla_coil_or

nmed $=$ nmed_cu

call gsvolu ('Q3Co', 'TUBE', nmed, qla_par, 3, ivolu)

call gsatt ('Q3 $\mathrm{CO},$, 'COLO', 6)

call gsatt ('Q3CO', 'SEEN', 0 )

call gspos ('Q3CO', 1, 'Q3CL', $0.0 e 0,0.0 e 0,0.0 e 0,0$, 'ONLY')

Now install the beam tube inside $Q 3$.

qla_par $(1)=$ qla_tube_ir

qla_par $(2)=$ qla_tube_or

nmed $=900$

call gsvolu ('Q3TU', 'TUBE', nmed, qla_par, 3, ivolu)

call gsatt ('Q3TU', 'COLO', 6)

call gsatt ('Q3TU', 'SEEN', 0 )

call gspos ('Q3TU', 1, 'Q3CO', $0.0 \mathrm{Ce}, 0.0 \mathrm{~T} 0,0.0 \mathrm{e}, 0, \mathrm{O}, \mathrm{ONLY}$ ')

Install the vacuum inside the tube.

qla_par $(1)=0.0 e 0$

qla_par $(2)=$ qla_tube_ir

nmed $=16$

call gsvolu ('Q3VA', 'TUBE', nmed, qla_par, 3, ivolu)

cal1 gsatt ('Q3TU', 'SEEN', 0 )

call gspos ('Q3VA', 1, 'Q3TU', $0.0 e 0,0.0 e 0,0.0 e 0,0$, 'ONLY')

Install the other Q3 magnets.

call gspos ('Q3FE',2, 'HALL', -q3_x_coord, q3_y_coord, $\$$ q3_z_coord, irot6, 'ONLY')

call gspos ('Q3FE', 3, 'HALL', -q3_x_coord, q3_y_coord, $\$-q 3 \ldots z$ coord, irot7, 'ONLY')

cal1 gspos ('Q3FE', 4, 'HALL', q33x_coord, q3_y_coord, $\$ \quad-q^{3} z_{2}$ coord, irot8, 'ONLY')

The code that follows was inserted in order to study the effect of an absorber upon produced particles that originate in region 3 . The coordinates of the center of the slab are chosen to coincide with the position of the valve that separates region 2 from region 3. The end of this special insertion is marked with a comment line that contains the special characters $* \star \star \star \star * *$

$$
\begin{aligned}
& \text { nmed }=9 \\
& \text { lead_abs_par }(1)=12.0 e 0 \\
& \text { lead_abs_par }(2)=8.00 e+02 \\
& \text { lead_abs_par ( } 3)=3.0 e 0 \\
& \text { call gsvolu ('ABPB', 'TUBE', nmed, lead_abs_par, 3, ivolu) }
\end{aligned}
$$




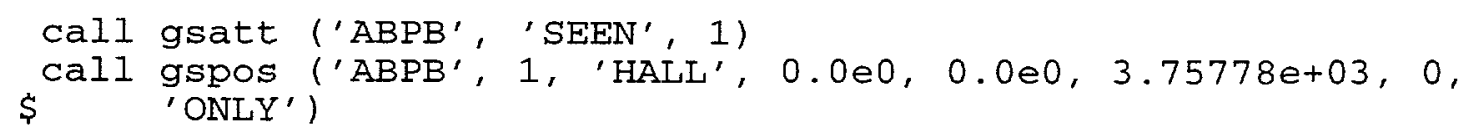

Install Q4. First, calculate the coordinates of the geometrical center of the magnet.

q4_x_coord $=q^{3}$ - $x$ _coord $-q^{3} \_q 4$-dist $*$ sind (qsa_theta_deg)

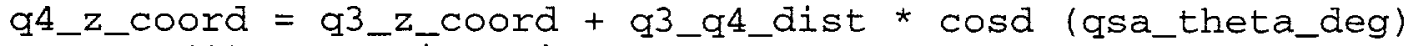

qsa_par(1) = qsa_iron_ir

qsa_par (2) = qsa_iron_or

qsa_par $(3)=q 4$ length

nmed = nmed_dx

call gsvolu ('Q4FE', 'TUBE', nmed, gsa_par, 3, ivolu)

call gsatt ('Q4FE', 'COLO', 6)

$\mathrm{C}$

call gspos ('Q4FE', 1, 'HALL', q4_x_coord, q4_y_coord, $\$$

$$
\text { q4_z_coord, irot5, 'ONLY') }
$$

Install the collar inside Q4.

qsa_par $(1)=$ qsa_collar_ir

qsa_par (2) = qsa_collar_or

nmed $=$ nmed_g10

call gsvolu ('Q4CL', 'TUBE', nmed, qsa_par, 3, ivolu)

call gsatt ('Q4CL',' 'COLO', 6)

call gsatt ('Q4CL', 'SEEN', 0 )

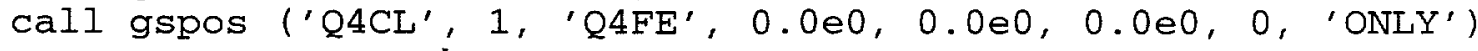

Now install the coil inside $\mathrm{Q} 4$.

qsa_par(1) = qsa_coil_ir

qsa_par $(2)$ = qsa_coil_or

nmed $=$ nmed_cu

call gsvolu ('Q4CO', 'TUBE', nmed, qsa_par, 3, ivolu)

call gsatt ('Q4CO', 'COLO', 6)

call gsatt ('Q4CO', 'SEEN', 0$)$

call gspos ('Q4CO', 1, 'Q4CL', $0.0 e 0,0.0 e 0,0.0 e 0,0$, 'ONLY')

$\mathrm{C}$

$\mathrm{C}$

- $\mathrm{C}$

Install the gap between the beam tube and the coil.

qsa_par (1) = qsa_gap_ir

qsa_par $(2)=$ qsa_gap_or

nmed $=16$

call gsvolu ('Q4GA', 'TUBE', nmed, qsa_par, 3, ivolu)

call gsatt ('Q4GA', 'COLO', 6)

call gsatt ('Q4GA', 'SEEN', 0$)$

call gspos ('Q4GA', 1, 'Q4CO', $0.0 e 0,0.0 e 0,0.0 e 0,0$, 'ONLY')

Now install the beam tube inside $Q 4$.

gsa_par(1) = qsa_tube_ir

qsa_par $(2)$ = qsa_tube_or

nmed $=900$

call gsvolu ('Q4TU', 'TUBE', nmed, qsa_par, 3, ivolu)

call gsatt ('Q4TU', 'COLO', 6)

call gsatt ('Q4TU', 'SEEN', 0 )

call gspos ('Q4TU', 1, 'Q4GA', $0.0 e 0,0.0 e 0,0.0 e 0,0$, 'ONLY')

Install the vacuum inside the tube.

qsa_par $(1)=0.0 \mathrm{e} 0$

qsa_par $(2)=$ gsa_tube_ir

nmed $=16$

call gsvolu ('Q4VA', 'TUBE', nmed, qsa_par, 3, ivolu)

call gsatt ('Q4TU', 'SEEN', 0)

call gspos ('Q4VA', 1, 'Q4TU', $0.0 e 0,0.0 e 0,0.0 e 0,0, \mathrm{VN}^{\mathrm{T}} \mathrm{ONY}$ ') 
Install the other $Q 4$ magnets.

call gspos ('Q4FE', 2, 'HALL', -q4_x_coord, q4_y_coord,

\$ q4_z_coord, irot6, 'ONLY')

call gspos ('Q4FE', 3, 'HALL', -q4_x_coord, q4_y_coord,

\$ -q4_z_coord, irot7, 'ONLY')

call gspos ('Q4FE', 4, 'HALL', g4_x_coord, q4_y_coord,

$\$ \quad-\mathrm{q} 4{ }^{4} z_{-}$coord, irot8, 'ONLY')

C

$\mathrm{C}$

$\mathrm{C}$

$\mathrm{C}$

$\mathrm{C}$

$\mathrm{C}$

$\mathrm{C}$

C

C

C

C

\section{Install Q5. First, calculate the coordinates of the geometrical center of the magnet.}

q5_x_coord $=q 4$ 4 $x$ coord $-q 4$ co5_dist * sind (qsa_theta_deg) q5_z_coord $=$ q4_z_coord + q4_q5_dist $*$ cosd (qsa_theta_deg) qsa_par(1) = qsa_iron_ir

qsa_par (2) = qsa_iron_or

qsa_par $(3)=$ q5_length

nmed $=$ nmed_dx

call gsvolu ('Q5FE', 'TUBE', nmed, gsa_par, 3, ivolu)

call gsatt ('Q5FE', 'COLO', 6)

call gspos ('Q5FE', 1, 'HALL', q5_x_coord, q5_y_coord, $\$$ q5_z_coord, irot5, 'ONLY')

Install the collar inside 25 .

qsa_par $(1)=$ qsa_collar_ir

qsa_par $(2)$ = qsa_collar_or

nme $\bar{d}=$ nmed_gI0

call gsvolu ('Q5CL', 'TUBE', nmed, qsa_par, 3, ivolu)

call gsatt ('Q5CL', 'COLO', 6)

call gsatt ('Q5CL', 'SEEN', 0)

call gspos ('Q5CL', 1, 'Q5FE', $0.0 e 0,0.0 e 0,0.0 e 0,0$, 'ONLY')

Now install the coil inside Q5.

qsa_par(1) = qsa_coil_ir

qsa_par (2) = qsa_coil_or

nmed = nmed_cu

call gsvolu ('Q5CO', 'TUBE', nmed, qsa_par, 3, ivolu)

call gsatt ('Q5CO', 'COLO', 6)

call gsatt ('Q5CO', 'SEEN', 0)

call gspos ('Q5CO', 1, 'Q5CL', $0.0 e 0,0.0 e 0,0.0 e 0,0$, 'ONLY')

Install the gap between the beam tube and the coil.

qsa_par (1) = qsa_gap_ir

qsa_par (2) = qsa_gap_or

nmed $=16$

call gsvolu ('Q5GA', 'TUBE', nmed, qsa_par, 3, ivolu)

call gsatt ('Q5GA', 'COLO', 6)

call gsatt ('Q5GA', 'SEEN', 0)

call gspos ('Q5GA', 1, 'Q5CO', $0.0 e 0,0.0 e 0,0.0 e 0,0$, 'ONLY')

Now install the beam tube inside Q5.

qsa_par(1) = qsa_tube_ir

qsa_par $(2)$ q qsa_tube_or

nmed $=900$

call gsvolu ('Q5TU', 'TUBE', nmed, qsa_par, 3, ivolu)

call gsatt ('Q5TU',' 'COLO', 6)

call gsatt ('Q5TU', 'SEEN', O)

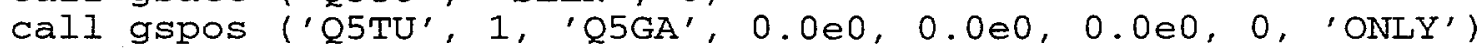

C

$\mathrm{C}$

Install the vacuum inside the tube. 
qsa_par $(1)=0.0 e 0$

qsa_par (2) = qsa_tube_ir

nmed $=16$

call gsvolu ('Q5VA', 'TUBE', nmed, qsa_par, 3, ivolu)

call gsatt ('Q5TU',' 'SEEN', 0$)$

call gspos ('Q5VA', 1, 'Q5TU', $0.0 e 0,0.0 e 0,0.0 e 0,0$, 'ONLY')

Install the other Q5 magnets.

call gspos ('Q5FE', 2, 'HALL', -q5_x_coord, q5_y_coord,

call gspos ('Q5FE', 3, 'HALL', -q5_x_coord, q5_y_coord,

$\$ \quad-q 5$ z_coord, irot7, 'ONLY')

call gspos ('Q5FE', 4, 'HALL', q5_x_coord, q5_y_coord,

$\mathrm{C}$

$\mathrm{C}$

C

$\mathrm{C}$

Install D5. First, calculate the coordinates of the geometrical centers of the two magnets. First the outer arc.

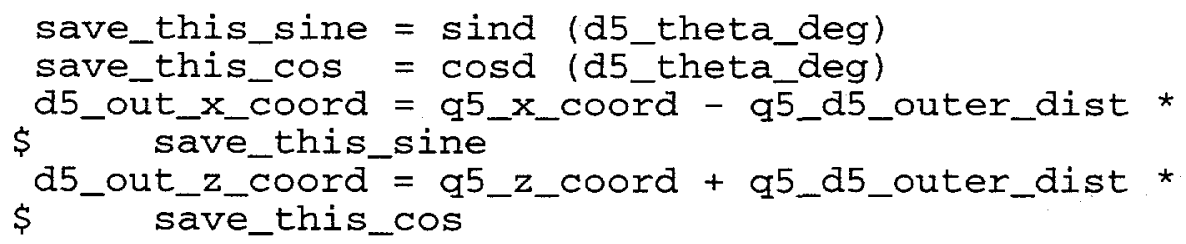

Now calculate the coordinates of the magnet in the inner arc.

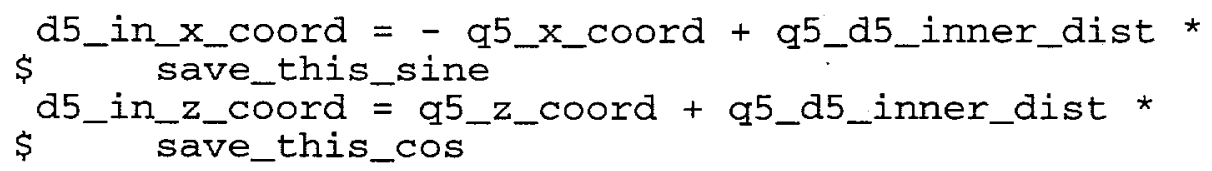

Now install the magnet in the outer arc.

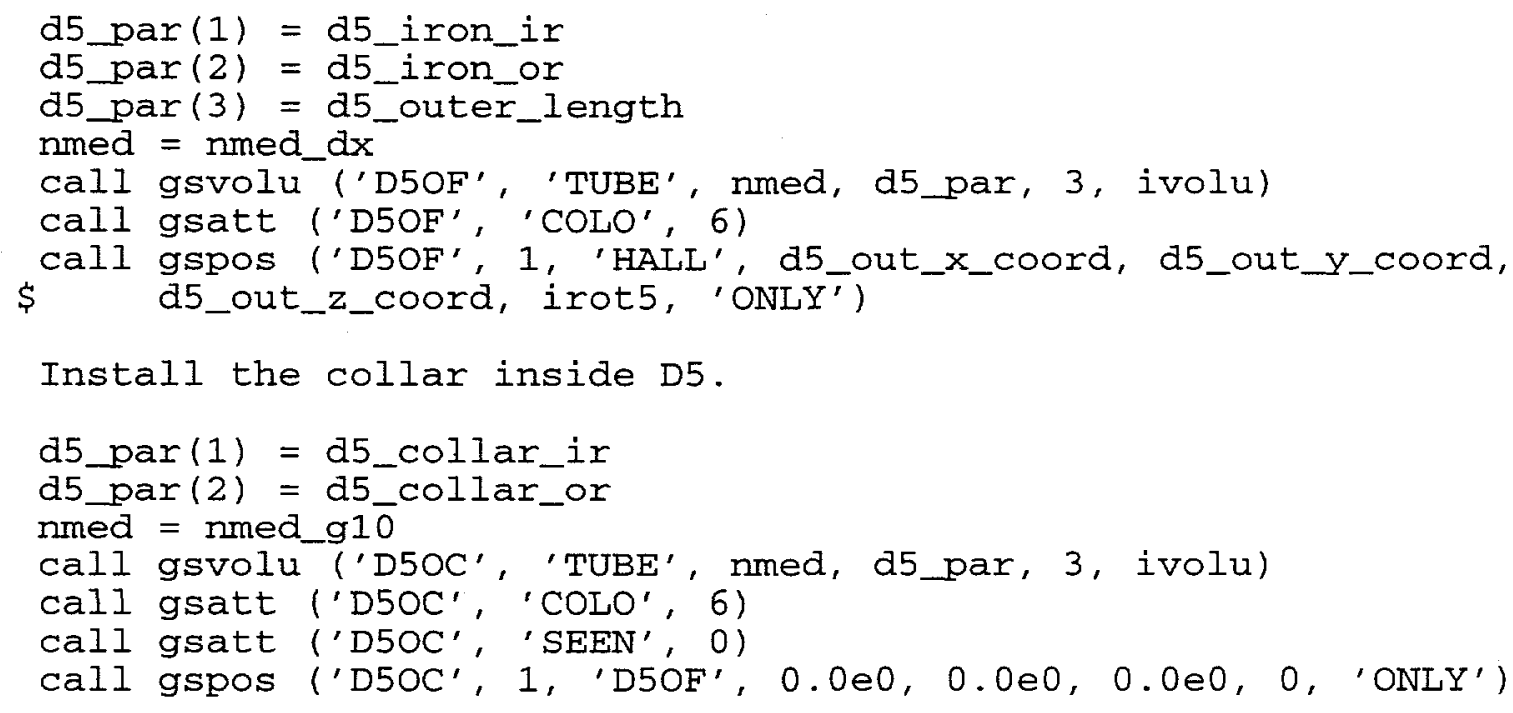


d5_par (1) = d5_gap_ir

d5_par (2) = d5_gap_or

nmed $=16$

call gsvolu ('D50G', 'TUBE', nmed, d5_par, 3, ivolu)

call gsatt ('D5OG', 'COLO', 6)

call gsatt ('D5OG', 'SEEN', 0)

call gspos ('D5OG', 1, 'D5OL', 0.0e0, $0.0 e 0,0.0 e 0,0$, 'ONLY')

C

C

Now install the beam tube inside the outer D5.

d5_par (1) = a5_tube_ir

$d 5$ _par $(2)=d 5$ _tube_or

nmed $=900$

call gsvolu ('D50T', 'TUBE', nmed, d5_par, 3, ivolu)

call gsatt ('D5OT', 'COLO', 6)

call gsatt ('D5OT', 'SEEN', 0)

call gspos ('D5OT', 1, 'D5OG', $0.0 e 0,0.0 e 0,0.0 e 0,0$, 'ONLY')

Install the vacuum inside the tube.

d5_par $(1)=0.0 e 0$

d5_par (2) = d5_tube_ir

nmed $=16$

call gsvolu ('D50V', 'TUBE', nmed, d5_par, 3, ivolu)

call gsatt ('D50V', 'SEEN', 0)

call gspos ('D5OV', 1, 'D5OT', $0.0 \mathrm{e} 0,0.0 \mathrm{e} 0,0.0 \mathrm{e} 0,0$, 'ONLY')

Install the inner D5 magnet.

d5_par $(1)=$ d5_iron_ir

d5_par (2) = d5_iron_or

$\mathrm{d} 5$ _par $(3)=\mathrm{d} 5$ _inner_length

nmed = nmed_dx

call gsvolu ('D5IF', 'TUBE', nmed, d5_par, 3, ivolu)

call gsatt ('D5IF', 'COLO', 6)

call gspos ('D5IF', 1, 'HALL', d5
$\$$

call gspos ('D5IF', 1, 'HALL', d5_in_x_coord, d5_in_y_coord,

Install the collar inside the inner D5.

d5_par $(1)=d 5$ collar_ir

d5_par $(2)=$ d5_collar_or

nmed = nmed_g10

call gsvolu ('D5IC', 'TUBE', nmed, d5_par, 3, ivolu)

call gsatt ('D5IC', 'COLO', 6)

call gsatt ('D5IC', 'SEEN', 0 )

call gspos ('D5IC', 1, 'D5IF', 0.0e0, $0.0 e 0,0.0 e 0,0, '$ ONLY')

$\mathrm{C}$

$\mathrm{C}$

Now install the coil inside inner D5.

d5_par $(1)=$ d5_coil_ir

d5_par $(2)=$ d5_coil_or

nmed = nmed_cu

call gsvolu ('D5IL', 'TUBE', nmed, d5_par, 3, ivolu)

call gsatt ('D5II', 'COLO', 6)

call gsatt ('D5IL', 'SEEN', 0 )

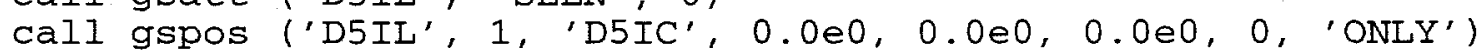

Install the gap between the beam tube and the coil in the inner D5 magnet.

d5_par $(1)=$ d5_gap_ir

d5_par $(2)=$ d5_gap_or

nmed $=16$

call gsvolu ('D5IG', 'TUBE', nmed, d5_par, 3, ivolu) 
call gsatt ('D5IG', 'COLO', 6)

call gsatt ('D5IG', 'SEEN', 0)

call gspos ('D5IG', 1, 'D5IL', 0.0e0, $0.0 e 0,0.0 e 0,0$, 'ONLY')

C

$\mathrm{C}$

$\mathrm{C}$

$\mathrm{C}$

C

C

C

$\mathrm{C}$

C

C

C

C

C

C

C

C

C

C

C

C

C

C

C

C

C

Now install the beam tube inside the inner D5.

d5_par $(1)=$ d5_tube_ir

d5_par (2) = d5_tube_or

nmed $=900$

call gsvolu ('D5IT', 'TUBE', nmed, d5_par, 3, ivolu)

call gsatt ('D5IT', 'COLO', 6)

call gsatt ('D5IT', 'SEEN', 0)

call gspos ('D5IT', 1, 'D5IG', $0.000,0.000,0.0 e 0,0$, 'ONLY')

Install the vacuum inside the tube.

d5_par $(1)=0.0 \mathrm{e} 0$

d5_par (2) = d5_tube_ir

nmed $=16$

call gsvolu ('D5IV', 'TUBE', nmed, d5_par, 3, ivolu)

call gsatt ('D5IV', 'SEEN', 0)

call gspos ('D5IV', 1, 'D5IT', 0.0e0, 0.0e0, 0.0e0, 0, 'ONLY')

Install the corresponding magnets on the other side of the origin.

call gspos ('D5IF', 2, 'HALL', d5_in_x_coord, d5_in_y_coord,

$\$$-d5_in_z_coord, irot5, 'ONLY' ')

call gspos ('D5OF',2, 'HALL', d5_out_x_coord, d5_out_y_coord, $\$$ -d5_out_z_coord, irot6, 'ONLY')

For purposes of alignment we will now change the values of the variables d5_in_x_coord, d5_in_z_coord, d5_out_x_coord, and d5_out_z_coord. We want to point the following magnets towards the downstream end of D5, not towards its center. At the same time we do this, though, we must subtract the half lengths of the magnets from d5_q6_in_dist, etc., in order to keep the distances between magnets constant.

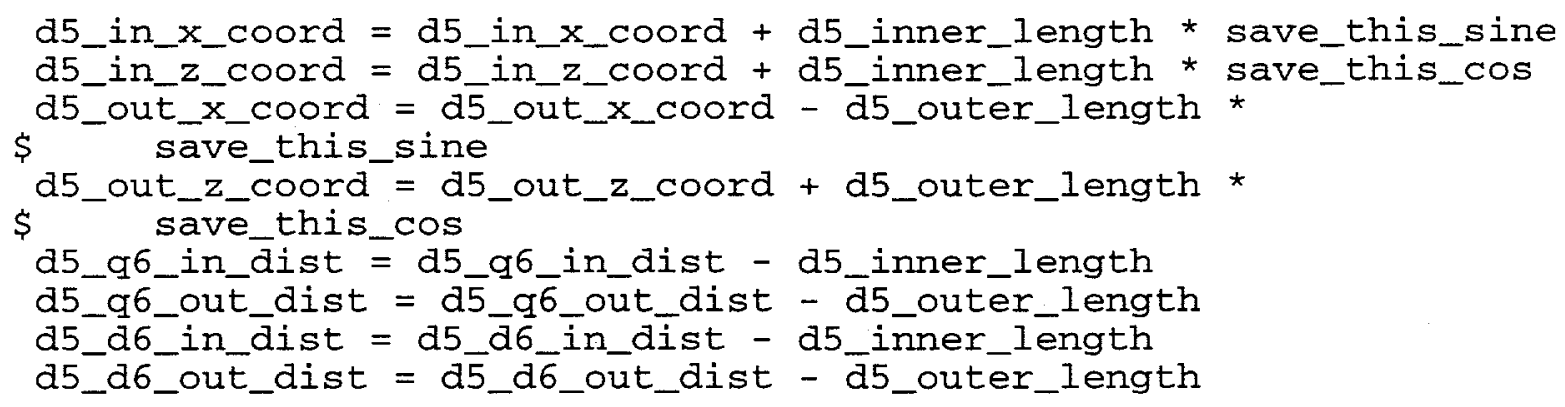

Install 06 in the inner and outer arcs. First, calculate the coordinates of the centers.

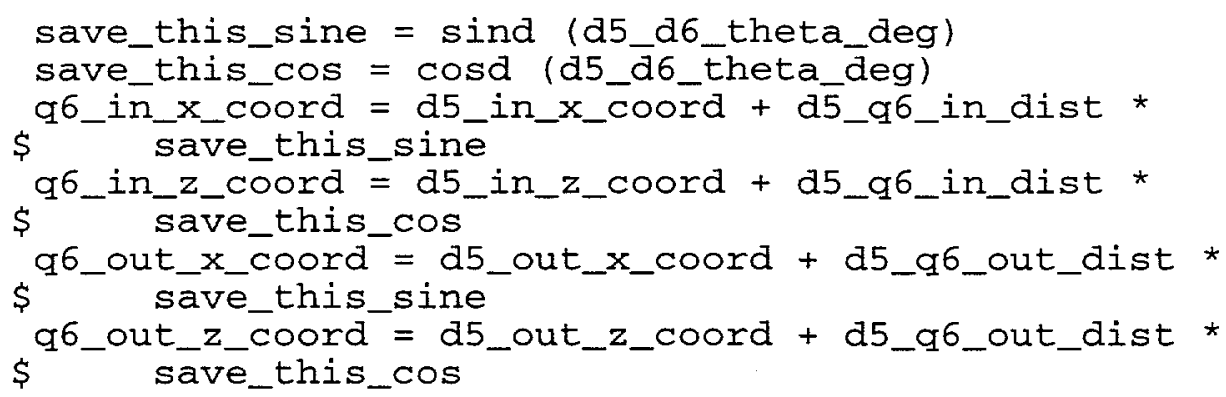

Now install $\mathrm{Q} 6$ in the inner arc. Note that we need a new rotation matrix. 
call gsatt ('D5IG', 'COLO', 6)

call gsatt ('D5IG', 'SEEN', 0)

call gspos ('D5IG', 1, 'D5IL', $0.0 e 0,0.0 e 0,0.0 e 0,0$, 'ONLY')

C

C

C

$\mathrm{C}$

$\mathrm{C}$

C

C

C

C

C

C

C

C

C

C

C

$\mathrm{C}$

C

Now install the beam tube inside the inner D5.

d5_par (1) $=$ d5_tube_ir

d5_par $(2)=$ d5_tube_or

nmed $=900$

call gsvolu ('D5IT', 'TUBE', nmed, d5 par, 3, ivolu)

call gsatt ('D5IT', 'COLO', 6)

call gsatt ('D5IT', 'SEEN', 0 )

call gspos ('D5IT', 1, 'D5IG', 0.0e0, 0.0e0, 0.0e0, 0, 'ONLY')

Install the vacuum inside the tube.

d5_par(1) $=0.000$

d5_par $(2)=$ d5_tube_ir

nmed $=16$

call gsvolu ('D5IV', 'TUBE', nmed, d5_par, 3, ivolu)

call gsatt ('D5IV', 'SEEN', 0 )

call gspos ('D5IV', 1, 'D5IT', $0.0 e 0,0.0 e 0,0.0 e 0,0$, 'ONLY') $^{2}$

Install the corresponding magnets on the other side of the origin.

call gspos ('D5IF', 2, 'HALL', d5_in_x_coord, d5_in_y_coord,

$\$$

call gspos ('D5OF', 2, 'HALL', d5_out_x_coord, d5_out_y_coord,

$\$$-d5_out_z_coord, irot6,' 'ONLY')

For purposes of alignment we will now change the values of the variables d5_in_x_coord, d5_in_z_coord, d5_out_x_coord, and d5_out_z_coord. We want to point the following magnets towards the downstream end of D5, not towards its center. At the same time we do this, though, we must subtract the half lengths of the magnets from d5_q6_in_dist, etc., in order to keep the distances between magnets constant.

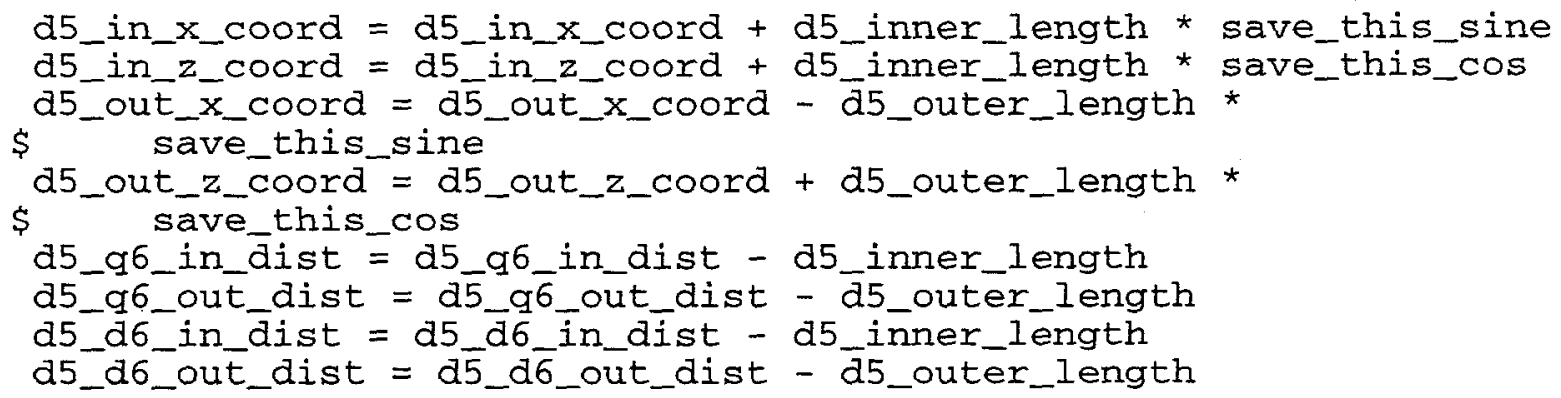

Install Q6 in the inner and outer arcs. First, calculate the coordinates of the centers.

save_this_sine $=$ sind (d5_d6_theta_deg)

save_this_cos $=\operatorname{cosd}$ (d5_d 6 _theta_deg)

q6_in_x_coord $=$ d5_in_x_coord $+d \overline{5} \_q 6$ _in_dist *

$\$$ save_this_sine

q6_in_z_coord $=$ d5_in_z_coord + d5_q6_in_dist *

$\$$ save_this_cos

q6_out_x_coord $=d^{6}$ _out_x_coord + d5_q6_out_dist *

$\$$ save_this_sine

q6_out_z_coord = d5_out_z_coord + d5_q6_out_dist *

$\$$ save_this_cos

Now install $\mathrm{Q} 6$ in the inner arc. Note that we need a new rotation matrix. 
C

C

$\mathrm{C}$

qsa_par(1) = qsa_iron_ir

qsa_par (2) = qsa_iron_or

qsa_par (3) = q6_length

nmed = nmed_dx

call gsvolu ('Q60F', 'TUBE', nmed, qsa_par, 3, ivolu)

call gsatt ('Q60F', 'COLO', 6)

call gspos ('Q60F', 1, 'HALL', q6_out_x_coord, q6_out_y_coord, $\$$ q6_out_z_coord, irot9, 'ONLY')

Install the collar inside the outer Q6.

qsa_par(1) = qsa_collar_ir

qsa_par $(2)$ = qsa_collar_or

nmed $=$ nmed_g10

call gsvolu ('Q60C', 'TUBE', nmed, qsa_par, 3, ivolu)

call gsatt ('Q60C', 'COLO', 6)

call gsatt ('Q60C', 'SEEN', 0 )

call gspos ('Q60C', 1, 'Q6OF', $0.0 e 0,0.0 e 0,0.0 e 0,0$, 'ONLY')

Now Onstall the coil inside the outer $Q 6$.

qsa_par(1) = qsa_coil_ir

qsa_par (2) = qsa_coil_or

nmed $=$ nmed_cu

call gsvolu ('Q60L', 'TUBE', nmed, qsa_par, 3, ivolu)

call gsatt ('Q60L', 'COLO', 6)

call gsatt ('Q60L', 'SEEN', 0$)$

call gspos ('Q60L', 1, 'Q60C', $0.0 e 0,0.0 e 0,0.0 e 0,0$, 'ONLY') $^{\prime}$

Install the gap between the beam tube and the coil.

qsa_par(1) = qsa_gap_ir

qsa_par $(2)$ = qsa_gap_or

nme $\bar{d}=16$

call gsvolu ('Q60G', 'TUBE', nmed, qsa_par, 3, ivolu)

call gsatt ('Q60G', 'COLO', 6)

call gsatt ('Q60G', 'SEEN', 0)

call gspos ('Q60G', 1, 'Q60L', $0.0 e 0,0.0 e 0,0.0 e 0,0$, 'ONLY')

Now install the beam tube inside the outer $Q 6$.

qsa_par(1) = qsa_tube_ir

qsa_par $(2)$ = qsa_tube_or

nmed $=900$

call gsvolu ('Q60T', 'TUBE', nmed, qsa_par, 3, ivolu)

call gsatt ('Q60T', 'COLO', 6)

call gsatt ('Q60T', 'SEEN', 0)

call gspos ('Q60T', 1, 'Q60G', 0.0e0, 0.0e0, $0.0 \mathrm{e} 0,0$, 'ONLY')

Install the vacuum inside the tube.

qsa_par $(1)=0.0 e 0$

qsa_par $(2)=$ qsa_tube_ir

nmed $=16$

call gsvolu ('Q60V', 'TUBE', nmed, qsa_par, 3, ivolu)

call gsatt ('Q60V', 'SEEN', 0)

call gspos ('Q60V', 1, 'Q60T', 0.0e0, 0.0e0, 0.0e0, 0, 'ONLY')

Install the magnets on the other side of the beam line.

We need a new rotation matrix.

angle1 $=90.0 e 0-$ d5_d6_theta_deg

angle2 = d5_d6_theta_deg

irot $=$ irot $+\overline{1}$

irot10 = irot

call gsrotm (irot10, angle1, 0.0e0, 90.0e0, 90.0e0, angle2, 
C

C

C

C

C

C

C

C

C

C

C

C

C

C

C

C

C

C

$\$ \quad 180.0 \mathrm{e} 0)$

call gspos ('Q6IF',2, 'HALL', q6_in_x_coord, q6_in_y_coord,

$\$ \quad-q 6$ in_z_coord, irot10, 'ONIY')

cal1 gspos ('Q60F',2, 'HALL', q6_out_x_coord, q6_out_y_coord,

$\$ \quad-q 6 \_o u t \_z \_c o o r d, \quad i \operatorname{rot} 10$, 'ONLY')

Begin installation of D6. first, calculate the coordinates of the centers of the magnets.

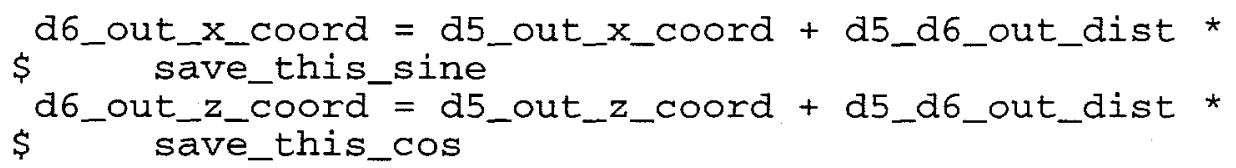

Now calculate the coordinates of the magnet in the inner arc.

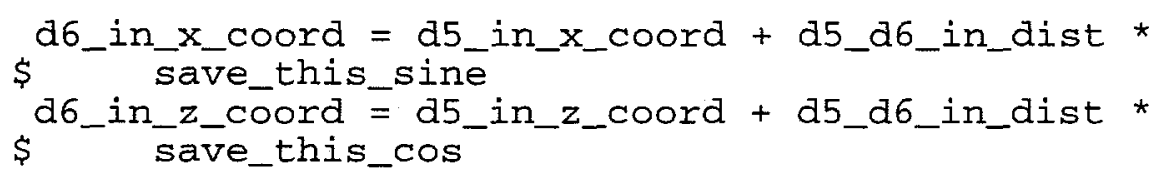

Now install the magnet in the outer arc.

d6_par(1) = d5_iron_ir

d6_par (2) = d5_iron_or

d6_par (3) = d6_length

nmed = nmed_dx

call gsvolu ('D6OF', 'TUBE', nmed, d6_par, 3, ivolu)

call gsatt ('D6OF', 'COLO', 6)

call gspos ('D60F', 1, 'HALL', d6_out_x_coord, d6_out_y_coord, $\$$ d6_out_z_coord, irot9, 'ONLY')

Install the collar inside the outer D6.

d6_par $(1)=$ d5_collar_ir

d6_par $(2)=$ d5_collar_or

nmed $=$ nmed_g $1 \overline{0}$

call gsvolu ('D60C', 'TUBE', nmed, d6_par, 3, ivolu)

call gsatt ('D60C', 'COLO', 6)

call gsatt ('D60C', 'SEEN', 0 )

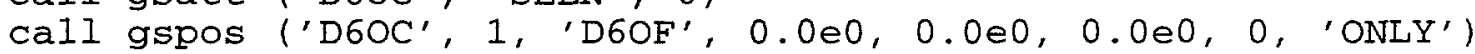

Now install the coil inside outer D6.

d6_par $(1)=$ d5_coil_ir

d6_par $(2)=d 5$ coil_or

nmed = nmed_cu

call gsvolu ('D60L', 'TUBE', nmed, d6_par, 3, ivolu)

cal1 gsatt ('D6OL', 'COLO', 6)

call gsatt ('D6OL', 'SEEN', 0)

call gspos ('D60L', 1, 'D6OC', 0.0e0, $0.0 e 0,0.0 e 0,0$, 'ONLY')

Install the gap between the beam tube and the coil in the outer magnet.

d6_par $(1)=$ d5_gap_ir

d6_par $(2)=$ d5_gap_or

nimed $=16$

call gsvolu ('D60G', 'TUBE', nmed, d6_par, 3, ivolu)

call gsatt ('D6OG', 'COLO', 6)

call gsatt ('D6OG', 'SEEN', 0)

call gspos ('D60G', 1, 'D6OL', 0.0e0, $0.000,0.0 e 0,0$, 'ONLY')

Now install the beam tube inside the outer $D 6$.

d6_par $(1)=$ d5_tube_ir 
d6_par $(2)=$ d5_tube_or

nmed $=900$

call gsvolu ('D60T', 'TUBE', nmed, d6_par, 3, ivolu)

call gsatt ('D6OT', 'COLO', 6)

call gsatt ('D6OT', 'SEEN', 0)

call gspos ('D60T', 1, 'D60G', 0.0e0, $0.0 e 0,0.0 e 0,0$, 'ONLY')

$\mathrm{C}$

C

$\mathrm{C}$

C

C

$\mathrm{C}$
$\mathrm{C}$
$\mathrm{C}$

$\mathrm{C}$

C

C

$\mathrm{C}$

C

$\mathrm{C}$

C

$\mathrm{C}$

C

Install the vacuum inside the tube.

d6_par $(1)=0.0 e 0$

d6_par $(2)=d 5$ _tube_ir

nmed $=16$

call gsvolu ('D60V', 'TUBE', nmed, d6_par, 3, ivolu)

call gsatt ('D6OV', 'SEEN', O)

call gspos ('D6OV', 1, 'D6OT', $0.0 \mathrm{e}, 0.0 \mathrm{e}, 0.0 \mathrm{e}, 0, \mathrm{O}^{\mathrm{O}} \mathrm{ONLY}$ ')

Instal1 the inner D6 magnets.

d6_par $(1)=d 5 \_i r o n \_i r$

d6_par $(2)=d 5$ iron_or

d6_par $(3)=d 6$ length

nmed = nmed_dx

call gsvolu ('D6IF', 'TUBE', nmed, d6_par, 3, ivolu)

call gsatt ('D6IF', 'COLO', 6)

call gspos ('D6IF', 1, 'HALL', d6_in_x_coord, d6_in_Y_coord, $\$$ d6_in_z_coord, irot9, 'ONLY')

Install the collar inside the inner D6.

d6_par $(1)=$ d5_collar_ir

d6_par $(2)=d 5$ collar_or

nmed = nmed_g10

call gsvolu ('D6IC', 'TUBE', nmed, d6_par, 3, ivolu)

call gsatt ('D6IC', 'COLO', 6)

call gsatt ('D6IC', 'SEEN', 0)

call gspos ('D6IC', 1, 'D6IF', 0.0e0, 0.0e0, $0.0 \mathrm{e} 0,0$, 'ONLY')

Now install the coil inside inner D6.

d6_par $(1)=$ d5_coil_ir

d6_par $(2)=$ d5_coil_or

nmed = nmed_cu

call gsvolu ('D6IL', 'TUBE', nmed, d6_par, 3, ivolu)

call gsatt ('D6IL', 'COLO', 6)

call gsatt ('D6IL', 'SEEN', 0)

call gspos ('D6IL', 1, 'D6IC', 0.0e0, 0.0e0, $0.0 e 0,0$, 'ONLY')

Install the gap between the beam tube and the coil in the inner D6 magnet.

d6_par (1) = d5_gap_ir

d6_par $(2)=$ d5_gap_or

nmed $=16$

call gsvolu ('D6IG', 'TUBE', nmed, d6_par, 3, ivolu)

call gsatt ('D6IG', 'COLO', 6)

call gsatt ('D6IG', 'SEEN', 0)

call gspos ('D6IG', 1, 'D6IL', $0.0 e 0,0.0 e 0,0.0 e 0,0$, 'ONLY')

Now install the beam tube inside the inner D6.

d6_par (1) = d5_tube_ir

d6_par (2) = d5_tube_or

nmed $=900$

call gsvolu ('D6IT', 'TUBE', nmed, d6_par, 3, ivolu)

call gsatt ('D6IT', 'COLO', 6)

call gsatt ('D6IT', 'SEEN', 0 ) 
call gspos ('D6IT', 1, 'D6IG', $0.0 e 0,0.0 e 0,0.0 e 0,0$, 'ONLY')

Install the vacuum inside the tube.

d6 par $(1)=0.0 \mathrm{e} 0$

d6_par $(2)=$ d5_tube_ir

nmed $=16$

call gsvolu ('D6IV', 'TUBE', nmed, d6_par, 3, ivolu)

call gsatt ('D6IV', 'SEEN', 0)

call gspos ('D6IV', 1, 'D6IT', 0.0e0, $0.0 e 0,0.0 e 0,0$, 'ONLY')

C

$\mathrm{C}$

C

C

C

C

C

C

C

C

C

$\mathrm{C}$

C

C

$\mathrm{C}$
$\mathrm{C}$
$\mathrm{C}$
$\mathrm{C}$

Install the corresponding magnets on the other side of the origin.

call gspos ('D6IF',2, 'HALL', d6_in_x_coord, d6_in_y_coord, $\$$ -d6_in_z_coord, irot10, ' ONLY')

call gspos ('D60F',2, 'HALL', d6_out_x_coord, d6_out_y_coord, \$ -d6_out_z_coord, irot10, 'ONLY')

End installation of D6. Begin installation of $Q 7$

For purposes of alignment we will now change the values of the variables d6_in_x_coord, d6_in_z_coord, d6_out_x_coord, and d6_out_z_coord. We want to point the following magnets towards the downstream end of D6, not towards its center. At the same time we do this, though, we must subtract the half lengths of the magnets from d6_q7_in_dist, etc., in order to keep the distances between magnets constant.

d6_in_x_coord $=d 6$ _in_x_coord $+d 6 \_$length * save_this_sine d6_in_z_coord $=d 6$ _in_z_coord $+d 6$ _length $*$ save_this_cos

$d 6$ _out_x_coord $=\mathrm{d} \overline{6}$ _out_x_coord $+\bar{d} 6$ _length $*$ save_this_sine

d6_out_z_coord $=d 6$ _out_z_coord + d6_length $*$ save_this_cos

d6_q7_in_dist $=$ d6_q7 in_dist $-d 6$ length

d6_q7_out_dist $=\mathrm{d} \bar{\sigma}-q \overline{7}$ _out_dist $-\overline{\mathrm{d}} \sigma_{\text {_length }}$

d6_q8_in_dist $=\mathrm{d} 6$-q8_in_dist $-\mathrm{d} 6$ _length

d6_q8_out_dist $=\mathrm{d} \overline{6}$ _q $\overline{8}$ _out_dist $-\overline{\mathrm{d}} \sigma_{\text {_l }}$ length

d6_d8_in_dist $=$ d6_d8_in_dist $-d 6$ length

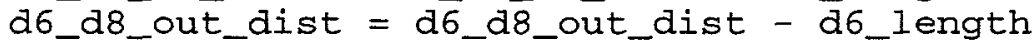

Now install $Q 7$ in the inner arc. First define the new rotation matrices.

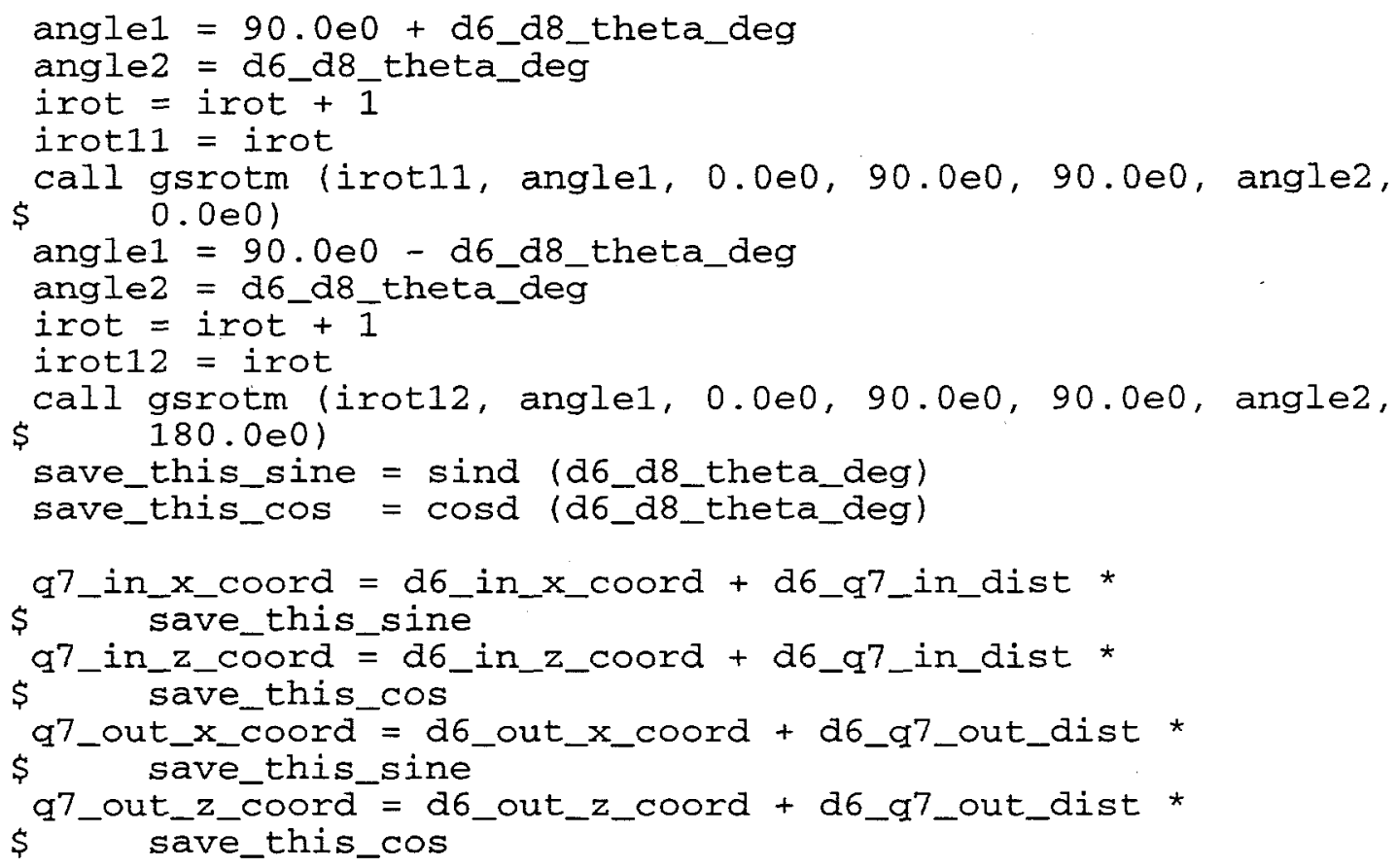


qsa_par(1) = qsa_iron_ir

qsa_par (2) = qsa_iron_or

qsa_par $(3)=$ q7_length

nme $\bar{d}=$ nmed_dx

call gsvolu ('Q7IF', 'TUBE', nmed, qsa_par, 3, ivolu)

call gsatt ('Q7IF', 'COLO', 6)

$\$$

call gspos ('Q7IF', 1, 'HALL', q7_in_x_coord, q7_in_y_coord,

$\mathrm{C}$

C

Install the collar inside the inner Q7.

qsa_par(1) = qsa_collar_ir

qsa_par $(2)$ = qsa_collar_or

nmed $=$ nmed_g10

call gsvolu ('Q7IC', 'TUBE', nmed, qsa_par, 3, ivolu)

call gsatt ('Q7IC', 'COLO', 6)

call gsatt ('Q7IC', 'SEEN', 0 )

call gspos ('Q7IC', 1, 'Q7IF', $0.0 e 0,0.0 e 0,0.0 e 0,0$, 'ONLY')

$\mathrm{C}$

C

$\mathrm{C}$

$\mathrm{C}$

C

$\mathrm{C}$

$\mathrm{C}$

$\mathrm{C}$

$\mathrm{C}$

$\mathrm{C}$

C

$\mathrm{C}$

C

Now install the coil inside the inner $Q 7$.

qsa_par $(1)=$ qsa_coil_ir

qsa_par $(2)$ = qsa_coil_or

nmed = nmed_cu

call gsvolu ('Q7IL', 'TUBE', nmed, qsa_par, 3, ivolu)

call gsatt ('Q7IL', 'COLO', 6)

call gsatt ('Q7IL', 'SEEN', 0 )

call gspos ('Q7IL', 1, 'Q7IC', 0.0e0, $0.0 e 0,0.0 e 0,0$, 'ONLY')

Install the gap between the beam tube and the coil.

qsa_par (1) = qsa_gap_ir

qsa_par $(2)$ = qsa_gap_or

nmed $=16$

call gsvolu ('Q7IG', 'TUBE', nmed, qsa_par, 3, ivolu)

call gsatt ('Q7IG', 'COLO', 6)

call gsatt ('Q7IG', 'SEEN', 0 )

call gspos ('Q7IG', 1, 'Q7IL', $0.0 e 0,0.0 e 0,0.0 e 0,0$, 'ONLY')

Now install the beam tube inside the inner $Q 7$.

qsa_par (1) = qsa_tube_ir

qsa_par $(2)=$ qsa_tube_or

nmed $=900$

call gsvolu ('Q7IT', 'TUBE', nmed, qsa_par, 3, ivolu)

call gsatt ('Q7IT', 'COLO', 6)

call gsatt ('Q7IT', 'SEEN', 0)

call gspos ('Q7IT', 1, 'Q7IG', 0.0e0, 0.0e0, 0.0e0, 0, 'ONLY')

Install the vacuum inside the tube.

qsa_par $(1)=0.0 e 0$

qsa_par $(2)=$ qsa_tube_ir

nmed $=16$

call gsvolu ('Q7IV', 'TUBE', nmed, qsa_par, 3, ivolu)

call gsatt ('Q7IV', 'SEEN', 0 )

call gspos ('Q7IV', 1, 'Q7IT', $0.0 e 0,0.0 e 0,0.0 e 0,0$, 'ONLY')

Now install the outer $Q 7$.

qsa_par(1) = qsa_iron_ir

qsa_par (2) = qsa_iron_or

qsa_par (3) = q7_Iength

nmed = nmed_dx

call gsvolu ('Q7OF', 'TUBE', nmed, qsa_par, 3, ivolu) 
C

$\mathrm{C}$

C

C C C

C C C

C

C

C

$C$
$C$

C

C

C

C

C

C

C

call gsatt ('Q7OF', 'COLO', 6) $\$$

call gspos ('Q7OF', 1, 'HALI', q7_out_x_coord, q7_out_y_coord, q7_out_z_coord, irot11, 'ONLY')

Install the collar inside the outer $Q 7$.

qsa_par $(1)=$ qsa_collar_ir

qsa_par (2) = qsa_collar_or

nmed $=$ nmed_g10

call gsvolu ('Q70C', 'TUBE', nmed, qsa_par, 3, ivolu)

call gsatt ('Q7OC', 'COLO', 6)

call gsatt ('Q7OC', 'SEEN', 0)

call gspos ('Q7OC', 1, 'Q7OF', $0.0 e 0,0.0 e 0,0.0 e 0,0, '$ ONLY')

Now Onstall the coil inside the outer Q7.

qsa_par $(1)=$ gsa_coil_ir

qsa_par $(2)=$ qsa_coil_or

nmed = nmed_cu

call gsvolu ('Q7OL', 'TUBE', nmed, qsa_par, 3, ivolu)

call gsatt ('Q7OL', 'COLO', 6)

call gsatt ('Q7OL', 'SEEN', 0)

call gspos ('Q7OL', 1, 'Q7OC', $0.0 \mathrm{e} 0,0.0 \mathrm{e} 0,0.0 \mathrm{e} 0,0$, 'ONLY')

Install the gap between the beam tube and the coil.

qsa_par $(1)=$ qsa_gap_ir

qsa_par $(2)=$ qsa_gap_or

nmed $=16$

call gsvolu ('Q7OG', 'TUBE', nmed, qsa_par, 3, ivolu)

call gsatt ('Q7OG', 'COLO', 6)

call gsatt ('Q7OG',' 'SEEN', 0)

call gspos ('Q7OG', 1, 'Q7ÓL', $0.0 e 0,0.0 e 0,0.0 e 0,0$, 'ONLY')

Now install the beam tube inside the outer Q7.

qsa_par(1) = qsa_tube_ir

qsa_par $(2)$ = qsa_tube_or

nmed $=900$

call gsvolu ('Q7OT', 'TUBE', nmed, qsa_par, 3, ivolu)

call gsatt ('Q7OT', 'COLO', 6)

call gsatt ('Q7OT', 'SEEN', 0 )

call gspos ('Q7OT', 1, 'Q7OG', $0.0 e 0,0.0 e 0,0.0 e 0,0$, 'ONLY')

Install the vacuum inside the tube.

qsa_par $(1)=0.0 \mathrm{e} 0$

qsa_par $(2)=$ qsa_tube_ir

nmed $=16$

call gsvolu ('Q70V', 'TUBE', nmed, qsa_par, 3, ivolu)

call gsatt ('Q7OV', 'SEEN', 0 )

call gspos ('Q7OV', 1, 'Q7OT', 0.0e0, 0.0e0, $0.0 \mathrm{e} 0,0$, 'ONLY')

Install the magnets on the other side of the origin.

call gspos ('Q7IF',2, 'HALL', q7_in_x_coord, q7_in_y_coord,

$\$$-q7_in_z_coord, irot12, 'ONLY')

call gspos ('O7OF',2, 'HALL', q7_out_x_coord, q7_out_y_coord, $\$$ -q7_out_z_coord, irot 12 , 'ONNLY')

End installation of $Q 7$.

Begin installation of $\mathrm{Q} 8$.

q8_in_x_coord $=d 6 \_i n \_x \_c o o r d+d 6 \_q 8$ in_dist * sind (d6_d8_theta_deg) 
C

C

$\mathrm{C}$

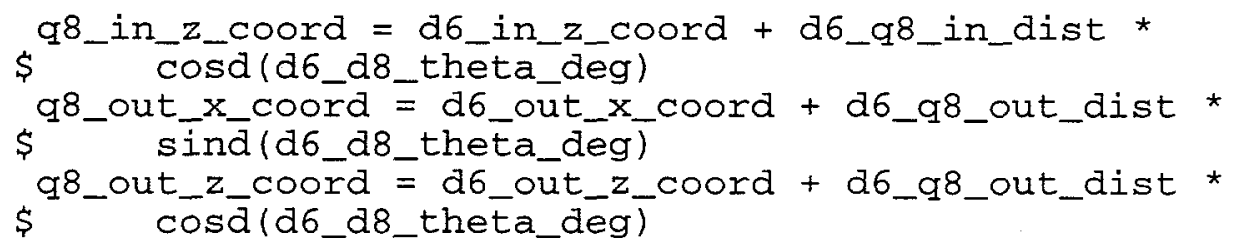

Install the collar inside the inner $Q 8$.

qsa_par $(1)=$ qsa_collar_ir

qsa_par $(2)$ = qsa_collar_or

nmed $=$ nmed_g10

call gsvolu ('Q8IC', 'TUBE', nmed, qsa_par, 3, ivolu)

call gsatt ('Q8IC', 'COLO', 6)

call gsatt ('Q8IC', 'SEEN', 0)

cail gspos ('Q8IC', 1, 'Q8IF', $0.000,0.0 e 0,0.000,0$, 'ONLY')

Now install the coil inside the inner $\mathrm{Q} 8$.

qsa_par(1) = qsa_coil_ir

qsa_par $(2)$ = qsa_coil_or

nmed $=$ nmed_cu

call gsvolu ('Q8IL', 'TUBE', nmed, qsa_par, 3, ivolu)

call gsatt ('Q8IL', 'COLO', 6)

call gsatt ('Q8IL', 'SEEN', 0 )

call gspos ('Q8IL', 1, 'Q8IC', $0.0 e 0,0.0 \mathrm{e} 0,0.0 \mathrm{e} 0,0$, 'ONLY')

Install the gap between the beam tube and the coil.

qsa_par $(1)=$ qsa_gap_ir

qsa_par (2) = qsa_gap_or

nmed $=16$

call gsvolu ('Q8IG', 'TUBE', nmed, qsa_par, 3, ivolu)

call gsatt ('Q8IG', 'COLO', 6)

call gsatt ('Q8IG', 'SEEN', 0 )

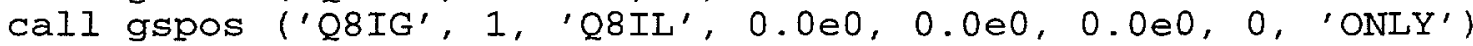

Now install the beam tube inside the inner $Q 8$.

qsa_par(1) = qsa_tube_ir

qsa_par $(2)$ = qsa_tube_or

nmed $=900$

call gsvolu ('Q8IT', 'TUBE', nmed, qsa_par, 3, ivolu)

call gsatt ('Q8IT', 'COLO', 6)

call gsatt ('Q8IT', 'SEEN', 0 )

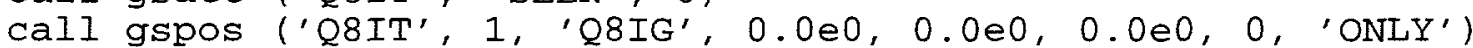

Install the vacuum inside the tube.

qsa_par(1) $=0.0 e 0$

qsa_par $(2)=$ qsa_tube_ir

nmed $=16$

call gsvolu ('Q8IV', 'TUBE', nmed, qsa_par, 3, ivolu)

call gsatt ('Q8IV',' 'SEEN', 0 ) 
call gspos ('Q8IV', 1, 'Q8IT', 0.0e0, 0.0e0, 0.0e0, 0, 'ONLY')

Now install the outer $\mathrm{Q} 8$.

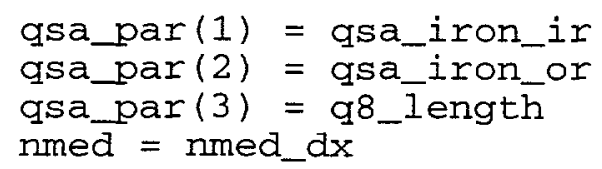

Install the collar inside the outer 08 .

qsa_par $(1)=$ qsa_collar_ir

qsa_par $(2)$ = qsa_collar_or

nmed = nmed_glo

call gsvolu ('Q80C', 'TUBE', nmed, gsa_par, 3, ivolu)

call gsatt ('Q80C', 'COLO', 6)

call gsatt ('Q80C', 'SEEN', 0 )

call gspos ('Q8OC', 1, 'Q8OF', $0.0 e 0,0.0 e 0,0.0 e 0,0$, 'ONLY')

$\mathrm{C}$

$\mathrm{C}$

$\mathrm{C}$

$\mathrm{C}$

C

C

$\mathrm{C}$

$\mathrm{C}$

C

C

C

C

$\mathrm{C}$

C

Now Onstall the coil inside the outer $Q 8$.

qsa_par(1) = qsa_coil_ir

qsa_par (2) = qsa_coil_or

nmed $=$ nmed_cu

call gsvolu ('Q80L', 'TUBE', nmed, qsa_par, 3, ivolu)

call gsatt ('Q8OL', 'COLO', 6)

call gsatt ('Q8OL', 'SEEN', 0 )

call gspos ('Q8OL', 1, 'Q8OC', $0.0 e 0,0.0 e 0,0.0 e 0,0$, 'ONLY')

Install the gap between the beam tube and the coil.

qsa_par(1) = qsa_gap_ir

qsa_par $(2)$ = qsa_gap_or

nmed $=16$

call gsvolu ('Q80G', 'TUBE', nmed, qsa_par, 3, ivolu)

call gsatt ('Q8OG', 'COLO', 6)

call gsatt ('Q8OG', 'SEEN', 0)

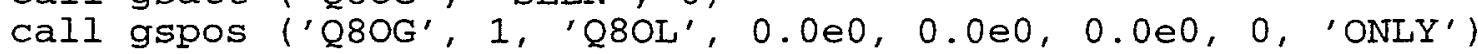

Now install the beam tube inside the outer Q8.

qsa_par (1) = qsa_tube_ir

qsa_par $(2)$ = qsa_tube_or

nmed $=900$

call gsvolu ('Q8OT', 'TUBE', nmed, qsa_par, 3, ivolu)

call gsatt ('Q8OT', 'COLO', 6)

call gsatt ('Q80T', 'SEEN', 0 )

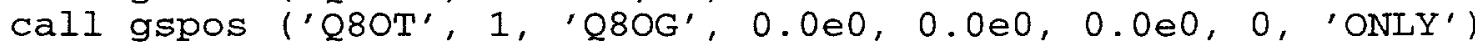

Install the vacuum inside the tube.

qsa_par (1) $=0.0 e 0$

qsa_par $(2)=$ qsa_tube_ir

nmed $=16$

call gsvolu ('Q8OV', 'TUBE', nmed, qsa_par, 3, ivolu)

call gsatt ('Q8OV', 'SEEN', 0 )

call gspos ('Q8OV', 1, 'Q8OT', 0.0e0, 0.0e0, 0.0e0, 0, 'ONLY')

Install the magnets on the other side of the origin.

call gspos ('Q8IF',2, 'HALL', q8_in_x_coord, q8_in_y_coord,

$\$$-q8_in_z_coord, irot 12,' ONLY') 
call gspos ('Q8OF', 2, 'HALL', q8_out_x_coord, q8_out_y_coord, -q8_out_z_coord, irot 12 , 'ONLY')

End installation of Q8.

Begin installation of D8.

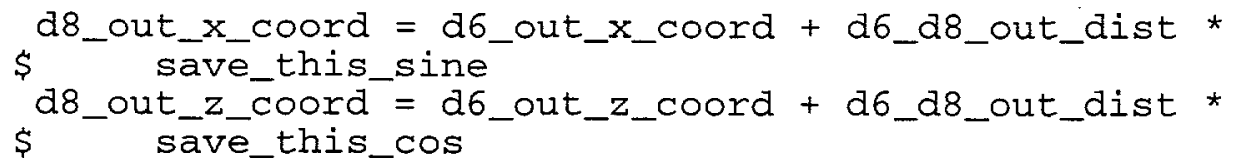

Now calculate the coordinates of the magnet in the inner arc. d8_in_x_coord $=$ d6_in_x_coord + d6_d8_in_dist * $\$$ save_this_sine d8_in_z_coord = d6_in_z_coord + d6_d8_in_dist * $\$$ save_this_cos

C

C

C

C

C

C

C

$\mathrm{C}$

C

C

C

Now install the magnet in the outer arc.

d8_par $(1)=$ a5_iron_ir

d8_par $(2)=$ d5_iron_or

d8_par $(3)=$ d8_length

nmed = nmed_dx

call gsvolu ('D80F', 'TUBE', nmed, d8_par, 3, ivolu)

call gsatt ('D8OF', 'COLO', 6)

call gspos ('D8OF', 1, 'HALL', d8_out_x_coord, d8_out_y_coord, $\$$ d8_out_z_coord, irot11, 'ONLY')

Install the collar inside the outer D8.

d8_par $(1)=$ d5_collar_ir

d8_par $(2)=$ d5_collar_or

nmed = nmed_g $1 \overline{0}$

call gsvolu ('D80C', 'TuBE', nmed, d8_par, 3, ivolu)

call gsatt ('D80C', 'COLO', 6)

call gsatt ('D8OC', 'SEEN', 0)

call gspos ('D80C', 1, 'D8OF', $0.0 e 0,0.0 e 0,0.0 e 0,0$, 'ONLY')

Now install the coil inside outer D8.

d8_par $(1)=$ d5_coil_ir

d8_par $(2)=$ d5_coil_or

nmed $=$ nmed_cu

call gsvolu ('D80L', 'TUBE', nmed, d8 par, 3, ivolu)

call gsatt ('D8OL', 'COLO', 6)

call gsatt ('D8OL', 'SEEN', 0)

call gspos ('D8OL', 1, 'D8OC', $0.000,0.0 e 0,0.0 e 0,0, '$ ONLY')

Install the gap between the beam tube and the coil in the outer magnet.

d8_par (1) = d5_gap_ir

a8_par $(2)=$ a5_gap_or

nmed $=16$

call gsvolu ('D8OG', 'TUBE', nmed, d8_par, 3, ivolu)

call gsatt ('D8OG', 'COLO', 6)

call gsatt ('D8OG', 'SEEN', 0)

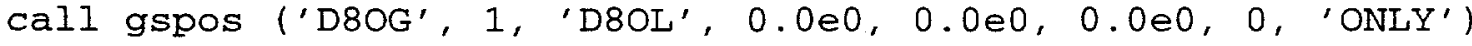

Now install the beam tube inside the outer D8.

d8_par (1) = d5_tube_ir

d8_par (2) = a5_tube_or 
nmed $=900$

call gsvolu ('D80T', 'TUBE', nmed, d8_par, 3, ivolu)

call gsatt ('D8OT', 'COLO', 6)

call gsatt ('D8OT', 'SEEN', 0)

call gspos ('D80T', 1, 'D8OG', $0.0 e 0,0.0 e 0,0.0 e 0,0$, 'ONLY')

C

$\mathrm{C}$

$\mathrm{C}$

C

C
C
C

C

C

$\mathrm{C}$

C

$\mathrm{C}$

C

$\mathrm{C}$

C

C

C

Install the vacuum inside the tube.

d8_par $(1)=0.0 e 0$

d8_par $(2)=$ d5_tube_ir

nmed $=16$

call gsvolu ('D8OV', 'TUBE', nmed, d8_par, 3, ivolu)

call gsatt ('D8OV', 'SEEN', 0)

call gspos ('D8OV', 1,'D8OT', $0.0 \mathrm{e} 0,0.0 \mathrm{e} 0,0.0 \mathrm{e} 0,0$, 'ONLY')

Install the inner D8 magnets.

d8_par (1) = d5 geriron_ir

d8_par (2) = d5_iron_or

d8_par $(3)=$ d8_length

nmed = nmed_dx

call gsvolu ('D8IF', 'TUBE', nmed, d8_par, 3, ivolu)

call gsatt ('D8IF', 'COLO', 6)

$\$$

call gspos ('D8IF', 1, 'HALL', d8_in_x_coord, d8_in_y_coord, d8_in_z_coord, irot11, 'ONLY')

Install the collar inside the inner D8.

d8_par $(1)=$ d5_collar_ir

d8_par $(2)=$ d5_collar_or

nmed $=$ nmed_glo

call gsvolu ('D8IC', 'TUBE', nmed, d8_par, 3, ivolu)

call gsatt ('D8IC', 'COLO', 6)

call gsatt ('D8IC', 'SEEN', 0)

call gspos ('D8IC', 1, 'D8IF', $0.000,0.0 e 0,0.000,0$, 'ONLY')

Now install the coil inside inner D8.

d8_par $(1)=$ d5_coil_ir

d8_par $(2)=$ d5_coil_or

nmed = nmed_cu

call gsvolu ('D8IL', 'TUBE', nmed, d8_par, 3, ivolu)

call gsatt ('D8IL', 'COLO', 6)

call gsatt ('D8IL', 'SEEN', 0 )

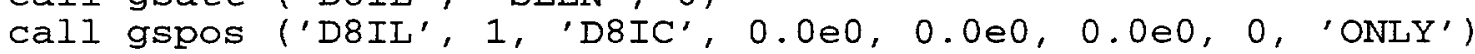

Install the gap between the beam tube and the coil in the inner D8 magnet.

d8_par $(1)=$ d5_gap_ir

d8_par $(2)=$ d5_gap_or

nmed $=16$

call gsvolu ('D8IG', 'TUBE', nmed, d8_par, 3, ivolu)

call gsatt ('D8IG', 'COLO', 6)

call gsatt ('D8IG', 'SEEN', 0 )

call gspos ('D8IG', 1 ,'D8IL', $0.0 \mathrm{e} 0,0.0 \mathrm{e} 0,0.0 \mathrm{e} 0,0$, 'ONLY')

Now install the beam tube inside the inner D8.

d8_par (1) = d5_tube_ir

d8_par $(2)=$ d5_tube_or

nmed $=900$

call gsvolu ('D8IT', 'TUBE', nmed, d8_par, 3, ivolu)

call gsatt ('D8IT', 'COLO', 6)

call gsatt ('D8IT', 'SEEN', 0)

call gspos ('D8IT', 1, 'D8IG', $0.0 e 0,0.0 e 0,0.0 e 0,0$, 'ONLY') 
Install the vacuum inside the tube.

d8_par $(1)=0.0 e 0$

d8_par $(2)=$ d5_tube_ir

nmed $=16$

call gsvolu ('D8IV', 'TUBE', nmed, d8_par, 3, ivolu)

call gsatt ('D8IV',' 'SEEN', 0 )

call gspos ('D8IV', 1, 'D8IT', 0.0e0, $0.0 e 0,0.0 e 0,0$, 'ONLY')

Install the corresponding magnets on the other side of the origin.

call gspos ('D8IF',2, 'HALL', d8_in_x_coord, d8_in_y_coord,

$\$$-d8_in_z_coord, irot 12,' 'ONLY')

call gspos ('D80F',2, 'HALL', d8_out_x_coord, d8_out_y_coord, $\$$-d8_out_z_coord, irot12, 'ONLY')

End installation of D8.

For purposes of alignment we will now change the values of the variables d8_in_x_coord, d8_in_z_coord, d8_out_x_coord, and d8_out_z_coord. We want to point the following magnets towards the downstream end of D8, not towards its center. At the same time we do this, though, we must subtract the half lengths of the magnets from d8_q9_in_dist, etc., in order to keep the distances between magnets constant.

d8_in_x_coord $=$ d8_in_x_coord + d8_length * save_this_sine d8_in_z_coord $=d 8$ _in_z_coord $+d 8$ _length $*$ save_this_cos d8_out_x_coord $=\mathrm{d} \overline{8}$ _out_x_coord $+\bar{d} 8$ _length $*$ save_this_sine d8_out_z_coord $=$ d8_out_z_coord + d8_length $*$ save_this_cos d8_q9_in_dist $=$ d8_q99_in_dist - d8_length

d8_q9_out_dist $=\mathrm{d} \overline{8} \_\mathrm{q} 9$ _out_dist $-\overline{\mathrm{d}} 8$ _length

d8_d9_in_dist $=$ d8_d9_in_dist - d8_length

d8_d9_out_dist $=$ d8_a $\overline{9} \_$out_dist $-\bar{d} 8 \_$length

Define required rotation matrices.

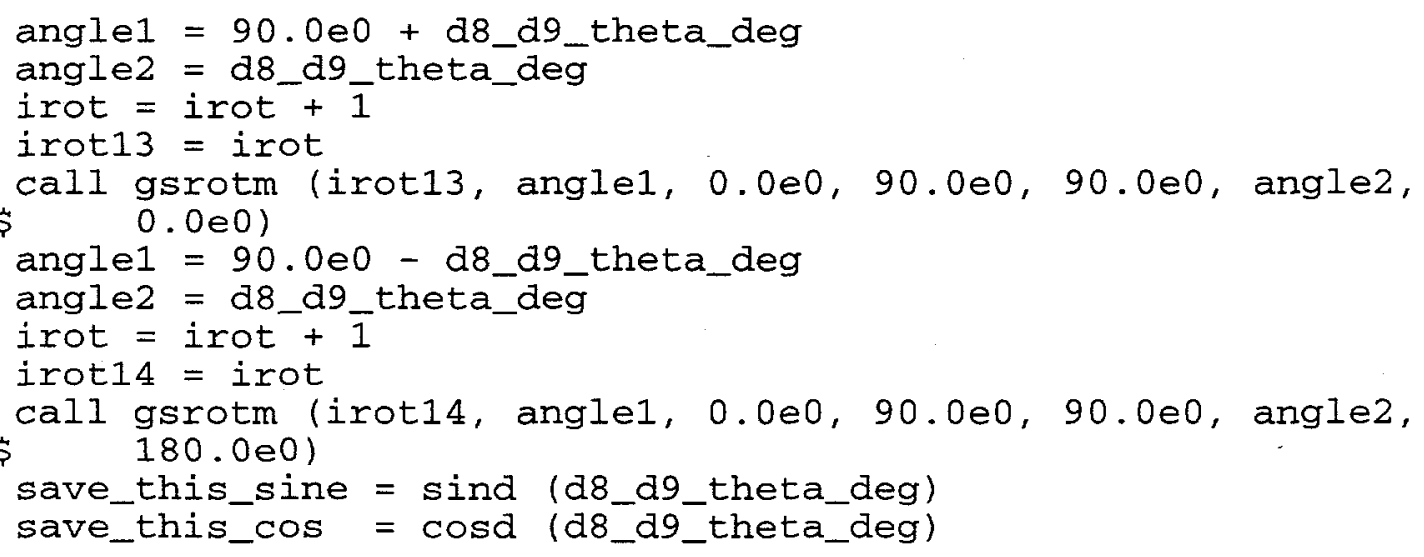

Begin installation of $\mathrm{Q} 9$.

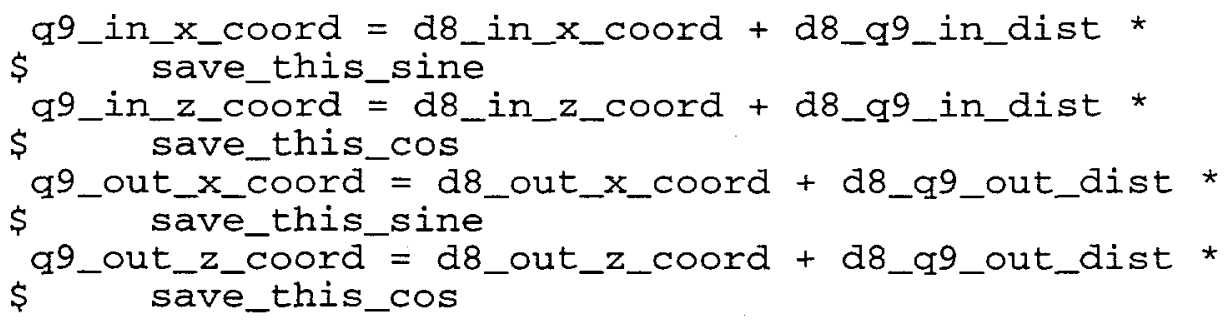

Now install $Q 9$ in the inner arc. 
qsa_par (1) = qsa_iron_ir

qsa_par (2) = qsa_iron_or

qsa_par $(3)=$ q9_length

nmed $=$ nmed_dx

call gsvolu ('Q9IF', 'TUBE', nmed, qsa_par, 3, ivolu)

call gsatt ('Q9IF', 'COLO', 6)

call gspos ('Q9IF', 1, 'HALL', q9_in_x_coord, q9_in_Y_coord, q9_in_z_coord, irot13, 'ONLY')

Install the collar inside the inner Q9.

qsa_par(1) = qsa_collar_ir

qsa_par $(2)$ = qsa_collar_or

nme $\bar{d}=$ nmed_g10

call gsvolu ('Q9IC', 'TUBE', nmed, qsa_par, 3, ivolu)

call gsatt ('Q9IC', 'COLO', 6)

call gsatt ('Q9IC', 'SEEN', 0 )

call gspos ('Q9IC', 1, 'Q9IF', $0.0 e 0,0.0 e 0,0.0 e 0,0$, 'ONLY')

$\mathrm{C}$

$\mathrm{C}$

$\mathrm{C}$

C

$\mathrm{C}$

C

$\mathrm{C}$

C

C

$\mathrm{C}$

$\mathrm{C}$

$\mathrm{C}$

$\mathrm{C}$

C

Now install the coil inside the inner 09 .

qsa_par(1) = qsa_coil_ir

qsa_par (2) = qsa_coil_or

nmed $=$ nmed_cu

call gsvolu ('Q9IL', 'TUBE', nmed, qsa_par, 3, ivolu)

call gsatt ('Q9IL', 'COLO', 6)

call gsatt ('Q9IL', 'SEEN', 0 )

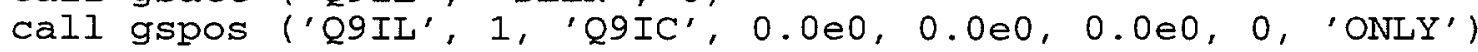

Install the gap between the beam tube and the coil.

qsa_par(1) = qsa_gap_ir

qsa_par $(2)$ = qsa_gap_or

nmed $=16$

call gsvolu ('Q9IG', 'TUBE', nmed, qsa_par, 3, ivolu)

call gsatt ('Q9IG', 'COLO', 6)

call gsatt ('Q9IG', 'SEEN', 0 )

call gspos ('Q9IG', 1 , 'Q9IL', $0.0 e 0,0.0 e 0,0.0 e 0,0$, 'ONLY')

Now install the beam tube inside the inner Q9.

qsa_par(1) = qsa_tube_ir

qsa_par (2) = qsa_tube_or

nme $\bar{d}=900$

call gsvolu ('Q9IT', 'TUBE', nmed, qsa_par, 3, ivolu)

call gsatt ('Q9IT', 'COLO', 6)

call gsatt ('Q9IT', 'SEEN', 0 )

call gspos ('Q9IT', 1, 'Q9IG', 0.0e0, $0.0 e 0,0.0 e 0,0$, 'ONLY')

Install the vacuum inside the tube.

qsa_par (1) $=0.0 \mathrm{e} 0$

qsa_par (2) = qsa_tube_ir

nme $\bar{d}=16$

call gsvolu ('Q9IV', 'TUBE', nmed, qsa_par, 3, ivolu)

call gsatt ('Q9IV', 'SEEN', 0)

call gspos ('Q9IV', 1, 'Q9IT', 0.0e0, $0.0 e 0,0.0 e 0,0$, 'ONLY')

Now install the outer $Q 9$.

qsa_par(1) = qsa_iron_ir

qsa_par (2) = qsa_iron_or

qsa_par (3) = q9_length

$n$ me $\bar{d}=$ nmed_dx 
C

call gsvolu ('Q9OF', 'TUBE', nmed, qsa_par, 3, ivolu)

call gsatt ('Q9OF', 'COLO', 6)

call gspos ('Q90F', 1, 'HALL', q9_out_x_coord, q9_out_y_coord, q9_out_z_coord, irot13, 'ONLY'

Install the collar inside the outer 09 .

qsa_par $(1)=$ qsa_collar_ir

qsa_par $(2)=$ qsa_collar_or

nmed $=$ nmed_g10

call gsvolu ('Q90C', 'TUBE', nmed, qsa_par, 3, ivolu)

call gsatt ('Q90C', 'COLO', 6)

cal1 gsatt ('Q90C', 'SEEN', 0)

call gspos ('Q90C', 1, 'Q9OF', 0.0e0, 0.0e0, 0.0e0, 0, 'ONLY')

Now Onstall the coil inside the outer 09 .

qsa_par (1) = qsa_coil_ir

qsa_par $(2)=$ qsa_coil_or

nmed $=$ nmed_cu

call gsvolu ('Q9oL', 'TUBE', nmed, qsa_par, 3, ivolu)

call gsatt ('Q9OL', 'COLO', 6)

call gsatt ('Q9OL', 'SEEN', 0)

call gspos ('Q9OL', 1, 'Q9OC', 0.0e0, $0.0 e 0,0.0 \mathrm{e}, 0,0$, 'ONLY')

Install the gap between the beam tube and the coil.

qsa_par (1) = qsa_gap_ir

qsa_par $(2)=$ qsa_gap_or

nme $\overline{\mathrm{d}}=16$

call gsvolu ('Q90G', 'TUBE', nmed, qsa_par, 3, ivolu)

call gsatt ('Q9OG', 'COLO', 6)

call gsatt ('Q9OG', 'SEEN', 0)

call gspos ('Q9OG', 1, 'Q9OL', 0.0e0, 0.0e0, 0.0e0, 0, 'ONLY')

Now install the beam tube inside the outer $Q 9$.

qsa_par (1) = qsa_tube_ir

qsa_par $(2)=$ qsa_tube_or

nmed $=900$

call gsvolu ('Q9oT', 'TuBE', nmed, qsa_par, 3, ivolu)

call gsatt ('Q9OT', 'COLO', 6)

call gsatt ('Q9OT', 'SEEN', 0 )

call gspos ('Q9OT', 1, 'Q9OG', 0.0e0, 0.0e0, 0.0e0, 0, 'ONLY')

Install the vacuum inside the tube.

qsa_par $(1)=0.0 \mathrm{e} 0$

qsa_par $(2)=$ qsa_tube_ir

nme $\bar{d}=16$

cal1 gsvolu ('Q90V', 'TUBE', nmed, qsa_par, 3, ivolu)

call gsatt ('Q9OV', 'SEEN', O)

call gspos ('Q9OV', 1, 'Q9OT', 0.0e0, 0.0e0, 0.0e0, 0, 'ONLY')

Install the magnets on the other side of the origin.

call gspos ('Q9IF',2, 'HALI', q9_in_x_coord, q9_in_y_coord,

$\$ \quad$-q9_in_z_coord, irot14, 'ONLY')

call gspos ('Q9OF', 2,' 'HALI', q9_out_x_coord, q9_out_y_coord, $\$$ -q9_out_z_coord, irot 14 , 'ONLY')

End installation of $Q 9$. Begin installation of $D 9$. d9_out_x_coord $=$ d8_out_x_coord + d8_a9.out_dist *

$\$$ save_this_sine

d9_out_z_coord $=$ d8_out_z_coord + d8_d9_out_dist * 
C

Now calculate the coordinates of the magnet in the inner arc.

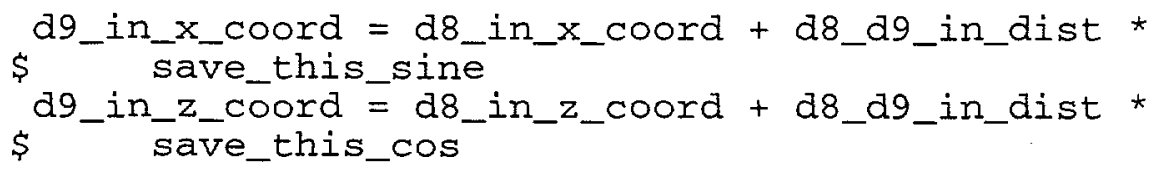

Now install the magnet in the outer arc.

d9_par $(1)=d 5$ _iron_ir

d9_par (2) = d5_iron_or

d9_par $(3)=$ d9_length

nmed = nmed_dx

call gsvolu ('D90F', 'TUBE', nmed, d9_par, 3, ivolu)

call gsatt ('D9OF', 'COLO', 6)

call gspos ('D90F', 1, 'HALL', d9_out_x_coord, d9_out_y_coord, $\$$ d9_out_z_coord, irot13, 'ONLY')

Install the collar inside the outer D9.

d9_par $(1)=$ d5_collar_ir

d9_par $(2)=$ d5_collar_or

nmed = nmed_g10

call gsvolu ('D90C', 'TUBE', nmed, d9_par, 3, ivolu)

call gsatt ('D9OC', 'COLO', 6)

call gsatt ('D90C', 'SEEN', 0)

call gspos ('D90C', 1, 'D9OF', 0.0e0, $0.0 e 0,0.0 e 0,0$, 'ONLY')

Now install the coil inside outer D9.

d9_par $(1)=$ d5_coil_ir

d9_par $(2)=$ d5_coil_or

nmed = nmed_cu

call gsvolu ('D9oL', 'TUBE', nmed, d9_par, 3, ivolu)

call gsatt ('D9OL', 'COLO', 6)

call gsatt ('D9OL', 'SEEN', O)

call gspos ('D90L', 1, 'D90C', 0.0e0, $0.0 \mathrm{e} 0,0.0 \mathrm{e} 0,0$, 'ONLY')

Install the gap between the beam tube and the coil in the outer magnet.

d9_par $(1)=$ d5_gap_ir

d9_par $(2)=$ d5_gap_or

nmed $=16$

call gsvolu ('D90G', 'TUBE', nmed, d9 par, 3, ivolu)

call gsatt ('D9OG', 'COLO', 6)

call gsatt ('D9OG', 'SEEN', 0)

call gspos ('D9OG', 1, 'D9OL', 0.0e0, 0.0e0, 0.0e0, 0, 'ONLY')

Now install the beam tube inside the outer D9.

d9_par (1) = d5_tube_ir

d9_par $(2)=$ d5_tube_or

nmed $=900$

call gsvolu ('D90T', 'TUBE', nmed, d9_par, 3, ivolu)

call gsatt ('D9OT', 'COLO', 6)

call gsatt ('D9OT', 'SEEN', 0)

call gspos ('D90T', 1, 'D9OG', $0.0 e 0,0.0 e 0,0.0 e 0,0$, 'ONLY')

Install the vacuum inside the tube.

d9 $\operatorname{par}(1)=0.0 \mathrm{e} 0$

d9_par (2) = d5_tube_ir

nmed $=16$ 
call gsvolu ('D9oV', 'TUBE', nmed, d9_par, 3, ivolu)

call gsatt ('D9OV', 'SEEN', 0)

call gspos ('D90V', 1, 'D90T', 0.0e0, 0.0e0, 0.0e0, 0, 'ONLY')

$\mathrm{C}$

C

Install the inner D9 magnets.

d9_par (1) = d5_iron_ir

d9_par $(2)=d 5$ _iron_or

d9_par $(3)=$ d9_length

nmed = nmed_dx

call gsvolu ('D9IF', 'TUBE', nmed, d9_par, 3, ivolu)

call gsatt ('D9IF', 'COLO', 6)

call gspos ('D9IF', 1, 'HALL', d9_in_x_coord, d9_in_y_coord, $\$$ d9_in_z_coord, irot13, 'ONLY')

Install the collar inside the inner D9.

d9_par $(1)=$ d5_collar_ir

d9_par $(2)=$ d5_collar_or

nmed = nmed $g 1 \overline{0}$

call gsvolu ('D9IC', 'TUBE', nmed, d9_par, 3, ivolu)

call gsatt ('D9IC', 'COLO', 6)

call gsatt ('D9IC', 'SEEN', 0)

call gspos ('D9IC', 1, 'D9IF', $0.0 e 0,0.0 e 0,0.0 e 0,0$, 'ONLY')

Now install the coil inside inner D9.

d9_par $(1)=$ d5_coil_ir

d9_par $(2)=$ d5_coil_or

nmed = nmed_cu

call gsvolu ('D9IL', 'TUBE', nmed, d9_par, 3, ivolu)

call gsatt ('D9IL', 'COLO', 6)

call gsatt ('D9IL', 'SEEN', 0 )

call gspos ('D9IL', 1, 'D9IC', $0.0 e 0,0.0 e 0,0.0 e 0,0$, 'ONLY')

Install the gap between the beam tube and the coil in the inner D9 magnet.

d9_par(1) = d5_gap_ir

d9_par $(2)=$ d5_gap_or

nmed $=16$

call gsvolu ('D9IG', 'TUBE', nmed, d9_par, 3, ivolu)

call gsatt ('D9IG', 'COLO', 6)

call gsatt ('D9IG', 'SEEN', 0)

call gspos ('D9IG', 1, 'D9IL', $0.0 e 0,0.0 e 0,0.0 e 0,0$, 'ONLY')

Now install the beam tube inside the inner D9.

d9_par $(1)=$ d5_tube_ir

d9_par (2) = d5_tube_or

nmed $=900$

call gsvolu ('D9IT', 'TUBE', nmed, d9_par, 3, ivolu)

call gsatt ('D9IT', 'COLO', 6)

call gsatt ('D9IT', 'SEEN', 0 )

call gspos ('D9IT', 1, 'D9IG', 0.0e0, 0.0e0, 0.0e0, 0, 'ONLY')

Install the vacuum inside the tube.

d9_par(1) $=0.0 e 0$

d9_par $(2)=$ d5_tube_ir

nmed $=16$

call gsvolu ('D9IV', 'TuBE', nmed, d9_par, 3, ivolu)

call gsatt ('D9IV',' 'SEEN', 0 )

call gspos ('D9IV', 1, 'D9IT', 0.0e0, $0.0 e 0,0.0 e 0,0$, 'ONLY')

Install the corresponding magnets on the other side of the origin. 
$\$$

call gspos ('D9IF',2, 'HALL', d9_in_x_coord, d9_in_y_coord, -d9_in_z_coord, irot14, 'ONLY')

\$ -d9_out_z_coord, irot14, 'ONLY') return

$\mathrm{C}$

C

Error branch points associated with phnx.par namelist file

continue

stop' RHIC_MAGNET_INSTALL <E>: No phnx.par file found' continue

stop ' RHIC_MAGNET_INSTALL <E>: Error in rhic_mag in phnx.par'

Miscellaneous notes of interest

I have not installed the vacuum in pipes that run between the magnets because the installation is tedious and the effect is small. At some later date this deficiency should be remedied.

Neither the stainless steel containment vessel nor the cryostat have been installed by this program. The installation of the containment vessels will be trivial, but I have no drawings of the cryostats at yet.

The color code for graphics output is located on page 369 of the GEANT manual in the section entitled "XINT002".

The allowed shapes in GEANT are specified on page 101 of the GEANT manual in the section entitled "GEOMO50".

The routine "gsvolu" is discussed on page 110 of the GEANT manual in the section entitled "GEOMI00".

The routine "gspos" is discussed on page 111 of the GEANT manual in the section entitled "GEOM100".

The definition of rotation matrices is discussed on page 124 of the GEANT manual in the section entitled "GEOM200". The name of the relevant subroutine is "gsrotm".

Particle definitions within the context of GEANT are found on pages 53 - 55 of the GEANT manual in the section entitled "CONS300".

Material definitions are found on page 39 of the GEANT manual in the section entitled "CONS100". The table presented therein is woefully small, and a larger table may be found in Appendix B, section 2 of the PISA/PISORP users' manual.

end 
Appendix B

the Fortran subroutine beam_gas_evt.f 
subroutine beam_gas_evt(ievstat)

implicit none

Beam gas event generator

Original version from Paul Kirk (LSU)

Calling variable

integer ievstat

Common block definition

include 'guevgen.inc'

Declare parameters that control dimensions of arrays below.

integer*4 no_theta_bins, no_bins_ptot, no_bins_pz

integer*4 no_cos_theta_bins, no_bins_pions, no_bins_nucleons

integer*4 no_bins_kaons, no_bins_gamma, no_bins_leptons

integer*4 no_bins_ptot_nucleon

$\mathrm{C}$

parameter (no_theta_bins = 36)

parameter (no_bins_ptot $=150$, no_bins_pz $=150$ )

parameter (no_cos_theta_bins $=40$, no_bins_pions $=100$ )

parameter (no_bins_nucleons $=50$, no_bins_kaons $=20$ )

parameter (no_bins_gamma $=20$, no_bins_leptons $=10$ )

parameter (no_bins_ptot_nucleon $=160$ )

$\mathrm{C}$

C

C

C

C

C

$\mathrm{C}$

$\mathrm{C}$

C

C

parameter (lun_hydrogen $=1151$, Iun_helium $=1152$ )

parameter (Iun_nitrogen $=1153$, Iun_output $=1150$ )

parameter (no_bins_z_plot $=100$ )

CFM I/O additions

integer istat

integer lun_fnames

parameter (Iun_fnames $=1199$ )

character $* 80$ evtname (3)

character $* 80$ outname

Declare real variables below.

real*4 btot, phib, trig_factor_1, trig_factor_2

real*4 trig_factor_3, trig_factor_4, trig_factor_5

integer*4 ievent, igeant

integer*4 lun_hydrogen, lun_helium, lun_nitrogen

integer*4 lun_output, no_open_files, lun_input

integer*4 no_bins_z_plot, option_number

integer*4 number_nitrogen_int, logical_unit

integer*4 idtemp, mxtot_temp
! error return from KUOPEN

! file for file names

! names of the event files ( $\mathrm{H}, \mathrm{He}$, and $\mathrm{N}$ )

! name for output file

ing

PNk as part of the modifications that were necessary to suppress 
real*4 theta_low, theta_high

real*4 upper_lim_ptot, upper_lim_pz, lower_lim_ptot

real*4 lower_lim_pz, bin_width_ptot, bin_width_pz

real*4 no_pi_plus (no_bins_pions)

real*4 no_pi_minus (no_bins_pions)

real*4 no_pi_naught (no_bins_pions), no_neutrons (no_bins_nucleons)

real*4 no_protons (no_bins_nucleons)

real*4 no_gamma (no_bins_gamma)

real*4 no_electron (no_bins_leptons), no_positron(no_bins_leptons)

real*4 no_kaon_plus (no_bins_kaons)

real*4 no_kaon_minus (no_bins_kaons), no_kaon_0 (no_bins_kaons)

real*4 no_pi_plus_theta (no_theta_bins), no_pi_plus_phi (36)

real*4 no_pi_plus_cos_theta (no_cos_theta_bins)

real*4 no_pi_plus_ptot (no_bins_ptot)

real*4 no_pi_plus_pz (no_bins_pz)

real*4 no_pi_minus_theta (no_theta_bins), no_pi_minus_phi (36)

real*4 no_pi_minus_cos_theta (no_cos_theta_bins)

real*4 no_pi_minus_ptot (no_bins_ptot)

real*4 no_pi_minus_pz (no_bins_pz)

real*4 no_pi_naught_theta (no_theta_bins), no_pi_naught_phi

real*4 no_pi_naught_cos_theta (no_cos_theta_bins)

real*4 no_pi_naught_ptot (no_bins_ptot)

real*4 no_pi_naught_pz (no_bins_pz)

real*4 no_ka_plus_theta (no_theta_bins), no_ka_plus_phi (36)

real*4 no_ka_plus_cos_theta (no_cos_theta_bins)

real*4 no_ka_plus_ptot (no_bins_ptot)

real*4 no_ka_plus_pz (no_bins_pz)

real*4 no_ka_minus_theta (no_theta_bins), no_ka_minus_phi

real*4 no_ka_minus_cos_theta (no_cos_theta_bins)

real*4 no_ka_minus_ptot (no_bins_ptot)

real*4 no_ka_minus_pz (no_bins_pz)

real*4 no_ka_naught_theta (no_theta_bins), no_ka_naught_phi

real*4 no_ka_naught_cos_theta (no_cos_theta_bins)

real*4 no_ka_naught_ptot (no_bins_ptot)

real*4 no_ka_naught_pz (no_bins_pz)

real*4 no_protons_theta (no_theta_bins), no_protons_phi (36)

real*4 no_protons_cos_theta (no_cos_theta_bins)

real*4 no_protons_ptot (no_bins_ptot_nucleon)

real*4 no_protons_pz (no_bins_ptot_nucleon)

real*4 no_neutrons_theta (no_theta_bins), no_neutrons_phi

real*4 no_neutrons_cos_theta (no_cos_theta_bins)

real*4 no_neutrons_ptot (no_bins_ptot_nucleon)

real*4 no_neutrons_pz (no_bins_ptot_nucleon)

real*4 no_gammas_theta (no_theta_bins), no_gammas_phi (36)

real*4 no_gammas_cos_theta (no_cos_theta_bins)

real*4 no_gammas_ptot (no_bins_ptot)

real*4 no_gammas_pz (no_bins_pz)

real*4 no_electrons_theta (no_theta_bins), no_electrons_phi

real*4 no_electrons_cos_theta (no_cos_theta_bins)

real*4 no_electrons_ptot (no_bins_ptot)

real*4 no_electrons_pz (no_bins_pz)

real*4 no_positrons_theta (no_theta_bins), no_positrons_phi

real*4 no_positrons_cos_theta (no_cos_theta_bins)

real*4 no_positrons_ptot (no_bins_ptot)

real*4 no_positrons_pz (no_bins_pz)

\section{Following "number" variables were originally reals}

integer number_positive_pions, number_negative_pions

integer number_neutral_pions, number_protons, number_neutrons

integer number_positive_kaons, number_negative_kaons

integer number_neutral_kaons, number_gammas

integer number_positrons, number_electrons

real*4 probability_hydrogen, probability_helium

real*4 probability_nitrogen, total_probability

real*4 rel_prob_hyd̆rogen, rel_prob_helium, rel_prob_nitrogen 
real*4 hydrogen_thickness (4), helium_thickness (4) real*4 nitrogen_thickness (4), length_region(4)

real*4 total_hyd̄rogen_thickness, total_helium_thickness

real*4 total_nitrogen_thickness, total_length_hydrogen

real*4 total_length_helium, total_length_nitrogen

real*4 random_number, distance, cuts(4), sum, numerator

rea $1 * 4$ denominator, least_z_region (4), intercept_line_1

real*4 slope_line_1, intercept_line_2, slope_line_2

real*4 intercept_Iine_3, slope_line_3

real*4 intercept_line_4, slope_line_4

real*4 intercept_line_5, slope_line_5

real*4 lower_lim_z_plot, upper_lim_z_plot, bin_width_z_plot

real*4 z_plot_hyōrogen(100), z_plot_helium (100)

real*4 z_plot_nitrogen (100)

real*4 change_sign_x, change_sign_z, z_in_meters

real*4 x_coordinate_interaction, $z$ _coordinate_interaction

real* $4 \mathrm{px}, \mathrm{py}, \mathrm{pz}$

real*4 azimuthal_angle, zenith_angle, cosine_of_angle

real*4 temp, temp 01 , total_momentum, temp_x, temp_z

real*4 sigma_au_hydrogen, sigma_au_helium, sigma_au_nitrogen

real*4 test_value, delta_z, rndm, theta_bin_width

real* 4 total_length

real * 4 x_coordinate_valve_1, z_coordinate_valve_1

real*4 x_coordinate_valve_2, z_coordinate_valve_2

real*4 x_coordinate_valve_3, z_coordinate_valve_3

parameter (x_coordinate_valve_t $=-1.443 \bar{e}+01$ )

parameter (x_coordinate_valve_2 $=-2.57025 e+01$ )

parameter (x_coordinate_valve_3 $=-3.84831 e+01$ )

parameter (z_coordinate_valve_1 $=1.930 e+03$ )

parameter (z_coordinate_valve_2 $=3.75778 e+03$ )

$\mathrm{C}$

parameter (z_coordinate_valve_3 $=7.23592 \mathrm{e}+03$ )

real*4 length_r4_segment_1, length_r4_segment_2

real*4 length_r4_segment_3, length_r4_segment_4

parameter (length_r4_segment_1 = 2.20971e+03)

parameter (length_r4_segment_2 = 1.51893e+03)

parameter (length_r4_segment_3 $=2.94554 \mathrm{e}+03$ )

C

parameter (length_r4_segment_4 $=1.48212 e+03$ )

real*4 x_coord_do, z_coord_do

real *4 x_coord_d5_end, z_coord_d5_end

real * 4 x_coord_d6_end, z_coord_d6_end

real * 4 x_coord_d8_end, z_coord_d8_end

parameter (x_coord_do $=-2.008 \overline{8} 5 e+01$ )

parameter ( $z$ _coord_do $=2.22998 e+03$ )

parameter (x_coord_d5_end $=-4.66028 e+01$ )

parameter ( $z_{\text {_coord_d5_end }}=9.44562 \mathrm{e}+03$ )

parameter (x_coord_d6_end $=2.28202 \mathrm{e}$ )

parameter (z_coord_d6_end $=1.09638 \mathrm{e}+04)$

parameter (x_coord_d8_end $=1.32873 e+02$ )

C

parameter (z_coord_d8_end $=1.39064 \mathrm{e}+04$ )

logical*4 region_1_segment_1, region_1_segment_2

logical*4 region_2_segment_1, region_2_segment_2

logical*4 region_3

logical*4 region_4_segment_1, region_4_segment_2

logical*4 region_4_segment_3, region_4_segment_4

real*4 angle_zz_r1_s1, angle_zz_r1_s2

real*4 angle_zz_r2_s1, angle_zz_r2_s2

real*4 angle_zz_r3, keep_this_angle

real*4 angle_zz_r4_s1, angle_zz_r4_s2

real*4 angle_zz_r4_s3, angle_zz_r4_s4

parameter (angle_zz_ri_s1 = $\overline{1} .8 \overline{0} e+\overline{0} 2$ )

parameter (angle_zz_r1_s2 $=1.78919 e+02$ )

parameter (angle_zz_r2_s1 $=1.78919 e+02$ ) 
parameter (angle_zz_r2_s2 = $1.79789 e+02$ )

parameter (angle_zz_r3 $=1.79789 e+02$ )

parameter (angle_zz_r4_s1 $=1.79789 e+02$ )

parameter (angle_zz_r4_s2 $=1.81844 \mathrm{e}+02$ )

parameter (angle_zz_r4_s3 $=1.82541 e+02$ )

parameter (angle_zz_r4_s4 $=1.84771 e+02$ )

The following real variables were added on september 21, 1995

by PNK as part of the attempt to eliminate "spectator" nucleons.

real*4 p1_temp, p2_temp, p3_temp, p4_temp

parameter (theta_low $=0.0 \mathrm{e}$, theta_high $=1.80 \mathrm{e}+02$ )

parameter (upper_lim_ptot $=1.50 e+02$, upper_lim_pz $=1.50 e+02$ )

parameter (lower_lim_ptot $=0.0 e 0$, lower_lim_pz $=0.0 e 0$ )

parameter (bin_width_ptot $=1.0 e 0$, bin_width_pz $=1.0 e 0$ )

parameter (theta_bin_width $=5.0 e 0$ )

parameter (lower_lim_z_plot $=0.0 e 0$, upper_lim_z_plot $=2.0 e+02$ )

parameter (bin_width_z_plot $=2.0 \mathrm{e}$ )

parameter (intercept_line_1 $=2.19756 \mathrm{e}+01$ )

parameter (intercept_line_2 $=-1.18943 e+01$ )

parameter (intercept_line_3 $=-3.50756 e+02$ )

parameter (intercept_line_4 $=-4.84280 e+02$ )

$\mathrm{C}$

parameter (intercept_line_5 $=-1.02785 e+03$ )

The slopes shown below are appropriate for the outer arc only!

parameter (slope_line_1 $=-1.88630 \mathrm{e}-02$ )

parameter (slope_line_2 $=-3.67456 \mathrm{e}-03$ )

parameter (slope_line_3 $=3.22004 \mathrm{e}-02$ )

parameter (slope_line_4 $=4.43790 \mathrm{e}-02$ )

parameter (slope_line_5 $=8.34669 \mathrm{e}-02$ )

parameter (change_sign_x $=1.0 e 0$, change_sign_z $=1.0 \mathrm{e} 0$ )

parameter (sigma_au_hydrogen $=2.10 \mathrm{e}-24$ )

parameter (sigma_au_helium $=2.48 \mathrm{e}-24$ )

parameter (sigma_au_nitrogen $=3.06 \mathrm{e}-24$ )

logical*4 first_access, first_enabled_region

logical*4 hydrogen_file, helium_file, nitrogen_file

logical*4 enable_region(4), true_distributions

logical*4 hydrogen_interaction, helium_interaction

logical*4 nitrogen_interaction

$\mathrm{C}$

C

C

Declare character variables below.

CHARACTER * 80 CARTM

CHARACTER $* 9$ LBL

CHARACTER * 10 CAR1

CHARACTER *19 CAR2

CHARACTER ${ }^{\star} 6$ CAR3
$\mathrm{C}$

C

c

C

C

C

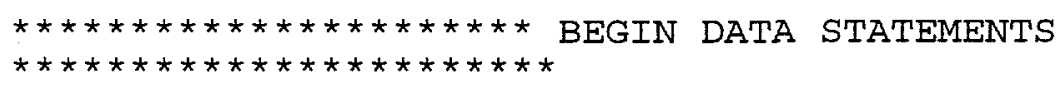

data first_access /.true./

data helium_thickness / 0.0e0,2.1e+09, 0.0e0, 9.4e+09/

data hydrogen_thickness / $6.2 e+10,8.0 e+10,1.1 e+11,3.6 e+11 /$

data nitrogen_thickness / 4.7e+09, 0.0e0, 8.4e+09, 0.0e0/

data least_z_region / 0.0e0, 1.930e+03, 3.75778e+03,7.23592e+03/

data enable_region / .false., false., true., false./

data length_region $/ 1.93014 e+03,1.82781 e+03,3.47816 e+03$,

$\$$

$$
8.1563 \bar{e}+03 /
$$


$* * * * * * * * * * *$ BEGIN DEFINITIONS OF LOCALLY CREATED VARIABLES
$* * * * * * * * *$

CARTM is an 80 byte array of characters that is used for reading in Ascii text from the header of the HIJET file.

CAR1, CAR2, and CAR3 are also arrays of characters that serve no purpose other than reading in ascii text from the first line of each event.

First_access is a logical variable that is set equal to .true. in a

data statement. Upon first call to this routine the file that contains the hijet file is set opened, the header to the file is read

in, and the value of first_access itself is set equal to . false.

IEVENT is the number of the events in the HIJET file. The files to which I currently have access contains 100 events.

LBL is a character in which is stored ascii text that identifies the

particle. For example, "PIO" is the label for a neutral pion.

This

variable plays no role whatever in the logic.

NPT is the number of particles that were produced in the HIJET event under examination.

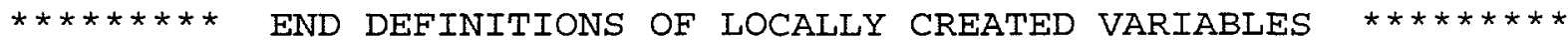

$\star * * * * * * * * * * * * * * * * *$ DESCRIPTION OF THE HIJET FILES

The HIJET files to which I currently have access comprise 100 interactions between Au of full energy and stationary atoms. The files are located in /diskl/kirk. The names of the files to which I currently have access are auhebgas.evt, which comprises 100 interactions between $\mathrm{Au}$ and $\mathrm{He}$; aunbgas.evt, which comprises 100 interactions between $\mathrm{Au}$ and nitrogen; and aupbgas .evt, which comprises 100 interactions between $\mathrm{Au}$ and hydrogen. These files were produced by RON LONGACRE at BNL.

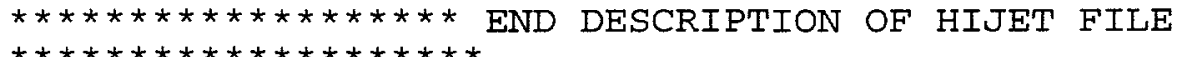

if (first_access) then

first_access $=$. false.

trig_factor_1 $=\operatorname{sgrt}(1.000+$ slope_line_l $* * 2)$

trig_factor_2 $=$ sqrt $(1.0 e 0+$ slope_line_2 $* * 2)$

trig_factor_3 $=\operatorname{sqrt}(1.0$ e $0+$ slope_line_3 $* * 2)$

trig_factor_4 $=$ sqrt $(1.000+$ slope_line_4 $* * 2)$

trig_factor_5 $=\operatorname{sqrt}(1.000+$ slope_line_5 $* * 2)$

multi_xyz $=0$

if (kevt_par (1).gt.0.5) then

else

hydrogen_file $=$.true.

hydrogen_file $=$.false. 
endif

if (kevt_par (2).gt.0.5) then

else

helium_file = .true.

endif

helium_file = .false.

if (kevt_par (3).gt.0.5) then

else

nitrogen_file $=$.true.

endif

nitrogen_file $=$.false.

if (kevt_par (4).gt.0.5) then else

true_distributions $=$.true.

endif

true_distributions $=$. false.

$\mathrm{C}$

$\mathrm{C}$

C

c

$\mathrm{C}$

C

C

C

C

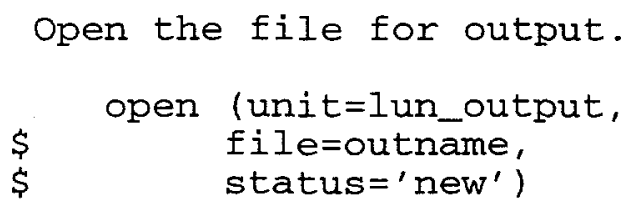

file does not exist, so use default names

evtname $(1)=$ '/disk1/kirk/aupbgas.evt'

evtname $(2)=' / \mathrm{disk1} / \mathrm{kirk} /$ auhebgas.evt'

evtname $(3)=$ '/disk1/kirk/aunbgas.evt'

outname =

endif

'/disk1/kirk/OUTPUT/output_file.text'

Open the desired files for input and count them. This programs accommodates from one to three files of HIJET data.

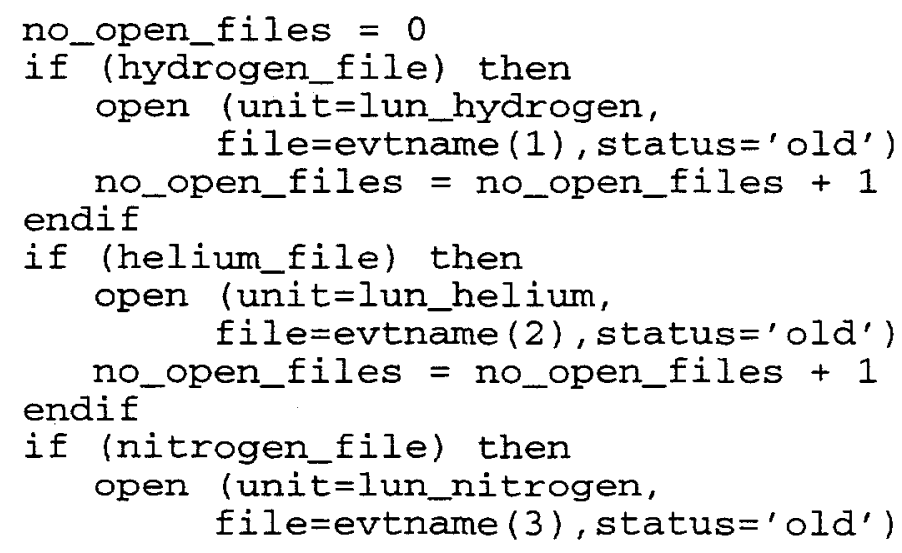



endif

no_open_files $=$ no_open_files +1

Determine which element, or combination of elements, the user has selected as targets. There are seven possible combinations of elements, and we now set the value of the variable "option_number" according to the combination selected by the user. The key is shown below.

Hydrogen Helium Nitrogen option number

$\begin{array}{llll}\mathrm{Y} & \mathrm{N} & \mathrm{N} & 1 \\ \mathrm{~N} & \mathrm{Y} & \mathrm{N} & 2 \\ \mathrm{~N} & \mathrm{~N} & \mathrm{Y} & 3 \\ \mathrm{Y} & \mathrm{Y} & \mathrm{N} & 4 \\ \mathrm{Y} & \mathrm{N} & \mathrm{Y} & 5 \\ \mathrm{~N} & \mathrm{Y} & \mathrm{Y} & 6 \\ \mathrm{Y} & \mathrm{Y} & \mathrm{Y} & 7\end{array}$

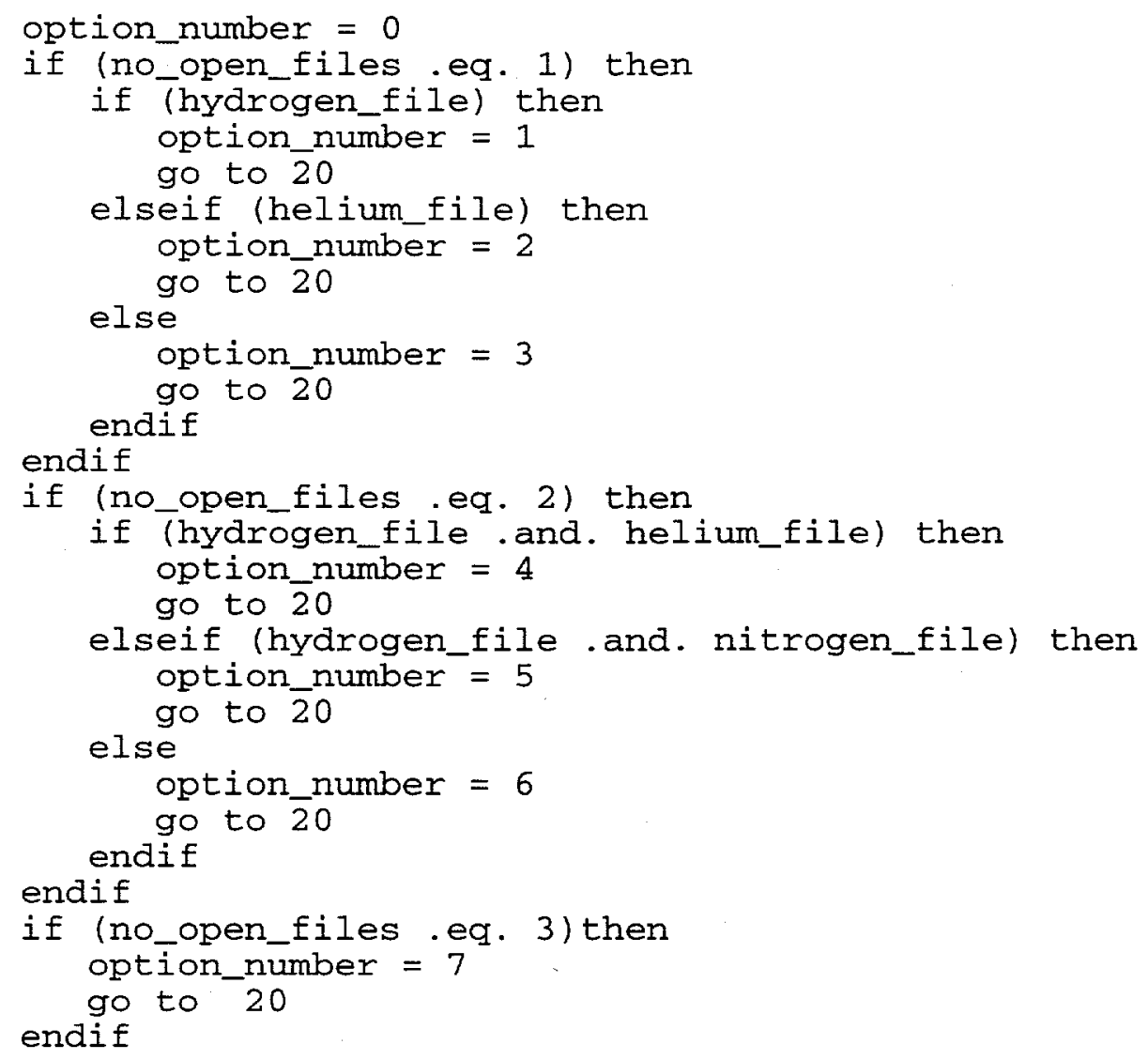

There is no way for the user to reach this point unless he failed to open at least one file.

write (1un_output,600)

goto 17

Ascertain which sections of the beampipe the user wants to activate, and calculate the total thicknesses of the target elements

if the users has selected to use the actual profiles.

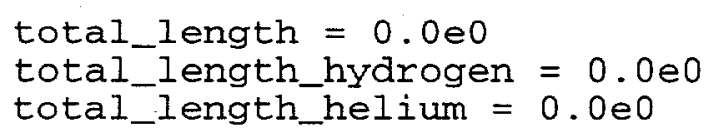


total_length_nitrogen $=0.0 e 0$

total_hydrogen_thickness $=0.0 e 0$

total_helium_thickness $=0.0$ e0

total_nitrogen_thickness $=0.0 e 0$

do $21 i=1,4$

if (enable_region(i)) then

total_length = total_length + length_region(i)

if (hydrogen_file) then

total_length_hydrogen = total_length_hydrogen +

$\$$

$\$$

$\$$

$\$$

$\$$

$\$$

$\$$

$\$$

21

C

C

C

C

$\$$

endif

endif

endif

$\$$

if (true_distributions) then total_hydrogen_thickness = total_hydrogen_thickness endif

endif

if (helium_file) then

if (true_distributions) then

total_helium_thickness = total_helium_thickness + helium_thickness (i)

if ( $i$.eq. $\overline{2}$.or. $i$.eq. 4) then

total_length_helium = total_length_helium +

endif length_region(i)

else

total_length_helium = total_length_helium +

endif

length_region (i)

endif

if (nitrogen_file) then

if (true_distributions) then

total_nitrogen_thickness = total_nitrogen_thickness

+ nitrogen_thickness(i)

if ( $i$.eq. 1 .or. $i$.eq. 3) then

total_length_nitrogen = total_length_nitrogen + endif length_region(i)

else

total_length_nitrogen $=$ total_length_nitrogen +

endif

length_region (i)

endif

endif

continue

Check for possible blunders on the part of the user that would create havoc later on.

if (total_length .eq. 0.0 e0) then

write (Iun_output, 601)

goto 17

if (helium_file and. true_distributions) then

if (.not. enable_region (2) and. not. enable_region(4)) then

write (lun_output, 602)

endif goto. 17

if (nitrogen_file and. true_distributions) then

if (.not. enable_region ( 1 ) . and. not. enable_region(3)) then

write (lun_output, 603)

goto 17

endif 
probability_hydrogen $=0.0 e 0$

probability_helium $=0.0 e 0$

probability_nitrogen $=0.0 e 0$

if (hydrogen_file) then

if (true distributions) then

$\$$

probability_hydrogen = total_hydrogen_thickness * sigma_au_hydrogen

else endif

probability_hydrogen = sigma_au_hydrogen

endif

if (helium_file) then

if (true_distributions) then

$\$$ probability_helium = total_helium_thickness *

Calculate the relative probabilities for interactions with the chosen targets.

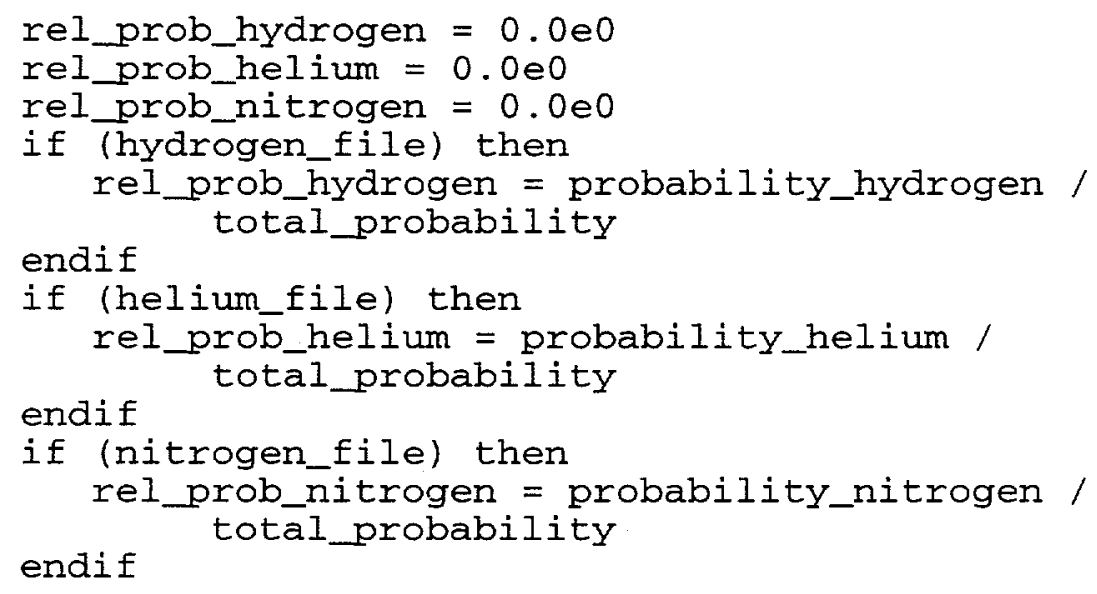

Read in the header from the HIJET file. The header is shown below

$3 / 8 / 95 \quad 15: 02: 06$

AU $->>\mathrm{HE} \quad 13.770$

MASSES, CHARGES $=197.000 \quad 79.000 \quad 4.000 \quad 2.000$ CENT $=1.000$

BEAM RADIUS 6.299 TARGET RADIUS 1.307 ECMMIN 3.000

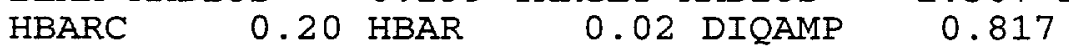

if (hydrogen_file) then logical_unit = lun_hydrogen READ (logical_unit, 1101 ) cartm write (Iun_output,1102) cartm 
READ (logical_unit, 1101) cartm write (Iun_output, 1102) cartm READ (logical_unit, 1101) cartm write (lun_output,1102) cartm READ (logical_unit, 1101) cartm write (Iun_output,1102) cartm READ (logical_unit,1101) cartm endif write (Iun_output,1102) cartm

if (helium_file) then

logical_unit = lun helium READ (logical_unit, 1101) cartm write (1un_output,1102) cartm READ (logical_unit, 1101) cartm write (lun_output, 1102) cartm READ (logical_unit, 1101) cartm write (lun_output,1102) cartm READ (logical_unit, 1101) cartm write (lun_output,1102) cartm READ (logicall_unit, 1101) cartm endif write (Iun_output,1102) cartm

if (nitrogen_file) then logical_unit = lun_nitrogen READ (logical_unit, 1101 ) cartm write (lun_output,1102) cartm READ (logical_unit, 1101) cartm write (lun_oütput, 1102) cartm READ (logical_unit, 1101) cartm write (lun_output,1102) cartm READ (logical_unit, 1101) cartm write (1un_output, 1102) cartm READ (logical_unit, 1101) cartm endif write (lun_output,1102) cartm

C C $\mathrm{C}$

200

2011
Clear arrays and variables.

do $200 i=1$, no_theta_bins no_pi_plus_theta(i) $=0.000$ no_pi_minus_theta $(i)=0.0 e 0$ no_pi_naught_theta $(i)=0.0 e 0$ no_ka_plus_theta $(i)=0.0 \mathrm{e}$ no_ka_minus_theta $(i)=0.0 e 0$ no_ka_naught_theta $(i)=0.0 e 0$ no_protons_theta $(i)=0.0 \mathrm{e} 0$ no_neutrons_theta $(i)=0.0 e 0$ no_gammas_theta $(i)=0.0 e 0$ no_electrons_theta $(i)=0.0 e 0$ no_positrons_theta $(i)=0.0 e 0$ continue

do $2011 i=1$, no_bins_pz

no_pi_plus_pz $(\bar{i})=0.0 e 0$ no_pi_minus_pz $(i)=0.0 e 0$ no_pi_naught_pz $(i)=0.0 \mathrm{e} 0$ no_ka_plus_pz (i) $=0.0 e 0$ no_ka_minus_pz (i) $=0.0 e 0$ no_ka_naught_pz(i) $=0.0 \mathrm{e}$ no_gammas_pz(i) $=0.0 e 0$ no_electrons_pz(i) $=0.0 e 0$ no_positrons_pz $(i)=0.0 e 0$ continue

do $2012 i=1$, no_bins_ptot no_pi_plus_ptot $(i)=0.000$ no_pi_minus_ptot $(i)=0.000$ 
no_pi_naught_ptot $(i)=0.0 e 0$

no_ka_plus_ptot $(i)=0.0 \mathrm{e} 0$

no_ka_minus_ptot $(i)=0.0 e 0$

no_ka_naught_ptot $(i)=0.000$

no_gammas_ptot $(i)=0.0 e 0$

no_electrons_ptot $(i)=0.0 e 0$

no_positrons_ptot $(i)=0.0 e 0$

continue

do $2222 i=1$, no_bins_ptot_nucleon

no_protons_ptot $(i)=0.0 \mathrm{e} 0$

no_neutrons_ptot $(i)=0.0 e 0$

no_protons_pz $(i)=0.0 e 0$

no_neutrons_pz $(i)=0.0 e 0$

2222

continue

do $2013 i=1,36$

no_pi_plus_phi $(i)=0.0 e 0$

no_pi_minus_phi(i) $=0.0 e 0$

no_pi_naught phi(i) $=0.0 \mathrm{e}$

no_ka_plus_phi $(i)=0.0 \mathrm{e} 0$

no_ka_minus_phi(i) $=0.000$

no_ka_naught_phi(i) $=0.0 \mathrm{e} 0$

no_protons_phi(i) $=0.0 \mathrm{e}$

no_neutrons_phi(i) $=0.0 \mathrm{e}$

no_gammas_phi $(i)=0.0 \mathrm{e} 0$

no_electrons_phi(i) $=0.0 e 0$

2013

no_positrons_phi(i) $=0.0 e 0$

continue

do $2014 i=1$, no_cos_theta_bins

no_pi_plus_cos_theta(i) $=0.000$

no_pi_minus_cos_theta $(i)=0.0 e 0$

no_pi_naught_cos_theta $(i)=0.0 e 0$

no_ka_plus_cos_theta $(i)=0.0 e 0$

no_ka_minus_cos_theta $(i)=0.0 e 0$

no_ka_naught_cos_theta(i) $=0.000$

no_protons_cos_theta $(i)=0.0 e 0$

no_neutrons_cos_theta $(i)=0.0 \mathrm{e} 0$

no_gammas_cos_theta $(i)=0.0 e 0$

no_electrons_cos_theta $(i)=0.0 e 0$

no_positrons_cos_theta $(i)=0.0 e 0$

continue

do $205 i=1$, no_bins_pions

no_pi_plus $(i)=0.0 \mathrm{e} 0$

no_pi_minus $(i)=0.0 e 0$

205

no_pi_naught $(i)=0.0 e 0$

continue

do $206 i=1$, no_bins_nucleons

no_neutrons $(i \overline{)}=0.0 e 0$

206

no protons $(i)=0.0 \mathrm{e} 0$

continue

do $207 i=1$, no_bins_kaons

no_kaon plus $(\bar{i})=\overline{0} .0 e 0$

no_kaon_minus $(i)=0.0 e 0$

no_kaon_o $(i)=0.0 e 0$

207 continue

do $208 i=1$, no_bins_gamma

no_gamma $(i)=0.0 e^{0}$

208

continue

do $209 i=1$, no_bins_leptons

no_electron $(i \overline{)}=0.0$ e0

no_positron $(i)=0.0 e 0$

209 continue

number_hydrogen_int $=0$

number_helium_int $=0$

number_nitrogen_int $=0$

do $222 \overline{3} i=1, \overline{1} 00$

z_plot_hydrogen $(i)=0.0 e 0$ 

endif

$$
\text { z_plot_helium }(i)=0.0 e 0
$$

$$
z \text { plot_nitrogen }(i)=0.0 e 0
$$

C

C first call to this routine. Begin the code that executed upon every call to the routine.

Some variables must be cleared at the beginning of each new event.

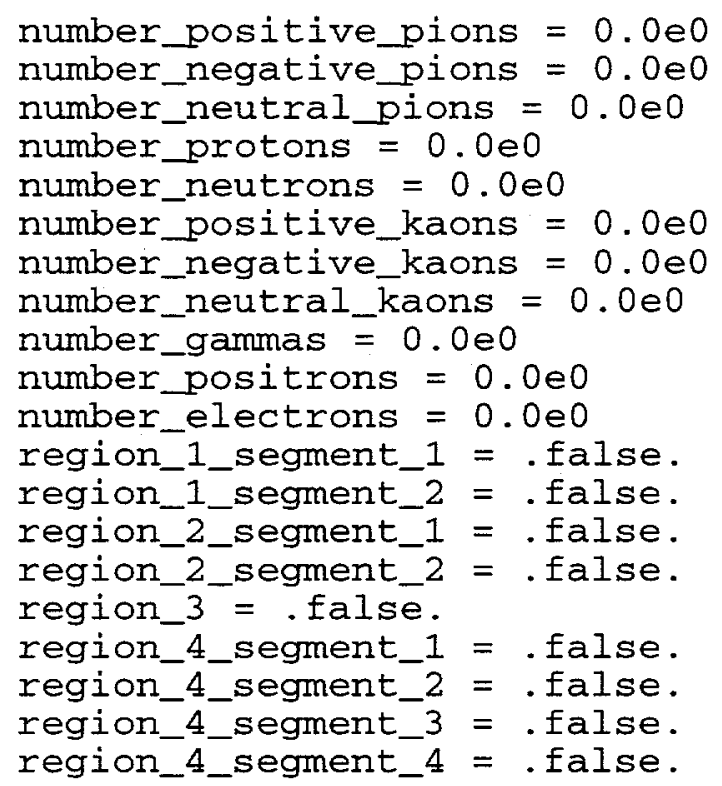


else

goto 25

else

nitrogen_interaction $=$.true.

endif

goto 25

if (random_number. Ie. rel_prob_helium) then

helium_interaction $=$.true.

else goto $2 \overline{5}$

endif

nitrogen_interaction $=$.true.

endif goto 25

endif

if (no_open_files .eq. 3) then

if (random_number.le. rel_prob_hydrogen) then hydrogen_interaction $=$.true. goto 25

$\$$

elseif (random_number.1e. (rel_prob_hydrogen +

$\mathrm{C}$

C

$\mathrm{C}$

25

C

C

$\mathrm{C}$

C

$\mathrm{C}$

$\mathrm{C}$

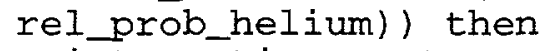

In principle the following statement should never be executed.

write (Iun_output, 604)

goto 17

continue

Locate the distance between the origin and the position of the interaction. In this context "distance" means distance as measured

along enabled regions only!

do $30 i=1,4$

$$
\operatorname{cuts}(i)=-1.0 e 0
$$

continue

sum $=0.0 e 0$

numerator $=0.0 e 0$

denominator $=0.0 \mathrm{e} 0$

if (hydrogen_interaction) then

denominator = total_hydrogen_thickness goto 29

elseif (helium_interaction) then

denominator = total_helium_thickness

else goto 29

endif

denominator $=$ total_nitrogen_thickness

continue

random_number $=r$ Icran $(0)$

random_number $=\operatorname{rndm}()$

if (hydrogen_interaction) then

if (true_distributions) then

do $31 \overline{0} i=1,4$

if (enable_region(i)) then

numerator = numerator + hydrogen_thickness $(i)$

endif cuts $(i)$ = numerator $/$ denominator

continue 
first_enabled_region $=$.true.

do $31 \overline{1} i=1, \overline{4}$

C

C

C

C

7613

311

C

C

C

312

C

C

C

C

Note that the following inequality can never be satisfied if region $i$ is not enabled.

$\$$

if (random_number.le. cuts(i)) then interaction_region $=i$ if (first_enabled_region) then delta_ $\bar{z}=$ (random_number $/ \operatorname{cuts}(i))$ * else length_region(i)

temp $=-1.0 e 0$

do $7613 \mathrm{k}=1, i-1$

if (cuts (k) .gt. temp) then endif temp $=\operatorname{cuts}(\mathrm{k})$

continue

temp01 = $\operatorname{cuts}(i)-$ temp delta_z $=(($ random_number - temp $) /$ temp01) * $\$$ endif length_region (i) goto 28

elseif (first_enabled_region) then if (cuts(i) .gt. $0.0 \mathrm{e}$ ) then first_enabled_region $=$.false.

endif endif

continue else

Arrive here if user wants uniform densities.

distance $=$ random_number $*$ total_length_hydrogen

do $312 i=1,4$

if (enable_region(i)) then test_value = distance - length_region (i)

if (test_value .gt. 0.0e0) then distance = distance - length_region (i) else delta_z = distance interaction_region $=i$ endif goto 28

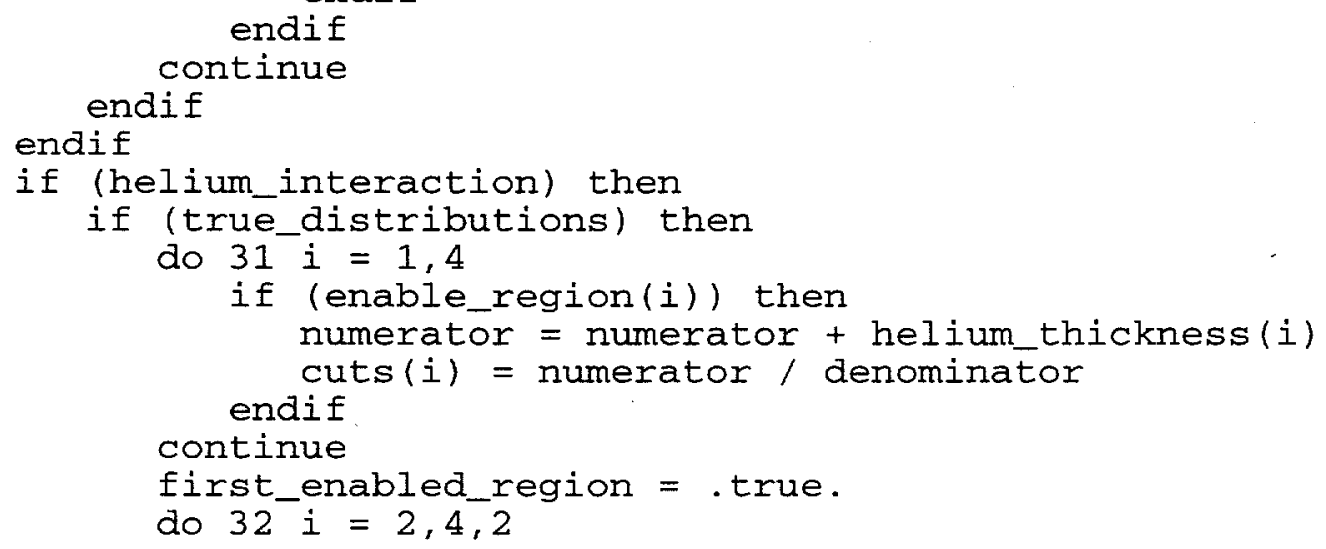

endif

Note that the following inequality can never be satisfied if region $i$ is not enabled.

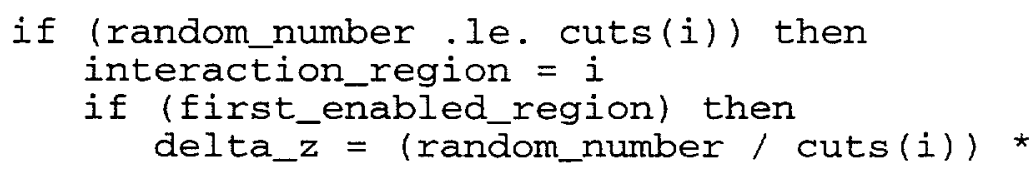


else

$$
\text { temp }=-1.0 e 0
$$

do $7614 \mathrm{k}=1$, $\mathrm{i}-1$

if (cuts (k) .gt. temp) then

endif

temp $=\operatorname{cuts}(k)$

continue

temp01 = cuts $(i)-$ temp

delta_z $=(($ random_number - temp $) /$ temp01 $)$ *

$\$$

length_region (i)

endif

goto 28

elseif (first_enabled_region) then

if (cuts (i) .gt. $0.0 \mathrm{e} 0$ ) then endif

first_enabled_region $=$.false.

endif$$
\text { ontinue }
$$

else

$C$
$C$
$C$

Arrive here if user wants uniform densities.

distance $=$ random_number * total_length_helium

do $33 i=1,4$

if (enable_region(i)) then

test_value = distance - length_region (i)

if (test_value gt. $0.0 e 0$ ) then

else

distance = distance - length_region (i)

endif

33

delta_z = distance

interaction_region $=i$

endif goto 28

continue

endif

endif

if (nitrogen_interaction) then

if (true_distributions) then

do $31 \overline{9} i=1,4$

if (enable_region(i)) then

numerator = numerator + nitrogen_thickness $(i)$

endif cuts $(i)=$ numerator / denominator

319

continue

first_enabled_region $=$.true. do $32 \overline{1} i=1, \overline{3}, 2$

$\mathrm{C}$

$\mathrm{C}$

Note that the following inequality can never be satisfied if region $i$ is not enabled.

if (random_number.1e. cuts(i)) then

interaction_region $=i$

if (first_enabled_region) then

$\$$ delta_z $=($ random_number $/ \operatorname{cuts}(i))$ *

else length_region(i)

temp $=-1.0 e 0$

do $7615 \mathrm{k}=1, i-1$

if (cuts $(k)$.gt. temp) then

endif

temp $=\operatorname{cuts}(\mathrm{k})$

7615

continue

temp01 = cuts $(i)-$ temp

delta_z $=(($ random_number - temp $) /$ temp01) * 
endif

length_region (i)

goto 28

elseif (first_enabled_region) then

if (cuts(i).gt. 0.0e0) then

endif

first_enabled_region = .false.

321 endif

continue

C else

Arrive here if user wants uniform densities.

distance $=$ random_number $*$ total_length_nitrogen

do $333 i=1,4$

if (enable_region(i)) then

test_value = distance - length_region (i)

if (test_value .gt. 0.0e0) then else distance = distance - length_region (i)

333 endif delta_z = distance interaction_region $=i$ goto 28

endif

continue

endif

endif

C

$\mathrm{C}$

In principle the following statement should never be executed.

write (Iun_output, 605)

goto 17

28

continue

C

$\mathrm{C}$

At this point we know the identity of the target, the region in which the interaction occurred, and the $z$ coordinate as measured along the z-axis from the end of the region that is closest to the origin. Now generate authentic $x$ and $z$ coordinates. The quantity delta_z that is returned by the code above is equal to delta_z only in the small angle approximation.

if (interaction_region.eq. 1) then

if (delta_z .le.1.16501e+03) then

$\mathrm{x}$ _coordinate_interaction $=0.0 \mathrm{e}$

$z_{-}$coordinate_interaction $=$delta_z

region_1_segment_1 = .true.

else

keep_this_angle = angle_zz_rl_sl

temp $=$ delta_z $-1.16501 e+03$

temp_z = temp / trig_factor_1

$x_{\text {_coordinate_interaction }}=$ slope_line_l 1 temp_z

$z_{-}$coordinate_interaction $=$temp_ $\bar{z}+1.16501 e+0 \overline{3}$

region_1_segment_2 = .true. endif

keep_this_angle = angle_zz_r1_s2

elseif (interaction_region .eq. 2 ) then

if (delta_z.le. $3.00 e+02$ ) then

temp_z = delta_z / trig_factor_1

temp_x = slope_line_1 * temp_z

$\$$

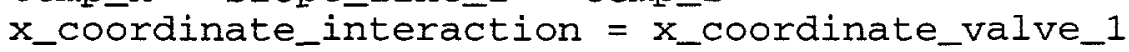

+ temp_x

z_coordinate_interaction $=z_{\text {_coordinate_valve_l }}$

$\$$

+ temp_z

region_2_segment_1 = .true. 
else

keep_this_angle $=$ angle_zz_r2_sl

delta_z = delta_z - 3.00e+02

temp_z $=$ delta_z / trig_factor_2

temp_x $=$ slope_line_2 * temp_z

$x$ _coordinate_interaction $=x$ _coord_do + temp_x

$z$ _coordinate_interaction $=z_{\text {_coord_do }}$

$\$$ endif

region_2_segment_2 = .true.

keep_this_angle = angle_zz_r2_s2

elseif (interaction_region .eq. 3) then

region_3 $=$.true.

keep_this_angle $=$ angle_zz_r3

temp_z $=$ delta_z/trig_factor_2

temp_x = slope_line_2 * temp_z

$\$$

$x \_c o o r d i n a t e \_$interaction $=x_{\text {_coordinate_valve_2 }}$

+ temp_x

z_coordinate_interaction $=z$ _coordinate_valve_2

$\$$

else

if (delta_z.le. length_r4_segment_1) then

region_4_segment_1 = .true.

keep_this_angle $=$ angle_zz_r4_s1

temp_z = delta_z/trig_factor_2

temp_x = slope_line_2 * temp_z

$\$$

$x$ _coordinate_interaction $=x$ _coordinate_valve_3

$\$$

else

z_coordinate_interaction $=z_{\text {_coordinate_valve_3 }}$

endif

delta_z = delta_z - length_r4_segment_1

if (delta_z.le. length_r4_segment_2) then

region_4_segment_2 = .true.

keep_this_angle = angle_zz_r4_s2

temp_z = delta_z/trig_factor_3

temp_x = slope_line_3 * temp_z

$\mathrm{x}$ _coordinate_interaction $=\mathrm{x}$ _coord_d5_end + temp_x else

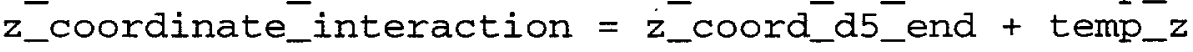
endif

delta_z = delta_z - length_r4_segment_2

if (delta_z.le. length_r4_segment_3) then

region_4_segment_3 = . true.

keep_this_angle $=$ angle_zz_r4_s3

temp_z = delta_z/trig_factor_4

temp_x $=$ slope_line_4 ${ }^{*}$ temp_z

$x \_c o o r d i n a t e \_$interaction $=x$ _coord_d6_end + temp_x

else

$z_{\text {_coordinate_interaction }}=z_{\text {_coord_d6_end }}+$ temp_z

region_4_segment_4 = .true.

keep_this_angle = angle_zz_r4_s4

delta_z = delta_z - length_r4_segment_3

temp_z $=$ delta_z/trig_factor_4

temp_ $x=$ slope_line_4 ${ }_{\bar{*}}$ temp_z

endif

$x$ _coordinate_interaction $=x$ _coord_d8_end + temp_x

endif

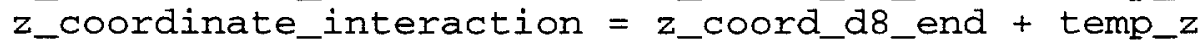


if (interaction_region .eq. 1) then

if (z_coordinate_interaction. . le. 1.16501e+03) then else

$x$ _coordinate_interaction $=0.0 e 0$

$\$$

$\mathrm{x}$ _coordinate_interaction $=$ intercept_line_ $1+$

endif

slope_line_1 * z_coordinate_interaction

elseif (interaction_region .eq. 2) then

if (z_coordinate_interaction.le.2.22998e+03) then

$x$ _coordinate_interaction $=$ intercept_line_ $1+$

$\$$ slope_line_1 * z_coordinate_interaction

else

$x_{\text {_coordinate_interaction }}=$ intercept_line_2 +

$\$$

else

endif slope_line_2 * $z$ _coordinate_interaction

$x_{\text {_coordinate_interaction }}=$ intercept_line_2 $2+$

$\$ \quad$ slope_line_2 * $z_{\text {_coordinate_interaction }}$

endif

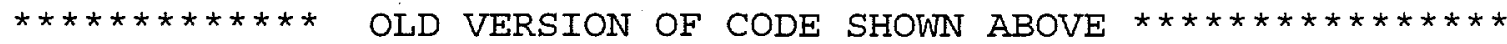

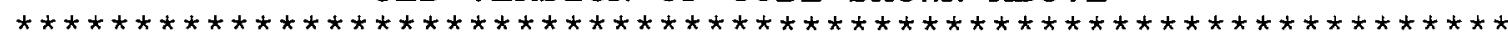

Note that the preceding code distributes the interactions over positive $z$ and negative $x$. Negative $x$ corresponds to the outside arc. These signs are appropriate for bombarding our muon arm with background but inappropriate if the objective is to examine particles

that pass through the second muon arm, the central volume of PHENIX, and enter our muon arm with approximately the same slope as particles that originate at the origin. Permit change of sign here.

$\mathrm{x} \_$coordinate_interaction $=$change_sign_ $\mathrm{x}$ *

$\$$ -

z_coordinate_interaction = change_sign_z *

\$ z_coordinate_interaction

Load the xyz "prebuffer" here.

$x y z(1)=x$ _coordinate_interaction

$\mathrm{xyz}(2)=0.0 \mathrm{e} 0$

xyz $(3)=z_{\text {_coordinate_interaction }}$

$\mathrm{C}$

Store the location of the interactions.

if (hydrogen_interaction) then $z_{\text {_in_meters }}=\operatorname{abs}\left(z_{\text {_coordinate_interaction }}\right) / 1.0 \mathrm{e}+02$

call serve(z_in_meters, lower_lim_z_plot, upper_lim_z_plot,

$\$$ bin_width_z_plot, index)

if (index .ge. 1 . and. index. le. no_bins_z_plot) then endif

$z$ plot_hydrogen (index) = z_plot_hydrogen(index) +1.000

elseif (helium_interaction) then

$z_{\text {_in_meters }}=$ abs $\left(z_{\text {_ccordinate_interaction }}\right) / 1.0 e+02$

$\$$

call serve (z_in_meters, lower_lim_z_plot, upper_lim_z_plot, bin_width_z_plot, index)

if (index .ge. 1 . and. index. le. no_bins_z_plot) then

z_plot_helium (index) $=$ z_plot_helium(index) $+1.0 e 0$

else endif

z_in_meters $=$ abs $\left(z_{-}\right.$coordinate_interaction $) / 1.0 e+02$

$\$ \quad$ call serve (z_in_meters, lower

if (index.ge. 1 . and. index. le. no_bins_z_plot) then 

endif endit

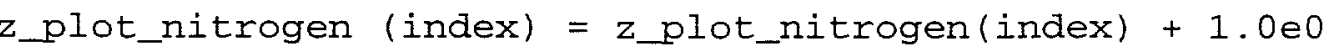

Read in the event number and the number of particles produced in the event.

if (hydrogen_interaction) then

lun_input $=$ lun_hydrogen

number_hydrogen_int $=$ number_hydrogen_int +1 goto $2 \overline{6}$

elseif (helium_interaction) then

lun_input = lun_helium

number_helium_int $=$ number_helium_int +1

else goto $2 \overline{6}$

endif

lun_input $=$ lun_nitrogen

number_nitrogen_int $=$ number_nitrogen_int +1 goto $2 \overline{6}$

continue

numevt $=$ numevt +1

read(Iun_input, 1002 , end $=17$ ) car1, ievent, mxtot_temp, car2, $\$$ btot, car3, phib

write (lun_output, 1003) ievent, mxtot_temp

mxtot $=0$

Begin loop over produced particles in this event.

DO 1 I=1, mxtot_temp

The following block of code was added by PNK on september 21 , 1995, in order to eliminate tracking of the so-called "spectator" nucleons.

read(lun_input, 1001) idtemp, 1bl, p2_temp, p3_temp,

$\$$ p4_temp, p1_temp if (idtemp .eq. 13 .or. idtemp .eq. 14) then go to 1

else

mxtot $=$ mxtot +1

We must specify the angle between the direction of motion and the positive $z$ axis of the PHENIX coordinate system. these angles are stored in the variables angle_zz_r4_sl, etc.

pptot $(2, \operatorname{mxtot})=\mathrm{p} 4$ _temp * sind (keep_this_angle)

$\$$

pptot $(4$, mxtot $)=p 4$ _temp $*$ cosd (keep_this_angle)

- p2_temp * sind (keep_this_angle)

pptot $(3, \mathrm{mxtot})=\mathrm{p} 3$-temp

pptot $(1, \mathrm{mxtot})=$ p1_temp

endif

idtot $($ mxtot $)=$ idtemp

$\mathrm{C}$

The following two lines were commented out by PNK on September 21, 1995 .

read(lun_input, 1001) idtot(I), 1bl, pptot(2,I), pptot(3, I), $\$ \quad \operatorname{pptot}(4, I), \operatorname{pptot}(1, I)$

These particles are their own parents.

id_parent $(i)=i d t o t(i)$

id parent (mxtot) = idtot (mxtot) 
Transfer subscripted variables to non-subscripted variables.

$\mathrm{px}=\operatorname{pptot}(2, I)$

py $=$ pptot $(3, I)$

$\mathrm{pz}=$ pptot $(4, I)$

igeant $=$ idtot $(I)$

$\mathrm{px}=$ pptot $(2, \mathrm{mxtot})$

py $=$ pptot $(3, m x t o t)$

$\mathrm{pz}=$ pptot $(4, \mathrm{mxtot})$

igeant $=$ idtot (mxtot)

total_momentum $=\operatorname{sqrt}(p x * p x+p y * p y+p z * p z)$

azimuthal_angle $=\operatorname{atan} 2 d(p y, p x)$

cosine_of_angle $=\mathrm{pz} /$ total_momentum

zenith_angle = acosd (cosine_of_angle)

$\mathrm{C}$

$\mathrm{C}$

C

$\mathrm{C}$

C

C

C

C

C

Count the number of particles of each type.

if (igeant.ge. 1 .and. igeant.le. 32) then i_cnt particles (igeant) =

$\$ \quad$ i_cnt_particles (igeant) +1

elseif (igeant.ge. 33 .and. igeant.le. 50) then i_cnt_particles (33) = i_cnt_particles $(33)+1$

elseif (igeant.ge. 51 and. igeant.le. 112) then else i_cnt_particles $(34)=$ i_cnt_particles $(34)+1$ endi $\bar{f}$

i_cnt_particles $(35)=i$ cnt_particles $(35)+1$

Leave some space for counting the number of particles.

Increment distributions for positive pions.

if ( igeant .eq. 8 ) then number positive_pions $=$ number_positive_pions +1

$\$$ call serve (zenith_angle, theta_low, theta_high, if (index .ge. 1 and. index .le. no_theta_bins) then no_pi_plus_theta (index) = no_pi_plus_theta (index) endif

call serve (azimuthal_angle, $-180.0 e 0,180.0 e 0,10.0 e 0$, index)

if (index ge. 1 and. index.le. 36) then no_pi_plus_phi (index) = no_pi_plus_phi (index) +1.000 endif

call serve (cosine_of_angle, $-1.0 e 0,+1.0 e 0,5.0 e-02$, index)

if (index.ge. I and. index.le. no_cos_theta_bins) then no_pi_plus_cos_theta (index) =

endif no_pi_plus_cos_theta (index) $+1.0 e 0$

call serve (total_momentum, lower_lim_ptot, upper_lim_ptot, bin_width_ptot, index)

if ( index .ge. 1 .and. index.le. no_bins_ptot) then endif no_pi_plus_ptot $($ index) = no_pi_plus_ptot (index) +1.000

temp $=$ abs $(p z)$

call serve (temp, lower_lim_pz, upper_lim_pz, bin_width_pz, index)

if ( index.ge. 1 and. index . Ie. no_bins_pz) then endif no_pi_plus_pz (index) = no_pi_plus_pz (index) $+1.0 e 0$

endif

go to 1 
Increment distributions for negative pions.

if ( igeant .eq. 9 ) then

number_negative_pions = number_negative_pions $+1.0 e 0$

call serve (zenith_angle, theta_low, theta_high,

$\$$

$\$$

$\$$

$\$$

$\$$

$\$$

$\$$

$\$$

endif

temp $=$ abs $(p z)$

call serve (temp, lower_lim_pz, upper_lim_pz,

endif theta_bin_width, index)

if (index.ge. 1 .and. index .le. no_theta_bins) then no_pi_minus_theta (index) = no_pi_minus_theta (index)

endif

call serve (azimuthal_angle, $-180.0 \mathrm{e}, 180.0 \mathrm{e} 0,10.0 \mathrm{e}$, index)

if (index.ge. 1 and. index.le. 36) then endif no_pi_minus_phi (index) = no_pi_minus_phi (index) +1.000

call serve (cosine_of_angle, $-1.0 e 0,+1.0 e 0,5.0 e-02$, index)

if (index.ge. 1 .and. index.1e. no_cos_theta_bins) then no_pi_minus_cos_theta (index) =

call serve (total_momentum, lower_lim_ptot, upper_lim_ptot, bin_width_ptot, index)

if ( index .ge. 1 .and. index .le. no_bins_ptot) then no_pi_minus_ptot (index) = no_pi_minus_ptot (index)

$$
+1.0 \mathrm{e} 0
$$

\section{bin_width_pz, index}

if ( index.ge. 1 and. index.le. no_bins_pz) then

endif no_pi_minus_pz $($ index $)=$ no_pi_minus_pz $($ index $)+1.0 e 0$

$\mathrm{C}$

Increment distributions for neutral pions.

if ( igeant.eq. 7 ) then

number_neutral_pions = number_neutral_pions $+1.0 e 0$

$\$$

call serve (zenith_angle, theta_low, theta_high,

if (index.ge. 1 . and. index.le. no_theta_bins) then no_pi_naught_theta (index) = no_pi_naught_theta (index)

$\$$

endif

call serve (azimuthal_angle, $-180.0 e 0,180.0 e 0,10.0 e 0$, index)

$\$$

if (index .ge. 1 and. index .le. 36) then no_pi_naught_phi (index) = no_pi_naught_phi (index)

endif

call serve (cosine_of_angle, $-1.0 e 0,+1.0 e 0,5.0 e-02$,

if (index.ge. 1 and. index.le. no_cos_theta_bins) then no_pi_naught_cos_theta (index) =

endif no_pi_naught_cos_theta (index) + 1.0e0

call serve (total_momentum, lower_lim_ptot, upper_lim_ptot, bin_width_ptot, index)

if (index.ge. 1 and. index.le. no_bins_ptot) then no_pi_naught_ptot (index) = no_pi_naught_ptot (index)

endif

$$
+1.0 e 0
$$

temp $=a b s(p z)$ 
call serve (temp, lower_lim_pz, upper_lim_pz,

bin_width_pz, index)

$\$$

if ( index.ge. 1 .and. index.le. no_bins_pz) then no_pi_naught_pz (index) = no_pi_naught_pz (index)

endif

endif

$+1.0 \mathrm{e} 0$

$\mathrm{C}$

Increment distributions for positive kaons.

if ( igeant.eq. 11 ) then

number_positive_kaons $=$ number_positive_kaons +1.000

$\$$

call serve (zenith_angle, theta_low, theta_high, theta_bin_width, index)

$\$$

if (index.ge. 1 and. index.le. no_theta_bins) then no_ka_plus_theta (index) = no_ka_plus_theta (index)

endif

call serve (azimuthal_angle, $-180.0 \mathrm{e} 0,180.0 \mathrm{e} 0,10.0 \mathrm{e}$, index)

if (index .ge. 1 and. index .le. 36) then endif no_ka_plus_phi (index) = no_ka_plus_phi (index) $+1.0 e 0$

call serve (cosine_of_angle, $-1.0 e 0,+1.0 e 0,5.0 e-02$,

if (index .ge. 1 and. index.le. no_cos_theta_bins) then no_ka_plus_cos_theta (index) =

endif

call serve (total_momentum, lower_lim_ptot, upper_lim_ptot,

bin_width_ptot, index)

if ( index.ge. 1 and. index.le. no_bins_ptot) then no_ka_plus_ptot (index) = no_ka_plus_ptot (index)

endif $+1.0 \bar{e} 0$

temp $=\operatorname{abs}(p z)$

call serve (temp, lower_lim_pz, upper_lim_pz,

bin_width_pz, index)

if ( index.ge. 1 and. index .le. no_bins_pz) then

endif no_ka_plus_pz $($ index $)=$ no_ka_plus_pz (index) $+1.0 e 0$

endif

go to 1

Increment distributions for negative kaons.

if ( igeant.eq. 12 ) then

number_negative_kaons = number_negative_kaons $+1.0 \mathrm{e}$

$\$$

call serve (zenith_angle, theta_low, theta_high, theta_bin_width, index)

if (index.ge. 1 and. index .le. no_theta_bins) then

$\$$ no_ka_minus_theta (index) = no_ka_minus_theta (index)

endif

call serve (azimuthal_angle, $-180.0 \mathrm{e}, 180.0 \mathrm{e}, 10.0 \mathrm{e}$,

index)

if (index.ge. 1 .and. index.le. 36) then endif no_ka_minus_phi $($ index) $=$ no_ka_minus_phi (index) +1.000

call serve (cosine_of_angle, $-1.0 e 0,+1.0 e 0,5.0 e-02$, index)

if (index.ge. 1 .and. index.le. no_cos_theta_bins) then no_ka_minus_cos_theta (index) = no_ka_minus_cos_theta (index) + 1.0e0

endif 
call serve (total_momentum, lower_lim_ptot, upper_lim_ptot, bin_width_ptot, index)

if ( index .ge. 1 and. index.le. no bins ptot) then no_ka_minus_ptot (index) = no_ka_minus_ptot (index)

endif

$$
+1.0 \mathrm{e} \overline{0}
$$

temp $=\operatorname{abs}(p z)$

call serve (temp, lower_lim_pz, upper_lim_pz,

$\$$ bin_width_pz, index)

if ( index.ge. 1 . and. index.le. no_bins_pz) then endif no_ka_minus_pz (index) = no_ka_minus_pz (index) $+1.0 e 0$

endif

go to 1

C

$\mathrm{C}$

endif

endif

endif

endif

Increment distributions for neutral kaons.

if ( igeant .eq. 10 .or. igeant .eq. 16 ) then number_neutral_kaons $=$ number_neutral_kaons $+1.0 e 0$

call serve (zenith_angle, theta_low, theta_high, theta_bin_width, index)

if (index.ge. 1 .and. index .le. no_theta_bins) then no_ka_naught_theta (index) = no_ka_naught_theta (index)

call serve (azimuthal_angle, $-180.0 e 0,180.0 e 0,10.0 e 0$, index)

if (index.ge. 1 and. index.1e. 36) then no_ka_naught_phi (index) = no_ka_naught_phi (index)

call serve (cosine_of_angle, $-1.0 e 0,+1.0 e 0,5.0 e-02$, index)

if (index.ge. 1 and. index.le. no_cos_theta_bins) then no_ka_naught_cos_theta (index) =

endif no_ka_naught_cos_theta(index) $+1.0 e 0$

call serve (total_momentum, lower_lim_ptot, upper_lim_ptot, bin_width_ptot, index)

if ( index .ge. 1 .and. index.le. no_bins_ptot) then no_ka_naught_ptot (index) = no_ka_naught_ptot (index)

endif

temp $=$ abs $(p z)$

call serve (temp, lower_lim_pz, upper_lim_pz, bin_width_pz, index)

if ( index.ge. 1 and. index.le. no_bins_pz) then no_ka_naught_pz (index) = no_ka_naught_pz (index) +1.000

go to 1

$\mathrm{C}$

C

\section{Increment distributions for protons.}

if ( igeant.eq. 14 ) then number_protons $=$ number protons $+1.0 e 0$

call serve (zenith_angle, theta_low, theta_high, theta_bin_width, index)

if (index.ge. 1 and. index.le. no_theta_bins) then no_protons_theta (index) = no_protons_theta (index)

endif $+1.0 \mathrm{e} 0$

call serve (azimuthal_angle, $-180.0 \mathrm{e} 0,180.0 \mathrm{e} 0,10.0 \mathrm{e}$, index)

if (index.ge. 1 .and. index .le. 36) then no_protons_phi (index) = no_protons_phi (index) $+1.0 e 0$ 
endif

call serve (cosine_of_angle, $-1.0 \mathrm{e} 0,+1.0 \mathrm{e}, 5.0 \mathrm{e}-02$,

\section{if (index .ge. 1 and. index.1e. no_cos_theta_bins) then} no_protons_cos_theta (index) =

endif no_protons_cos_theta (index) + $1.0 \mathrm{e} 0$

call serve (total_momentum, lower_lim_ptot, upper_lim_ptot, bin_width_ptot, index)

if ( index.ge. 1 . and. index .le. no_bins_ptot_nucleon) then endif no_protons_ptot $($ index) $=$ no_protons_ptot (index) $+1.0 e 0$

temp $=\operatorname{abs}(p z)$

call serve (temp, lower_lim_pz, upper_lim_pz,

bin_width_pz, index)

if ( index.ge. 1 and. index .le. no_bins_ptot_nucleon) then endif no_protons_pz (index) = no_protons_pz (index) +1.000

endif

go to 1

C

$$
\text { endif }
$$

endif

endif

Increment distributions for neutrons.

if ( igeant.eq. 13 ) then

number_neutrons $=$ number_neutrons +1.0 e0

call serve (zenith_angle, theta_low, theta_high, theta_bin_width, index)

if (index.ge. 1 and. index. Ie. no_theta_bins) then no_neutrons_theta (index) = no_neutrons_theta (index)

call serve (azimuthal_angle, $-180.0 \mathrm{e} 0,180.0 \mathrm{e} 0,10.0 \mathrm{e} 0$, index)

if (index.ge. 1 and. index.1e. 36) then endif no_neutrons_phi (index) = no_neutrons_phi (index) +1.0 e0

call serve (cosine_of_angle, $-1.0 e 0,+1.0 e 0,5.0 e-02$, if (index.ge. 1 and. index.1e. no_cos_theta_bins) then no_neutrons_cos_theta (index) =

endif no_neutrons_cos_theta (index) + $1.0 e 0$

call serve (total_momentum, lower_lim_ptot, upper_lim_ptot, bin_width_ptot, index)

if ( index .ge. 1 and. index .le. no_bins_ptot_nucleon) then no_neutrons_ptot (index) = no_neutrons_ptot (index)

temp $=\operatorname{abs}(p z)$

call serve (temp, lower_lim_pz, upper_lim_pz, bin_width_pz, index)

if ( index.ge. 1 . and. index .le. no_bins_ptot_nucleon) then endif no_neutrons_pz (index) = no_neutrons_pz (index) $+1.0 e 0$ go to 1

$\mathrm{C}$

Increment distributions for gammas.

if ( igeant .eq. 1 ) then number_gammas $=$ number_gammas $+1.0 e 0$

call serve (zenith_angle, theta_low, theta_high, endif if (index .ge. 1 .and. index . le. no_theta_bins) then
no_gammas_theta (index) $=$ no_gammas_theta (index) +1.000 
call serve (azimuthal_angle, $-180.0 \mathrm{e} 0,180.0 \mathrm{e} 0,10.0 \mathrm{e}$, index)

if (index .ge. 1 .and. index .1e. 36) then endif no_gammas_phi $($ index) $=$ no_gammas_phi (index) $+1.0 e 0$

call serve (cosine_of_angle, $-1.0 e 0,+1.0 e 0,5.0 e-02$, index)

if (index.ge. 1 .and. index.le. no_cos_theta_bins) then no_gammas_cos_theta (index) = no_gammas_cos_theta (index) +1.000

endif

call serve (total_momentum, lower_lim_ptot, upper_lim_ptot, bin_width_ptot, index)

if ( index .ge. 1 .and. index.le. no_bins_ptot) then no_gammas_ptot $($ index) $=$ no_gammas_ptot (index) $+1.0 e 0$ endif

temp $=$ abs $(p z)$

call serve (temp, lower_lim_pz, upper_lim_pz,

if ( index.ge. 1 . and. index .le. no_bins_pz) then endif no_gammas_pz (index) = no_gammas_pz (index) $+1.0 e 0$

endif go to 1

Increment distributions for positrons.

if ( igeant .eq. 2 ) then number positrons $=$ number positrons $+1.0 e 0$

call serve (zenith_angle, theta_low, theta_high,

if (index.ge. 1 . and. index.le. no_theta_bins) then no_positrons_theta (index) = no_positrons_theta (index)

endif

call serve (azimuthal_angle, -180.0e0, 180.0e0, 10.0e0,

if (index no_positrons_phi (index) = no_positrons_phi (index)

endif $+1.0 \mathrm{e} 0$

call serve (cosine_of_angle, $-1.0 e 0,+1.0 e 0,5.0 e-02$, if (index.ge. 1 .and. index.le. no_cos_theta_bins) then no_positrons_cos_theta (index) =

endif no_positrons_cos_theta(index) + $1.0 e 0$

call serve (total_momentum, lower_lim_ptot, upper_lim_ptot, bin_width_ptot, index)

if ( index .ge. 1 . and. index .le. no_bins_ptot) then no_positrons_ptot (index) = no_positrons_ptót (index)

endif $+1.0 \mathrm{e} 0$

temp $=\operatorname{abs}(p z)$

call serve (temp, lower_lim_pz, upper_lim_pz, bin_width_pz, index)

if ( index.ge. 1 . and. index .le. no_bins_pz) then no_positrons_pz (index) = no_positrons_pz (index) $+1.0 e 0$ endif endif go to 1

$\mathrm{C}$

Increment distributions for electrons.

if ( igeant .eq. 3 ) then number_electrons $=$ number_electrons $+1.0 e 0$ 
call serve (zenith_angle, theta_low, theta_high, theta_bin_width, index)

if (index.ge. 1 and. index.le. no_theta_bins) then no_electrons_theta (index) = no_electrons_theta (index)

\section{endif} $+1.0 \mathrm{e}$

call serve (azimuthal_angle, $-180.0 e 0,180.0 e 0,10.0 e 0$, if (index .ge. 1 and. index .le. 36) then no_electrons_phi (index) = no_electrons_phi (index)

endif

call serve (cosine_of_angle, $-1.0 e 0,+1.0 e 0,5.0 e-02$, if (index.ge. 1 .and. index.le. no_cos_theta_bins) then no_electrons_cos_theta (index) = no_electrons_cos_theta(index) $+1.0 e 0$

endif

call serve (total_momentum, lower_lim_ptot, upper_lim_ptot, if ( index.ge. 1 .and. index.le. no_bins_ptot) then no_electrons_ptot (index) = no_electrons_ptot (index)

endif $+1.0 \mathrm{e} 0$

temp $=\operatorname{abs}(p z)$

call serve (temp, lower_lim_pz, upper_lim_pz, bin_width_pz, index)

if ( index .ge. 1 . and. index . le. no_bins_pz) then endif no_electrons_pz (index) $=$ no_electrons_pz (index) $+1.0 e 0$ endif

1 continue

An entire event has now been processed. Before proceeding to the next event, increment the numbers distributions.

temp = float (number_positive_pions)

call serve (temp,0.0, 99.0, 1.0, index)

if (index .ge. 1 and. index.1e. no_bins_pions) then no_pi_plus (index) = no_pi_plus (index) $+1.0 e 0$

endif

temp = float (number_negative_pions)

call serve (temp,0.0, 99.0, 1.0, index)

if (index.ge. 1 and. index.le. no_bins_pions) then

endif

no_pi_minus (index) = no_pi_minus (index) +1.000

temp = float (number_neutral_pions)

call serve (temp,0.0, 99.0, 1.0, index)

if (index.ge. 1 and. index.le. no_bins_pions) then endif

no_pi_naught (index) = no_pi_naught (index) +1.0e0

temp01 = float (no_bins_kaons - 1)

temp = float (number_neutral_kaons)

call serve (temp,0.0, temp01, 1.0, index)

if (index.ge. 1 and. index.le. no_bins_kaons) then endif no_kaon_o (index) $=$ no_kaon_o (index) $+1.0 e 0$

temp = float (number_positive_kaons)

call serve (temp,0.0, temp01, 1.0, index)

if (index ge. 1 and. index. le. no_bins_kaons) then endif no_kaon_plus (index) = no_kaon_plus (index) $+1.0 e 0$

temp = float (number_negative_kaons)

call serve (temp,0.0, temp01, 1.0, index)

if (index.ge. 1 .and. index. le. no_bins_kaons) then no_kaon_minus (index) = no_kaon_minus (index) $+1.0 e 0$ 
endif

temp = float (number_protons)

call serve (temp, 55.0, 104.0, 1.0, index)

if (index.ge. 1 and. index.le. no_bins_nucleons) then

no_protons (index) $=$ no_protons (index) $+1.0 e 0$

endif

temp = float (number_neutrons)

call serve (temp, $10 \overline{5} .0,154.0,1.0$, index)

if (index.ge. 1 and. index. Ie. no_bins_nucleons) then

no_neutrons (index) $=$ no_neutrons $($ index) $+1.0 e 0$

endif

temp = float (number_gammas)

temp01 = float (no_bins_gamma - 1)

call serve (temp,0.0, temp01, 1.0, index)

if (index.ge. 1 and. index.le. no_bins_gamma) then

endif no_gamma (index) $=$ no_gamma (index) $+\overline{1} .0 e 0$

temp01 = float (no_bins_leptons - 1)

temp = float (number_electrons)

call serve (temp,0.0, temp01, 1.0, index)

if (index .ge. 1 .and. index .le. no_bins_leptons) then endif no_electron (index) $=$ no_electron $($ index $)+1.0$ eo

temp = float (number_positrons)

cal1 serve (temp,0.0, temp 01, 1.0, index)

if (index.ge. 1 and. index.le. no_bins_leptons) then endif no positron (index) = no_positron (index) $+1.0 e 0$

$\mathrm{C}$

Set ievstat $=-1$ to signal a successful event.

ievstat $=-1$

return

continue

C

Arrive here if end of file was encountered or all 100 events read in.

Set the value of ievstat to +2 in order to signal the end of file.

ievstat $=2$

write (Iun_output, 80) (i_cnt_particles $(j), j=1,35$ )

write (Iun_output, 607) hydrogen_file, helium_file, nitrogen_file, $\$$ true_distributions

write (Iun_output,608) enable_region

write (lun_output, 609) probability_hydrogen, probability_helium, \$ probability_nitrogen

write (lun_output, 610) rel_prob_hydrogen, rel_prob_helium,

$\$$ rel_prob_nitrogen

write (Iun_output, 611) total_hydrogen_thickness,

\$ total_helium_thickness, total_nitrogen_thickness

write (Iun_output, 612) total_length_hydrogen,

$\$$ total_length_helium, totāl_length_nitrogen

write (lun_output,606) number_hydrogen_int, number_helium_int,

$\$$ number_nitrogen_int

C

Close the open hijet files.

if (hydrogen_file) then close (unit=lun_hydrogen, status='keep')

elseif (helium_file) then close (unit=lun_helium, status='keep')

elseif (nitrogen file) then close (unit= Iun_nitrogen, status $=$ 'keep') 
endif

Because of concern over too many open logical units, re-use the logical unit number for hydrogen since that file has been closed.

Write out the distributions in $z$ for each of the arrays z_plot_hydrogen, z_plot_helium, and z_plot_nitrogen.

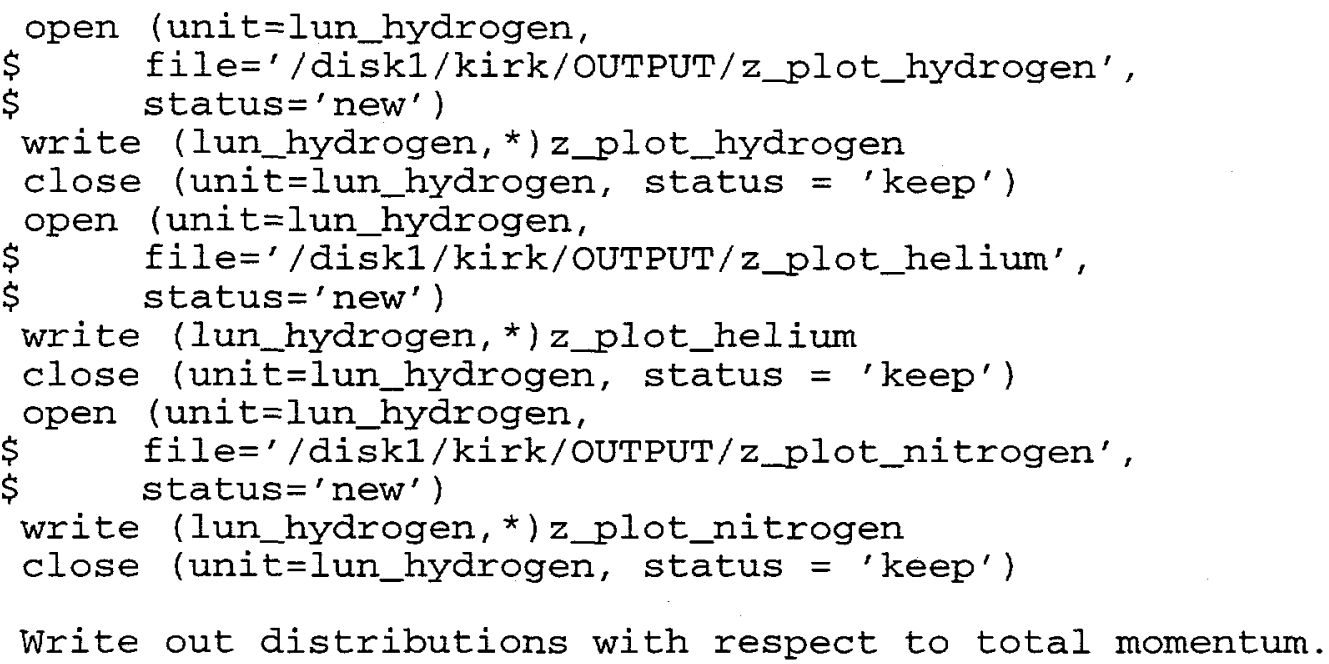

do $72 i=1$, no_bins_ptot

no_pi_plus_ptot $(i)=$ no_pi_plus_ptot $(i) /$ bin_width_ptot no_pi_minus_ptot $(i)=$ no_pi_minus_ptot (i) / bin_width_ptot no_pi_naught_ptot $(i)=$ no_pi_naught_ptot (i)/ bin_width_ptot no_ka_plus_ptot $(i)=$ no_ka_plus_ptot $(i) /$ bin_width_ptot no_ka_minus_ptot $(i)=$ no_ka_minus_ptot (i)/ bin_width_ptot no_ka_naught_ptot $(i)=$ no_ka_naught_ptot (i) / bin_width_ptot no_protons_ptot $(i)=$ no_protons_ptot $(i) /$ bin_width_ptot no_neutrons_ptot $(i)=$ no_neutrons_ptot $(i) /$ bin_width_ptot no_gammas_ptot $(i)=$ no_gammas_ptot (i) / bin_width_ptot no_electrons_ptot $(i)=$ no_electrons_ptot (i) $/$ bin_width_ptot no_positrons_ptot $(i)=$ no_positrons_ptot (i)/ bin_width_ptot 
file='/disk1/kirk/OUTPUT/ordinate_dka_naught_dptot', status $=$ 'new')

write (lun_hydrogen, *) no_ka_naught_ptot

close (unit=lun_hydrogen, status='keep')

open (unit=lun_hydrogen,

$\$ \quad$ file='/diskl/kirk/OUTPUT/ordinate_dproton_dptot' ,

$\$$ status='new')

write (Iun_hydrogen, *) no_protons_ptot

close (unit=lun_hydrogen, status='keep')

open (unit=lun_hydrogen,

$\$$ file='/disk1/kirk/oUTPUT/ordinate_dneutron_dptot',

$\$ \quad$ status $=$ 'new')

write (Iun_hydrogen, *) no_neutrons_ptot

close (unit=lun_hydrogen, status='keep')

open (unit=lun_hydrogen,

\$ file='/disk1/kirk/OUTPUT/ordinate_dgamma_dptot',

$\$$ status='new')

write (Iun_hydrogen, *) no_gammas_ptot

close (unit=lun_hydrogen, status='keep')

open (unit=lun_hydrogen,

$\$ \quad$ file='/disk1/kirk/OUTPUT/ordinate_delectron_dptot',

$\$$ status $=$ 'new')

write (lun_hydrogen, *) no_electrons_ptot

close (unit=lun_hydrogen, status='keep')

open (unit=lun_hydrogen,

\$ file='/disk1/kirk/OUTPUT/ordinate_dpositron_dptot',

$\$ \quad$ status $=$ 'new')

write (lun_hydrogen, *) no_positrons_ptot

close (unit=lun_hydrogen, status='keep')

$\mathrm{C}$

C

C

C

$\mathrm{C}$

201

write out distributions with respect to $\mathrm{pz}$.

do $201 i=1,150$

no_pi_plus_pz (i) = no_pi_plus_pz (i) / bin_width_pz

no_pi_minus_pz (i) = no_pi_minus_pz (i) / bin_width_pz

no_pi_naught_pz (i) = no_pi_naught_pz (i)/ bin_width_pz

no_ka_plus_pz (i) = no_ka_plus_pz (i)/ bin_width_pz

no_ka_minus_pz (i) = no_ka_minus_pz (i) / bin_width_pz

no_ka_naught_pz (i) = no_ka_naught_pz (i)/bin_width_pz

no_protons_pz $(\bar{i})=$ no_protons_pz (i) $/$ bin_width_pz

no_neutrons_pz (i) = no_neutrons_pz (i) $/$ bin_width_pz

no_gammas_pz $(i)=$ no_gammas_pz (i) / bin_width_pz

no_electrons_pz (i) = no_electrons_pz (i)/ bin_width_pz

no_positrons_pz (i) = no_positrons_pz (i)/ bin_width_pz

continue

open (unit=lun_hydrogen,

$\$ \quad \mathrm{file}=$ '/disk1/kirk/OUTPUT/ordinate_dpi_plus_dpz',

$\$ \quad$ status $=$ 'new')

write (lun_hydrogen, *) no_pi_plus_pz

close (unit=lun_hydrogen, status='keep')

open (unit=lun_hydrogen,

\$ file='/disk1/kirk/OUTPUT/ordinate_dpi_minus_dpz',

$\$$ status $=$ ' new')

write (lun_hydrogen, *) no_pi_minus_pz

close (unit=lun_hydrogen, status='keep')

open (unit=lun_hydrogen,

\$ file='/diski/kirk/OUTPUT/ordinate_dpi_naught_dpz',

$\$$ status $=$ 'new')

write (lun_hydrogen, *) no_pi_naught_pz

close (unit=lun_hydrogen, status='keep')

open (unit=lun_hydrogen,

\$ file='/diskl/kirk/OUTPUT/ordinate_dka_plus_dpz',

$\$$ status $=$ 'new')

write (Iun_hydrogen, *) no_ka_plus_pz

close (unit=lun_hydrogen, status='keep')

open (unit=lun_hydrogen, 
\$ file='/disk1/kirk/oUTPUT/ordinate_dka_minus_dpz',

$\$$ status='new')

write (lun_hydrogen, *) no_ka_minus_pz

close (unit=lun_hydrogen, status='keep')

open (unit=lun_hydrogen,

$\$ \quad \mathrm{file}=$ '/disk1/kirk/OUTPUT/ordinate_dka_naught_dpz',

$\$$ status $=$ 'new')

write (Iun_hydrogen, *) no_ka_naught_pz

close (unit=lun_hydrogen, status='keep')

open (unit=lun_hydrogen,

$\$$ file='/diskl/kirk/OUTPUT/ordinate_dproton_dpz',

$\$$ status='new')

write (lun hydrogen, *) no protons pz

close (unit=lun_hydrogen, status='keep')

open (unit=lun_hydrogen,

$\$ \quad \mathrm{file}=$ '/disk1/kirk/OUTPUT/ordinate_dneutron_dpz',

$\$$ status='new')

write (lun_hydrogen, *) no_neutrons_pz

close (unit=lun_hydrogen, status='keep')

open (unit=lun_hydrogen,

\$ file='/diski/kirk/OUTPUT/ordinate_dgamma_dpz',

$\$$ status $=$ 'new')

write (lun_hydrogen, *) no_gammas_pz

close (unit=lun_hydrogen, status='keep')

open (unit=lun_hydrogen,

\$ file='/disk1/kirk/OUTPUT/ordinate_delectron_dpz',

$\$$ status $=$ 'new')

write (Iun_hydrogen, *) no_electrons_pz

close (unit=Iun_hydrogen, status='keep')

open (unit=lun_hydrogen,

\$ file='/diskl/kirk/OUTPUT/ordinate_dpositron_dpz',

$\$$ status='new')

write (Iun_hydrogen, *) no_positrons_pz

close (unit=lun_hydrogen, status='keep')

Write out distributions with repect to azimuthal angle.

do $202 i=1,36$

no_pi_plus_phi (i) = no_pi_plus_phi (i) $/ 1.0 e+01$

no_pi_minus_phi $(i)=$ no_pi_minus_phi (i) $/ 1.0 e+01$

no_pi_naught_phi $(i)=$ no_pi_naught_phi (i) $/ 1.0 e+01$

no_ka_plus_phi (i) = no_ka_plus_phi (i) $/ 1.0 e+01$

no_ka_minus_phi $(i)=$ no_ka_minus_phi (i) $/ 1.0 e+01$

no_ka_naught_phi $(i)=$ no_ka_naught_phi (i) $/ 1.0 e+01$

no_protons_phi $(i)=$ no_protons_phi (i) $/ 1.0 e+01$

no_neutrons_phi (i) $=$ no_neutrons_phi (i) $/ 1.0 e+01$

no_gammas_phi $(i)=$ no_gammas_phi (i) $/ 1.0 e+01$

no_electrons_phi $(i)=$ no_electrons_phi (i) $/ 1.0 e+01$

no_positrons_phi $(i)=$ no_positrons_phi (i) $/ 1.0 e+01$

202 continue

open (unit=lun_hydrogen,

$\$$ file='/diski/kirk/OUTPUT/ordinate_dpi_plus_dphi',

$\$$ status $=$ 'new')

write (lun_hydrogen, *) no_pi_plus_phi

close (unit=lun_hydrogen, status='keep')

open (unit=lun_hydrogen,

\$ file='/diski/kirk/OUTPUT/ordinate_dpi_minus_dphi',

$\$$ status $=$ 'new')

write (lun_hydrogen, *) no_pi_minus_phi

close (unit=lun_hydrogen, status='keep')

open (unit=lun_hydrogen,

$\$$ file='/disk1/kirk/ouTPuT/ordinate_dpi_naught_dphi',

$\$$ status='new')

write (Iun_hydrogen, *) no_pi_naught_phi

close (unit=lun_hydrogen, status='keep')

open (unit=lun_hydrogen, 
file='/disk1/kirk/OUTPUT/ordinate_dka_plus_dphi',

status ='new')

write (lun_hydrogen, *) no_ka_plus_phi

close (unit=lun_hydrogen, status='keep')

open (unit=lun_hydrogen,

\$ file='/disk1/kirk/OUTPUT/ordinate_dka_minus_dphi',

$\$$ status $=$ 'new')

write (lun_hydrogen, *) no_ka_minus_phi

close (unit=lun_hydrogen, status='keep')

open (unit=lun_hydrogen,

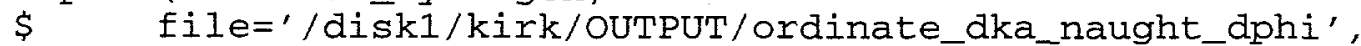

$\$$ status='new')

write (lun_hydrogen, *) no_ka_naught_phi

close (unit=lun_hydrogen, status='keep')

open (unit=lun_hydrogen,

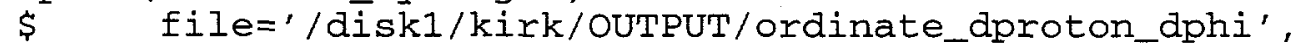

$\$$ status $=$ 'new')

write (lun_hydrogen, *) no_protons_phi

close (unit=lun_hydrogen, status='keep')

open (unit=lun_hydrogen,

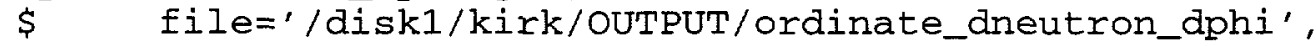

$\$$ status $=$ 'new')

write (lun_hydrogen, *) no_neutrons_phi

close (unit=lun_hydrogen, status='keep')

open (unit=lun_hydrogen,

$\$$

file='/disk1/kirk/OUTPUT/ordinate_dgamma_dphi',

status =' new')

write (Iun_hydrogen, *) no_gammas_phi

close (unit=lun_hydrogen, status='keep')

open (unit=lun_hydrogen,

\$ file='/disk1/kirk/OUTPUT/ordinate_delectron_dphi',

$\$ \quad$ status $=$ 'new')

write (lun_hydrogen, *) no_electrons_phi

close (unit=lun_hydrogen, status='keep')

open (unit=lun_hydrogen,

$\$ \quad \mathrm{file}=$ '/diskl/kirk/OUTPUT/ordinate_dpositron_dphi',

$\$$ status $=$ 'new')

write (lun hydrogen, *) no positrons_phi

close (unit=lun_hydrogen, status='keep')

Write out distributions with respect to zenith angle.

do $203 i=1,36$

no_pi_plus_theta $(i)=$ no_pi_plus_theta $(i) / 5.0 e 0$

no_pi_minus_theta $(i)=$ no_pi_minus_theta $(i) / 5.0 e 0$

no_pi_naught_theta $(i)=$ no_pi_naught_theta (i) $/ 5.000$

no_ka_plus_theta $(i)=$ no_ka_plus_theta (i) $/ 5.0 e 0$

no_ka_minus_theta $(i)=$ no_ka_minus_theta (i) $/ 5.000$

no_ka_naught_theta $(i)=$ no_ka_naught_theta (i) $/ 5.0 e 0$

$\mathrm{C}$ no_protons_theta $(i)=$ no_protons_theta $(i) / 5.0$ e0

no_neutrons_theta $(i)=$ no_neutrons_theta $(i) / 5.0 e 0$

no_gammas_theta $(i)=$ no_gammas_theta (i) $/ 5.0 e 0$

no_electrons_theta $(i)=$ no_electrons_theta (i) $/ 5.0 e 0$

no_positrons_theta $(i)=$ no_positrons_theta $(i) / 5.000$

203 continue

open (unit=lun_hydrogen,

\$ file='/diski/kirk/OUTPUT/ordinate_dpi_plus_dtheta',

$\$ \quad$ status $=$ 'new')

write (lun_hydrogen, *) no_pi_plus_theta

close (unit=lun_hydrogen, status='keep')

open (unit=lun_hydrogen,

\$ file='/disk1/kirk/OUTPUT/ordinate_dpi_minus_dtheta',

$\$$ status $=$ 'new')

write (lun_hydrogen, *) no_pi_minus_theta

close (unit=lun_hydrogen, status='keep')

open (unit=lun_hydrogen, 
write (lun_hydrogen, *) no_pi_naught_theta

close (unit=lun_hydrogen, status='keep')

open (unit=lun_hydrogen,

\$ $\quad$ ile='/disk1/kirk/ouTPuT/ordinate_dka_plus_dtheta',

$\$$ status $=$ 'new')

write (lun_hydrogen, *) no_ka_plus_theta

close (unit=lun_hydrogen, status='keep')

open (unit=lun_hydrogen,

\$ file='/disk1/kirk/OUTPUT/ordinate_dka_minus_dtheta',

$\$$ status $=$ 'new')

write (lun_hydrogen, *) no_ka_minus_theta

close (unit=lun_hydrogen, status=' keep')

open (unit=lun_hydrogen,

\$ file='/disk1/kirk/OUTPUT/ordinate_dka_naught_dtheta',

$\$$ status='new')

write (lun_hydrogen, *) no_ka_naught_theta

close (unit=lun_hydrogen, status='keep')

open (unit=lun_hydrogen,

$\$ \quad \mathrm{file}=$ '/disk1/kirk/OUTPUT/ordinate_dproton_dtheta',

$\$$ status='new')

write (lun_hydrogen, *) no_protons_theta

close (unit=lun_hydrogen, status='keep')

open (unit=lun_hydrogen,

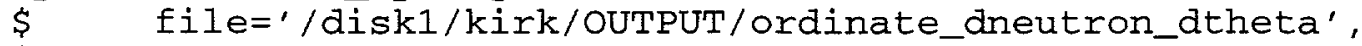

$\$$ status='new')

write (Iun_hydrogen, *) no_neutrons_theta

close (unit=lun_hydrogen, status='keep')

open (unit=lun_hydrogen,

\$ file='/disk1/kirk/OUTPUT/ordinate_dgamma_dtheta',

$\$$ status $=$ 'new')

write (lun_hydrogen, *) no_gammas_theta

close (unit=lun_hydrogen, status='keep')

open (unit=lun_hydrogen,

\$ file='/diskl/kirk/OUTPUT/ordinate_delectron_dtheta',

$\$$ status='new')

write (Iun_hydrogen, *) no_electrons_theta

close (unit=lun_hydrogen, status='keep')

open (unit=lun_hydrogen,

$\$ \quad$ file='/disk1/kirk/OUTPUT/ordinate_dpositron_dtheta',

$\$$ status='new')

write (Iun_hydrogen, *) no_positrons_theta

C

Write out distributions with respect to cosine theta.

do $204 i=1$, no_cos_theta_bins

no_pi_plus_cos_theta (i) = no_pi_plus_cos_theta (i) $/ 5.0 e-02$

no_pi_minus_cos_theta $(i)=$ no_pi_minus_cos_theta $(i) / 5.0 e-02$

no_pi_naught_cos_theta $(i)=$ no_pi_naught_cos_theta (i)

$\$$

/ $5.0 e-\overline{0} 2$

no_ka_plus_cos_theta (i) = no_ka_plus_cos_theta (i) $/ 5.0 e-02$

no_ka_minus_cos_theta $(i)=$ no_ka_minus_cos_theta (i) $/ 5.0 e-02$

no_ka_naught_cos_theta $(i)=$ no_ka_naught_cos_theta $(i)$

$\$$

C

no_protons_cos_theta $(i)=$ no_protons_cos_theta (i) $/ 5.0 e-02$

no_neutrons_cos_theta $(i)=$ no_neutrons_cos_theta (i) $/ 5.0 e-02$ no_gammas_cos_theta (i) = no_gammas_cos_theta (i) $/ 5.0 \mathrm{e}-02$

no_electrons_cos_theta $(i)=$ no_electrons_cos_theta (i)

$\$$ / $5.0 e-02$

no_positrons_cos_theta $(i)=$ no_positrons_cos_theta (i)

$\$$ / $5.0 e-\overline{0} 2$

continue

open lunit=lun_hydrogen,

$\$ \quad$ file='/diskl/kirk/OUTPUT/ordinate_dpi_plus_dcos_theta',

$\$$ status $=$ 'new') 
write (lun_hydrogen, *) no_pi_plus_cos_theta

close (unit=lun_hydrogen, status='keep')

open (unit=lun_hydrogen,

$\$$

$\$$ status $=$ 'new')

write (lun_hydrogen, *) no_pi_minus_cos_theta

close (unit=lun_hydrogen, status='ḱkeep')

open (unit=lun_hydrogen,

$\$$

$\$$, status='new')

write (lun_hydrogen, *) no_pi_naught_cos_theta

close (unit=lun_hydrogen, status='keep')

open (unit=lun_hydrogen,

$\$$

$\$$ status $=$ 'new')

write (lun_hydrogen, *) no_ka_plus_cos_theta

close (unit=lun_hydrogen, status='keep')

open (unit=lun_hydrogen,

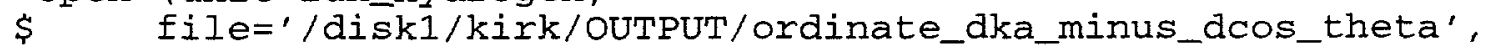

$\$$ status $=$ 'new')

write (lun_hydrogen, *) no_ka_minus_cos_theta

close (unit=lun_hydrogen, status=' keep')

open (unit=lun_hydrogen,

$\$$ file='/diskl/kirk/OUTPUT/ordinate_dka_naught_dcos_theta'

$\$$, status='new')

write (lun_hydrogen, *) no_ka_naught_cos_theta

close (unit $=$ lun_hydrogen, status='keep')

open (unit=lun_hydrogen,

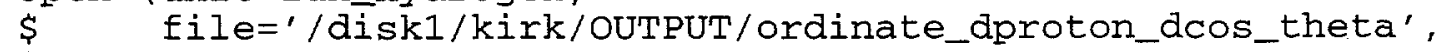

$\$$ status='new')

write (Iun_hydrogen, *) no_protons_cos_theta

close (unit $=1$ un_hydrogen, status='keep')

open (unit=lun hydrogen,

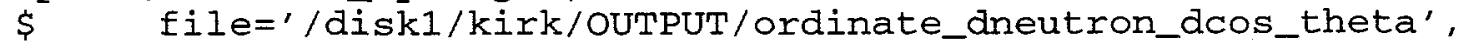

$\$$ status $=$ 'new')

write (lun_hydrogen, *) no_neutrons_cos_theta

close (unit=lun_hydrogen, status='keep')

open (unit=lun_hydrogen,

$\$$

$\$ \quad$ status $=$ 'new')

write (lun_hydrogen, *) no_gammas_cos_theta

close (unit=lun hydrogen, status='keep')

open (unit=lun_hydrogen,

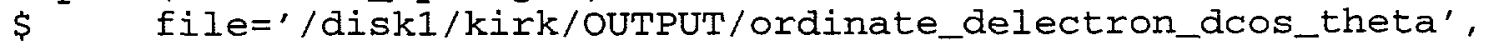

$\$$ status $=$ 'new')

write (lun_hydrogen, *) no_electrons_cos_theta

close (unit=Iun_hydrogen, status='keep')

open (unit=lun_hydrogen,

$\$ \quad f i l e=' / \mathrm{disk1} / \mathrm{kirk} /$ OUTPUT/ordinate_dpositron_dcos_theta',

$\$$ status $=$ 'new')

write (Iun_hydrogen, *) no_positrons_cos_theta

close (unit=lun_hydrogen, status='keep')

Now write out the numbers distributions

open (unit=lun_hydrogen,

\$ file='/diskl/kirk/OUTPUT/number_positive_pions',

$\$$ status $=$ 'new')

write (lun_hydrogen, *) no_pi_plus

close (unit=lun hydrogen, status='keep')

open (unit=lun_hydrogen,

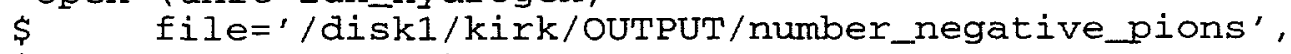

$\$$ status='new')

write (lun_hydrogen, *) no_pi_minus

close (unit=lun hydrogen, status='keep')

open (unit=lun_hydrogen, 
\$ file='/diskl/kirk/OUTPUT/number_neutral_pions',

$\$$ status $=$ 'new' $)$

write (lun_hydrogen, *) no_pi_naught

close (unit=lun_hydrogen, status='keep')

open (unit=lun_hydrogen,

$\$ \quad$ file='/disk1/kirk/OUTPUT/number_positive_kaons',

$\$$ status $=$ 'new')

write (lun_hydrogen, *) no_kaon_plus

close (unit=lun_hydrogen, status='keep')

open (unit=lun_hydrogen,

$\$$ file='/disk1/kirk/OUTPUT/number_negative_kaons',

$\$$ status $=$ 'new')

write (lun_hydrogen, *) no_kaon_minus

close (unit=lun_hydrogen, status='keep')

open (unit=lun hydrogen,

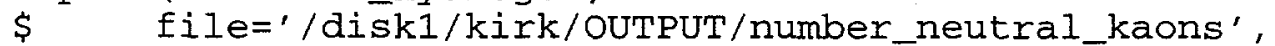

$\$$ status $=$ 'new')

write (lun_hydrogen, *) no_kaon_0

close (unit=lun_hydrogen, status='keep')

open (unit=lun_hydrogen,

$\$ \quad$ file='/disk1/kirk/OUTPUT/number_protons',

$\$$ status='new')

write (Iun_hydrogen, *) no_protons

close (unit=lun_hydrogen, status='keep')

open (unit=lun_hydrogen,

$\$ \quad \mathrm{file}=$ '/diskl/kirk/OUTPUT/number_neutrons',

$\$$ status $=$ 'new')

write (lun_hydrogen, *) no_neutrons

close (unit=lun_hydrogen, status='keep')

open (unit=lun_hydrogen,

$\$$ file='/disk1/kirk/OUTPUT/number_gammas',

$\$$ status $=$ 'new')

write (lun_hydrogen, *) no_gamma

close (unit=lun_hydrogen, status='keep')

open (unit=lun_hydrogen,

\$ file='/disk1/kirk/OUTPUT/number_electrons',

$\$$ status $=$ 'new')

write (lun_hydrogen, *) no_electron

close (unit=Iun_hydrogen, status='keep')

open (unit=lun_hydrogen,

$\$ \quad f i l e=' / d i s k 1 / k i r k / O U T P U T / n u m b e r \_p o s i t r o n s '$,

$\$$ status $=$ 'new')

write (lun_hydrogen, *) no_positron

close (unit=lun_hydrogen, status='keep')

close (unit=lun_output, status='keep')

return

.80 format (' ', 'NUMBER OF PARTICLES BY GEANT IDENTIFICATION NUMBER'

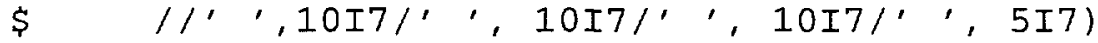

600 Eormat (' ', 'Execution ceased because no file was opened.')

601 format (',', 'Execution ceased because no region of the beamline $\$$ was activated.')

602 format ('',' 'Execution ceased because helium file was opened, tr $\$$ ue distributions were specified, but neither region of cold

$\$$ bore was enabled.')

603 format (',', 'Execution ceased because nitrogen file was opened, $t$ \$ rue distributions were specified, but neither region of warm $\$$ bore was enabled.')

604 format $('$, ,' Execution ceased because the program could not ident $\$$ ify the target.')

605 format (',', 'Execution ceased because of problem in determining $t$ $\$$ he $z$ coordinate of the interaction.')

606 format (' ',' Number of interactions with hydrogen $=$ ', I6, $/$,

$\$$ ' ', 'Number of interactions with helium $=$ ', I6,/,

$\$$ ' ', 'Number of interactions with nitrogen $=$ ', I6//)

607 format ('0','hydrogen file $=$ ', $114,2 \mathrm{x}$, 'helium file $=$ ', 114,

$\$ 2 x$, 'nitrogen file $=$ ', 114, $2 x$, 'true_distributions $=$ ', 114 
$\$ \quad / / 1$

608 format ('O', 'enable regions $=$ ', 414)

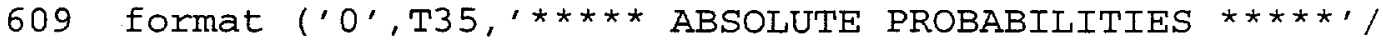

$\$$ ' ', 'hydrogen $=$ ', 1e12.5, 2x, 'helium $=$ ', 1e12.5, 2x, $\$$ 'nitrogen $=$ ', 1e12.5)

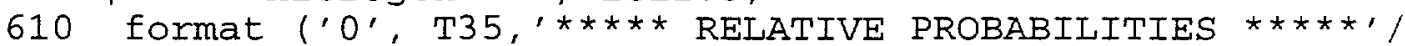

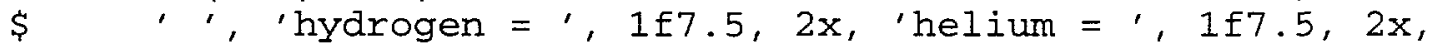

$\$$ 'nitrogen $=$ ', 1£7.5)

611 format ('O', 'total thickness of hydrogen $=$ ', 1 'e12.5, $2 \mathrm{x}$,

$\$$ 'per $\mathrm{cm}^{\star *} 2^{\prime} /{ }^{\prime}$ ', ' total thickness of helium $=1,1 \mathrm{e} 12.5,2 \mathrm{x}$,

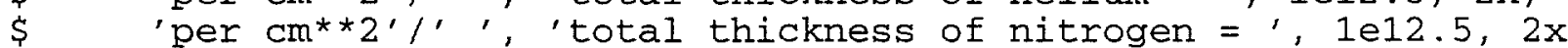

$\$ \quad, ' \operatorname{per} \mathrm{cm} * * 2 \prime)$

612 format ('O', 'total length of hydrogen $=$ ', 1e12.5, 2x,

$\$ \quad \mathrm{~cm}^{\prime} / \mathrm{\prime}^{\prime}$, ,' 'total length of helium $=$ ', 1e12.5, 2x,

$\$ \quad \mathrm{~cm}^{\prime} / \mathrm{\prime}^{\prime}$, ,' 'total length of nitrogen $=\prime, 1 e 12.5,2 \mathrm{x}$,

$\$$ ' $\mathrm{Cm}$ ')

1001 format(I9, a, 4F10.5)

1002 format (a, I4, I5, a, F10.5, a, F10.5)

1003 format (', 'Event number', $2 x$, i5, $2 x$, 'containing ', $2 x$, i5, $\$ 2 \mathrm{x}$, ' was readin from the hijet file.')

1101 format (a)

1102 format (', ', a)

end 


\author{
Appendix C \\ copies of transparencies shown \\ at
}

workshop on beam-gas interaction

BWL, July, I995 
Beam-Gas Interactions and Their Effect on the Level 1 Trigger of PHENIX

\author{
P. N. Kirk \\ Louisiana State University \\ and \\ C. Maguire \\ A. Trivedi \\ A. Rose \\ Vanderbilt University
}


Agenda

- I Ingredients of Simulation RHIC geometry

Cross Sections

Vacuum

Model for Interaction

- II Baric Numerology \& Event Rates

- III Characteristics of Reaction Products Number Distributions Momentum Distributions Angular Distributions

III Effects on Muon Arm

charged particle multiplicity level-1 trigger rates 
I work Schedule

complete analysis of PISA file charged particle multiplicity neutron multiplicity level -1 trigger rates study of interactions at $-z$ 


\section{Simulations Effort at LSU}

objective:

To Calculate the level 1 trigger rate due to beam-gas scattering

output:

Number for the triggering rate

Ascii file containing profile of events in $x-y$ plane at $z=0$

concluding report

tools:

PISA/PISORP 2.04

CERNLIB 94a

a little help from my friends

\section{Basic Numerology}

Luminosity (Au-Au)

Number $A u$ ions per bunch

time between bunches

pressure in warm bore

pressure in cold bore

beam gas in warm bore

beam gas in cold bore

approximate cross section for interaction

distance from $z=0$ to first bend
$2 \times 10^{26} \mathrm{~cm}^{-2} \mathrm{~s}^{-1}$

$1 \times 10^{9}$

$224 \times 10^{-9} s$

$5 \times 10^{-10}$ Torr

$<0.1 \times 10^{-10}$ Torr

$90 \% \mathrm{H}_{2}, 5 \% \mathrm{CO}, 5 \% \mathrm{CH}_{4}$

$95 \% \mathrm{H}_{2}, 5 \% \mathrm{He}$

$5 \times 10^{-24} \mathrm{~cm}^{2}$

approximately $100 \mathrm{~m}$

A simple calculation yields:

rate $=3.8 \mathrm{khz}$ 
- Flow of Analysis

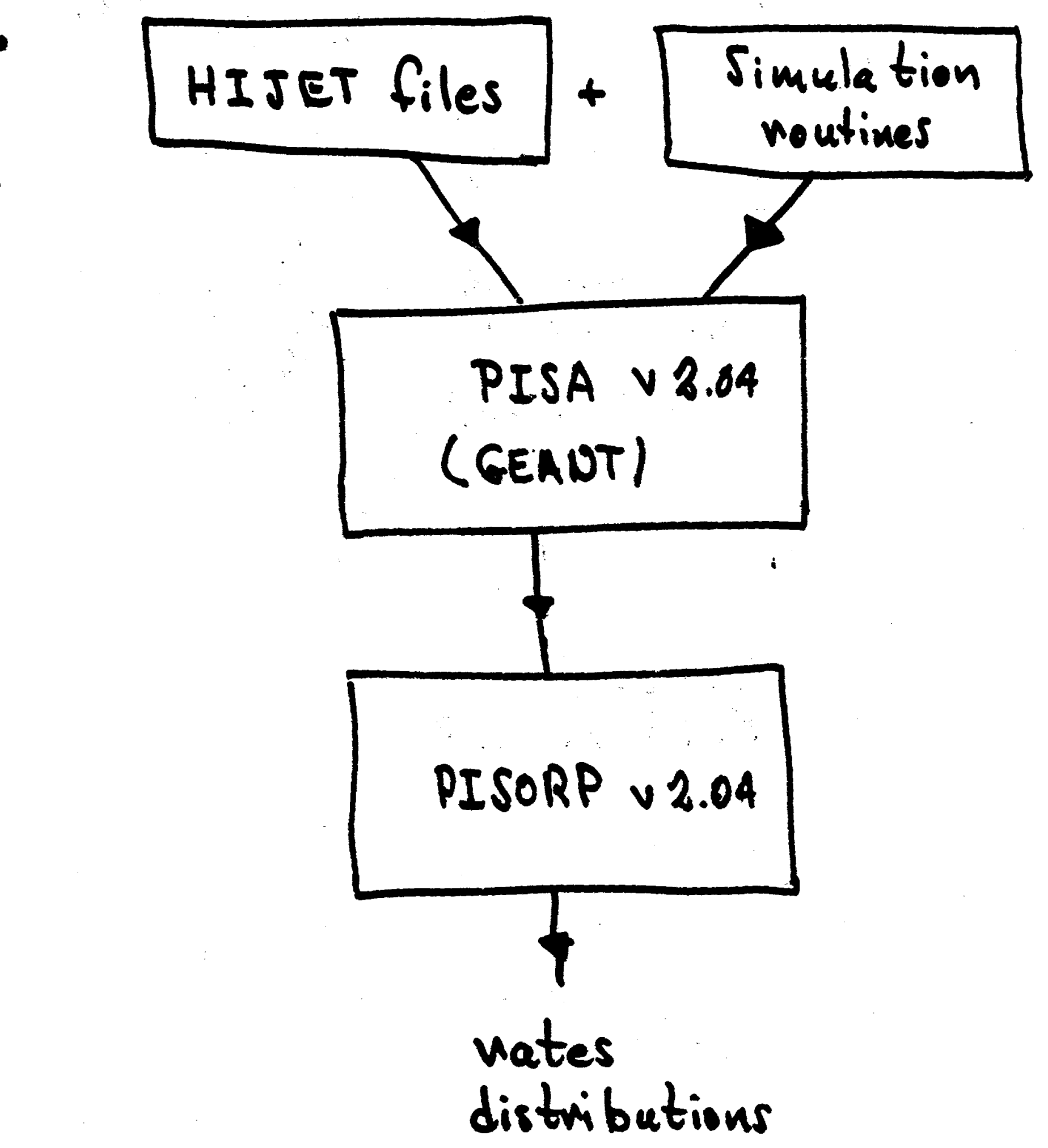




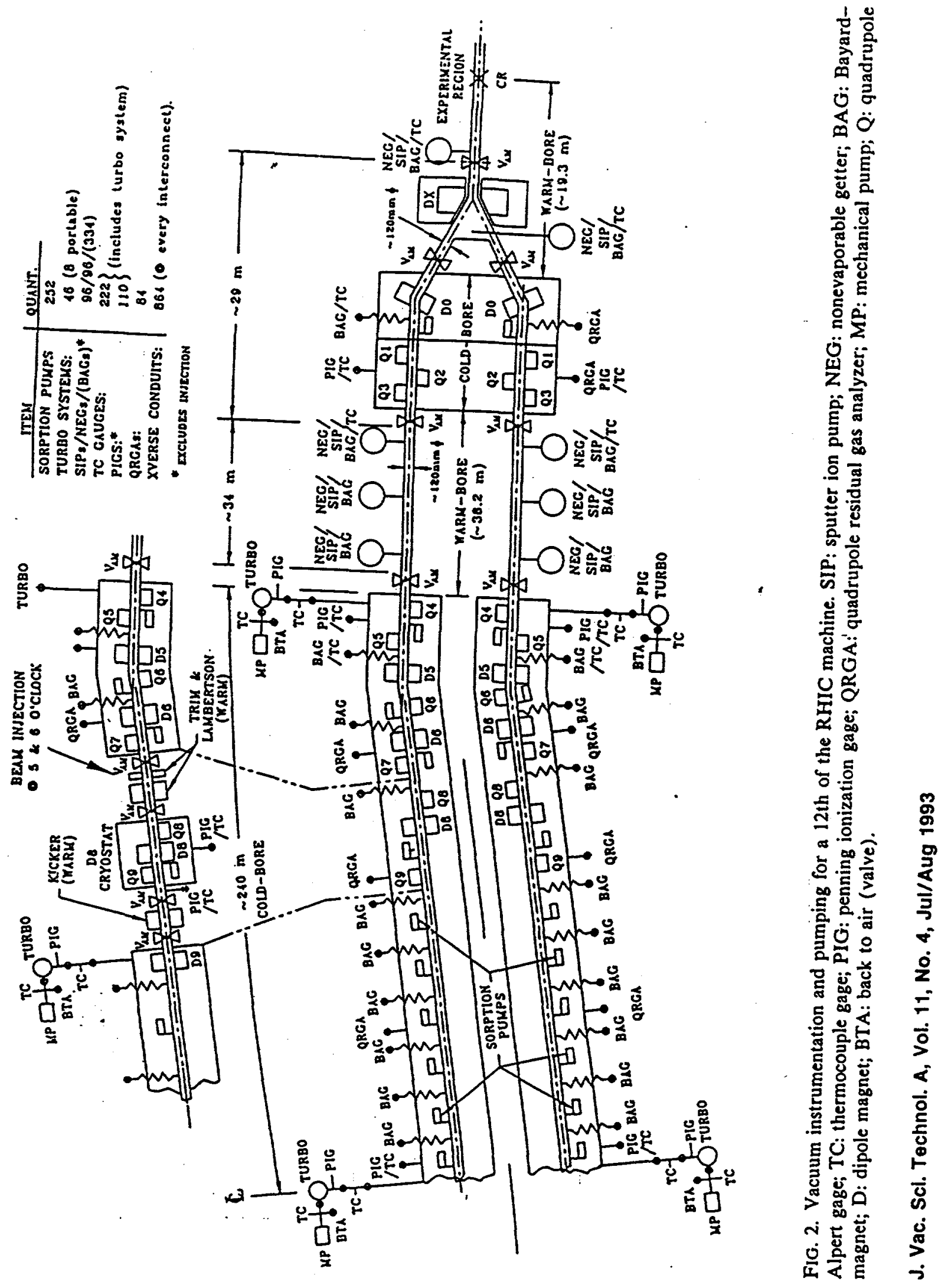




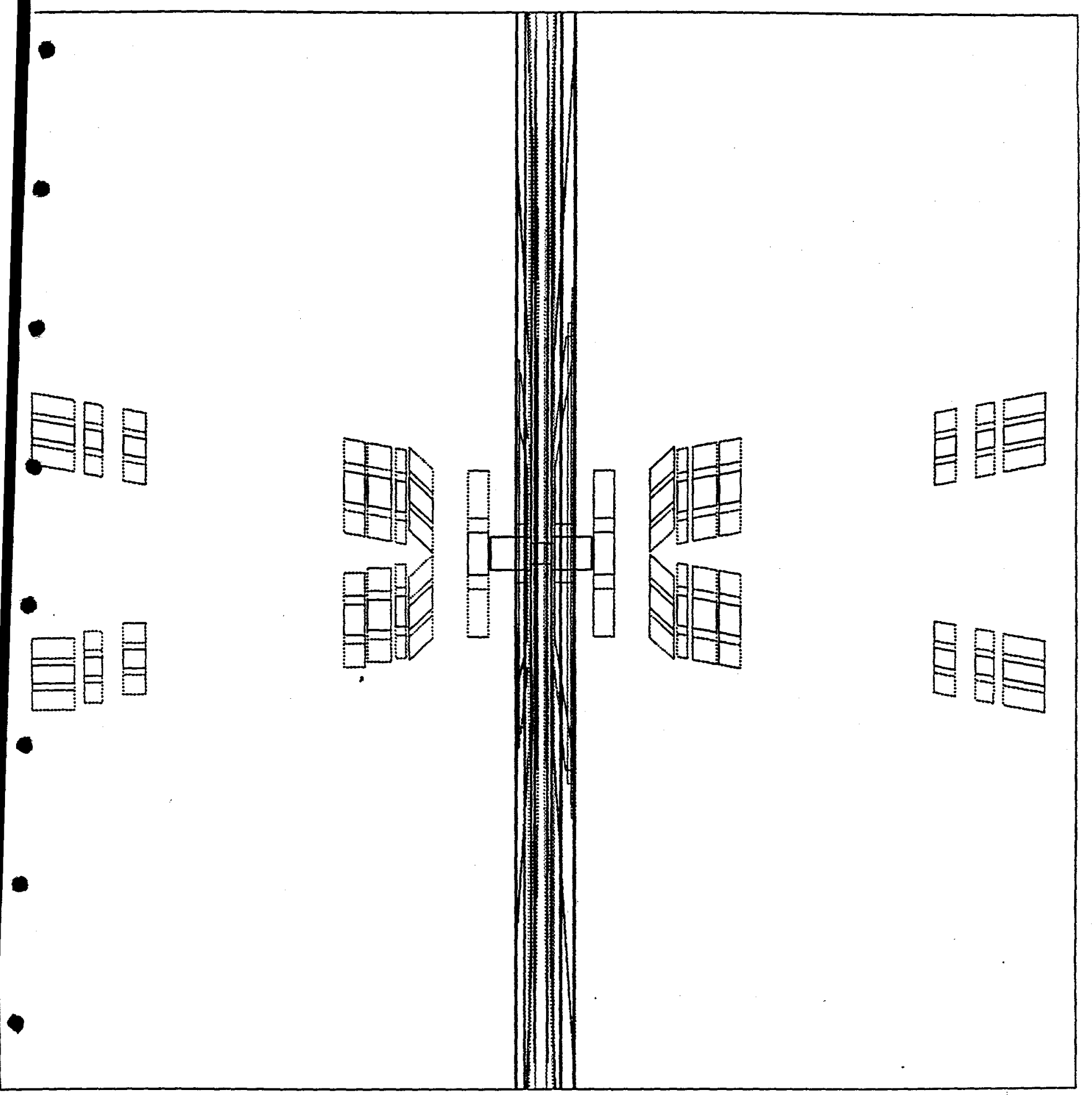


Special thanks to

Ron Lougacre

for the HIJET Giles! 


\title{
Number Distributions for Produced Particles
}

\author{
100 Au-p interactions \\ $12 \mathrm{Au}-\mathrm{N}$ interactions \\ 1 Au-He interaction
}

$\begin{array}{cc}\text { Particle } & \text { Mean Number per Interaction } \\ \gamma & 0.81 \\ \mathrm{e}^{+} & 0.009 \\ \mathrm{e}^{-} & 0.009 \\ \pi^{0} & 7.96 \\ \pi^{+} & 7.96 \\ \pi^{-} & 7.14 \\ \mathrm{~K}^{0} & 0.72 \\ \mathrm{~K} & 0.48 \\ \mathrm{~K} & 0.21 \\ \mathrm{n} & 118.2 \\ \mathrm{p} & 80.76 \\ \bar{p} & 0.009 \\ \bar{n} & 0.009 \\ \Lambda^{0} & 0.31 \\ \Sigma^{+} & 0.035 \\ \Sigma^{0} & 0.097 \\ \Sigma^{-} & 0.071 \\ \bar{\Sigma}^{-} & 0.009\end{array}$


Number Distributions for Pions
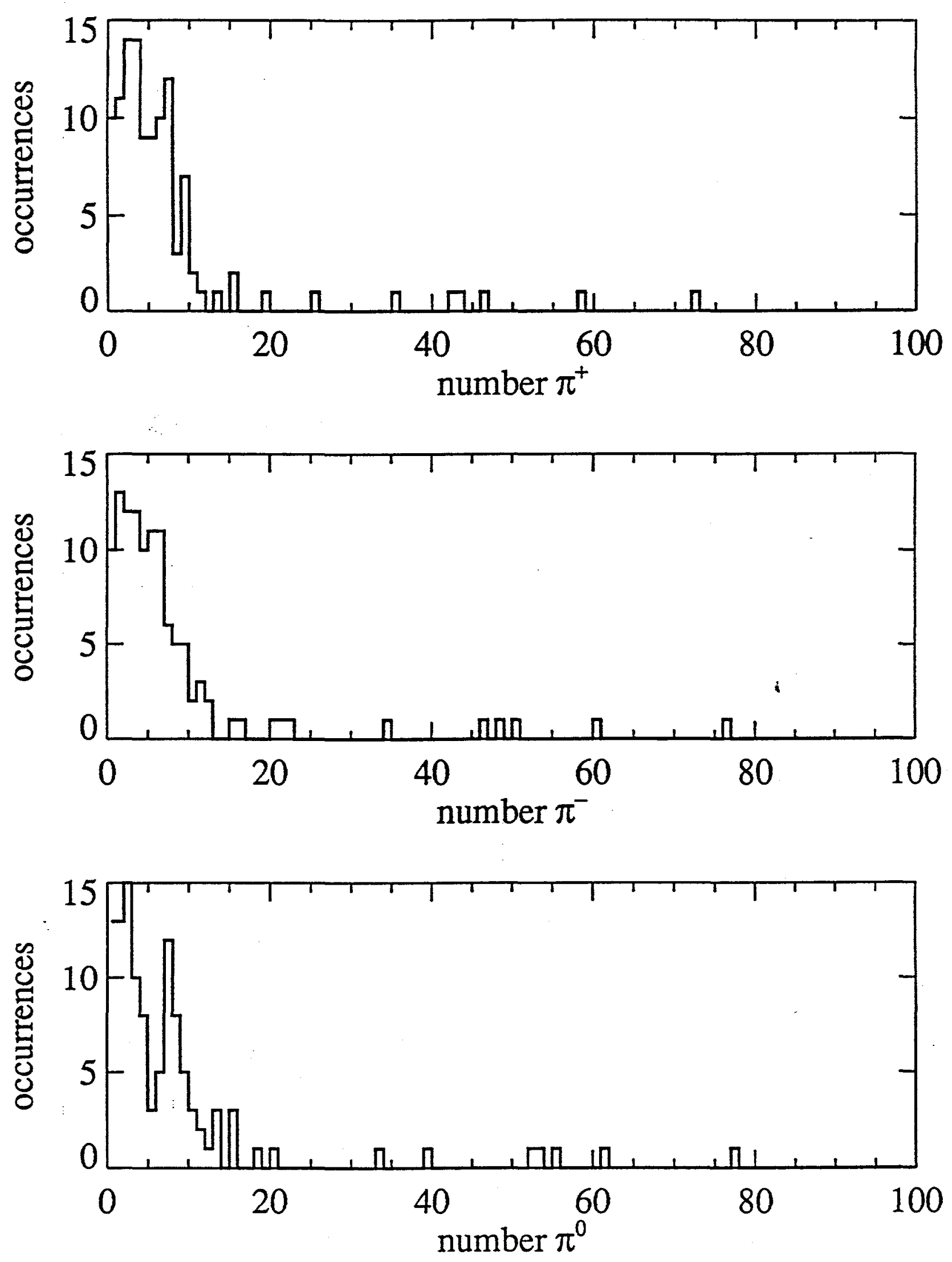


\section{Number Distributions for Kaons}
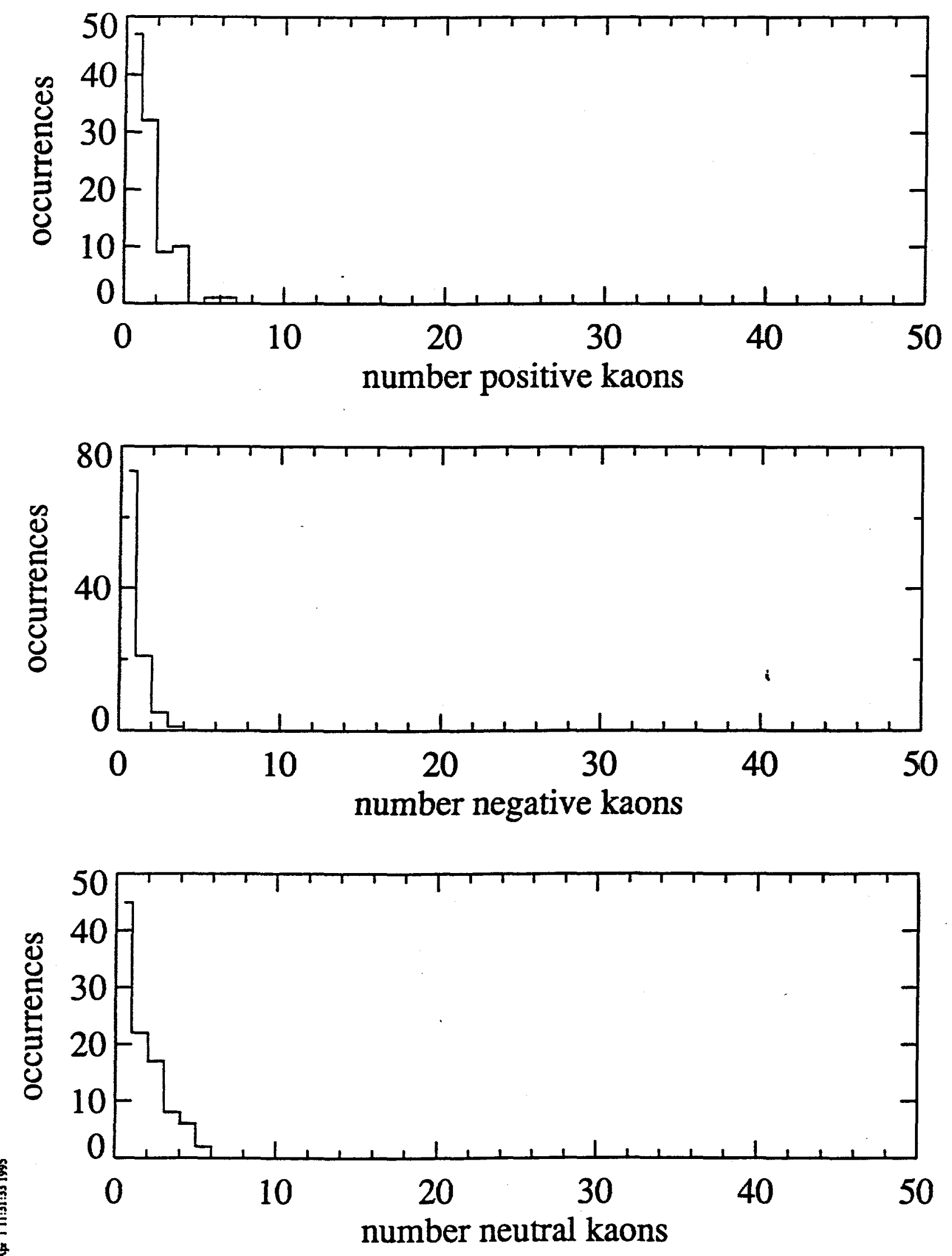
Number Distributions for Electrons and Positrons
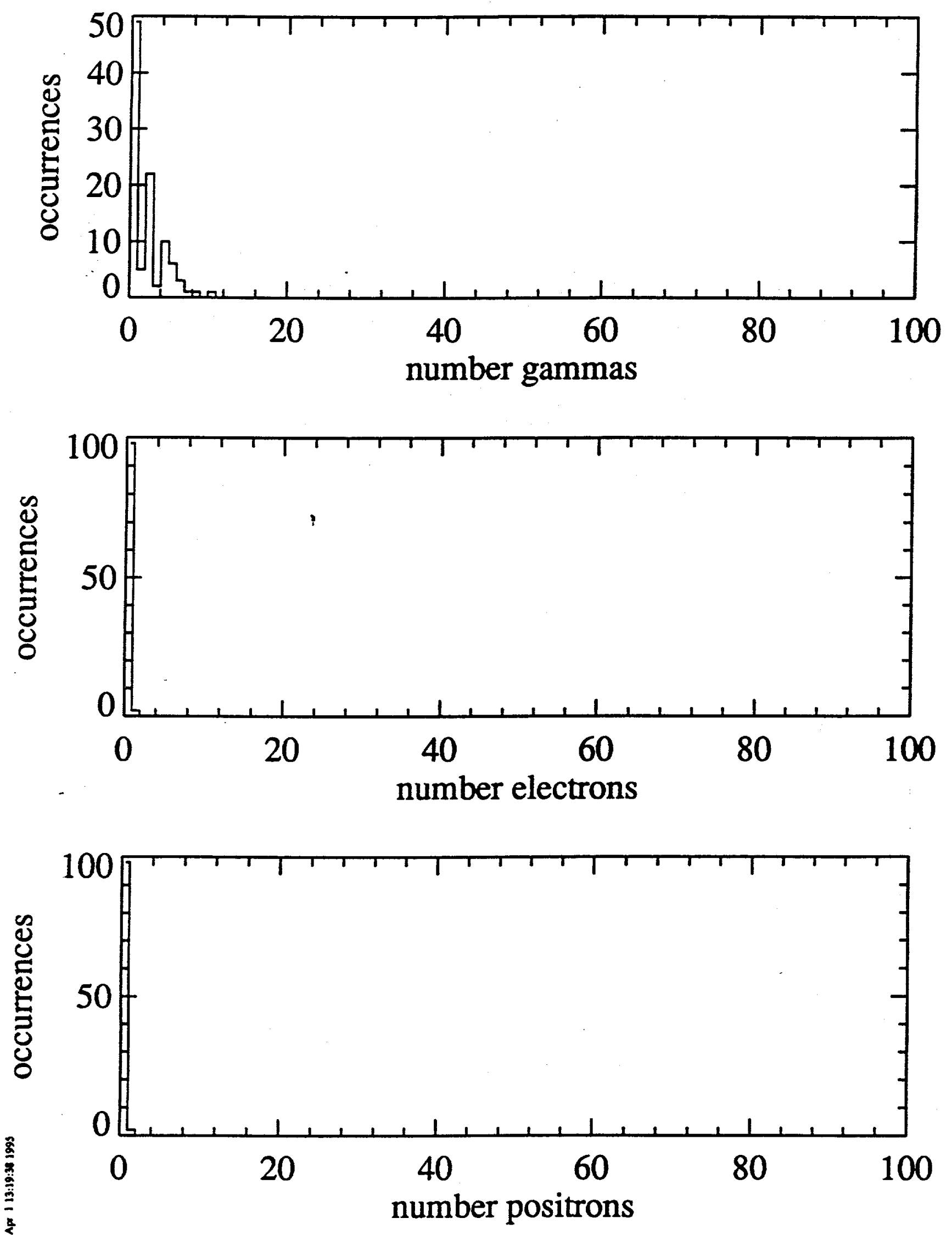


\section{Differential Distributions for $\pi^{0}$}
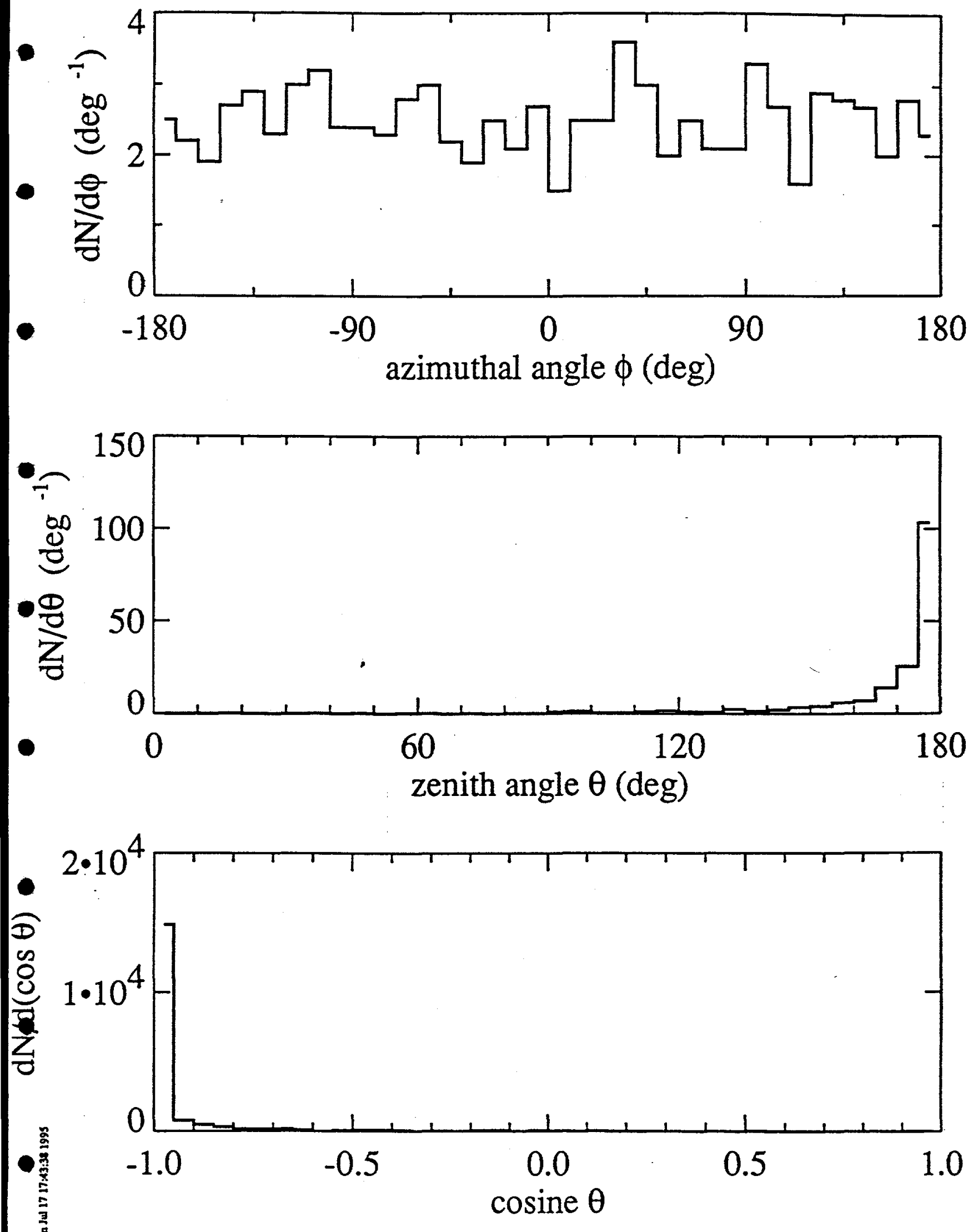


\section{Differential Distributions for $\pi^{0}$}
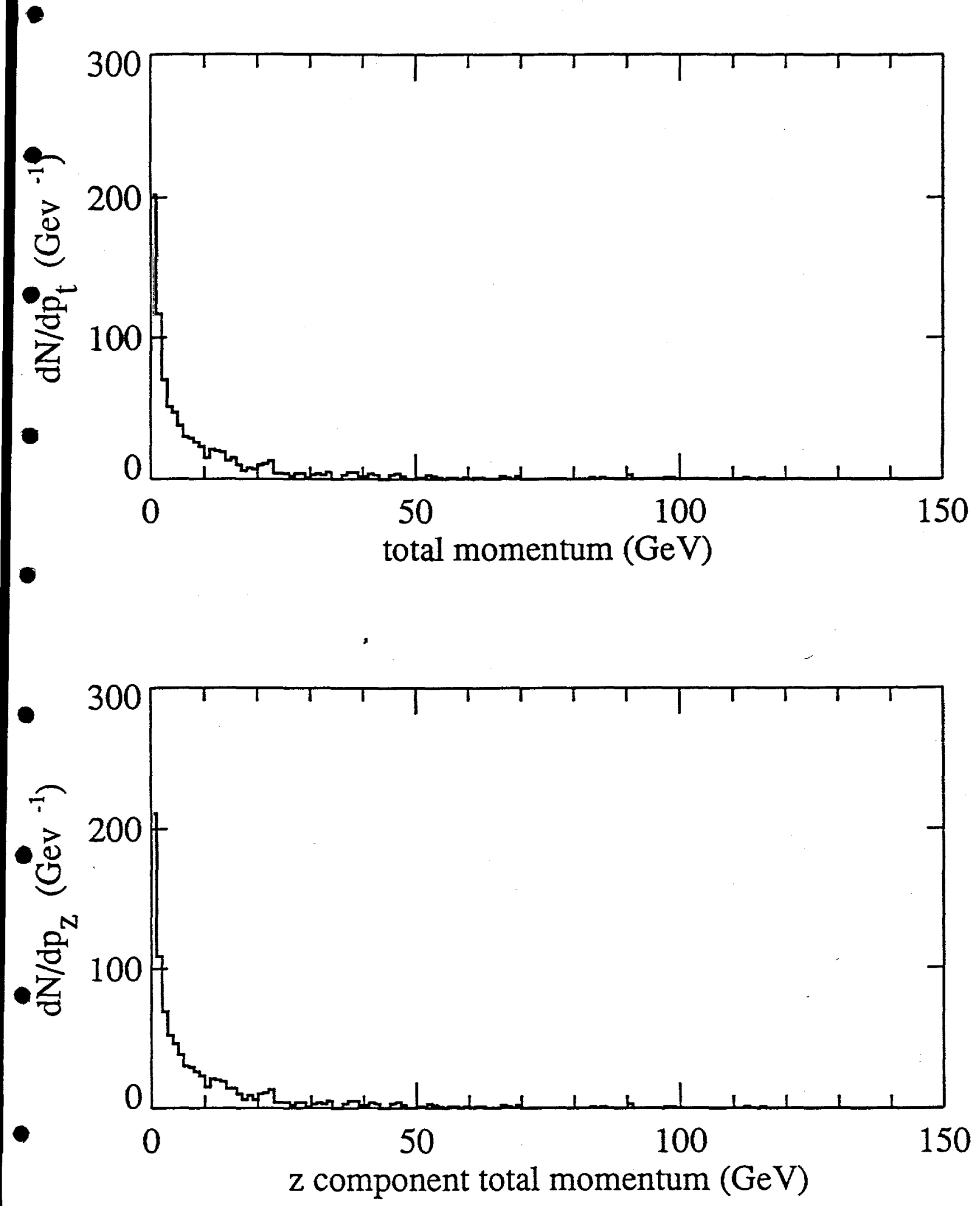


\section{Differential Distributions for $\pi^{+}$}
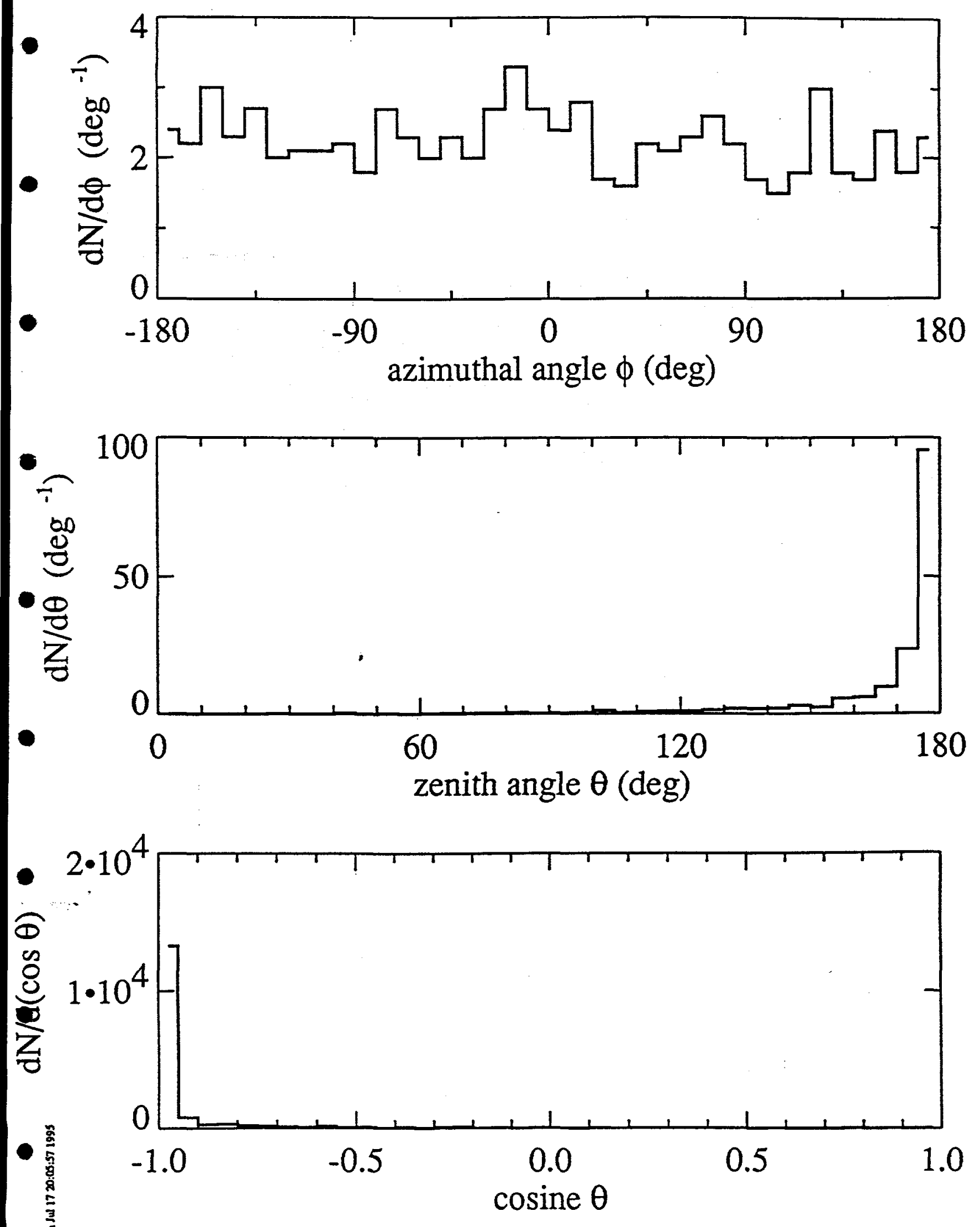


\section{Differential Distributions for $\pi^{+}$}
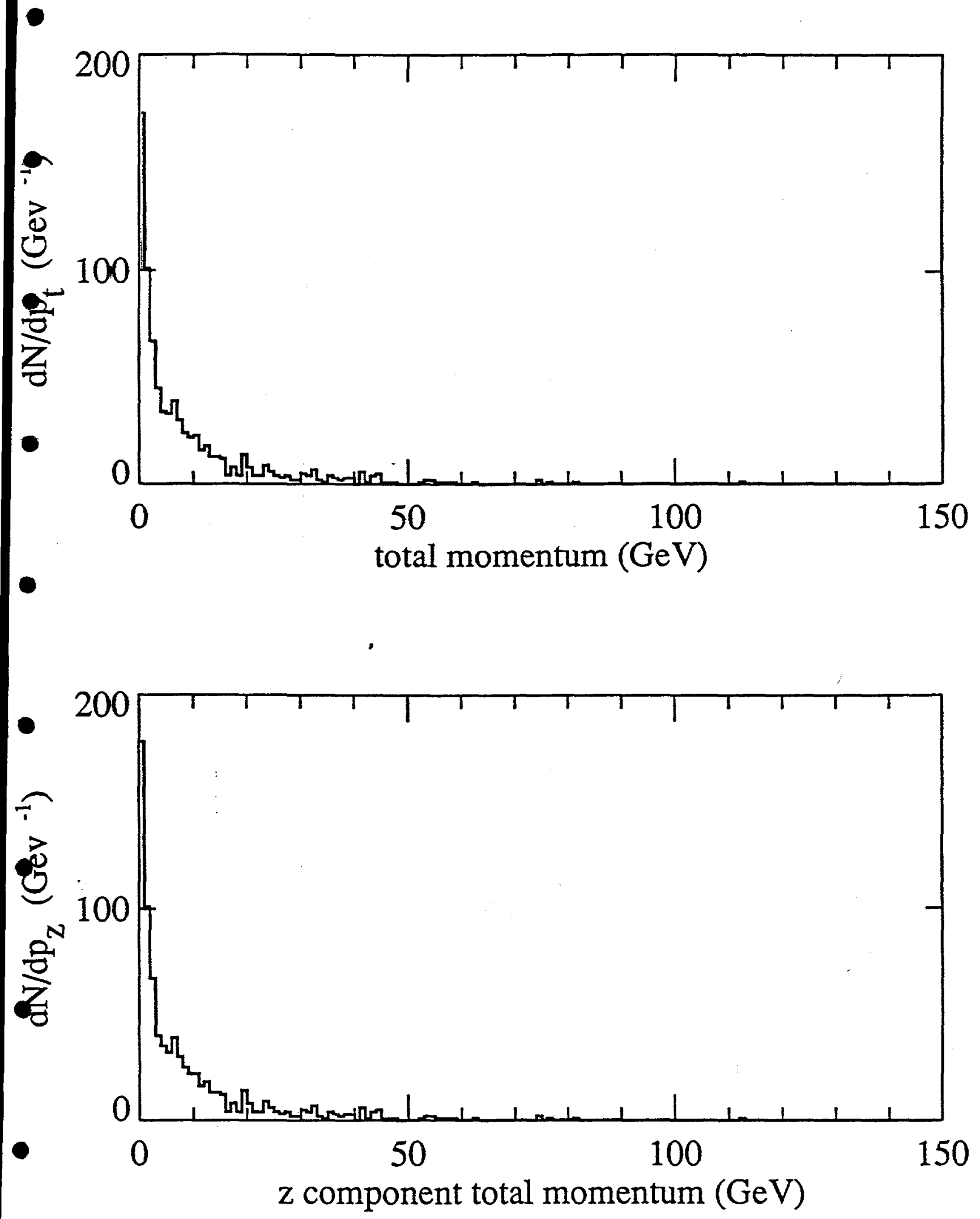


\section{Differential Distributions for $\pi^{-}$}
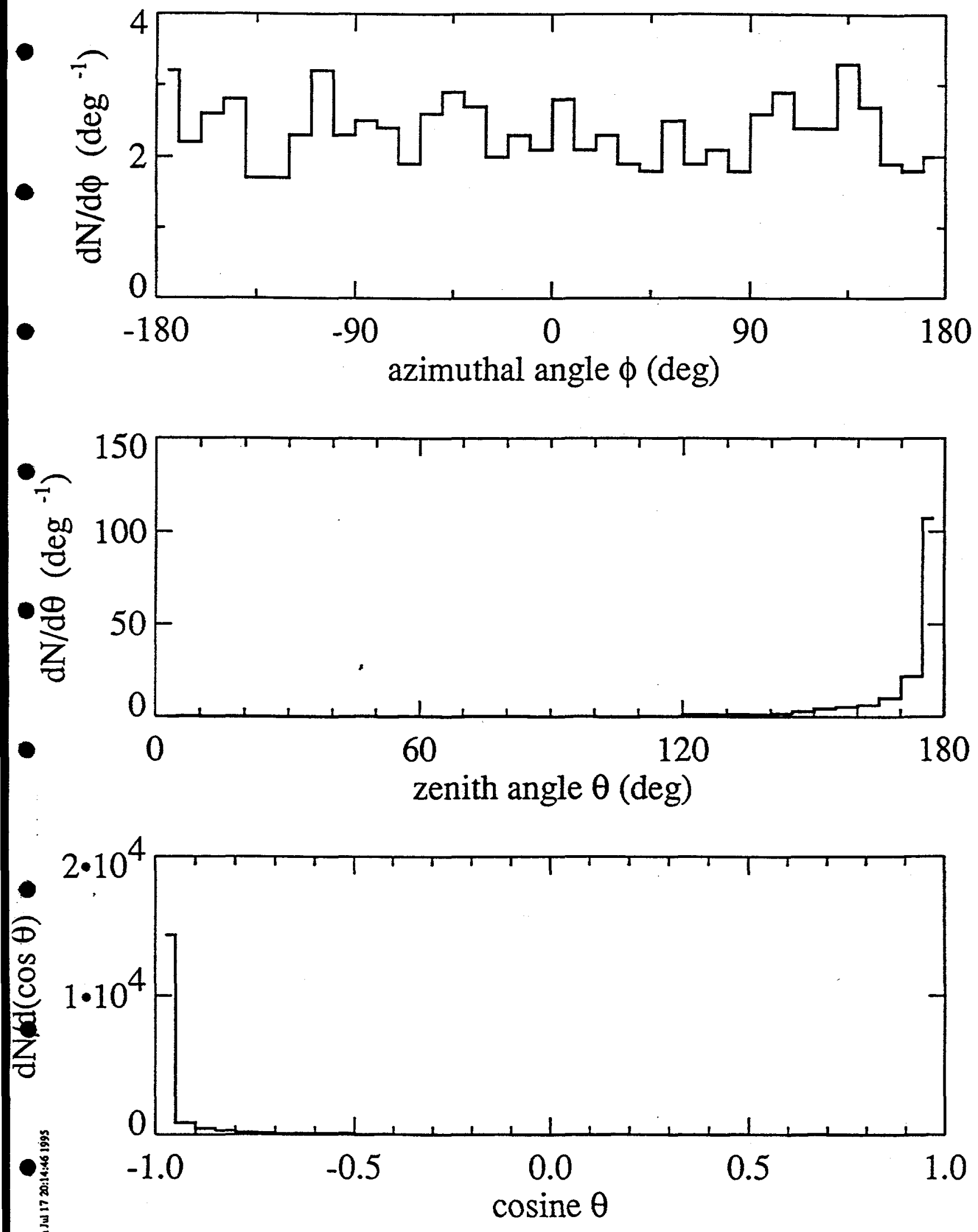


\section{Differential Distributions for $\pi^{-}$}
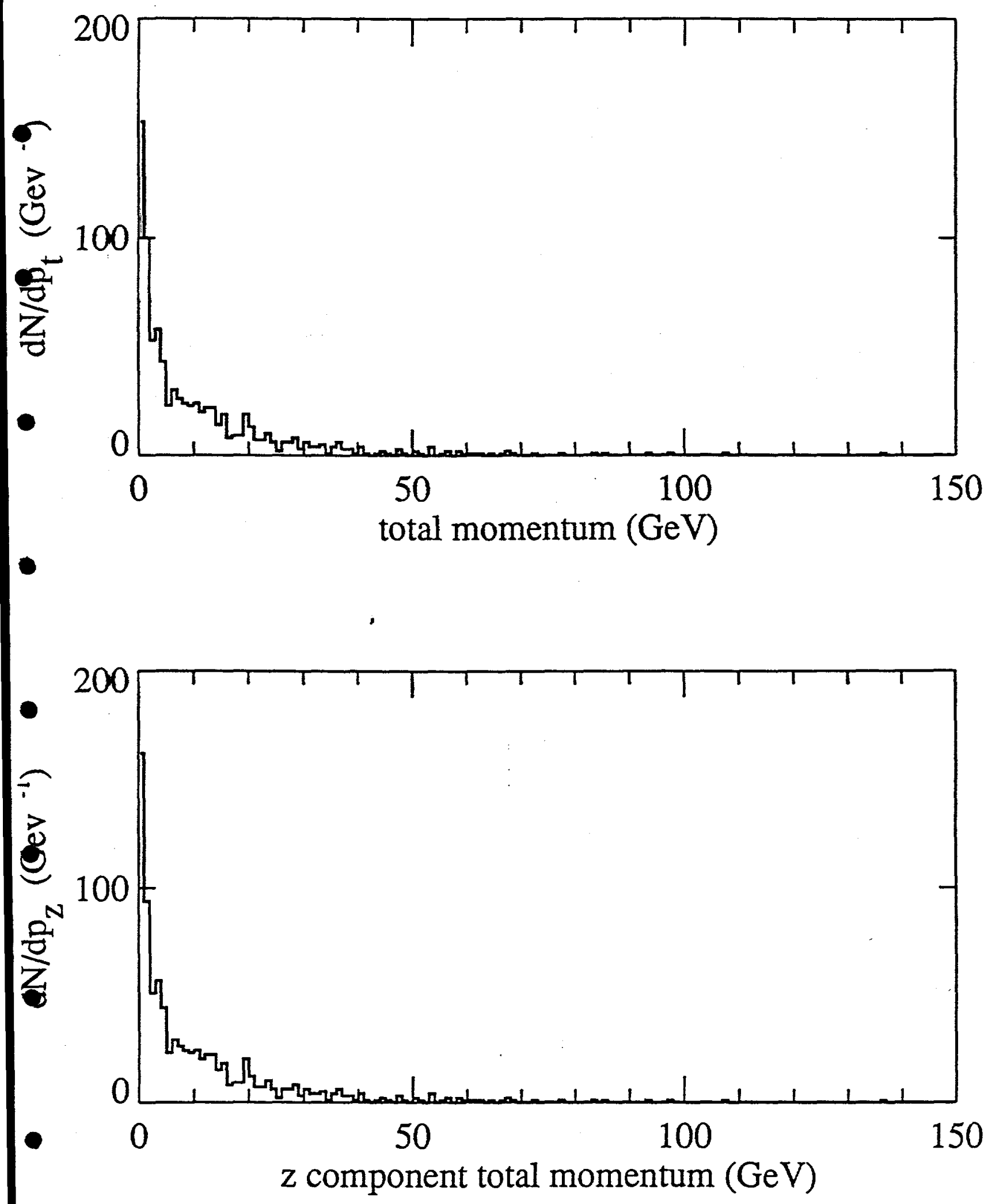
Differential Distributions for protons
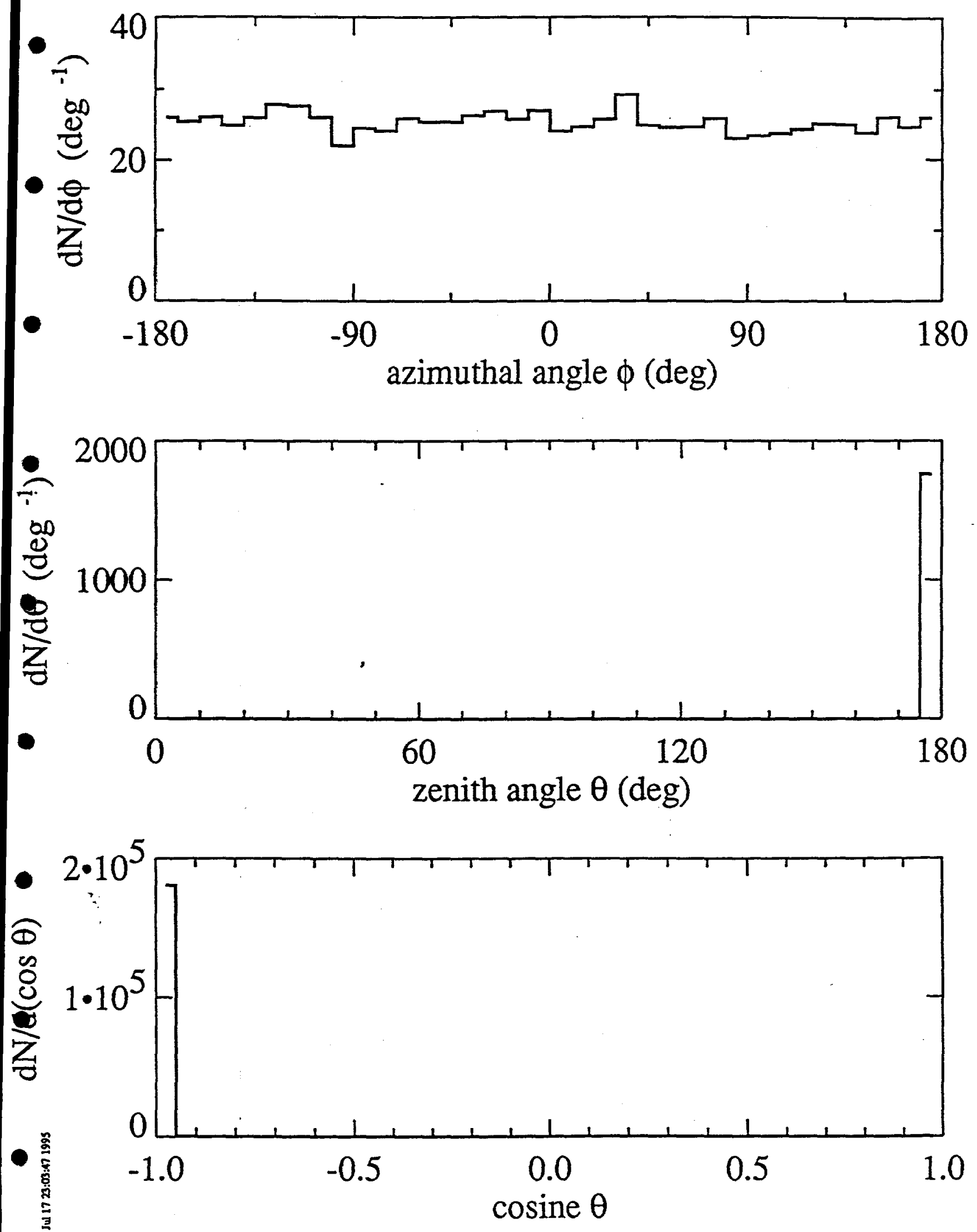


\section{Differential Distributions for neutrons}
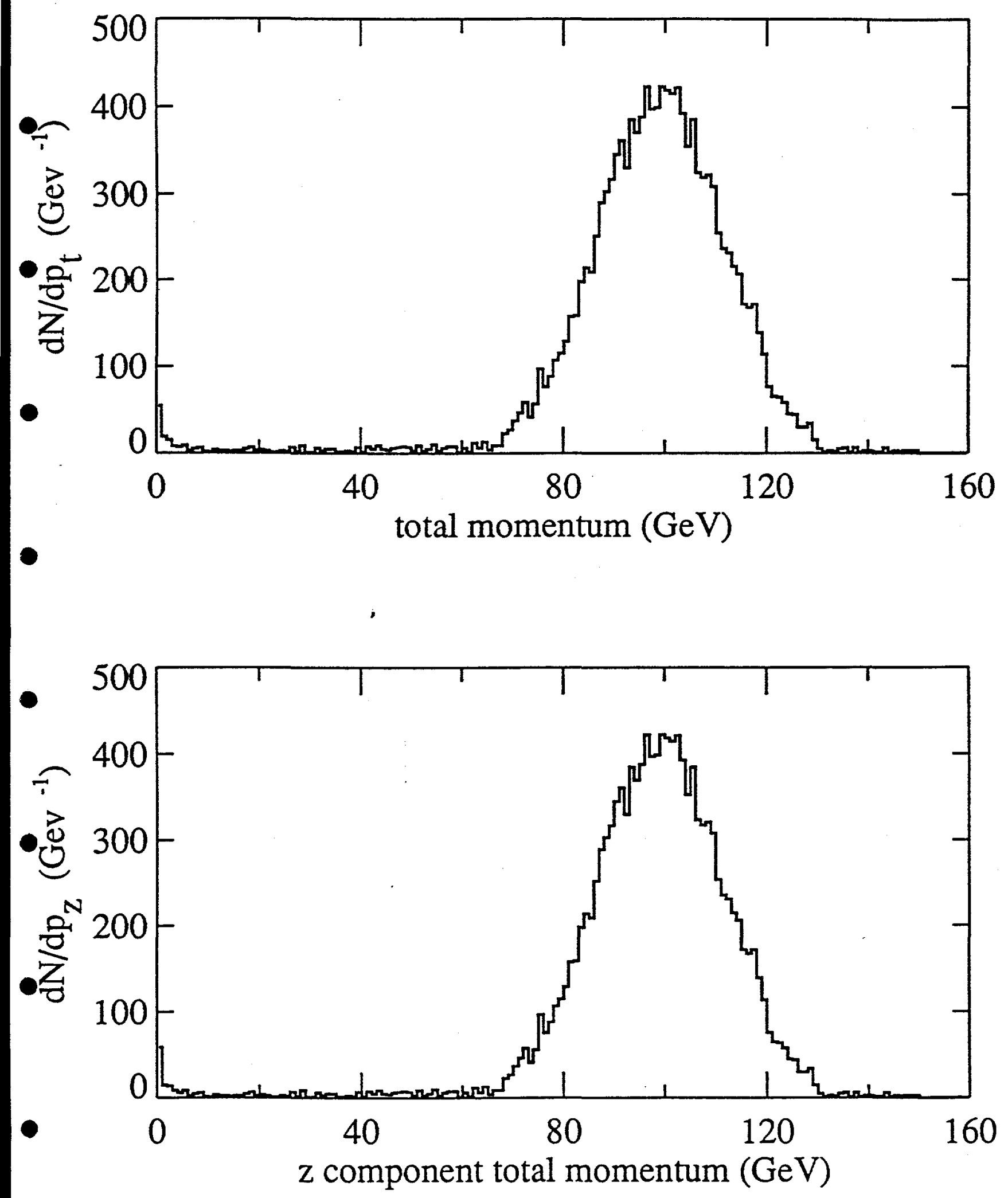
Effect on hevel-1 trigger

To date 20 events have been analyzed.

- 4,506 particles produced in initial Au-tanget collision -

19,284, changed particle hits recorded in muon id

aug. multiplicity $\approx \frac{19,284}{20 \cdot 6}=160$

160 hits/plaue / event !

but... level -1 trigger requires

2 on move tracks that satisfy

"road conditions 
- Effects-2-

- 6 of 20 events satisfy this - criterion ....

$30 \%$ of beam-gas interactions satisfy level-1 triggen 
What next?

Complete analysis of HIJET files numbers $\&$ differential distributions examination of neutrons study of events at $(-z)$ examine new geometry for detectors 
Appendix D 
$\overline{\text { Runs Through Geant For a Single Momentum }}$ 11/29/95

\begin{tabular}{cccccc}
\hline $\begin{array}{c}\text { Momentum } \\
(\mathrm{GeV})\end{array}$ & $\tilde{\boldsymbol{U}}$ & $\tilde{\boldsymbol{U}}$ & $\boldsymbol{\eta}$ & $\begin{array}{c}\text { Standard } \\
\text { deviation }\end{array}$ & $\begin{array}{c}\text { Initial } \\
\text { Ensemble }\end{array}$ \\
\hline $\mathbf{0 . 0 5 0}$ & $\mathbf{0 . 1 0}$ & & & & \\
& & 0.15 & 0.0004 & 0.0002 & 15000 \\
& 0.30 & 0.0004 & 0.0002 & 15000 \\
& 0.32 & 0.0006 & 0.0002 & 16000 \\
& 0.10 & & & & \\
& & 0.15 & 0.0005 & 0.0002 & 15000 \\
& & 0.30 & 0.0011 & 0.0003 & 15000 \\
& 0.32 & 0.0004 & 0.0002 & 16000 \\
& 0.12 & & & & 16000 \\
& & 0.15 & 0.0003 & 0.0001 & 15000 \\
& & 0.30 & 0.0003 & 0.0001 & 16000
\end{tabular}

Page: 1 
Momentum $(\mathrm{GeV})$

0.055
Standard Initial deviation Ensemble

$-0.12$

$-0.11$

$\begin{array}{rrrr}-0.15 & 0.0001 & 0.0001 & 15000 \\ 0.00 & 0.0005 & 0.0002 & 20000 \\ 0.05 & 0.0008 & 0.0002 & 20000 \\ 0.10 & 0.0022 & 0.0003 & 20000 \\ 0.15 & 0.0054 & 0.0007 & 12000 \\ 0.28 & 0.0087 & 0.0007 & 20000 \\ 0.30 & 0.0077 & 0.0008 & 12000 \\ 0.32 & 0.0064 & 0.0007 & 12000\end{array}$

$\begin{array}{llll}0.00 & 0.0007 & 0.0002 & 20000 \\ 0.05 & 0.0016 & 0.0003 & 20000 \\ 0.10 & 0.0039 & 0.0004 & 20000 \\ 0.15 & 0.0061 & 0.0005 & 20000 \\ 0.28 & 0.0105 & 0.0007 & 19680 \\ 0.30 & 0.0122 & 0.0008 & 20000 \\ 0.32 & 0.0088 & 0.0007 & 20000 \\ 0.34 & 0.0074 & 0.0006 & 20000 \\ 0.36 & 0.0046 & 0.0005 & 20000\end{array}$

$-0.10$

$\begin{array}{llll}0.00 & 0.0004 & 0.0001 & 20000 \\ 0.05 & 0.0019 & 0.0003 & 20000 \\ 0.10 & 0.0043 & 0.0005 & 20000 \\ 0.15 & 0.0071 & 0.0007 & 15000 \\ 0.28 & 0.0167 & 0.0009 & 20000 \\ 0.30 & 0.0146 & 0.0011 & 12000 \\ 0.32 & 0.0116 & 0.0010 & 12000 \\ 0.34 & 0.0105 & 0.0007 & 20000\end{array}$

Page: 2 
Momentum $(\mathrm{GeV})$

0.055

$\eta$

0.0074

0.0006

20000

$-0.05$

$\begin{array}{llll}0.00 & 0.0016 & 0.0003 & 20000 \\ 0.05 & 0.0033 & 0.0004 & 20000 \\ 0.10 & 0.0068 & 0.0006 & 20000 \\ 0.15 & 0.0138 & 0.0008 & 20000 \\ 0.28 & 0.0269 & 0.0012 & 19360 \\ 0.30 & 0.0289 & 0.0013 & 17120 \\ 0.32 & 0.0291 & 0.0012 & 18880 \\ 0.34 & 0.0348 & 0.0014 & 17600 \\ 0.36 & 0.0367 & 0.0013 & 20000 \\ 0.38 & 0.0417 & 0.0014 & 20000 \\ 0.40 & 0.0390 & 0.0014 & 20000 \\ 0.42 & 0.0243 & 0.0011 & 20000\end{array}$

0.00

$\begin{array}{llll}0.00 & 0.0013 & 0.0004 & 10000 \\ 0.05 & 0.0025 & 0.0004 & 20000 \\ 0.10 & 0.0068 & 0.0006 & 20000 \\ 0.15 & 0.0125 & 0.0010 & 12000 \\ 0.28 & 0.0269 & 0.0012 & 18240 \\ 0.30 & 0.0324 & 0.0016 & 12000 \\ 0.32 & 0.0369 & 0.0017 & 12000 \\ 0.34 & 0.0539 & 0.0020 & 12640 \\ 0.36 & 0.0622 & 0.0017 & 20000 \\ 0.38 & 0.0736 & 0.0018 & 20000 \\ 0.40 & 0.0724 & 0.0018 & 20000 \\ 0.42 & 0.0539 & 0.0016 & 20000 \\ 0.44 & 0.0219 & 0.0010 & 20000\end{array}$

Page: 3 


\section{Momentum}

$(\mathrm{GeV})$

\section{$\tilde{\mathbf{v}}$ \\ 0.05}

$\eta$

Standard Initial deviation Ensemble

$\begin{array}{llll}0.00 & 0.0015 & 0.0003 & 20000 \\ 0.05 & 0.0034 & 0.0004 & 20000 \\ 0.10 & 0.0057 & 0.0005 & 20000 \\ 0.15 & 0.0113 & 0.0007 & 20000 \\ 0.28 & 0.0233 & 0.0011 & 19840 \\ 0.30 & 0.0262 & 0.0012 & 18240 \\ 0.32 & 0.0264 & 0.0012 & 18560 \\ 0.34 & 0.0310 & 0.0013 & 18880 \\ 0.36 & 0.0321 & 0.0012 & 20000 \\ 0.38 & 0.0326 & 0.0013 & 20000 \\ 0.40 & 0.0292 & 0.0012 & 20000 \\ 0.42 & 0.0165 & 0.0009 & 20000\end{array}$

0.10

-0.15
0.00
0.05
0.10
0.15
0.28
0.30
0.32
0.34
0.36

0.0000

0.0000

20000

0.0011

0.0002

20000

0.0017

0.0003

20000

0.0040

0.0004

20000

0.0073

0.0008

12000

0.0130

0.0008

20000

0.0113

0.0010

12000

0.0088

0.0009

12000

0.0087

0.0007

20000

0.0053

0.0005

20000

\subsection{1}

$\begin{array}{llll}0.00 & 0.0005 & 0.0001 & 20000 \\ 0.05 & 0.0009 & 0.0002 & 20000 \\ 0.10 & 0.0025 & 0.0004 & 20000 \\ 0.15 & 0.0055 & 0.0005 & 20000\end{array}$

Page: 4 


\begin{tabular}{|c|c|c|c|c|c|}
\hline $\begin{array}{l}\text { Momentum } \\
(\mathrm{GeV})\end{array}$ & $\tilde{\boldsymbol{U}}$ & $\tilde{\boldsymbol{U}}$ & $\eta$ & $\begin{array}{l}\text { Standard } \\
\text { deviation }\end{array}$ & $\begin{array}{c}\text { Initial } \\
\text { Ensemble }\end{array}$ \\
\hline \multicolumn{6}{|l|}{0.055} \\
\hline & & 0.28 & 0.0099 & 0.0007 & 20000 \\
\hline & & 0.30 & 0.0093 & 0.0007 & 20000 \\
\hline & & 0.32 & 0.0071 & 0.0006 & 20000 \\
\hline & & 0.34 & 0.0059 & 0.0005 & 20000 \\
\hline \multicolumn{6}{|c|}{0.12} \\
\hline & & -0.15 & 0.0000 & 0.0000 & 20000 \\
\hline & & 0.00 & 0.0003 & 0.0001 & 20000 \\
\hline & & 0.05 & 0.0009 & 0.0002 & 20000 \\
\hline & & 0.10 & 0.0016 & 0.0003 & 20000 \\
\hline & 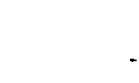 & 0.15 & 0.0038 & 0.0006 & 12000 \\
\hline & & 0.28 & 0.0065 & 0.0006 & 20000 \\
\hline & & 0.30 & 0.0062 & 0.0007 & 12000 \\
\hline & & 0.32 & 0.0054 & 0.0007 & 12000 \\
\hline & & 0.34 & 0.0032 & 0.0004 & 20000 \\
\hline
\end{tabular}

Page: 5 
Momentum $(\mathrm{GeV})$

0.060 $\eta$

Standard Initial deviation Ensemble

$-0.12$

$-\mathbf{0 . 1 0}$

$\begin{array}{lllr}0.00 & 0.0045 & 0.0007 & 10000 \\ 0.15 & 0.0362 & 0.0020 & 8500 \\ 0.30 & 0.0501 & 0.0024 & 8500 \\ 0.32 & 0.0379 & 0.0021 & 8500\end{array}$

0.00

$\begin{array}{lllr}0.00 & 0.0091 & 0.0009 & 10000 \\ 0.15 & 0.0561 & 0.0025 & 8500 \\ 0.30 & 0.0842 & 0.0030 & 8500 \\ 0.32 & 0.0656 & 0.0027 & 8500\end{array}$

$\begin{array}{llll}0.00 & 0.0128 & 0.0016 & 5000 \\ 0.15 & 0.0749 & 0.0029 & 8500 \\ 0.30 & 0.1514 & 0.0039 & 8500 \\ 0.32 & 0.1631 & 0.0040 & 8500\end{array}$

0.10

$\begin{array}{lllr}0.00 & 0.0073 & 0.0009 & 10000 \\ 0.15 & 0.0527 & 0.0024 & 8500 \\ 0.30 & 0.0740 & 0.0028 & 8500 \\ 0.32 & 0.0609 & 0.0026 & 8500\end{array}$

0.12

$\begin{array}{llll}0.00 & 0.0033 & 0.0006 & 10000\end{array}$




\begin{tabular}{cccccc}
\hline $\begin{array}{c}\text { Momentum } \\
(\mathrm{GeV})\end{array}$ & $\tilde{\mathbf{U}}$ & $\tilde{\mathbf{U}}$ & $\boldsymbol{\eta}$ & $\begin{array}{c}\text { Standard } \\
\text { deviation }\end{array}$ & $\begin{array}{c}\text { Initial } \\
\text { Ensemble }\end{array}$ \\
\hline $\mathbf{0 . 0 6 3}$ & & & & & \\
& -0.10 & -0.15 & 0.0005 & 0.0002 & 16000 \\
& & 0.00 & 0.0251 & 0.0013 & 15000 \\
& 0.15 & 0.1322 & 0.0027 & 16000 \\
& 0.00 & & & & \\
& & -0.15 & 0.0008 & 0.0002 & 16000 \\
& 0.00 & 0.0393 & 0.0016 & 15000 \\
& & 0.15 & 0.1634 & 0.0029 & 16000 \\
& 0.10 & & & & 16000 \\
& & 0.15 & 0.0006 & 0.0002 & 15000 \\
& & 0.00 & 0.0227 & 0.0012 & 16000
\end{tabular}




\begin{tabular}{crrrrr}
\hline $\begin{array}{c}\text { Momentum } \\
(\mathrm{GeV})\end{array}$ & $\tilde{\mathbf{U}}$ & $\tilde{\mathbf{U}}$ & $\boldsymbol{\eta}$ & $\begin{array}{c}\text { Standard } \\
\text { deviation }\end{array}$ & $\begin{array}{r}\text { Initial } \\
\text { Ensemble }\end{array}$ \\
\hline $\mathbf{0 . 0 6 5}$ & $\mathbf{0 . 1 2}$ & & & & \\
& & & & & \\
& -0.10 & 0.0030 & 0.0004 & 20000 \\
& -0.05 & 0.0134 & 0.0008 & 20000 \\
& 0.00 & 0.0261 & 0.0016 & 10000 \\
& 0.05 & 0.0505 & 0.0018 & 15520 \\
& 0.10 & 0.0826 & 0.0027 & 10720 \\
& 0.15 & 0.1160 & 0.0035 & 8500 \\
& 0.28 & 0.1419 & 0.0037 & 9107 \\
& 0.30 & 0.1325 & 0.0037 & 8500 \\
& 0.32 & 0.1068 & 0.0034 & 8500 \\
& 0.34 & 0.0923 & 0.0034 & 7136 \\
& 0.36 & 0.0673 & 0.0028 & 8000 \\
& 0.38 & 0.0437 & 0.0015 & 18000 \\
& 0.40 & 0.0204 & 0.0010 & 20000 \\
& 0.42 & 0.0051 & 0.0005 & 20000 \\
& 0.44 & 0.0014 & 0.0003 & 20000
\end{tabular}

$-0.11$

$\begin{array}{rrrr}-0.10 & 0.0050 & 0.0005 & 20000 \\ -0.05 & 0.0190 & 0.0010 & 19920 \\ 0.00 & 0.0415 & 0.0014 & 19000 \\ 0.05 & 0.0740 & 0.0020 & 18000 \\ 0.10 & 0.1108 & 0.0036 & 7644 \\ 0.15 & 0.1520 & 0.0036 & 9975 \\ 0.28 & 0.0000 & 0.0000 & 11757 \\ 0.30 & 0.1774 & 0.0036 & 11393 \\ 0.32 & 0.1537 & 0.0035 & 10588 \\ 0.34 & 0.1289 & 0.0035 & 9048 \\ 0.36 & 0.0989 & 0.0034 & 7707\end{array}$

Page: 8 
Momentum $(\mathrm{GeV})$

0.065 $\eta$

Standard Initial deviation Ensemble

$-0.10$

$\begin{array}{llll}0.38 & 0.0621 & 0.0020 & 15000 \\ 0.40 & 0.0300 & 0.0012 & 20000 \\ 0.42 & 0.0081 & 0.0006 & 20000 \\ 0.44 & 0.0019 & 0.0003 & 20000\end{array}$

$\begin{array}{rrrr}-0.15 & 0.0018 & 0.0004 & 10000 \\ -0.10 & 0.0076 & 0.0006 & 20000 \\ -0.05 & 0.0276 & 0.0012 & 18880 \\ 0.00 & 0.0490 & 0.0022 & 10000 \\ 0.05 & 0.0941 & 0.0033 & 7690 \\ 0.10 & 0.1402 & 0.0036 & 9107 \\ 0.15 & 0.1894 & 0.0047 & 7000 \\ 0.28 & 0.2281 & 0.0036 & 13226 \\ 0.30 & 0.2149 & 0.0049 & 7000 \\ 0.32 & 0.1992 & 0.0043 & 8500 \\ 0.34 & 0.1668 & 0.0035 & 11235 \\ 0.36 & 0.1310 & 0.0035 & 9400 \\ 0.38 & 0.0935 & 0.0029 & 10000 \\ 0.40 & 0.0467 & 0.0017 & 15000 \\ 0.42 & 0.0143 & 0.0008 & 20000 \\ 0.44 & 0.0030 & 0.0004 & 20000\end{array}$

$-0.05$

$\begin{array}{rrrr}-0.10 & 0.0119 & 0.0008 & 20000 \\ -0.05 & 0.0332 & 0.0013 & 19500 \\ 0.00 & 0.0708 & 0.0019 & 18000 \\ 0.05 & 0.1215 & 0.0034 & 8989 \\ 0.10 & 0.1820 & 0.0034 & 12848 \\ 0.15 & 0.2419 & 0.0036 & 14254\end{array}$

Page: 9 
Momentum $(\mathrm{GeV})$

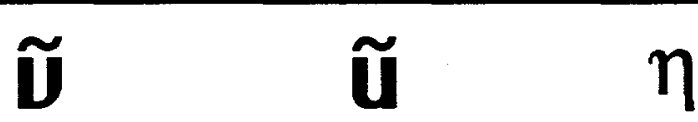

$\eta$

Standard deviation Ensemble

\subsection{5}

$\begin{array}{lllr}0.28 & 0.3155 & 0.0035 & 17232 \\ 0.30 & 0.3220 & 0.0035 & 17408 \\ 0.32 & 0.3297 & 0.0035 & 17550 \\ 0.34 & 0.3286 & 0.0035 & 17522 \\ 0.36 & 0.3198 & 0.0036 & 16927 \\ 0.38 & 0.2729 & 0.0035 & 16472 \\ 0.40 & 0.1928 & 0.0038 & 10643 \\ 0.42 & 0.1071 & 0.0034 & 8500 \\ 0.44 & 0.0305 & 0.0012 & 19800\end{array}$

0.00

$\begin{array}{rrrr}-0.15 & 0.0023 & 0.0006 & 7000 \\ -0.10 & 0.0131 & 0.0008 & 20000 \\ -0.05 & 0.0303 & 0.0013 & 18560 \\ 0.00 & 0.0672 & 0.0035 & 5000 \\ 0.05 & 0.1185 & 0.0038 & 7264 \\ 0.10 & 0.1788 & 0.0036 & 11446 \\ 0.15 & 0.2394 & 0.0051 & 7000 \\ 0.28 & 0.3311 & 0.0035 & 17688 \\ 0.30 & 0.3533 & 0.0057 & 7000 \\ 0.32 & 0.3754 & 0.0053 & 8500 \\ 0.34 & 0.4010 & 0.0035 & 19200 \\ 0.36 & 0.4108 & 0.0035 & 19475 \\ 0.38 & 0.3640 & 0.0035 & 18410 \\ 0.40 & 0.2787 & 0.0035 & 16302 \\ 0.42 & 0.1570 & 0.0035 & 10588 \\ 0.44 & 0.0520 & 0.0016 & 19500 \\ 0.45 & 0.0218 & 0.0010 & 20000\end{array}$

\subsection{5}

Page: 10 
Momentum $(\mathrm{GeV})$

0.065 $\eta$

0.0118

0.0330

0.0684

0.1119

0.1778

0.2375

0.3067

0.3040

0.3133

0.3075

0.2793

0.2412

0.1663

0.0883

0.0260
Standard Initial deviation Ensemble

0.10

-0.15
-0.10
-0.05
0.00
0.05
0.10
0.15
0.28
0.30
0.32
0.34
0.36

0.0006

0.0002

9000

0.0006

20000

0.0011

18400

0.0020

10000

0.0439

0.0024

12320

0.0034

9048

0.0040

9000

0.0035

13502

0.0043

8500

0.0040

8500

0.0028

16198

0.0030

10000

Page: 11 
Momentum $(\mathrm{GeV})$ 0.065 $\eta$

Standard Initial deviation Ensemble

$\begin{array}{llll}0.38 & 0.0735 & 0.0024 & 12000 \\ 0.40 & 0.0364 & 0.0014 & 19000 \\ 0.42 & 0.0097 & 0.0007 & 20000 \\ 0.44 & 0.0022 & 0.0003 & 20000\end{array}$

0.11

$\begin{array}{rrrr}-0.10 & 0.0050 & 0.0005 & 20000 \\ -0.05 & 0.0140 & 0.0008 & 20000 \\ 0.00 & 0.0330 & 0.0013 & 18400 \\ 0.05 & 0.0575 & 0.0021 & 12480 \\ 0.10 & 0.0943 & 0.0034 & 7360 \\ 0.15 & 0.1363 & 0.0036 & 9225 \\ 0.28 & 0.1653 & 0.0037 & 10312 \\ 0.30 & 0.1548 & 0.0035 & 10533 \\ 0.32 & 0.1356 & 0.0035 & 9574 \\ 0.34 & 0.1201 & 0.0036 & 8203 \\ 0.36 & 0.0764 & 0.0024 & 12000 \\ 0.38 & 0.0524 & 0.0020 & 12000 \\ 0.40 & 0.0231 & 0.0011 & 19000 \\ 0.42 & 0.0059 & 0.0005 & 20000 \\ 0.44 & 0.0015 & 0.0003 & 20000\end{array}$

0.12

$\begin{array}{rrrr}-0.10 & 0.0023 & 0.0003 & 20000 \\ -0.05 & 0.0099 & 0.0007 & 20000 \\ 0.00 & 0.0194 & 0.0014 & 9500 \\ 0.03 & 0.0327 & 0.0018 & 9500 \\ 0.05 & 0.0361 & 0.0014 & 17280 \\ 0.10 & 0.0621 & 0.0022 & 12160 \\ 0.15 & 0.0986 & 0.0031 & 9500\end{array}$

Page: 12 


\begin{tabular}{|c|c|c|c|c|}
\hline $\begin{array}{l}\text { Momentum } \\
(\mathrm{GeV})\end{array}$ & $\tilde{\mathbf{U}}$ & $\eta$ & $\begin{array}{l}\text { Standard } \\
\text { deviation }\end{array}$ & $\begin{array}{c}\text { Initial } \\
\text { Ensemble }\end{array}$ \\
\hline \multicolumn{5}{|l|}{0.065} \\
\hline & 0.28 & 0.1104 & 0.0035 & 8142 \\
\hline & 0.30 & 0.1094 & 0.0036 & 7680 \\
\hline & 0.32 & 0.0940 & 0.0030 & 9300 \\
\hline & 0.32 & 0.0935 & 0.0030 & 9500 \\
\hline & 0.34 & 0.0724 & 0.0026 & 10000 \\
\hline & 0.36 & 0.0513 & 0.0018 & 15000 \\
\hline & 0.38 & 0.0313 & 0.0013 & 18000 \\
\hline & 0.40 & 0.0154 & 0.0009 & 20000 \\
\hline & 0.42 & 0.0032 & 0.0004 & 20000 \\
\hline & 0.44 & 0.0006 & 0.0002 & 20000 \\
\hline
\end{tabular}

Page: 13 
Momentum $(\mathrm{GeV})$

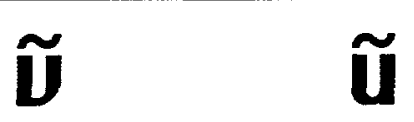

0.075 $\eta$

Standard deviation Ensemble

$-0.12$

$\begin{array}{rrrr}-0.15 & 0.0097 & 0.0010 & 10000 \\ -0.10 & 0.0712 & 0.0022 & 13600 \\ -0.05 & 0.1389 & 0.0036 & 8989 \\ 0.00 & 0.2030 & 0.0057 & 5000 \\ 0.05 & 0.2674 & 0.0034 & 17082 \\ 0.10 & 0.3114 & 0.0035 & 17379 \\ 0.15 & 0.3530 & 0.0048 & 10000 \\ 0.28 & 0.3384 & 0.0036 & 17688 \\ 0.30 & 0.3104 & 0.0055 & 7000 \\ 0.32 & 0.2886 & 0.0054 & 7000 \\ 0.34 & 0.2431 & 0.0035 & 14879 \\ 0.36 & 0.1877 & 0.0035 & 12411 \\ 0.38 & 0.1272 & 0.0035 & 9224 \\ 0.40 & 0.0559 & 0.0018 & 16000 \\ 0.42 & 0.0184 & 0.0010 & 20000 \\ 0.44 & 0.0048 & 0.0005 & 20000\end{array}$

$-0.11$

$\begin{array}{rrrr}-0.15 & 0.0148 & 0.0009 & 20000 \\ -0.10 & 0.1078 & 0.0035 & 7707 \\ -0.05 & 0.1963 & 0.0035 & 12629 \\ 0.00 & 0.2851 & 0.0036 & 15841 \\ 0.05 & 0.3609 & 0.0036 & 17822 \\ 0.10 & 0.4234 & 0.0035 & 19662 \\ 0.15 & 0.4684 & 0.0035 & 19831 \\ 0.28 & 0.4645 & 0.0035 & 19896 \\ 0.30 & 0.4408 & 0.0036 & 19475 \\ 0.32 & 0.4031 & 0.0035 & 19135\end{array}$

Page: 14 
Momentum (GeV)

0.075 $\eta$

Standard Initial deviation Ensemble

$-0.10$

$\begin{array}{rrrr}0.34 & 0.3489 & 0.0036 & 17874 \\ 0.36 & 0.2664 & 0.0035 & 15582 \\ 0.38 & 0.1835 & 0.0035 & 12112 \\ 0.40 & 0.0929 & 0.0031 & 9000 \\ 0.42 & 0.0295 & 0.0012 & 19700 \\ 0.44 & 0.0057 & 0.0005 & 20000\end{array}$

$\begin{array}{rrrr}-0.15 & 0.0230 & 0.0015 & 10000 \\ -0.10 & 0.1401 & 0.0034 & 10533 \\ -0.05 & 0.2461 & 0.0034 & 16163 \\ 0.00 & 0.3494 & 0.0048 & 10000 \\ 0.05 & 0.4391 & 0.0035 & 19608 \\ 0.10 & 0.5048 & 0.0035 & 19999 \\ 0.15 & 0.5523 & 0.0050 & 10000 \\ 0.28 & 0.5563 & 0.0035 & 19692 \\ 0.30 & 0.5320 & 0.0060 & 7000 \\ 0.32 & 0.4790 & 0.0060 & 7000 \\ 0.34 & 0.4286 & 0.0035 & 19619 \\ 0.36 & 0.3417 & 0.0035 & 18410 \\ 0.38 & 0.2291 & 0.0030 & 19692 \\ 0.40 & 0.1195 & 0.0035 & 8509 \\ 0.42 & 0.0408 & 0.0014 & 19500 \\ 0.44 & 0.0072 & 0.0006 & 20000\end{array}$

$-0.05$

$\begin{array}{rlll}-0.15 & 0.0355 & 0.0014 & 18472 \\ -0.10 & 0.1847 & 0.0035 & 12607 \\ -0.05 & 0.3001 & 0.0036 & 15878 \\ 0.00 & 0.4242 & 0.0036 & 19323\end{array}$

Page: 15 
Momentum $(\mathrm{GeV})$

Standard Initial

0.075

$\begin{array}{llll}0.05 & 0.5171 & 0.0035 & 19974 \\ 0.10 & 0.5948 & 0.0035 & 19475 \\ 0.15 & 0.6495 & 0.0035 & 18200 \\ 0.28 & 0.6724 & 0.0036 & 16958 \\ 0.30 & 0.6762 & 0.0036 & 17321 \\ 0.32 & 0.6776 & 0.0036 & 17262 \\ 0.34 & 0.6520 & 0.0036 & 17978 \\ 0.36 & 0.5745 & 0.0036 & 19263 \\ 0.38 & 0.4395 & 0.0035 & 19641 \\ 0.40 & 0.2859 & 0.0035 & 16268 \\ 0.42 & 0.1383 & 0.0034 & 10312 \\ 0.44 & 0.0351 & 0.0013 & 19800\end{array}$

0.00

$\begin{array}{rrrr}-0.15 & 0.0361 & 0.0019 & 10000 \\ -0.10 & 0.1756 & 0.0036 & 11235 \\ -0.05 & 0.2865 & 0.0035 & 16605 \\ 0.00 & 0.4090 & 0.0035 & 19538 \\ 0.05 & 0.5011 & 0.0035 & 20000 \\ 0.10 & 0.5787 & 0.0035 & 19585 \\ 0.15 & 0.6288 & 0.0048 & 10000 \\ 0.28 & 0.6841 & 0.0036 & 16638 \\ 0.30 & 0.6873 & 0.0055 & 7000 \\ 0.32 & 0.7031 & 0.0055 & 7000 \\ 0.34 & 0.6910 & 0.0036 & 16671 \\ 0.36 & 0.6486 & 0.0035 & 18127 \\ 0.38 & 0.5131 & 0.0035 & 20000 \\ 0.40 & 0.3473 & 0.0035 & 18271 \\ 0.42 & 0.1832 & 0.0037 & 11182\end{array}$

Page: 16 
Momentum $(\mathrm{GeV})$

$\tilde{\mathbf{v}}$

$\tilde{\mathbf{u}}$

$\eta$

0.075

$\begin{array}{llll}0.44 & 0.0530 & 0.0016 & 19500 \\ 0.45 & 0.0202 & 0.0010 & 20000\end{array}$

0.05

Standard Initial deviation Ensemble

$$
-0.1
$$

$-0.15$

0.0330

0.0013

18920

$-0.10$

0.1827

0.0035

12362

$-0.05$

0.2977

0.0036

15914

0.00

0.4210

0.0035

19394

0.05

0.5136

0.0035

19999

0.10

0.5877

0.0035

19263

0.15

0.6395

0.0035

18410

0.28

0.6686

0.0036

17522

0.30

0.6593

0.32

0.6606

0.0035

18476

0.34

0.6204

0.0035

18127

0.36

0.5416

0.38

0.4121

0.0035

18770

0.0035

19896

0.40

0.2594

0.0036

19168

0.42

0.1238

0.0035

15768

0.44

0.0297

0.0035

8989

0.0012

19800

0.10

$\begin{array}{rrrr}-0.15 & 0.0174 & 0.0013 & 10000 \\ -0.10 & 0.1219 & 0.0034 & 9166 \\ -0.05 & 0.2148 & 0.0036 & 13364 \\ 0.00 & 0.3023 & 0.0046 & 10000 \\ 0.05 & 0.3861 & 0.0035 & 19067 \\ 0.10 & 0.4673 & 0.0035 & 19974 \\ 0.15 & 0.5238 & 0.0050 & 10000 \\ 0.28 & 0.5120 & 0.0035 & 19971\end{array}$

Page: 17 
Momentum $(\mathrm{GeV})$

$\tilde{\mathbf{v}}$

$\tilde{\mathbf{u}}$

$\eta$

0.075
Standard Initial deviation Ensemble

$\begin{array}{lllr}0.30 & 0.4907 & 0.0060 & 7000 \\ 0.32 & 0.4466 & 0.0059 & 7000 \\ 0.34 & 0.3890 & 0.0035 & 19014 \\ 0.36 & 0.3021 & 0.0035 & 17350 \\ 0.38 & 0.1962 & 0.0036 & 12012 \\ 0.40 & 0.0952 & 0.0034 & 7455 \\ 0.42 & 0.0316 & 0.0013 & 19500 \\ 0.44 & 0.0067 & 0.0006 & 20000\end{array}$

0.11

$\begin{array}{rrrr}-0.15 & 0.0113 & 0.0007 & 20000 \\ -0.10 & 0.0822 & 0.0030 & 8480 \\ -0.05 & 0.1592 & 0.0036 & 10533 \\ 0.00 & 0.2352 & 0.0036 & 14254 \\ 0.05 & 0.3034 & 0.0035 & 16832 \\ 0.10 & 0.3602 & 0.0035 & 18318 \\ 0.15 & 0.4078 & 0.0035 & 19767 \\ 0.28 & 0.3996 & 0.0035 & 19366 \\ 0.30 & 0.3838 & 0.0035 & 18996 \\ 0.32 & 0.3536 & 0.0035 & 18175 \\ 0.34 & 0.3012 & 0.0036 & 16605 \\ 0.36 & 0.2348 & 0.0035 & 14675 \\ 0.38 & 0.1505 & 0.0035 & 10698 \\ 0.40 & 0.0719 & 0.0026 & 10000 \\ 0.42 & 0.0199 & 0.0010 & 20000 \\ 0.44 & 0.0042 & 0.0005 & 20000\end{array}$

0.12

$\begin{array}{rrrr}-0.15 & 0.0063 & 0.0009 & 7000 \\ -0.10 & 0.0488 & 0.0018 & 14400\end{array}$

Page: 18 
Momentum (GeV)

Standard

Initial

0.075

0.0971

0.0035

7200

0.00

0.1464

0.0050

5000

0.05

0.2006

0.0034

0.2435

0.10

0.2737

0.15

0.2760

0.28

0.30

0.2529

0.32

0.2351

0.34

0.2045

0.36

0.1502

0.38

0.40

0.42

0.44

0.1007

0.0459

0.0118

0.0027

0.0035

0.0045

0.0036

0.0052

0.0051

0.0036

0.0037

0.0030

0.0016

0.0008

0.0004
13593

15000

10000

15694

7000

7000

12703

9516

10000

18000

20000

20000

Page: 19 
Momentum (GeV)

0.085
Standard Initial deviation Ensemble

$-0.12$

$\begin{array}{rrrr}-0.30 & 0.0003 & 0.0002 & 7500 \\ -0.15 & 0.0864 & 0.0051 & 2998 \\ -0.10 & 0.2482 & 0.0038 & 13014 \\ -0.05 & 0.3226 & 0.0036 & 16605 \\ 0.00 & 0.3871 & 0.0049 & 10000 \\ 0.05 & 0.4287 & 0.0035 & 19816 \\ 0.10 & 0.4585 & 0.0035 & 19923 \\ 0.15 & 0.4768 & 0.0035 & 19866 \\ 0.28 & 0.4383 & 0.0036 & 19488 \\ 0.30 & 0.4244 & 0.0036 & 19101 \\ 0.32 & 0.3896 & 0.0036 & 18409 \\ 0.34 & 0.3388 & 0.0035 & 17822 \\ 0.36 & 0.2684 & 0.0036 & 15040 \\ 0.38 & 0.1836 & 0.0035 & 12312 \\ 0.40 & 0.0974 & 0.0032 & 8500 \\ 0.42 & 0.0291 & 0.0012 & 20000 \\ 0.44 & 0.0048 & 0.0005 & 20000\end{array}$

$-0.11$

$\begin{array}{rlll}-0.30 & 0.0002 & 0.0001 & 20000 \\ -0.15 & 0.1343 & 0.0034 & 10256 \\ -0.10 & 0.3785 & 0.0036 & 18432 \\ -0.05 & 0.4734 & 0.0035 & 19884 \\ 0.00 & 0.5475 & 0.0035 & 19672 \\ 0.05 & 0.6031 & 0.0035 & 19151 \\ 0.10 & 0.6260 & 0.0035 & 18770 \\ 0.15 & 0.6495 & 0.0035 & 18318 \\ 0.28 & 0.6111 & 0.0035 & 19135\end{array}$

Page: 20 
Momentum $(\mathrm{GeV})$

0.085

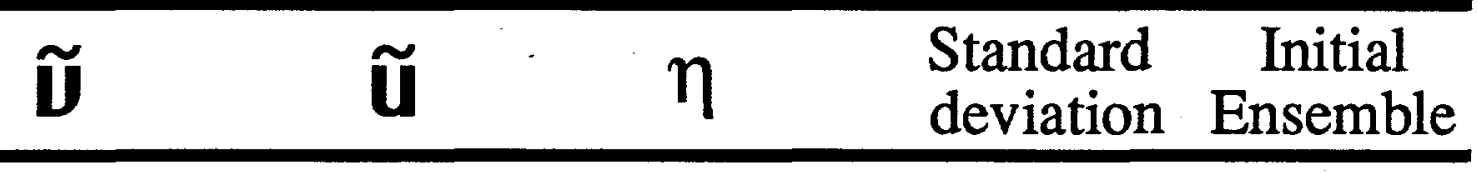

0.30

0.6012

0.0036

18942

0.32

0.5618

0.0035

19792

0.34

0.4840

0.36

0.4009

0.0035

19950

0.0036

18960

0.38

0.2669

0.0036

14716

0.40

0.1347

0.42

0.0431

0.0034

10367

0.0015

19500

0.44

0.0070

0.0006

20000

$-0.10$

$-0$.

$-0.30$

0.0000

0.0000

10000

$-0.15$

0.1857

0.0028

19352

$-0.10$

0.4680

0.0036

18996

$-0.05$

0.5903

0.0035

19488

0.00

0.6573

0.0038

16000

0.05

0.7099

0.0035

16472

0.10

0.7420

0.0036

15119

0.15

0.7532

0.0038

12655

0.28

0.7260

0.0035

15950

0.30

0.7183

0.0034

17408

0.32

0.6748

0.0033

20000

0.34

0.6051

0.0036

18648

0.36

0.4884

0.0035

19923

0.38

0.3322

0.0035

18053

0.40

0.1632

0.0035

11393

0.42

0.0533

0.44

0.0070

0.0016

19000

0.0006

20000

$-0.05$

$-0.30$

0.0002

0.0001

20000

Page: 21 
Momentum $(\mathrm{GeV})$

0.085

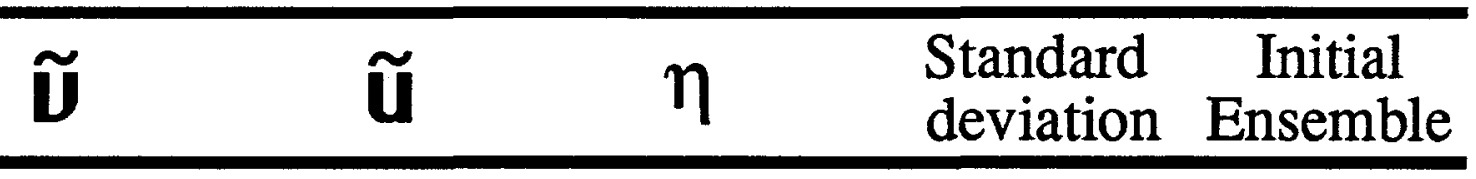

$-0.15$

0.2624

0.0035

0.0035

0.0036

0.0036

0.0034

0.0036

0.0035

0.0037

0.0038

0.0034

0.0035

0.0035

0.0035

0.0036

0.0036

0.0013

0.0323
15620

19538

16800

14254

13994

11182

11288

9804

9861

12848

13502

17715

19999

9747

19700
17081

0.00

-0.30
-0.15
-0.10
-0.05
0.00
0.05
0.10
0.15
0.28
0.30
0.32

0.0003

0.0002

10000

0.0032

18500

0.0035

19840

0.0036

17822

0.0037

16000

0.0036

14211

0.0035

13179

0.0041

13000

0.6839

0.0035

11705

0.0031

15500

0.0029

18000

Page: 22 
Momentum $(\mathrm{GeV})$

0.085
Standard Initial deviation Ensemble

$\begin{array}{llll}0.34 & 0.7943 & 0.0035 & 13411 \\ 0.36 & 0.7099 & 0.0035 & 16483 \\ 0.38 & 0.5451 & 0.0035 & 19878 \\ 0.40 & 0.3646 & 0.0036 & 17742 \\ 0.42 & 0.1867 & 0.0036 & 11808 \\ 0.44 & 0.0476 & 0.0015 & 19000 \\ 0.45 & 0.0143 & 0.0008 & 20000\end{array}$

0.05

$\begin{array}{rrrr}-0.30 & 0.0001 & 0.0000 & 20000 \\ -0.15 & 0.2589 & 0.0034 & 16233 \\ -0.10 & 0.5434 & 0.0035 & 19994 \\ -0.05 & 0.6774 & 0.0035 & 17350 \\ 0.00 & 0.7316 & 0.0035 & 15841 \\ 0.05 & 0.7880 & 0.0035 & 13683 \\ 0.10 & 0.8133 & 0.0035 & 12411 \\ 0.15 & 0.8285 & 0.0036 & 10806 \\ 0.28 & 0.8339 & 0.0036 & 10588 \\ 0.30 & 0.8264 & 0.0036 & 11288 \\ 0.32 & 0.8002 & 0.0036 & 12460 \\ 0.34 & 0.7691 & 0.0034 & 15237 \\ 0.36 & 0.6486 & 0.0035 & 18271 \\ 0.38 & 0.4781 & 0.0035 & 19831 \\ 0.40 & 0.2857 & 0.0036 & 16128 \\ 0.42 & 0.1248 & 0.0036 & 8204 \\ 0.44 & 0.0285 & 0.0012 & 19800\end{array}$

0.10

$\begin{array}{llll}-0.30 & 0.0002 & 0.0001 & 10000 \\ -0.15 & 0.1555 & 0.0027 & 18200\end{array}$

Page: 23 
Momentum $(\mathrm{GeV})$

0.085 $\eta$

$-0.10$

$-0.05$

0.00

0.05

0.10

0.15

0.28

0.30

0.32

0.34

0.36

0.38

0.40

0.42

0.44

0.11

$-0.30$

$-0.15$

$-0.10$

$-0.05$

0.00

0.05

0.10

0.15

0.28

0.30

0.32

0.34
0.4082

0.5182

0.5995

0.6488

0.6843

0.7077

0.6803

0.6743

0.6366

0.5498

0.4393

0.2978

0.1518

0.0461

0.0075
Standard deviation Ensemble 
Momentum $(\mathrm{GeV})$

0.085 $\eta$

0.3373

0.2274

0.1127

0.0349

0.0048
Standard deviation Ensemble

0.12

$\begin{array}{llll}-0.30 & 0.0002 & 0.0001 & 10000 \\ -0.15 & 0.0592 & 0.0021 & 12558 \\ -0.10 & 0.1656 & 0.0035 & 11498 \\ -0.05 & 0.2262 & 0.0036 & 13548 \\ 0.00 & 0.2833 & 0.0036 & 16000 \\ 0.05 & 0.3278 & 0.0035 & 17742 \\ 0.10 & 0.3525 & 0.0035 & 18960 \\ 0.15 & 0.3721 & 0.0034 & 19775 \\ 0.28 & 0.3607 & 0.0036 & 18295 \\ 0.30 & 0.3515 & 0.0035 & 19118 \\ 0.32 & 0.3251 & 0.0034 & 18710 \\ 0.34 & 0.2784 & 0.0036 & 15544 \\ 0.36 & 0.2188 & 0.0035 & 13638 \\ 0.38 & 0.1512 & 0.0035 & 10312 \\ 0.40 & 0.0684 & 0.0021 & 15000 \\ 0.42 & 0.0223 & 0.0010 & 20000 \\ 0.44 & 0.0033 & 0.0004 & 20000\end{array}$

Page: 25 
Momentum $(\mathrm{GeV})$

0.100 $\eta$

Standard Initial deviation Ensemble

$-0.12$

$\begin{array}{rrrr}-0.30 & 0.0002 & 0.0001 & 10000 \\ -0.28 & 0.0003 & 0.0001 & 20000 \\ -0.15 & 0.3004 & 0.0042 & 12162 \\ -0.10 & 0.3876 & 0.0036 & 18659 \\ -0.05 & 0.4407 & 0.0035 & 19682 \\ 0.00 & 0.4749 & 0.0038 & 17500 \\ 0.05 & 0.5025 & 0.0035 & 19999 \\ 0.10 & 0.5130 & 0.0035 & 19992 \\ 0.15 & 0.5279 & 0.0035 & 19982 \\ 0.28 & 0.4708 & 0.0035 & 19845 \\ 0.30 & 0.4580 & 0.0036 & 19692 \\ 0.32 & 0.4475 & 0.0035 & 19702 \\ 0.34 & 0.4144 & 0.0036 & 19247 \\ 0.36 & 0.3292 & 0.0036 & 17273 \\ 0.38 & 0.2263 & 0.0035 & 14081 \\ 0.40 & 0.1082 & 0.0036 & 7264 \\ 0.42 & 0.0249 & 0.0011 & 20000 \\ 0.44 & 0.0032 & 0.0004 & 20000\end{array}$

$-0.11$

$\begin{array}{rlll}-0.30 & 0.0003 & 0.0001 & 20000 \\ -0.28 & 0.0004 & 0.0001 & 20000 \\ -0.15 & 0.4880 & 0.0035 & 19998 \\ -0.10 & 0.6031 & 0.0035 & 19232 \\ -0.05 & 0.6523 & 0.0035 & 18271 \\ 0.00 & 0.6866 & 0.0036 & 16703 \\ 0.05 & 0.7119 & 0.0035 & 16370 \\ 0.10 & 0.7186 & 0.0036 & 15506\end{array}$

Page: 26 


$\begin{array}{llll}0.15 & 0.7202 & 0.0036 & 15731 \\ 0.28 & 0.6727 & 0.0036 & 17142 \\ 0.30 & 0.6713 & 0.0036 & 17262 \\ 0.32 & 0.6596 & 0.0036 & 17796 \\ 0.34 & 0.6113 & 0.0035 & 19118 \\ 0.36 & 0.4934 & 0.0035 & 19988 \\ 0.38 & 0.3271 & 0.0035 & 17769 \\ 0.40 & 0.1595 & 0.0035 & 10968 \\ 0.42 & 0.0408 & 0.0014 & 19500 \\ 0.44 & 0.0036 & 0.0004 & 20000\end{array}$

$-0.10$

$\begin{array}{rrrr}-0.30 & 0.0004 & 0.0002 & 10000 \\ -0.28 & 0.0009 & 0.0002 & 20000 \\ -0.15 & 0.6376 & 0.0037 & 17267 \\ -0.10 & 0.7589 & 0.0034 & 16283 \\ -0.05 & 0.7935 & 0.0035 & 13364 \\ 0.00 & 0.8183 & 0.0031 & 15000 \\ 0.05 & 0.8292 & 0.0035 & 11705 \\ 0.10 & 0.8376 & 0.0036 & 10588 \\ 0.15 & 0.8360 & 0.0040 & 8750 \\ 0.28 & 0.7993 & 0.0036 & 12704 \\ 0.30 & 0.7898 & 0.0039 & 10752 \\ 0.32 & 0.7861 & 0.0034 & 14296 \\ 0.34 & 0.7286 & 0.0035 & 16336 \\ 0.36 & 0.5837 & 0.0035 & 19380 \\ 0.38 & 0.3908 & 0.0036 & 18730 \\ 0.40 & 0.1875 & 0.0034 & 12943 \\ 0.42 & 0.0477 & 0.0015 & 19500\end{array}$


Momentum $(\mathrm{GeV})$

0.100

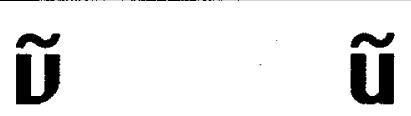

0.44 $\eta$

0.0059
Standard deviation Ensemble

$-0.05$

$\begin{array}{rrrr}-0.30 & 0.0004 & 0.0001 & 20000 \\ -0.28 & 0.0006 & 0.0002 & 20000 \\ -0.15 & 0.7639 & 0.0036 & 14125 \\ -0.10 & 0.8394 & 0.0035 & 10698 \\ -0.05 & 0.8707 & 0.0035 & 9458 \\ 0.00 & 0.8964 & 0.0036 & 7072 \\ 0.05 & 0.8921 & 0.0037 & 6943 \\ 0.10 & 0.9040 & 0.0033 & 8080 \\ 0.15 & 0.9042 & 0.0037 & 6420 \\ 0.28 & 0.8931 & 0.0034 & 8326 \\ 0.30 & 0.8953 & 0.0037 & 6942 \\ 0.32 & 0.8813 & 0.0035 & 8569 \\ 0.34 & 0.8521 & 0.0037 & 9107 \\ 0.36 & 0.7337 & 0.0037 & 13994 \\ 0.38 & 0.5432 & 0.0035 & 19878 \\ 0.40 & 0.3365 & 0.0035 & 18271 \\ 0.42 & 0.1501 & 0.0036 & 10031 \\ 0.44 & 0.0280 & 0.0012 & 19800\end{array}$

0.00

$\begin{array}{rlll}-0.32 & 0.0004 & 0.0001 & 16500 \\ -0.30 & 0.0005 & 0.0002 & 10000 \\ -0.28 & 0.0011 & 0.0002 & 20000 \\ -0.15 & 0.7307 & 0.0033 & 18000 \\ -0.10 & 0.7753 & 0.0035 & 14390 \\ -0.05 & 0.7989 & 0.0035 & 12752 \\ 0.00 & 0.8519 & 0.0029 & 15500\end{array}$

Page: 28 
Momentum $(\mathrm{GeV})$

0.100 $\eta$

Standard deviation Ensemble

$\begin{array}{rrrr}0.05 & 0.8386 & 0.0033 & 12312 \\ 0.10 & 0.8602 & 0.0036 & 9342 \\ 0.15 & 0.8771 & 0.0034 & 9500 \\ 0.28 & 0.8581 & 0.0034 & 10256 \\ 0.30 & 0.8642 & 0.0035 & 9500 \\ 0.32 & 0.8630 & 0.0029 & 14000 \\ 0.34 & 0.8523 & 0.0035 & 10533 \\ 0.36 & 0.7458 & 0.0035 & 15159 \\ 0.38 & 0.5662 & 0.0035 & 19800 \\ 0.40 & 0.3711 & 0.0036 & 18271 \\ 0.42 & 0.1770 & 0.0035 & 12212 \\ 0.44 & 0.0314 & 0.0012 & 19800\end{array}$

0.05

$\begin{array}{rrrr}-0.30 & 0.0001 & 0.0001 & 20000 \\ -0.28 & 0.0008 & 0.0002 & 20000 \\ -0.15 & 0.7611 & 0.0036 & 14211 \\ -0.10 & 0.8414 & 0.0036 & 10311 \\ -0.05 & 0.8662 & 0.0033 & 10367 \\ 0.00 & 0.8947 & 0.0036 & 7391 \\ 0.05 & 0.8969 & 0.0033 & 8509 \\ 0.10 & 0.8935 & 0.0034 & 8204 \\ 0.15 & 0.9002 & 0.0036 & 6943 \\ 0.28 & 0.8895 & 0.0032 & 9342 \\ 0.30 & 0.8880 & 0.0035 & 8018 \\ 0.32 & 0.8717 & 0.0037 & 7956 \\ 0.34 & 0.8451 & 0.0036 & 9918 \\ 0.36 & 0.7139 & 0.0035 & 17051 \\ 0.38 & 0.5228 & 0.0035 & 19958\end{array}$

Page: 29 
Momentum $(\mathrm{GeV})$

0.100

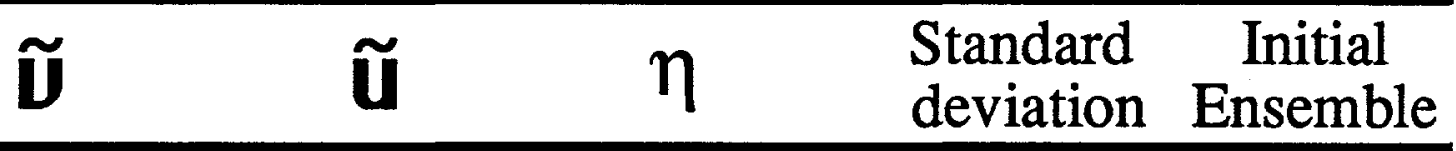

$\begin{array}{rrrr}0.40 & 0.3201 & 0.0036 & 16472 \\ 0.42 & 0.1371 & 0.0035 & 9458 \\ 0.44 & 0.0238 & 0.0011 & 19700\end{array}$

0.10

$\begin{array}{rrrr}-0.30 & 0.0002 & 0.0002 & 5000 \\ -0.28 & 0.0005 & 0.0002 & 20000 \\ -0.15 & 0.5608 & 0.0036 & 19200 \\ -0.10 & 0.6727 & 0.0035 & 18400 \\ -0.05 & 0.7220 & 0.0036 & 15768 \\ 0.00 & 0.7593 & 0.0031 & 19000 \\ 0.05 & 0.7688 & 0.0036 & 13950 \\ 0.10 & 0.7884 & 0.0036 & 13226 \\ 0.15 & 0.7881 & 0.0046 & 7818 \\ 0.28 & 0.7578 & 0.0035 & 14634 \\ 0.30 & 0.7620 & 0.0045 & 8870 \\ 0.32 & 0.7489 & 0.0044 & 9516 \\ 0.34 & 0.6907 & 0.0035 & 17232 \\ 0.36 & 0.5518 & 0.0035 & 19878 \\ 0.38 & 0.3570 & 0.0035 & 18606 \\ 0.40 & 0.1702 & 0.0035 & 11808 \\ 0.42 & 0.0432 & 0.0015 & 19500 \\ 0.44 & 0.0048 & 0.0005 & 20000\end{array}$

0.11

$\begin{array}{llll}-0.30 & 0.0002 & 0.0001 & 20000 \\ -0.28 & 0.0008 & 0.0002 & 20000 \\ -0.15 & 0.3797 & 0.0036 & 18564 \\ -0.10 & 0.4801 & 0.0035 & 19942 \\ -0.05 & 0.5272 & 0.0035 & 19946\end{array}$

Page: 30 
Momentum $(\mathrm{GeV})$

0.100 $\tilde{\mathbf{v}}$

$\tilde{\mathbf{u}}$

$\eta$

Standard Initial deviation Ensemble

0.00

0.05

0.10

0.15

0.28

0.30

0.32

0.34

0.36

0.38

0.40

0.42

0.44
0.5690

0.6022

0.6096

0.6284

0.6024

0.5981

0.5865

0.5407

0.4297

0.2938

0.1385

0.0316

0.0029
0.0050

0.0035

0.0036

0.0035

0.0036

0.0035

0.0035

0.0035

0.0035

0.0035

0.0034

0.0012

0.0004
9747

19475

18410

18905

18867

19232

19394

19890

19890

17408

10144

20000

20000

0.12

$-0.30$

$-0.28$

$-0.15$

$-0.10$

$-0.05$

0.00

0.05

0.10

0.15

0.28

0.30

0.32

0.34

0.36

0.0001

16000

0.0001

20000

0.0030

17465

0.0036

14845

0.0036

16404

0.0035

18000

0.0035

18689

0.0036

18848

0.0035

20000

0.0035

18564

0.0034

19488

0.0034

19600

0.0035

18224

0.0035

15620 


\begin{tabular}{|c|c|c|c|c|}
\hline $\begin{array}{l}\text { Momentum } \\
(\mathrm{GeV})\end{array}$ & $\tilde{\mathbf{U}}$ & $\eta$ & $\begin{array}{l}\text { Standard } \\
\text { deviation }\end{array}$ & $\begin{array}{c}\text { Initial } \\
\text { Ensemble }\end{array}$ \\
\hline \multicolumn{5}{|l|}{0.100} \\
\hline & 0.38 & 0.1757 & 0.0035 & 12162 \\
\hline & 0.40 & 0.0794 & 0.0028 & 9500 \\
\hline & 0.42 & 0.0180 & 0.0009 & 20000 \\
\hline & 0.44 & 0.0023 & 0.0003 & 20000 \\
\hline
\end{tabular}

Page: 32 
Momentum $(\mathrm{GeV})$

0.200 $\eta$

Standard Initial deviation Ensemble

$-0.12$

$\begin{array}{rrrr}-0.32 & 0.0009 & 0.0003 & 7832 \\ -0.30 & 0.0034 & 0.0006 & 8750 \\ -0.28 & 0.0525 & 0.0018 & 15040 \\ -0.15 & 0.4435 & 0.0038 & 16800 \\ -0.10 & 0.4938 & 0.0035 & 19992 \\ -0.05 & 0.5422 & 0.0035 & 19968 \\ 0.00 & 0.5724 & 0.0037 & 18000 \\ 0.05 & 0.5867 & 0.0035 & 20000 \\ 0.10 & 0.5819 & 0.0035 & 20000 \\ 0.15 & 0.5706 & 0.0035 & 20000 \\ 0.28 & 0.4411 & 0.0035 & 19775 \\ 0.30 & 0.4298 & 0.0062 & 6354 \\ 0.32 & 0.4316 & 0.0058 & 7300 \\ 0.34 & 0.4232 & 0.0035 & 19501 \\ 0.36 & 0.3541 & 0.0036 & 17550 \\ 0.38 & 0.2593 & 0.0035 & 15506 \\ 0.40 & 0.1026 & 0.0034 & 8000 \\ 0.42 & 0.0047 & 0.0005 & 20000\end{array}$

$-0.11$

$\begin{array}{rrrr}-0.32 & 0.0007 & 0.0003 & 10000 \\ -0.30 & 0.0078 & 0.0009 & 10000 \\ -0.28 & 0.1533 & 0.0037 & 9632 \\ -0.15 & 0.8023 & 0.0058 & 4715 \\ -0.10 & 0.8379 & 0.0060 & 3713 \\ -0.05 & 0.8538 & 0.0059 & 3605 \\ 0.00 & 0.8816 & 0.0053 & 3656 \\ 0.05 & 0.8910 & 0.0051 & 3671\end{array}$

Page: 33 
Momentum $(\mathrm{GeV})$

0.200 $\eta$

Standard Initial deviation Ensemble

$-0.10$

$\begin{array}{lllr}0.10 & 0.8744 & 0.0055 & 3685 \\ 0.15 & 0.8691 & 0.0053 & 4094 \\ 0.28 & 0.7976 & 0.0036 & 12162 \\ 0.30 & 0.8028 & 0.0055 & 5325 \\ 0.32 & 0.8125 & 0.0054 & 5249 \\ 0.34 & 0.8018 & 0.0036 & 12112 \\ 0.36 & 0.6494 & 0.0035 & 18176 \\ 0.38 & 0.4415 & 0.0035 & 19954 \\ 0.40 & 0.1608 & 0.0034 & 11498 \\ 0.42 & 0.0079 & 0.0006 & 20000\end{array}$

$\begin{array}{rrrr}-0.32 & 0.0007 & 0.0002 & 19032 \\ -0.30 & 0.0150 & 0.0009 & 19928 \\ -0.28 & 0.2591 & 0.0036 & 15040 \\ -0.15 & 0.9371 & 0.0017 & 19366 \\ -0.10 & 0.9512 & 0.0032 & 4512 \\ -0.05 & 0.9495 & 0.0036 & 3800 \\ 0.00 & 0.9520 & 0.0017 & 15000 \\ 0.05 & 0.9571 & 0.0033 & 3800 \\ 0.10 & 0.9530 & 0.0032 & 4512 \\ 0.15 & 0.9459 & 0.0016 & 19875 \\ 0.28 & 0.9196 & 0.0035 & 5955 \\ 0.30 & 0.9283 & 0.0018 & 19990 \\ 0.32 & 0.9294 & 0.0018 & 19662 \\ 0.34 & 0.9220 & 0.0036 & 5413 \\ 0.36 & 0.7455 & 0.0036 & 15000 \\ 0.38 & 0.4761 & 0.0035 & 19986 \\ 0.40 & 0.1547 & 0.0037 & 9690\end{array}$

Page: 34 
Momentum $(\mathrm{GeV})$

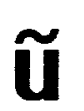

0.42 $\eta$

0.0071
Standard Initial deviation Ensemble

0.200

$-0.05$

$\begin{array}{rrrr}-0.32 & 0.0031 & 0.0004 & 19500 \\ -0.30 & 0.0647 & 0.0025 & 9500 \\ -0.28 & 0.5588 & 0.0035 & 19749 \\ -0.15 & 0.9652 & 0.0024 & 6038 \\ -0.10 & 0.9673 & 0.0024 & 5500 \\ -0.05 & 0.9696 & 0.0025 & 4800 \\ 0.00 & 0.9664 & 0.0028 & 4253 \\ 0.05 & 0.9680 & 0.0027 & 4150 \\ 0.10 & 0.9589 & 0.0031 & 4110 \\ 0.15 & 0.9666 & 0.0028 & 3983 \\ 0.28 & 0.9608 & 0.0033 & 3365 \\ 0.30 & 0.9670 & 0.0026 & 4635 \\ 0.32 & 0.9438 & 0.0032 & 5249 \\ 0.34 & 0.9512 & 0.0035 & 3872 \\ 0.36 & 0.7372 & 0.0035 & 15986 \\ 0.38 & 0.4073 & 0.0036 & 19118 \\ 0.40 & 0.0870 & 0.0030 & 9000 \\ 0.42 & 0.0055 & 0.0005 & 20000\end{array}$

0.00

-0.32
-0.30
-0.28
-0.15
-0.10
-0.05
0.00

0.0039

0.0005

19000

0.0928

0.0021

19950

0.6214

0.0036

18648

0.0024

16500

0.0028

6421

0.9478

0.0034

5888

0.0018

18000

Page: 35 
Momentum $(\mathrm{GeV})$

0.200 $\eta$

Standard Initial deviation Ensemble

$\begin{array}{rrrr}0.05 & 0.9502 & 0.0033 & 4360 \\ 0.10 & 0.9255 & 0.0040 & 4360 \\ 0.15 & 0.9490 & 0.0017 & 17500 \\ 0.28 & 0.9430 & 0.0033 & 4792 \\ 0.30 & 0.9482 & 0.0016 & 18300 \\ 0.32 & 0.9294 & 0.0019 & 18000 \\ 0.34 & 0.9278 & 0.0036 & 5139 \\ 0.36 & 0.7148 & 0.0035 & 16302 \\ 0.38 & 0.3833 & 0.0036 & 18498 \\ 0.40 & 0.0749 & 0.0025 & 11000 \\ 0.42 & 0.0060 & 0.0005 & 20000\end{array}$

0.05

$\begin{array}{rrrr}-0.32 & 0.0028 & 0.0004 & 19500 \\ -0.30 & 0.0571 & 0.0024 & 9500 \\ -0.28 & 0.5391 & 0.0035 & 19831 \\ -0.15 & 0.9626 & 0.0022 & 7672 \\ -0.10 & 0.9664 & 0.0022 & 6900 \\ -0.05 & 0.9697 & 0.0022 & 5900 \\ 0.00 & 0.9675 & 0.0025 & 5146 \\ 0.05 & 0.9687 & 0.0025 & 4950 \\ 0.10 & 0.9600 & 0.0028 & 4730 \\ 0.15 & 0.9650 & 0.0027 & 4635 \\ 0.28 & 0.9669 & 0.0036 & 2478 \\ 0.30 & 0.9653 & 0.0025 & 5482 \\ 0.32 & 0.9498 & 0.0028 & 6092 \\ 0.34 & 0.9454 & 0.0032 & 5001 \\ 0.36 & 0.7494 & 0.0034 & 16022 \\ 0.38 & 0.4172 & 0.0036 & 19278\end{array}$

Page: 36 
Momentum $(\mathrm{GeV})$

0.200 $\eta$

0.40

0.42

0.10

$-0.32$

$-0.30$

$-0.28$

$-0.15$

$-0.10$

$-0.05$

0.00

0.05

0.10

0.15

0.28

0.30

0.32

0.34

0.36

0.38

0.40

0.42

0.11
0.0852

0.0058

0.0012

0.0095

0.2029

0.8867

0.9023

0.9167

0.9265

0.9315

0.9272

0.9275

0.9047

0.9038

0.9045

0.8950

0.7264

0.4713

0.1656

0.0079
Standard Initial deviation Ensemble

0.0029

9500

0.0005

20000

0.0003

18432

0.0007

19965

0.0034

13728

0.0023

19800

0.0034

7832

0.0034

6552

0.0021

15000

0.0034

5414

0.0035

5414

0.0018

19896

0.0036

6618

0.0021

19961

0.0021

19550

0.0035

7894

0.0036

15620

0.0035

19982

0.0036

10860

0.0006

20000

0.0002

10000

0.0007

10000

0.0027

10400

0.0055

8037

0.0056

7052

0.0058

6109

Page: 37 
Momentum $(\mathrm{GeV})$

0.200 $\eta$

Standard Initial deviation Ensemble

$\begin{array}{lllr}0.00 & 0.7267 & 0.0060 & 5448 \\ 0.05 & 0.7471 & 0.0061 & 5105 \\ 0.10 & 0.7530 & 0.0059 & 5400 \\ 0.15 & 0.7580 & 0.0058 & 5380 \\ 0.28 & 0.6655 & 0.0035 & 18028 \\ 0.30 & 0.6626 & 0.0057 & 6956 \\ 0.32 & 0.6511 & 0.0057 & 6910 \\ 0.34 & 0.6586 & 0.0035 & 18318 \\ 0.36 & 0.5363 & 0.0035 & 19890 \\ 0.38 & 0.3750 & 0.0035 & 18648 \\ 0.40 & 0.1428 & 0.0036 & 9225 \\ 0.42 & 0.0070 & 0.0006 & 20000\end{array}$

\subsection{2}

$\begin{array}{rrrr}-0.32 & 0.0009 & 0.0004 & 6552 \\ -0.30 & 0.0029 & 0.0006 & 7200 \\ -0.28 & 0.0202 & 0.0010 & 19360 \\ -0.15 & 0.1993 & 0.0045 & 7894 \\ -0.10 & 0.2402 & 0.0036 & 14168 \\ -0.05 & 0.2804 & 0.0035 & 16128 \\ 0.00 & 0.3141 & 0.0048 & 9500 \\ 0.05 & 0.3323 & 0.0036 & 17350 \\ 0.10 & 0.3429 & 0.0036 & 17494 \\ 0.15 & 0.3270 & 0.0053 & 7832 \\ 0.28 & 0.2488 & 0.0035 & 15620 \\ 0.30 & 0.2294 & 0.0055 & 5888 \\ 0.32 & 0.2207 & 0.0055 & 5618 \\ 0.34 & 0.2276 & 0.0034 & 15620 \\ 0.36 & 0.1921 & 0.0035 & 12362\end{array}$

Page: 38 


\section{Momentum} $(\mathrm{GeV})$ 0.200

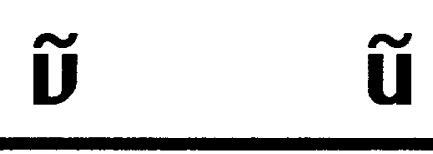

0.38

0.1416

0.40

0.0615

0.42

0.0042

Standard

Initial deviation Ensemble

0.0035

9981

0.0021

13000

0.0005 


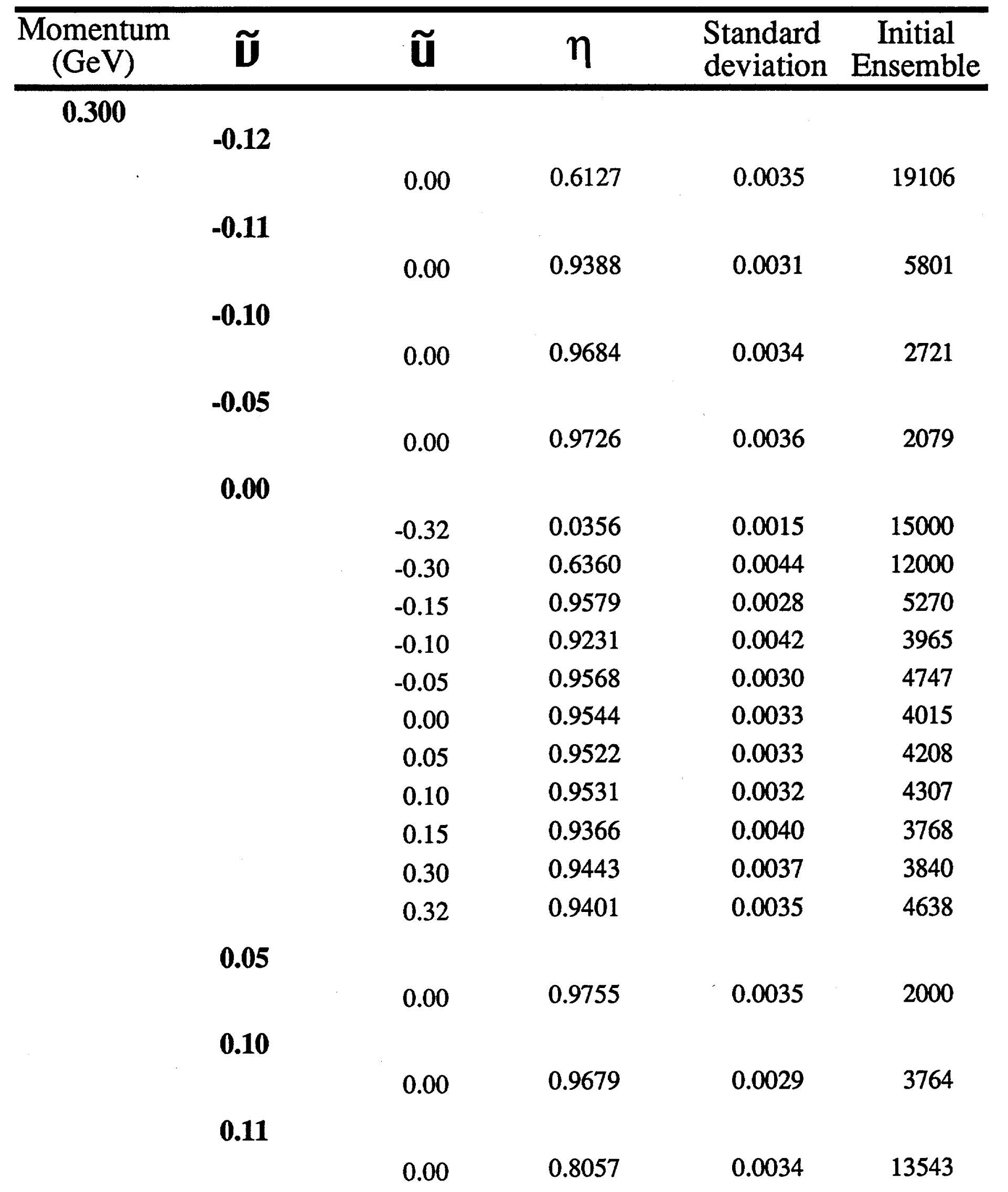




\begin{tabular}{cccccc}
\hline $\begin{array}{c}\text { Momentum } \\
(\mathrm{GeV})\end{array}$ & $\tilde{\mathbf{U}}$ & $\tilde{\mathbf{U}}$ & $\eta$ & $\begin{array}{c}\text { Standard } \\
\text { deviation }\end{array}$ & $\begin{array}{c}\text { Initial } \\
\text { Ensemble }\end{array}$ \\
\hline & $\mathbf{0 . 1 2}$ & 0.00 & 0.2783 & 0.0035 & 16415
\end{tabular}

Page: 41 
Momentum $(\mathrm{GeV})$

0.400
Standard Initial deviation Ensemble

$-0.12$

$\begin{array}{rrrr}-0.32 & 0.0056 & 0.0011 & 5000 \\ -0.30 & 0.1050 & 0.0048 & 4087 \\ -0.28 & 0.2149 & 0.0035 & 13994 \\ -0.15 & 0.4945 & 0.0036 & 19779 \\ -0.10 & 0.5566 & 0.0035 & 19854 \\ -0.05 & 0.6076 & 0.0035 & 19340 \\ 0.00 & 0.6390 & 0.0035 & 19300 \\ 0.05 & 0.6422 & 0.0035 & 18738 \\ 0.10 & 0.6361 & 0.0035 & 18993 \\ 0.15 & 0.5988 & 0.0035 & 19200 \\ 0.28 & 0.3807 & 0.0035 & 18978 \\ 0.30 & 0.3543 & 0.0034 & 19608 \\ 0.32 & 0.3340 & 0.0035 & 18200 \\ 0.34 & 0.3025 & 0.0036 & 16232 \\ 0.36 & 0.1862 & 0.0035 & 12262 \\ 0.38 & 0.0514 & 0.0022 & 10000 \\ 0.40 & 0.0034 & 0.0004 & 20000\end{array}$

$-0.11$

$\begin{array}{rrrr}-0.32 & 0.0185 & 0.0013 & 10000 \\ -0.30 & 0.5322 & 0.0041 & 14662 \\ -0.28 & 0.8143 & 0.0036 & 11446 \\ -0.15 & 0.9284 & 0.0057 & 2026 \\ -0.10 & 0.9475 & 0.0050 & 2020 \\ -0.05 & 0.9607 & 0.0043 & 2010 \\ 0.00 & 0.9610 & 0.0043 & 2000 \\ 0.05 & 0.9610 & 0.0043 & 2000 \\ 0.10 & 0.9510 & 0.0048 & 2000\end{array}$

Page: 42 
Momentum $(\mathrm{GeV})$

0.400 $\eta$

Standard Initial deviation Ensemble

$-0.10$

$\begin{array}{rrrr}0.15 & 0.9560 & 0.0046 & 2000 \\ 0.28 & 0.9107 & 0.0034 & 7200 \\ 0.30 & 0.8919 & 0.0065 & 2275 \\ 0.32 & 0.8918 & 0.0068 & 2117 \\ 0.34 & 0.8144 & 0.0036 & 11757 \\ 0.36 & 0.4463 & 0.0035 & 19872 \\ 0.38 & 0.1016 & 0.0035 & 7581 \\ 0.40 & 0.0043 & 0.0005 & 20000\end{array}$

$\begin{array}{rrrr}-0.32 & 0.0366 & 0.0022 & 7300 \\ -0.30 & 0.7583 & 0.0050 & 7200 \\ -0.28 & 0.9522 & 0.0038 & 3219 \\ -0.15 & 0.9740 & 0.0011 & 19672 \\ -0.10 & 0.9795 & 0.0032 & 2000 \\ -0.05 & 0.9825 & 0.0029 & 2000 \\ 0.00 & 0.9775 & 0.0011 & 18000 \\ 0.05 & 0.9755 & 0.0035 & 2000 \\ 0.10 & 0.9775 & 0.0033 & 2000 \\ 0.15 & 0.9746 & 0.0012 & 17112 \\ 0.28 & 0.9765 & 0.0034 & 2000 \\ 0.30 & 0.9707 & 0.0012 & 19800 \\ 0.32 & 0.9728 & 0.0012 & 18200 \\ 0.34 & 0.8645 & 0.0036 & 9107 \\ 0.36 & 0.4625 & 0.0035 & 19831 \\ 0.38 & 0.0920 & 0.0028 & 11000 \\ 0.40 & 0.0041 & 0.0005 & 20000\end{array}$

$-0.05$

$-0.34$

0.0011

0.0002

20000

Page: 43 
Momentum $(\mathrm{GeV})$ 0.400 $\eta$

Standard deviation Ensemble

$\begin{array}{rrrr}-0.32 & 0.1360 & 0.0038 & 8133 \\ -0.30 & 0.9037 & 0.0028 & 11092 \\ -0.28 & 0.9671 & 0.0035 & 2553 \\ -0.15 & 0.9813 & 0.0027 & 2560 \\ -0.10 & 0.9821 & 0.0026 & 2516 \\ -0.05 & 0.9829 & 0.0026 & 2516 \\ 0.00 & 0.9802 & 0.0028 & 2471 \\ 0.05 & 0.9780 & 0.0029 & 2591 \\ 0.10 & 0.9786 & 0.0028 & 2712 \\ 0.15 & 0.9816 & 0.0025 & 2832 \\ 0.28 & 0.9787 & 0.0030 & 2253 \\ 0.30 & 0.9775 & 0.0027 & 3020 \\ 0.32 & 0.9721 & 0.0030 & 3079 \\ 0.34 & 0.8004 & 0.0035 & 13038 \\ 0.36 & 0.4105 & 0.0036 & 19200 \\ 0.38 & 0.0557 & 0.0019 & 15000 \\ 0.40 & 0.0059 & 0.0005 & 20000\end{array}$

0.00

$\begin{array}{rrrr}-0.34 & 0.0017 & 0.0003 & 20000 \\ -0.32 & 0.1931 & 0.0067 & 3500 \\ -0.30 & 0.9091 & 0.0029 & 10000 \\ -0.28 & 0.9183 & 0.0034 & 6552 \\ -0.15 & 0.9598 & 0.0014 & 19100 \\ -0.10 & 0.9476 & 0.0040 & 3072 \\ -0.05 & 0.9473 & 0.0040 & 3072 \\ 0.00 & 0.9587 & 0.0016 & 15000 \\ 0.05 & 0.9384 & 0.0039 & 3800 \\ 0.10 & 0.9603 & 0.0032 & 3800\end{array}$

Page: 44 
Momentum $(\mathrm{GeV})$

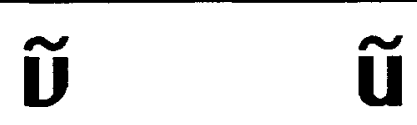

deviation Ensemble

0.400

$\begin{array}{rrrr}0.15 & 0.9519 & 0.0017 & 15000 \\ 0.28 & 0.9474 & 0.0035 & 4015 \\ 0.30 & 0.9507 & 0.0016 & 18000 \\ 0.32 & 0.9470 & 0.0017 & 17500 \\ 0.34 & 0.7711 & 0.0035 & 14037 \\ 0.36 & 0.3858 & 0.0035 & 19135 \\ 0.38 & 0.0433 & 0.0017 & 15000 \\ 0.40 & 0.0052 & 0.0005 & 20000\end{array}$

0.05

$\begin{array}{rrrr}-0.34 & 0.0013 & 0.0003 & 20000 \\ -0.32 & 0.1280 & 0.0038 & 7732 \\ -0.30 & 0.9026 & 0.0026 & 12568 \\ -0.28 & 0.9612 & 0.0034 & 3219 \\ -0.15 & 0.9727 & 0.0030 & 2858 \\ -0.10 & 0.9798 & 0.0027 & 2766 \\ -0.05 & 0.9809 & 0.0026 & 2675 \\ 0.00 & 0.9837 & 0.0025 & 2583 \\ 0.05 & 0.9795 & 0.0027 & 2687 \\ 0.10 & 0.9803 & 0.0026 & 2791 \\ 0.15 & 0.9793 & 0.0026 & 2895 \\ 0.28 & 0.9830 & 0.0029 & 2000 \\ 0.30 & 0.9800 & 0.0024 & 3292 \\ 0.32 & 0.9700 & 0.0029 & 3463 \\ 0.34 & 0.8080 & 0.0035 & 12509 \\ 0.36 & 0.4120 & 0.0035 & 19293 \\ 0.38 & 0.0533 & 0.0021 & 12000 \\ 0.40 & 0.0053 & 0.0005 & 20000\end{array}$

\subsection{0}

Page: 45 
Momentum $(\mathrm{GeV})$

0.400 $\tilde{\mathbf{v}}$

$\tilde{\mathbf{u}}$

$\eta$

Standard deviation Ensemble

$\begin{array}{rrrr}-0.32 & 0.0237 & 0.0026 & 3500 \\ -0.30 & 0.7005 & 0.0074 & 3800 \\ -0.28 & 0.9062 & 0.0035 & 6878 \\ -0.15 & 0.9660 & 0.0013 & 19992 \\ -0.10 & 0.9735 & 0.0033 & 2416 \\ -0.05 & 0.9723 & 0.0035 & 2202 \\ 0.00 & 0.9745 & 0.0012 & 17500 \\ 0.05 & 0.9702 & 0.0038 & 2049 \\ 0.10 & 0.9795 & 0.0031 & 2049 \\ 0.15 & 0.9729 & 0.0012 & 17926 \\ 0.28 & 0.9770 & 0.0034 & 2000 \\ 0.30 & 0.9633 & 0.0013 & 19950 \\ 0.32 & 0.9623 & 0.0014 & 19494 \\ 0.34 & 0.8642 & 0.0034 & 10088 \\ 0.36 & 0.4704 & 0.0035 & 19988 \\ 0.38 & 0.1022 & 0.0030 & 10000 \\ 0.40 & 0.0056 & 0.0005 & 20000\end{array}$

0.11

$\begin{array}{ll}-0.32 & 0.0101 \\ -0.30 & 0.2581 \\ -0.28 & 0.4697 \\ -0.15 & 0.7373 \\ -0.10 & 0.7815 \\ -0.05 & 0.8208 \\ 0.00 & 0.8415 \\ 0.05 & 0.8550 \\ 0.10 & 0.8454 \\ 0.15 & 0.8450\end{array}$

0.0010

10000

$0.0034 \quad 16784$

0.0035

19980

0.0086

2627

0.0091

2050

0.0085

2020

0.0082

2000

0.0078

2020

0.0080

2050

0.0079

2109

Page: 46 
Momentum $(\mathrm{GeV})$

0.400

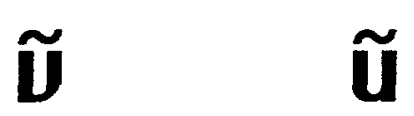

0.28

0.30

0.32

0.34

0.36

0.38

0.40 $\eta$

0.7011

0.6743

0.6533

0.5834

0.3459

0.0893

0.0041
Standard Initial deviation Ensemble

\subsection{2}

-0.32
-0.30
-0.28
-0.15
-0.10
-0.05
0.00
0.05
0.10
0.15
0.28
0.30
0.32
0.34
0.36
0.38
0.40

0.0018

0.0005

7200

0.0015

7200

0.0016

15680

0.0043

7200

0.0036

12576

0.0036

14273

0.0049

8200

0.0036

15251

0.0036

14725

0.0039

11808

0.0034

9804

0.0048

4158

0.0045

4200

0.0027

10000

0.0022

10000

0.0009

20000

0.0002

20000 
Momentum $(\mathrm{GeV})$

$\mathbf{0 . 5 0 0}$

$\eta$

0.00

0.6599

0.0035

18100

$-0.11$

0.00

0.9682

0.0035

2579

$-\mathbf{0 . 1 0}$

0.00

0.9805

0.0031

2000

$-0.05$

0.00

0.9805

0.0031

2000

0.00

$-0.32$

$-0.30$

$-0.15$

$-0.10$

$-0.05$

0.00

0.05

0.10

0.15

0.30

0.32

0.05

0.00

0.10

0.00

0.11
0.9800

0.0031

2000

0.3738

0.9338

0.9544

0.9583

0.9352

0.9608

0.9403

0.9547

0.9580

0.9486

0.9835

0.0028

2000

0.0036

18554

6092

3489

3811

4845

3138

4105

3420

3500

3543

4047

0.0034
0.0037
0.0035

4047

0.8621

0.0035

9502
0.9489 
Momentum $(\mathrm{GeV})$

-

0.00

$\tilde{\mathbf{u}}$

Standard Initial deviation Ensemble

0.12

$\eta$

0.2592

0.0035

15677 
Momentum $(\mathrm{GeV})$

0.600 $\eta$

Standard deviation Ensemble

$-0.12$

$\begin{array}{rrrr}-0.36 & 0.0002 & 0.0001 & 20000 \\ -0.34 & 0.0010 & 0.0002 & 20000 \\ -0.32 & 0.0248 & 0.0022 & 5000 \\ -0.30 & 0.1594 & 0.0056 & 4229 \\ -0.28 & 0.2348 & 0.0035 & 14340 \\ -0.15 & 0.5232 & 0.0037 & 18432 \\ -0.10 & 0.5920 & 0.0035 & 19587 \\ -0.05 & 0.6386 & 0.0035 & 18838 \\ 0.00 & 0.6692 & 0.0035 & 18500 \\ 0.05 & 0.6777 & 0.0035 & 18233 \\ 0.10 & 0.6535 & 0.0035 & 18688 \\ 0.15 & 0.6075 & 0.0035 & 19872 \\ 0.28 & 0.3591 & 0.0035 & 18790 \\ 0.30 & 0.3087 & 0.0041 & 12655 \\ 0.32 & 0.2827 & 0.0042 & 11602 \\ 0.34 & 0.2155 & 0.0035 & 13638 \\ 0.36 & 0.1080 & 0.0036 & 7500 \\ 0.38 & 0.0062 & 0.0006 & 20000\end{array}$

$-0.11$

$\begin{array}{rrrr}-0.36 & 0.0005 & 0.0002 & 20000 \\ -0.34 & 0.0008 & 0.0002 & 20000 \\ -0.32 & 0.1861 & 0.0029 & 18000 \\ -0.30 & 0.8239 & 0.0029 & 17662 \\ -0.28 & 0.8708 & 0.0035 & 9107 \\ -0.15 & 0.9639 & 0.0027 & 4903 \\ -0.10 & 0.9656 & 0.0030 & 3806 \\ -0.05 & 0.9666 & 0.0032 & 3056\end{array}$

Page: 50 
Momentum $(\mathrm{GeV})$

0.600 $\eta$

Standard Initial deviation Ensemble

$\begin{array}{lrrr}0.00 & 0.9723 & 0.0032 & 2560 \\ 0.05 & 0.9712 & 0.0032 & 2743 \\ 0.10 & 0.9746 & 0.0028 & 3233 \\ 0.15 & 0.9719 & 0.0030 & 2955 \\ 0.28 & 0.9355 & 0.0035 & 4932 \\ 0.30 & 0.9315 & 0.0030 & 6875 \\ 0.32 & 0.9149 & 0.0034 & 6919 \\ 0.34 & 0.6797 & 0.0035 & 17661 \\ 0.36 & 0.2897 & 0.0035 & 17172 \\ 0.38 & 0.0118 & 0.0008 & 20000\end{array}$

$-0.10$

$\begin{array}{rrrr}-0.36 & 0.0003 & 0.0001 & 20000 \\ -0.34 & 0.0010 & 0.0002 & 20000 \\ -0.32 & 0.3111 & 0.0038 & 15000 \\ -0.30 & 0.9560 & 0.0015 & 19758 \\ -0.28 & 0.9735 & 0.0036 & 2000 \\ -0.15 & 0.9803 & 0.0018 & 6288 \\ -0.10 & 0.9820 & 0.0030 & 2000 \\ -0.05 & 0.9820 & 0.0030 & 2000 \\ 0.00 & 0.9835 & 0.0019 & 4300 \\ 0.05 & 0.9790 & 0.0032 & 2000 \\ 0.10 & 0.9845 & 0.0028 & 2000 \\ 0.15 & 0.9803 & 0.0020 & 4862 \\ 0.28 & 0.9825 & 0.0029 & 2000 \\ 0.30 & 0.9850 & 0.0014 & 7518 \\ 0.32 & 0.9865 & 0.0026 & 2000 \\ 0.34 & 0.6987 & 0.0035 & 16736 \\ 0.36 & 0.2912 & 0.0035 & 16989\end{array}$

Page: 51 
Momentum $(\mathrm{GeV})$

0.600 $\eta$

Standard Initial deviation Ensemble
0.38
0.0119
0.0008
20000

$-0.05$

$\begin{array}{rrrr}-0.36 & 0.0007 & 0.0002 & 20000 \\ -0.34 & 0.0021 & 0.0003 & 20000 \\ -0.32 & 0.4968 & 0.0036 & 19544 \\ -0.30 & 0.9639 & 0.0028 & 4484 \\ -0.28 & 0.9750 & 0.0035 & 2000 \\ -0.15 & 0.9839 & 0.0024 & 2735 \\ -0.10 & 0.9820 & 0.0026 & 2549 \\ -0.05 & 0.9834 & 0.0021 & 3612 \\ 0.00 & 0.9828 & 0.0028 & 2215 \\ 0.05 & 0.9815 & 0.0028 & 2384 \\ 0.10 & 0.9798 & 0.0028 & 2527 \\ 0.15 & 0.9788 & 0.0029 & 2452 \\ 0.28 & 0.9825 & 0.0029 & 2000 \\ 0.30 & 0.9851 & 0.0025 & 2275 \\ 0.32 & 0.9797 & 0.0028 & 2605 \\ 0.34 & 0.6660 & 0.0035 & 17688 \\ 0.36 & 0.2524 & 0.0036 & 14508 \\ 0.38 & 0.0114 & 0.0007 & 20000\end{array}$

$\mathbf{0 . 0 0}$

$\begin{array}{rrrr}-0.36 & 0.0007 & 0.0002 & 20000 \\ -0.34 & 0.0025 & 0.0003 & 20000 \\ -0.32 & 0.5380 & 0.0079 & 4000 \\ -0.30 & 0.9248 & 0.0026 & 10000 \\ -0.28 & 0.9314 & 0.0036 & 5001 \\ -0.15 & 0.9488 & 0.0022 & 10000 \\ -0.10 & 0.9521 & 0.0036 & 3612\end{array}$

Page: 52 
Momentum $(\mathrm{GeV})$ 0.600 $\eta$

0.9232

0.9595

0.9556

0.9502

0.9564

0.9508

0.9564

0.9461

0.6425

0.2409

0.0104

0.05
0.0005

0.0019

0.4844

0.9672

0.9765

0.9847

0.9846

0.9820

0.9855

0.9833

0.9785

0.9798

0.9860

0.9817

0.9801

0.6664
0.0045

0.0033

0.0034

0.0036

0.0024

0.0038

0.0024

0.0027

0.0035

0.0035

0.0007
3580

3580

3580

3612

7000

3292

7000

7000

18564

14839

20000

20000 20000 19206

5403

2000

2802

2530

3612

2351

2512

2750

2478

2000

2672

3109

0.34

Page: 53 
Momentum $(\mathrm{GeV})$ $\tilde{\mathbf{u}}$

0.36

0.38 $\eta$

0.2491

0.0089
Standard deviation Ensemble

0.10

$\begin{array}{rrrr}-0.36 & 0.0004 & 0.0001 & 20000 \\ -0.34 & 0.0013 & 0.0002 & 20000 \\ -0.32 & 0.2627 & 0.0088 & 2478 \\ -0.30 & 0.9295 & 0.0026 & 9574 \\ -0.28 & 0.9519 & 0.0034 & 4015 \\ -0.15 & 0.9785 & 0.0010 & 19247 \\ -0.10 & 0.9825 & 0.0029 & 2000 \\ -0.05 & 0.9820 & 0.0030 & 2000 \\ 0.00 & 0.9799 & 0.0011 & 15000 \\ 0.05 & 0.9795 & 0.0032 & 2000 \\ 0.10 & 0.9785 & 0.0032 & 2000 \\ 0.15 & 0.9796 & 0.0011 & 16989 \\ 0.28 & 0.9755 & 0.0035 & 2000 \\ 0.30 & 0.9744 & 0.0011 & 19475 \\ 0.32 & 0.9729 & 0.0012 & 17688 \\ 0.34 & 0.7120 & 0.0035 & 17020 \\ 0.36 & 0.2966 & 0.0035 & 16927 \\ 0.38 & 0.0134 & 0.0008 & 20000\end{array}$

\subsection{1}

$\begin{array}{llll}-0.36 & 0.0002 & 0.0001 & 20000 \\ -0.34 & 0.0021 & 0.0003 & 20000 \\ -0.32 & 0.0693 & 0.0019 & 18000 \\ -0.30 & 0.4466 & 0.0037 & 18015 \\ -0.28 & 0.5272 & 0.0035 & 19988 \\ -0.15 & 0.7894 & 0.0033 & 15126\end{array}$

Page: 54 
Momentum $(\mathrm{GeV})$ 0.600

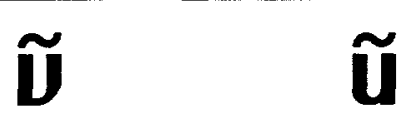

$\eta$

Standard Initial deviation Ensemble

$\begin{array}{rrrr}-0.10 & 0.8410 & 0.0031 & 13479 \\ -0.05 & 0.8648 & 0.0032 & 11341 \\ 0.00 & 0.8830 & 0.0067 & 2273 \\ 0.05 & 0.8868 & 0.0033 & 9447 \\ 0.10 & 0.8761 & 0.0032 & 10504 \\ 0.15 & 0.8588 & 0.0035 & 10154 \\ 0.28 & 0.7034 & 0.0035 & 17232 \\ 0.30 & 0.6639 & 0.0036 & 17348 \\ 0.32 & 0.6200 & 0.0036 & 17939 \\ 0.34 & 0.4795 & 0.0035 & 19896 \\ 0.36 & 0.2216 & 0.0035 & 13817 \\ 0.38 & 0.0124 & 0.0008 & 20000\end{array}$

0.12

$\begin{array}{rrrr}-0.36 & 0.0002 & 0.0001 & 20000 \\ -0.34 & 0.0011 & 0.0002 & 20000 \\ -0.32 & 0.0086 & 0.0013 & 5000 \\ -0.30 & 0.0289 & 0.0017 & 9300 \\ -0.28 & 0.0459 & 0.0016 & 17120 \\ -0.15 & 0.1573 & 0.0035 & 11030 \\ -0.10 & 0.2040 & 0.0036 & 12429 \\ -0.05 & 0.2387 & 0.0036 & 14057 \\ 0.00 & 0.2656 & 0.0037 & 14200 \\ 0.05 & 0.2622 & 0.0036 & 15008 \\ 0.10 & 0.2591 & 0.0037 & 14374 \\ 0.15 & 0.2194 & 0.0034 & 14592 \\ 0.28 & 0.1049 & 0.0036 & 7360 \\ 0.30 & 0.0864 & 0.0041 & 4792 \\ 0.32 & 0.0750 & 0.0026 & 10000\end{array}$

Page: 55 


\begin{tabular}{cccccc}
\hline $\begin{array}{c}\text { Momentum } \\
(\mathrm{GeV})\end{array}$ & $\tilde{\mathbf{U}}$ & $\tilde{\mathbf{U}}$ & $\boldsymbol{\eta}$ & $\begin{array}{c}\text { Standard } \\
\text { deviation }\end{array}$ & $\begin{array}{c}\text { Initial } \\
\text { Ensemble }\end{array}$ \\
\hline $\mathbf{0 . 6 0 0}$ & & & & \\
& 0.34 & 0.0490 & 0.0015 & 20000 \\
& 0.36 & 0.0271 & 0.0013 & 16000 \\
& 0.38 & 0.0052 & 0.0005 & 20000
\end{tabular}

Page: 56 
Momentum $(\mathrm{GeV})$

$\mathbf{0 . 8 0 0}$
Standard Initial deviation Ensemble

$-0.12$

$-0.10$

$\begin{array}{rrrr}-0.32 & 0.0458 & 0.0018 & 13000 \\ -0.30 & 0.1819 & 0.0039 & 10000 \\ -0.15 & 0.5310 & 0.0039 & 16638 \\ 0.15 & 0.6007 & 0.0035 & 19823 \\ 0.30 & 0.3103 & 0.0048 & 9342 \\ 0.32 & 0.2606 & 0.0048 & 8326\end{array}$

$\begin{array}{rrrr}-0.32 & 0.5518 & 0.0042 & 13728 \\ -0.30 & 0.9688 & 0.0023 & 5888 \\ -0.15 & 0.9774 & 0.0030 & 2478 \\ 0.15 & 0.9835 & 0.0027 & 2300 \\ 0.30 & 0.9839 & 0.0020 & 3800 \\ 0.32 & 0.3250 & 0.0057 & 6813\end{array}$

0.10

$\begin{array}{rlll}-0.32 & 0.5253 & 0.0045 & 12312 \\ -0.30 & 0.9554 & 0.0018 & 13728 \\ -0.15 & 0.9805 & 0.0010 & 18848 \\ 0.15 & 0.9826 & 0.0009 & 19032 \\ 0.30 & 0.9800 & 0.0010 & 18484 \\ 0.32 & 0.9750 & 0.0014 & 12800\end{array}$

0.12

$\begin{array}{rrrr}-0.32 & 0.0107 & 0.0011 & 9500 \\ -0.30 & 0.0407 & 0.0020 & 10000 \\ -0.15 & 0.0916 & 0.0029 & 10000 \\ 0.15 & 0.2284 & 0.0042 & 10000 \\ 0.30 & 0.0892 & 0.0029 & 10000 \\ 0.32 & 0.0748 & 0.0026 & 10000\end{array}$

Page: 57 


\begin{tabular}{cccccc}
\hline$\underset{(\mathrm{GeV})}{\operatorname{Momentum}}$ & $\tilde{\mathbf{U}}$ & $\tilde{\mathbf{U}}$ & $\eta$ & $\begin{array}{c}\text { Standard } \\
\text { deviation }\end{array}$ & $\begin{array}{c}\text { Initial } \\
\text { Ensemble }\end{array}$ \\
\hline
\end{tabular}

Page: 58 


\begin{tabular}{cccccc}
\hline $\begin{array}{c}\text { Momentum } \\
(\mathrm{GeV})\end{array}$ & $\tilde{\mathbf{U}}$ & $\tilde{\mathbf{u}}$ & $\eta$ & $\begin{array}{c}\text { Standard } \\
\text { deviation }\end{array}$ & $\begin{array}{c}\text { Initial } \\
\text { Ensemble }\end{array}$ \\
\hline
\end{tabular}

$-0.12$

$-0.10$

$\begin{array}{rrrr}-0.32 & 0.0688 & 0.0040 & 4000 \\ -0.30 & 0.2071 & 0.0068 & 3583 \\ -0.15 & 0.5511 & 0.0037 & 18432 \\ 0.00 & 0.6862 & 0.0033 & 19918 \\ 0.15 & 0.6072 & 0.0035 & 19200 \\ 0.30 & 0.3032 & 0.0048 & 9107 \\ 0.32 & 0.2429 & 0.0051 & 7040\end{array}$

$\begin{array}{rrrr}-0.32 & 0.7162 & 0.0036 & 15805 \\ -0.30 & 0.9746 & 0.0014 & 12800 \\ -0.15 & 0.9816 & 0.0027 & 2553 \\ 0.00 & 0.9832 & 0.0027 & 2328 \\ 0.15 & 0.9810 & 0.0031 & 2000 \\ 0.30 & 0.9815 & 0.0030 & 2000 \\ 0.32 & 0.9577 & 0.0037 & 3000\end{array}$

0.00

$\begin{array}{rrrr}-0.32 & 0.8560 & 0.0035 & 9918 \\ -0.30 & 0.9077 & 0.0033 & 7832 \\ -0.15 & 0.9155 & 0.0037 & 5550 \\ 0.00 & 0.9520 & 0.0029 & 5500 \\ 0.15 & 0.9440 & 0.0031 & 5500 \\ 0.30 & 0.9456 & 0.0035 & 4300 \\ 0.32 & 0.9089 & 0.0022 & 17592\end{array}$

0.10

$\begin{array}{rrrr}-0.32 & 0.6843 & 0.0047 & 9918 \\ -0.30 & 0.9658 & 0.0018 & 10000 \\ -0.15 & 0.9796 & 0.0019 & 5550\end{array}$

Page: 59 
Momentum $(\mathrm{GeV})$

1.000 $\eta$

Standard Initial deviation Ensemble

$\begin{array}{lllr}0.00 & 0.9849 & 0.0016 & 5500 \\ 0.15 & 0.9849 & 0.0016 & 5500 \\ 0.30 & 0.9865 & 0.0018 & 4300 \\ 0.32 & 0.9615 & 0.0015 & 17592\end{array}$

0.12

$\begin{array}{rrrr}-0.32 & 0.0201 & 0.0013 & 12312 \\ -0.30 & 0.0477 & 0.0018 & 13773 \\ -0.15 & 0.1901 & 0.0028 & 19500 \\ 0.00 & 0.2844 & 0.0038 & 13817 \\ 0.15 & 0.2495 & 0.0031 & 19500 \\ 0.30 & 0.1054 & 0.0023 & 18318 \\ 0.32 & 0.0864 & 0.0022 & 16500\end{array}$


Momentum $(\mathrm{GeV})$

1.300 $\eta$

Standard Initial deviation Ensemble

$-0.12$

$-0.10$

$\begin{array}{rrrr}-0.32 & 0.0873 & 0.0045 & 4000 \\ -0.30 & 0.2258 & 0.0070 & 3583 \\ -0.15 & 0.5657 & 0.0037 & 18432 \\ 0.00 & 0.6906 & 0.0033 & 19918 \\ 0.15 & 0.6124 & 0.0035 & 19200 \\ 0.30 & 0.3159 & 0.0049 & 9107 \\ 0.32 & 0.2511 & 0.0052 & 7040\end{array}$

0.00

$\begin{array}{rrrr}-0.32 & 0.8487 & 0.0029 & 15805 \\ -0.30 & 0.9779 & 0.0013 & 12800 \\ -0.15 & 0.9828 & 0.0026 & 2553 \\ 0.00 & 0.9867 & 0.0024 & 2328 \\ 0.15 & 0.9880 & 0.0024 & 2000 \\ 0.30 & 0.9855 & 0.0027 & 2000 \\ 0.32 & 0.9120 & 0.0052 & 3000\end{array}$

$\begin{array}{rrrr}-0.32 & 0.9087 & 0.0029 & 9918 \\ -0.30 & 0.9202 & 0.0031 & 7832 \\ -0.15 & 0.9092 & 0.0039 & 5550 \\ 0.00 & 0.9405 & 0.0032 & 5500 \\ 0.15 & 0.9460 & 0.0030 & 5500 \\ 0.30 & 0.9470 & 0.0034 & 4300 \\ 0.32 & 0.8699 & 0.0025 & 17592\end{array}$

0.10

$\begin{array}{llll}-0.32 & 0.8357 & 0.0037 & 9918 \\ -0.30 & 0.9667 & 0.0020 & 7832 \\ -0.15 & 0.9800 & 0.0019 & 5550\end{array}$

Page: 61 
Momentum $(\mathrm{GeV})$

1.300

$\eta$

Standard Initial deviation Ensemble

$\begin{array}{rrrr}0.00 & 0.9858 & 0.0016 & 5500 \\ 0.15 & 0.9845 & 0.0017 & 5500 \\ 0.30 & 0.9814 & 0.0021 & 4300 \\ 0.32 & 0.9226 & 0.0020 & 17592\end{array}$

\subsection{2}

$\begin{array}{llll}-0.32 & 0.0346 & 0.0016 & 12312 \\ -0.30 & 0.0690 & 0.0022 & 13773 \\ -0.15 & 0.2260 & 0.0030 & 19500 \\ 0.00 & 0.3208 & 0.0040 & 13817 \\ 0.15 & 0.2738 & 0.0032 & 19500 \\ 0.30 & 0.1276 & 0.0025 & 18318 \\ 0.32 & 0.1047 & 0.0024 & 16500\end{array}$

Page: 62 
Momentum $(\mathrm{GeV})$

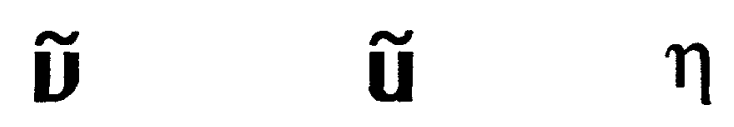

$\eta$

Standard

deviation Ensemble

\subsection{0}

$-0.12$

$-0.10$

$\begin{array}{rrrr}-0.32 & 0.1220 & 0.0073 & 2000 \\ -0.30 & 0.2507 & 0.0056 & 5888 \\ -0.15 & 0.5705 & 0.0037 & 17952 \\ 0.00 & 0.6962 & 0.0033 & 19984 \\ 0.15 & 0.6038 & 0.0035 & 19902 \\ 0.30 & 0.3216 & 0.0053 & 7832 \\ 0.32 & 0.2560 & 0.0056 & 6000\end{array}$

$\begin{array}{rrrr}-0.32 & 0.9230 & 0.0030 & 8000 \\ -0.30 & 0.9830 & 0.0017 & 6000 \\ -0.15 & 0.9810 & 0.0022 & 4000 \\ 0.00 & 0.9820 & 0.0024 & 3000 \\ 0.15 & 0.9875 & 0.0018 & 4000 \\ 0.30 & 0.9867 & 0.0015 & 6000 \\ 0.32 & 0.8863 & 0.0035 & 8000\end{array}$

0.00

$\begin{array}{rrrr}-0.32 & 0.9236 & 0.0020 & 18000 \\ -0.30 & 0.9262 & 0.0020 & 16800 \\ -0.15 & 0.9110 & 0.0040 & 5070 \\ 0.00 & 0.9307 & 0.0046 & 3000 \\ 0.15 & 0.9466 & 0.0036 & 3800 \\ 0.30 & 0.9398 & 0.0033 & 5070 \\ 0.32 & 0.8371 & 0.0027 & 18200\end{array}$

0.10

$\begin{array}{rrrr}-0.32 & 0.9051 & 0.0022 & 18000 \\ -0.30 & 0.9746 & 0.0012 & 16800 \\ -0.15 & 0.9830 & 0.0018 & 5070\end{array}$

Page: 63 
Momentum $(\mathrm{GeV})$

1.600 $\eta$

Standard Initial deviation Ensemble

$\begin{array}{rrrr}0.00 & 0.9847 & 0.0022 & 3000 \\ 0.15 & 0.9853 & 0.0020 & 3800 \\ 0.30 & 0.9824 & 0.0018 & 5070 \\ 0.32 & 0.8849 & 0.0024 & 18200\end{array}$

0.12

$\begin{array}{rlll}-0.32 & 0.0457 & 0.0019 & 12312 \\ -0.30 & 0.0906 & 0.0024 & 14675 \\ -0.15 & 0.2605 & 0.0033 & 18200 \\ 0.00 & 0.3571 & 0.0040 & 14168 \\ 0.15 & 0.3108 & 0.0035 & 17112 \\ 0.30 & 0.1619 & 0.0028 & 17292 \\ 0.32 & 0.1325 & 0.0028 & 15000\end{array}$




\section{Momentum} $(\mathrm{GeV})$

\subsection{0}

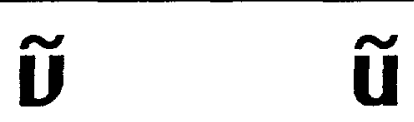

$\eta$

Standard deviation Ensemble

$-0.12$

$-0.10$

$\begin{array}{rrrr}-0.32 & 0.1422 & 0.0049 & 5000 \\ -0.30 & 0.2774 & 0.0071 & 3980 \\ -0.15 & 0.5727 & 0.0036 & 18848 \\ 0.15 & 0.6040 & 0.0035 & 19352 \\ 0.30 & 0.3293 & 0.0055 & 7263 \\ 0.32 & 0.2706 & 0.0065 & 4652\end{array}$

$\begin{array}{rrrr}-0.32 & 0.9584 & 0.0015 & 18905 \\ -0.30 & 0.9802 & 0.0012 & 13062 \\ -0.15 & 0.9835 & 0.0027 & 2177 \\ 0.15 & 0.9817 & 0.0027 & 2403 \\ 0.30 & 0.9842 & 0.0011 & 13200 \\ 0.32 & 0.8586 & 0.0025 & 19050\end{array}$

0.10

$\begin{array}{rrrr}-0.32 & 0.9494 & 0.0016 & 18867 \\ -0.30 & 0.9753 & 0.0012 & 16128 \\ -0.15 & 0.9832 & 0.0021 & 3800 \\ 0.15 & 0.9855 & 0.0019 & 3800 \\ 0.30 & 0.9854 & 0.0018 & 4466 \\ 0.32 & 0.8649 & 0.0026 & 17112\end{array}$

0.12

$\begin{array}{rlll}-0.32 & 0.0727 & 0.0023 & 12312 \\ -0.30 & 0.1259 & 0.0028 & 14467 \\ -0.15 & 0.2982 & 0.0033 & 19200 \\ 0.15 & 0.3526 & 0.0034 & 19200 \\ 0.30 & 0.1928 & 0.0030 & 17112 \\ 0.32 & 0.1604 & 0.0029 & 16472\end{array}$

Page: 65 
Momentum $(\mathrm{GeV})$

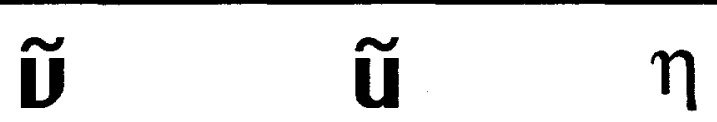

$\eta$

Standard deviation Ensemble

\subsection{0}

$-0.12$

$\begin{array}{rrrr}-0.32 & 0.1422 & 0.0049 & 5000 \\ -0.30 & 0.2774 & 0.0071 & 3980 \\ -0.15 & 0.5727 & 0.0036 & 18848 \\ 0.15 & 0.6040 & 0.0035 & 19352 \\ 0.30 & 0.3293 & 0.0055 & 7263 \\ 0.32 & 0.2706 & 0.0065 & 4652\end{array}$

$-0.10$

$\begin{array}{rrrr}-0.32 & 0.9584 & 0.0015 & 18905 \\ -0.30 & 0.9802 & 0.0012 & 13062 \\ -0.15 & 0.9835 & 0.0027 & 2177 \\ 0.15 & 0.9817 & 0.0027 & 2403 \\ 0.30 & 0.9842 & 0.0011 & 13200 \\ 0.32 & 0.8586 & 0.0025 & 19050\end{array}$

0.10

$\begin{array}{rrrr}-0.32 & 0.9494 & 0.0016 & 18867 \\ -0.30 & 0.9753 & 0.0012 & 16128 \\ -0.15 & 0.9832 & 0.0021 & 3800 \\ 0.15 & 0.9855 & 0.0019 & 3800 \\ 0.30 & 0.9854 & 0.0018 & 4466 \\ 0.32 & 0.8649 & 0.0026 & 17112\end{array}$

0.12

$\begin{array}{rlll}-0.32 & 0.0727 & 0.0023 & 12312 \\ -0.30 & 0.1259 & 0.0028 & 14467 \\ -0.15 & 0.2982 & 0.0033 & 19200 \\ 0.15 & 0.3526 & 0.0034 & 19200 \\ 0.30 & 0.1928 & 0.0030 & 17112 \\ 0.32 & 0.1604 & 0.0029 & 16472\end{array}$

Page: 65 